\title{
Modelling investigation on fiber-on-fiber contacts for synthetic fibers
}
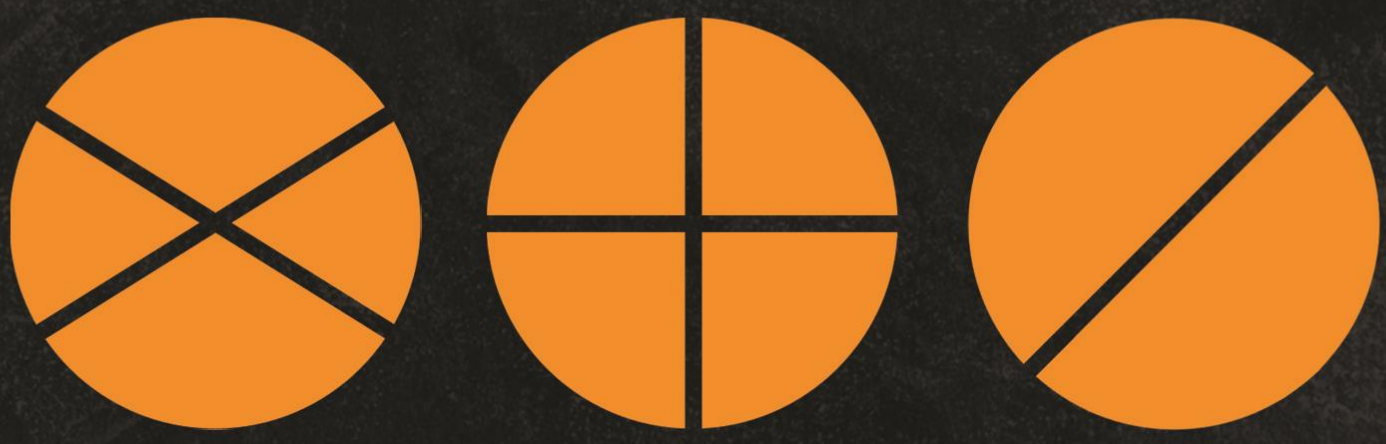

Nurul Hilwa Binti Mohd Zini 
MODELLING INVESTIGATION ON FIBER-ON-FIBER CONTACTS FOR SYNTHETIC FIBERS

Nurul Hilwa Binti Mohd Zini 



\title{
MODELLING INVESTIGATION ON FIBER-ON-FIBER CONTACTS FOR SYNTHETIC FIBERS
}

\author{
DISSERTATION \\ to obtain \\ the degree of doctor at the University of Twente, \\ on the authority of the rector magnificus, \\ prof.dr. T.T.M. Palstra, \\ on account of the decision of the Doctorate Board, \\ to be publicly defended \\ on Friday the $21^{\text {st }}$ of December 2018 at $14: 45$ hours \\ by
}

\section{Nurul Hilwa Binti Mohd Zini}

born on the $5^{\text {th }}$ of September 1985

in Johor, Malaysia 
This dissertation has been approved by:

Supervisor:

Dr.ir. M.B. de Rooij

Co-supervisor:

Prof.dr.ir. D.J. Schipper

Cover design: Mazlan Ariffin Bin Hassan

Printed by: Gildeprint

Lay-out: Nurul Hilwa Binti Mohd Zini

ISBN: 978-90-365-4636-2

DOI: $10.3990 / 1.9789036546362$

(C) 2018 Nurul Hilwa Binti Mohd Zini, The Netherlands. All rights reserved. No parts of this thesis may be reproduced, stored in a retrieval system or transmitted in any form or by any means without permission of the author. Alle rechten voorbehouden. Niets uit deze uitgave mag worden vermenigvuldigd, in enige vorm of op enige wijze, zonder voorafgaande schriftelijke toestemming van de auteur. 
GRADUATION COMMITTEE:

\section{Chairman}

Prof.dr.ir. G.P.M.R. Dewulf

University of Twente

Supervisor

Dr.ir. M.B. de Rooij

University of Twente

Co-supervisor

Prof.dr.ir. D.J. Schipper

University of Twente

Members

Prof.dr.ir. R. Akkerman

University of Twente

Prof.dr.ir. J.W.M. Noordermeer

University of Twente

Assoc. prof. dr. M.F.B. Abdollah

Universiti Teknikal Malaysia Melaka

Prof.ir.dr. J.B. A Ghani

National University of Malaysia

Dr.ir. H.J.M. Geijselaers

University of Twente 



\section{Summary}

Fiber is the main load bearer for engineering structures such as composites and rope. With characteristics such as superior mechanical strength, lightweight and flexible, synthetic fibers have replaced metals in many engineering applications. Due to its cylindrical body, contact between adjacent fibers can be subjected to various contact conditions, depending on the skew angle between the fibers. Fibers have large surface area-to-volume ratio, hence fiber contacts can be influenced by the surface forces such as adhesion. Adhesion in fiber contacts is governed by various factors such as surface roughness, intermolecular distance and the environment.

There are various contact models available that can describe adhesive contacts. However, none of these models can describe adhesive elliptical contacts that are influenced by the angle between the contacting bodies and occur between materials that are neither rigid nor highly elastic. Hence, the development of an adhesive contact model for elliptical contacts is essential.

In this thesis, the adhesive contact mechanics between fibers are investigated in the following aspects:

1. A contact model to describe the elliptical contact between cylindrical bodies with adhesion has been developed, for a range of skew angles. Realistic geometry assumptions on the load-dependent adhesive region have also been developed numerically.

2. An adhesion map for elliptical contacts has been constructed to guide the selection of suitable contact models. The construction is based on the aforementioned adhesive contact model.

3. The presence of adhesion has been shown to be significant in the contact between fibers in hierarchical structures. 



\section{Samenvatting}

De vezel is de belangrijkste lastdrager voor technische constructies zoals composieten en touw. Met eigenschappen zoals betere mechanische sterkte, lichtgewicht en flexibiliteit, hebben synthetische vezels metalen vervangen in vele technische toepassingen. Vanwege het feit dat vezels een cilindervormig lichaam hebben, kan het contact met naburige vezels worden onderworpen aan verschillende contactomstandigheden, afhankelijk van de hoek waaronder de vezels contact maken, in het vervolg hoek van scheefstand genoemd. Vezels hebben een grote oppervlakte / volumeverhouding. Adhesie, als oppervlakteverschijnsel, speelt daarom geen grote rol in het contactgedrag. Adhesie in vezelcontacten wordt in het algemeen bepaald door verschillende factoren zoals oppervlakteruwheid, intermoleculaire afstand en de omgeving.

Er zijn verschillende contactmodellen beschikbaar in de literatuur die de adhesie in dergelijke contacten kunnen beschrijven. Echter kan geen van de modellen beschrijven hoe de adhesie in de elliptische contacten wordt beïnvloed door de hoek van scheefstand tussen de vezels, waarvan de materialen noch stijf noch zeer elastisch zijn. Daarom is de ontwikkeling van een contactmodel voor elliptische contacten, inclusief de invloed van adhesie, van essentieel belang.

In dit proefschrift worden de volgende aspecten onderzocht:

1. $\mathrm{Er}$ is een model ontwikkeld dat een adhesief contact tussen twee cilindrische lichamen akn beschrijven voor verschillende hoeken van scheefstand. De geometrie van het contactgebied en de adhesieve zone zijn bepaald middels numerieke berekeningen.

2. Een adhesiemap voor elliptische contacten is geconstrueerd om de selectie van geschikte contactmodellen te begeleiden. De constructie is gebaseerd op het hiervoor genoemde adhesie-contactmodel.

3. Naast dat er aandacht besteed wordt aan contacten tussen enkele vezels, worden ook enkele hiërarchische structuren bestaande uit vezels onderzocht om te zien of adhesie inderdaad van belang is in een dergelijk contact. 



\section{Acknowledgements}

I would like to acknowledge and thank the following important people who have encouraged me to start my PhD journey, to endure with all the challenges and to finally be able to publish my thesis.

First, and most of all, I would like to express my utmost gratitude and appreciation to my supervisor and promotor, Dr. Ir. Matthijn de Rooij, for his expertise, assistance, unwavering support, guidance and patience throughout my PhD. I would also like to thank my co-promotor, Professor Dik Schipper for his valuable insight, guidance and 'travel perks'.

I want to thank the Ministry of Education Malaysia and Universiti Teknikal Malaysia Melaka for giving me the opportunity to further my study in the University of Twente.

I would like to thank the members of Surface and Tribology group at the University of Twente: Khafidh, Melkamu, Shivam, Marina, Yibo, Aydar, Mohammad, Xavier, Tanmaya, Dmitry, Dennis, Yuxin, Matthias, Michel, Belinda, Debbie, Eric, Emile, Rob, Walter and everyone else.

A special thanks to my good friend, Ida for helping me and motivating me even before I embarked my PhD journey. I will not be here without your help.

I would also like to thank all the Malaysian friends and families in Enschede: Hakim and Yana, Wan, Imrah and Nazrul, Maziah and Muaz for all those 'makan-makan' and Malaysian hospitality during all these years in Enschede. Not forgetting our Indonesian friends and also our dearest Dutch friends: Linda, Neil, Anna, Marijn, llina and Kai.

I am also grateful for the support from my family; Ibu, Ayah, Yani, Yana, Pejul and from my husband's family, especially for all those love packages all the way from Malaysia to ensure that we have an abundant supply of Malaysian food throughout our stay in the Netherlands.

Last but not least, to my dearest husband, Mazlan Ariffin Hassan and my lovely daughter, Ayuna Zara, thank you for being my ultimate pillar, following me all the way from Malaysia to ensure that I can achieve my dream. I really appreciate all the unconditional support, sacrifices and understanding from both of you when I have to put my PhD first most of the time. Both of them kept me going, and this thesis would not have been possible without them.

Nurul Hilwa Binti Mohd Zini

Enschede, the Netherlands 

Table of contents

\section{Part I}

Nomenclature i

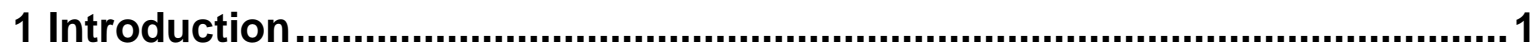

1.1 Contact mechanics modelling of fiber-on-fiber contact....................... 3

1.1.1 Non-adhesive contacts .................................................. 3

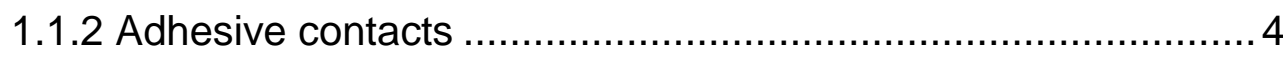

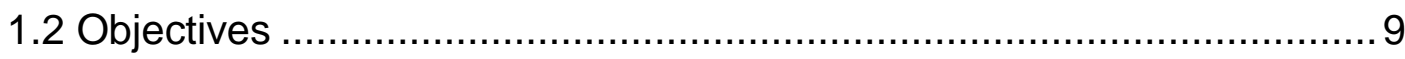

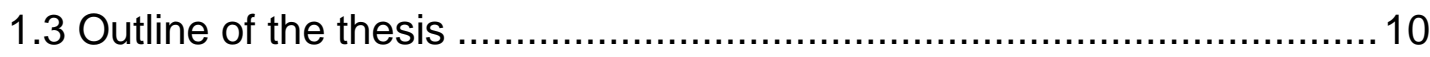

2 Adhesion modelling of nearly circular contacts .........................................13

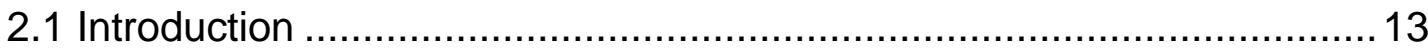

2.2 The development of a $\mathrm{DH}$ elliptical model ..................................... 14

2.3 Results and discussion ............................................................ 17

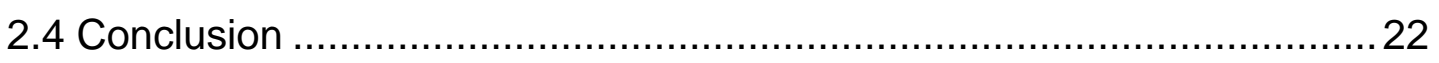

3 Adhesion modelling of elliptical contacts ................................................. 23

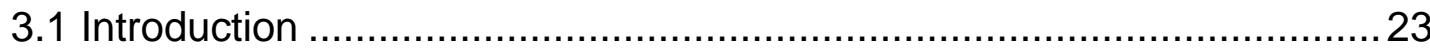

3.2 Load-dependence of $\mathrm{DH}$-based elliptical contacts ............................24

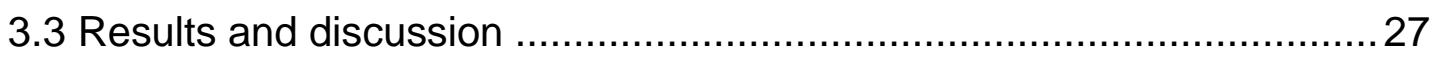

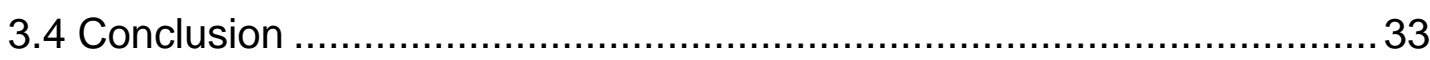

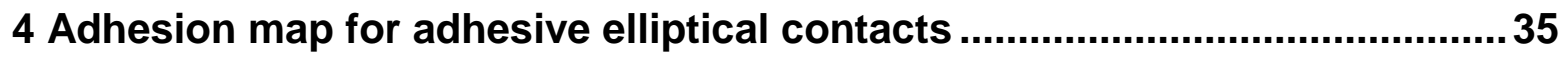

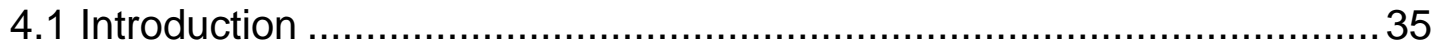

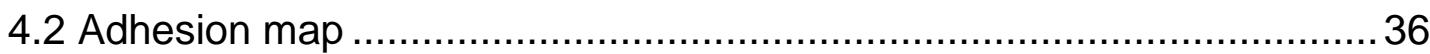

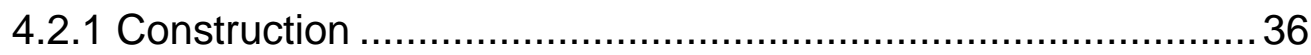

4.2.1.1 The Hertz domain ........................................ 37

4.2.1.2 The JKR domain ............................................ 37

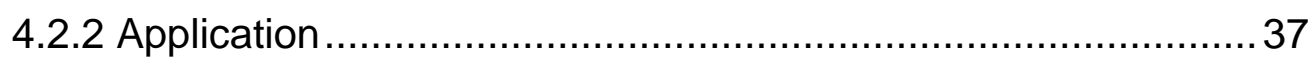

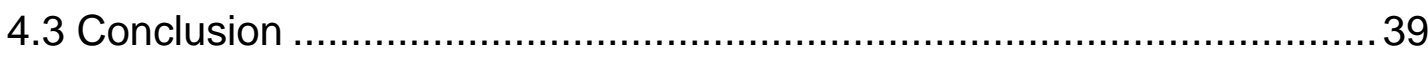


5 Adhesive nanofiber contacts in electrospun nanoyarns 41

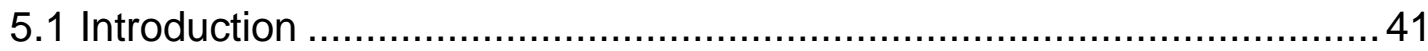

5.2 Modelling adhesive nanofiber contacts in electrospun nanoyarns .........42

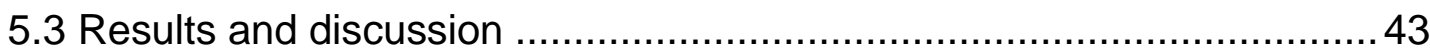

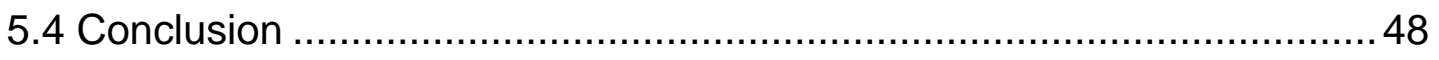

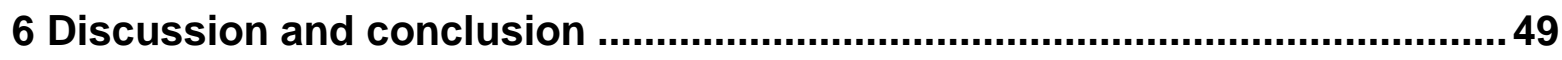

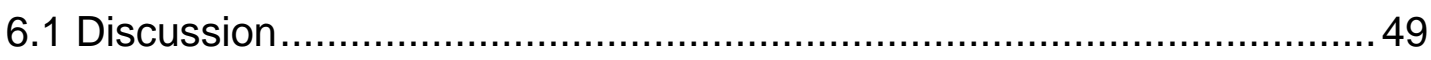

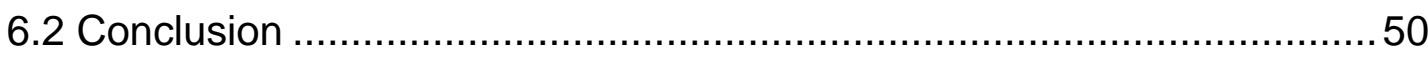

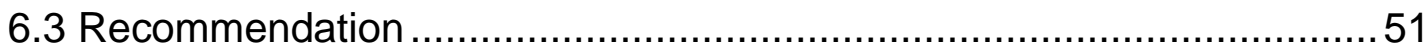

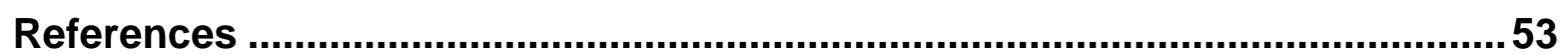




\section{Nomenclature}

*Nomenclature is divided into chapters to correlate with papers.

\section{Chapter 2}

$a, b$

$c, d$

$\beta_{0}$

$\beta_{(\text {pull-off })}$

$\beta_{J K R(p u l l-o f f)}$

$\beta$

$\beta_{a b}=b / a$

$\beta_{c d}=d / c$

$r$

$\theta_{p}$

$r_{a b}$

$r_{c d}$

$\theta_{\text {skew }}$

$E^{*}$

$E_{1}, E_{2}$

$R_{C}$

$R^{\prime}, R^{\prime \prime}$

$R=\sqrt{R^{\prime} R^{\prime \prime}}$

$\vartheta$

$\mu$

$k=\left(1-\beta^{2}\right)^{1 / 2}$

$k^{\prime}=\left(1-k^{2}\right)^{1 / 2}$

$\varphi=\sin ^{-1}\left(a / \sqrt{l+a^{2}}\right)$

$\boldsymbol{K}(k)$

$\boldsymbol{E}(k)$

$\boldsymbol{F}(\varphi, k)$

$\boldsymbol{E}(\varphi, k)$

$v$

W

$\Delta \gamma$
Semi-major and semi-minor axes of the contact ellipse

Semi-major and semi-minor axes of the adhesive ellipse

Ellipticity ratio at initial loading

Ellipticity ratio at pull-off moment

Ellipticity ratio at pull-off moment from the JKR elliptical model

Ellipticity ratio throughout contact

Ellipticity ratio of the contact ellipse

Ellipticity ratio of the adhesive ellipse

Radial coordinate

Angular coordinate

Radial coordinate of the contact ellipse

Radial coordinate of the adhesive ellipse

Angle between crossing cylinders

Reduced Young's modulus

Young moduli of the contacting materials

Radius of cylinder

Principal relative radii of curvature

Equivalent radius

Poisson's ratio

Tabor parameter

Elliptic modulus (eccentricity)

Complementary elliptic modulus

Second argument of the incomplete elliptic integrals

Complete elliptic integral of the first kind

Complete elliptic integral of the second kind

Incomplete elliptic integral of the first kind

Incomplete elliptic integral of the second kind

Scaling factor to keep $\sigma_{0}$ and $\Delta y$ at a constant value

Applied load of single asperity

Work of adhesion 


$\begin{array}{ll}P o_{a b} & \text { Maximum pressure of the contact ellipse } \\ P o_{c d} & \text { Maximum pressure of the adhesive ellipse } \\ \sigma_{0} & \text { Maximum adhesive stress } \\ \omega_{i n_{a b}} & \text { Surface displacement within the contact ellipse } \\ \omega_{o u t_{a b}} & \text { Surface displacement outside the contact ellipse } \\ \omega_{i n_{c d}} & \text { Surface displacement within the adhesive ellipse } \\ \omega_{0 a b} & \text { Combined surface displacement for } 0 \leq r \leq r_{a b} \\ \omega_{a b c d} & \text { Combined surface displacement for } r_{a b} \leq r \leq r_{c d} \\ \delta & \text { Approach of distant points } \\ z & \text { Initial gap in contact area } \\ h & \text { Separation between surfaces in the adhesive region }\end{array}$

\section{Chapter 3}

$a, b$

$c, d$

$\beta_{\text {Hertz }}$

$\beta_{0}$

$\beta_{a b}$

$\beta_{c d}$

$r$

$\theta_{p}$

$r_{a b}$

$r_{c d}$

$\theta_{\text {skew }}$

$E^{*}$

$R=\sqrt{R^{\prime} R^{\prime \prime}}$

$\mu$

$k_{a b}=\left(1-\beta_{a b}{ }^{2}\right)^{1 / 2}$

$k_{c d}=\left(1-\beta_{c d}^{2}\right)^{1 / 2}$

$k_{a b}{ }^{\prime}=\left(1-k_{a b}{ }^{2}\right)^{1 / 2}$
Semi-major and semi-minor axes of the contact ellipse at the pull-off moment

Semi-major and semi-minor axes of the adhesive ellipse at the pull-off moment

Ellipticity ratio of the Hertzian contact

Ellipticity ratio of the initial contact

Ellipticity ratio of the contact ellipse at the pull-off moment

Ellipticity ratio of the adhesive ellipse at the pull-off moment

Radial coordinate

Angular coordinate

Radial coordinate of the contact ellipse

Radial coordinate of the adhesive ellipse

Angle between two cylindrical bodies

Reduced Young's modulus

Equivalent radius

Tabor parameter

Elliptic modulus of the contact ellipse at the pull-off moment

Elliptic modulus of the adhesive ellipse at the pull-off moment

Complementary elliptic modulus of the contact ellipse at the pull-off moment 


$\begin{array}{ll}\varphi=\sin ^{-1}\left(a / \sqrt{l+a^{2}}\right) & \begin{array}{l}\text { Second argument of the incomplete elliptic integrals at the pull- } \\ \text { off moment }\end{array} \\ \boldsymbol{K}(k) & \text { Complete elliptic integral of the first kind } \\ \boldsymbol{E}(k) & \text { Complete elliptic integral of the second kind } \\ \boldsymbol{F}(\varphi, k) & \text { Incomplete elliptic integral of the first kind } \\ \boldsymbol{E}(\varphi, k) & \text { Incomplete elliptic integral of the second kind } \\ \Delta y & \text { Surface energy } \\ v & \text { Scaling factor to keep } \sigma_{0} \text { and } \Delta y \text { at a constant value } \\ W & \text { Applied load of the single asperity contact } \\ P o_{a b} & \text { Maximum pressure of the contact ellipse } \\ P o_{c d} & \text { Maximum pressure of the adhesive ellipse } \\ \omega_{i n_{a b}} & \text { Surface displacement within the contact ellipse } \\ \omega_{o u t_{a b}} & \text { Surface displacement outside the contact ellipse } \\ \omega_{i n_{c d}} & \text { Surface displacement within the adhesive ellipse } \\ \omega_{0 a b} & \text { Combined surface displacement for } 0 \leq r \leq r_{a b} \\ \omega_{a b c d} & \text { Combined surface displacement for } r_{a b} \leq r \leq r_{c d} \\ z_{0} & \text { Equilibrium separation } \\ \left.h_{0}=0.974 z_{0}\right) & \text { Separation limit between contacting surfaces for the MD model } \\ \sigma_{0}=16 \Delta y / 9 \sqrt{3} z_{0} & \text { Maximum adhesive stress for the MD model } \\ P(x, y) & \text { Applied pressure for the MD model } \\ g(x, y) & \text { Separation between two surfaces after deformation, for the MD } \\ F_{0} & \text { model } \\ & \text { Applied load for the MD model }\end{array}$

\section{Chapter 5}

$r$

D

$P$

$\alpha_{1}$

$\beta$

$R_{1}$

$\omega_{1}$

$\omega_{2}$

$n$
Nanofiber radius

SWHS structure diameter

SWHS structure pitch

Helical angle of the nanoyarn

Twist angle of a strand

Center distribution

Nanofiber twisting speed

Nanoyarn reeling speed

Number of nanofibers in a nanoyarn 
$a, b$

$\theta_{\text {skew }}$

$E^{*}$

$R^{\prime}, R^{\prime \prime}$

$R=\sqrt{R^{\prime} R^{\prime \prime}}$

$\mu$

$\Delta \gamma$

$W_{f}$

$W_{y}$

$W_{J K R}$
Semi-major and semi-minor axes of the contact ellipse at the pull-off moment

Angle between two cylindrical bodies

Reduced Young's modulus

Principal relative radii of curvature

Equivalent radius

Tabor parameter

Work of adhesion

Normal contact force between two nanofibers

Axial load of the nanoyarn

JKR Pull-off force between adjacent nanofibers 
Part I 



\section{Chapter 1}

\section{INTRODUCTION}

For many centuries, fibers have been the source of many basic human necessities including clothing, building materials and household essentials such as fishing nets. 30,000-year-old wild flax fibers were discovered in Dzudzuana Cave, Georgia, as reported in [1]. The fibers were used by the primitive people to make ropes, which were used for securing stone weapons and for weaving baskets and garments. Some of the fibers were dyed in various colours including turquoise and pink, obtained from natural sources such as roots. The discovery also found flax fibers that were weaved in a complex manner, as shown in Fig. 1.
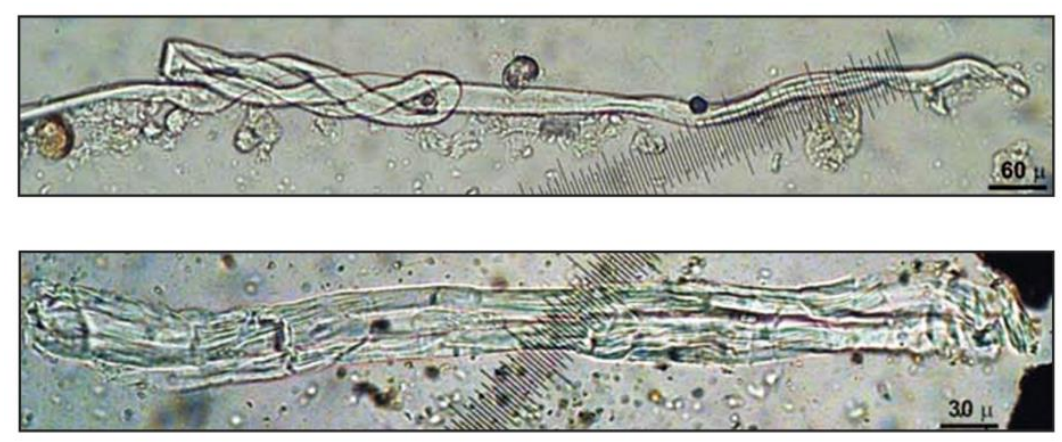

Fig. 1.1: Twisted flax fibers discovered in Dzudzuana Cave, Georgia [1].

The length of fibers is greater than their width. The hierarchical nature of the fiber structure is shown in Fig. 1.2. Fibers (microscale) are the raw material to create yarns (mesoscale); the yarns are then woven into fabrics (macroscale). Fibers can be divided into two categories; natural and synthetic fibers. Natural fibers are obtained from animals, plants and minerals, while synthetic fibers are man-made using chemical processes. 


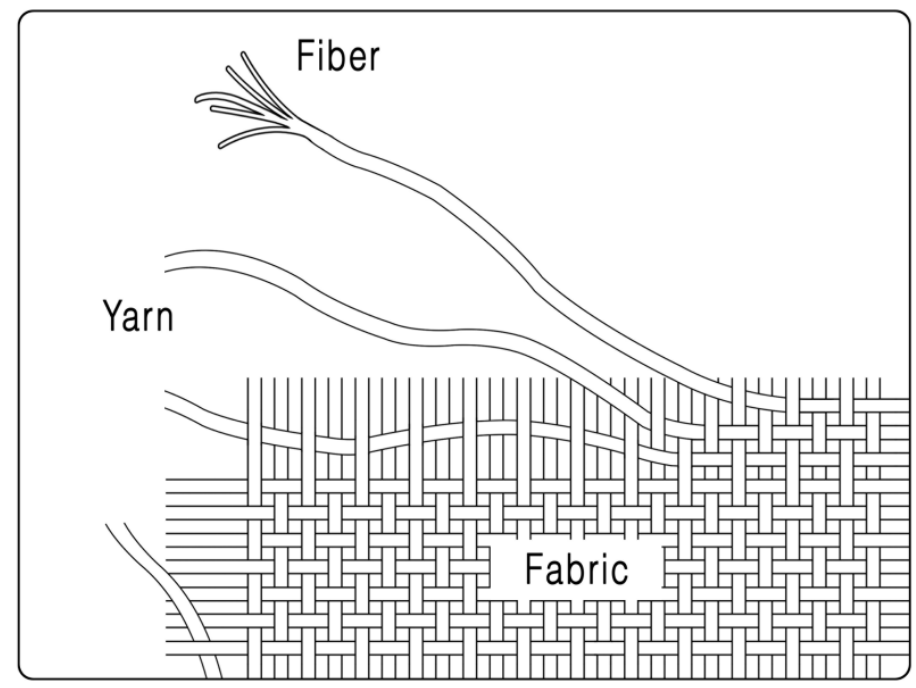

Fig. 1.2: The hierarchical nature of the fiber structure.

The demand for synthetic fibers has been increasing in recent years, in contrast to natural fibers. In 2014, the demand for synthetic fibers occupies a $67.5 \%$ share of the world fiber production, which that year recorded an annual volume of more than 96 million tonnes [2]. Synthetic fibers are more favoured than natural fibers due to the increasing demand for technical textiles with high strength and stiffness, and easier handling of the artificial fibers, amongst others. Compared with natural fibers, one of the major advantages of synthetic fibers is that they can be manufactured to cater to specific designs and industrial needs such as resistance to moisture, chemical and abrasion.

The applications of synthetic fibers are versatile, as the fiber materials can either be applied alone or combined with a wide range of other materials. Synthetic fibers are normally found in applications such as:

- Rope - Twisted fibers form the yarns which are then fabricated into strands; a group of strands will form a rope. Nylon is one of the common materials used to make ropes.

- Composites - Advanced engineering structures as the result of combining two or more materials, namely the matrix and the fibers as the reinforcement. If any of these combined materials is used independently, it will not achieve the desired properties. Fiber Reinforced Plastic (FRP) and Fiber Reinforced Concrete (FRC) are two examples of fibers that are applied in composites. 
- Fabrics - Fabric production requires textile fibers to be cut at optimum fiber length; fibers for the ring spinning process have a standard length of $4 \mathrm{~cm}$ [3]. Examples of end products are knit fabrics, laces and woven fabrics.

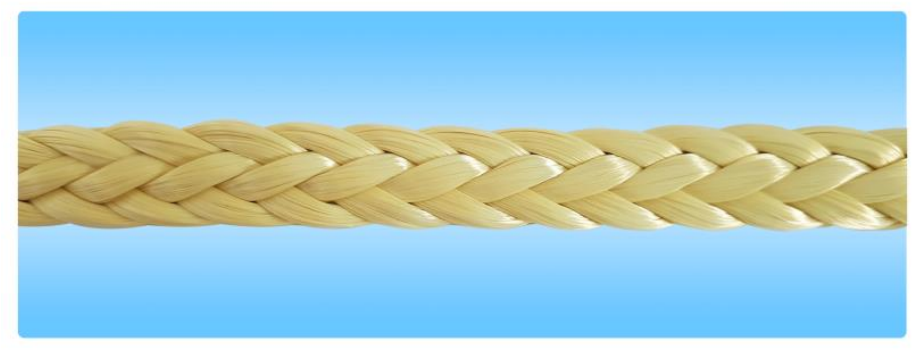

Fig. 1.3: Aramid fibers used in rope making.

There are many investigations done to develop new fibers, in a way to keep up with the increasing demands for high performance fibers. For example, a flexible, strong and sustainable material that behaves similarly as the natural spider silk was successfully developed, as reported by [4]; it composes of $98 \%$ of water, silica and cellulose. However, like other designated fibers, the mass production of the material becomes the main concern. Designated fibers can be so fine that a unique spinning system has to be designed to turn the strands into thread.

With fibers being the basic building block of many hierarchical structures as discussed previously, a deep knowledge on the contact mechanics of the fiber-on-fiber contact is a must, to ensure that the performance of the end product is not compromised. Fiber-on-fiber contact can occur in many different scales of the hierarchical structure, thus making contact mechanics modelling an important tool to study and understand the behaviour of fibers in contact. Critical parameters such as the real contact area, the interfacial separation in the non-contact regions, and the stress distribution in the contact regions can be obtained from modelling the contact between fibers.

\subsection{Contact mechanics modelling of fiber-on-fiber contacts}

\subsubsection{Non-adhesive contacts}

Contact mechanics is a study of the deformation of two solids that come into contact with one another. The classical solution by Heinrich Hertz of the contact between two elastic bodies with curved surfaces is arguably the most famous model in contact mechanics [5]. 
This non-adhesive contact problem can be generalised to other contacts, for example the contact between a rigid ball and a conforming surface, as shown in Fig. 1.4. Based on the Hertzian theory, the applied load will result in deformations, and the two surfaces will be separated as the applied load is reduced to zero. The assumptions of a Hertzian contact are (1) the contact is between smooth surfaces, (2) the contact has small deformations with no surface forces, (3) no friction influence on the contact and (4) the contact is between isotropic materials [6]. The Hertzian model is reliable for loads above a certain level but below the limit of elasticity. However, it has been shown to be incompatible for contacts with really low loads between small bodies, such as fiber-on-fiber contacts [7]. In these situations, surface forces such as adhesion can significantly affect the contact deformation.

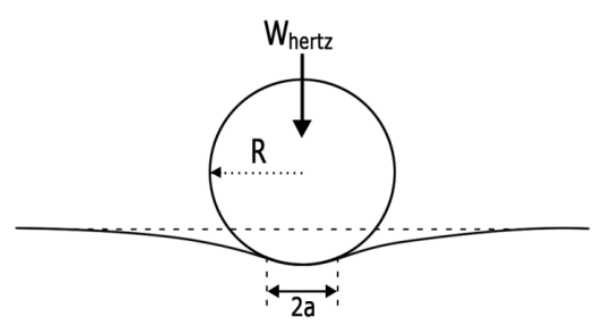

Fig. 1.4: Hertzian contact between a rigid ball and a conforming surface.

\subsubsection{Adhesive contacts}

For small-scale contacts with really low loads such as fiber-on-fiber contacts, the interactions are potentially dominated by adhesion, due to the increase of surface area-tovolume ratio as the size decreases [8]. For non-adhesive contacts, when two surfaces are loaded, the contact will be broken when the load is removed. However, for adhesive contacts, the contact between both surfaces remains at a finite value even at zero external load due to the two surfaces being pulled together by the attractive surface forces. This means that the surfaces can be separated only by a 'negative' external force, defined as the pull-off force. Due to the adhesive stresses in the non-contact area, contact deformations are larger than in non-adhesive contacts.

Currently, there are four main contact models developed for adhesive circular contacts. Using the Hertzian work as the foundation [5], the Johnson-Kendall-Roberts (JKR) model was developed for adhesive contacts [7]. Another adhesive theory was developed soon after, the Derjaguin-Muller-Toporov (DMT) model [9], the results of which contradicted the JKR solution. The disagreement between the two models was finally settled by the finding of Tabor [10], who discovered that the JKR and the DMT models are 
actually complementary. The JKR model is suitable for highly elastic materials while the DMT models are relevant for the contact of 'rigid' materials. The gap between the two models for materials that are neither rigid nor highly elastic is filled by the Maugis-Dugdale (MD) solution [11]. The Double-Hertz (DH) theory is also developed for similar contact conditions as the MD model, but one advantage it has over the MD model is that the model involves only basic mathematical formulations [12].

Each adhesive model has a specific validity domain. An adhesion map developed in [13] illustrates the applicability range for several adhesive circular models. The adhesion map, shown in Fig. 1.5, is plotted using the coordinates of the elasticity parameter $\lambda$ and the load parameter $W^{*}$. The elasticity parameter calculates the elastic deformation of the solids to the effective range of surface forces while the load parameter is the ratio of the applied load to the pull-off force.

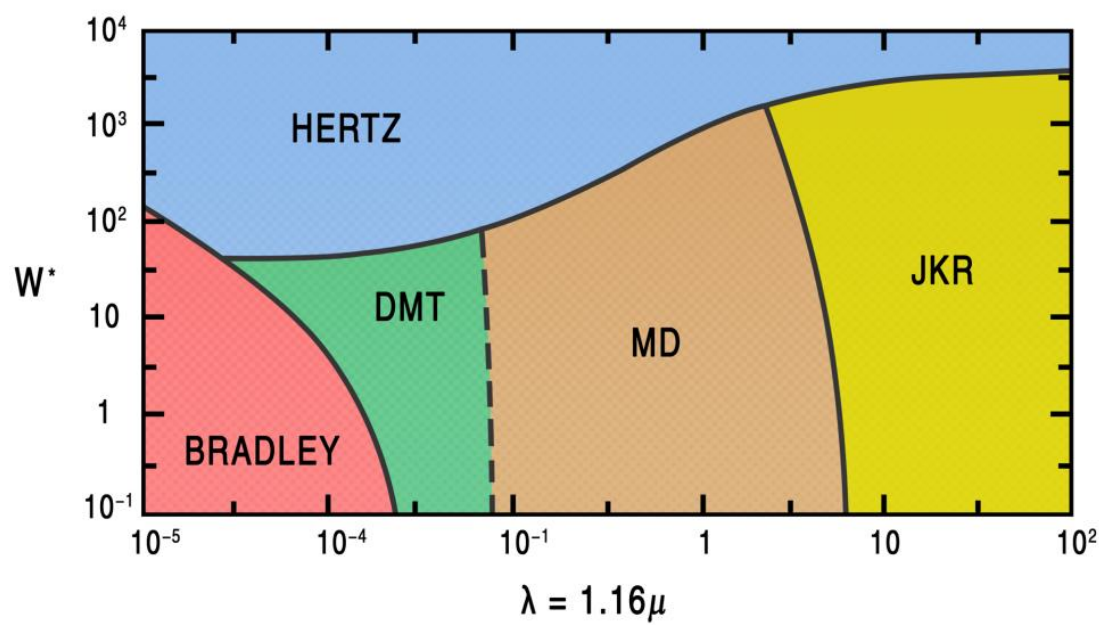

Fig. 1.5: Adhesion map for the contact of elastic spheres, from [13].

The important criterion to distinguish between the DMT and the JKR models is the neck formation outside the contact area, see Fig. 1.6. The Tabor parameter

$$
\mu=\sigma_{0}\left(R / E^{* 2} \Delta \gamma\right)^{1 / 3}
$$

is used to measure the ratio of neck height to the equilibrium separation [14]. For large values of the Tabor parameter, the adhesive stresses outside the contact area can be disregarded as the surfaces are totally separated. As the material is highly elastic, a greater neck height that corresponds to a wider gap is formed, as shown in Fig. 1.6. For this contact condition, no adhesion is present outside the contact area, which is the characteristic of a JKR contact [7]. 

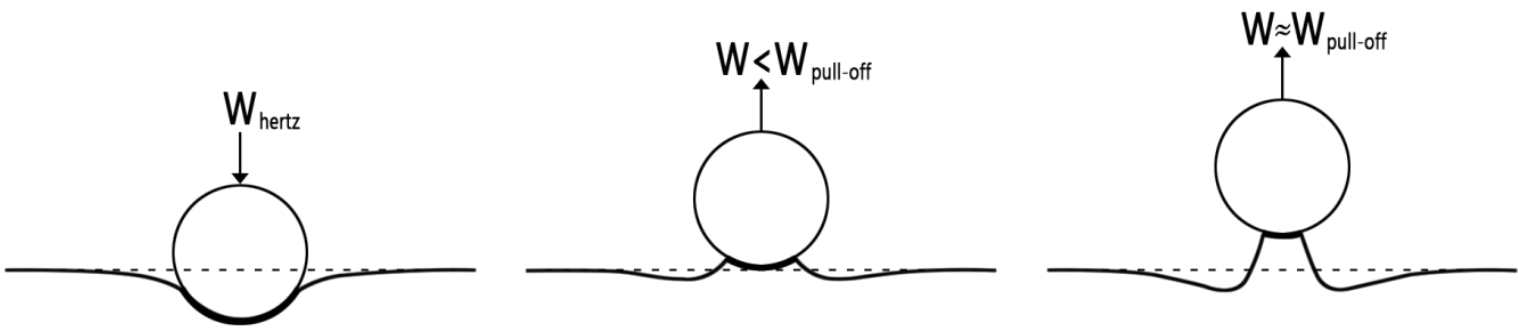

Fig. 1.6: Necking behaviour of a highly elastic surface during contact with a rigid sphere.

In the case of the DMT model [9], the presence of adhesion is significant outside the contact area as the adhesive stresses barely deform the surfaces, thus no neck is formed. The DMT contact follows a continuous and stable path until separation at a zero contact as opposed to the jumping separation for the JKR model $[6,7,14]$, which its elastic deformation in the contact area is due to the combination of contact pressure and adhesive stress [7].

The MD model occupies the intermediate zone in the adhesion map, smoothly reconciling the difference between the DMT and the JKR models. Based on the Dugdale approximation, the MD model predicts that a negative constant pressure due to adhesion acts within an annular region outside the contact area [11].

The $\mathrm{DH}$ model is a more recent addition to the adhesive contact models. The $\mathrm{DH}$ model is not part of the adhesion map in [13] but the geometrical behaviour of its annular adhesive region can be considered similar to the MD model, though the negative stress is not constant. The negative stress of the $\mathrm{DH}$ model is defined by the difference between two Hertzian pressure distributions: it has a maximum value at the inner boundary of the adhesive region and decreases to zero as it approaches the outer boundary [12]. Both the $\mathrm{MD}$ and the $\mathrm{DH}$ models cater for materials that are neither rigid nor highly elastic, thus the contact behaviour of the two models varies depending on the $\mu$ values, see Fig. 1.7. As the $\mu$ value approaches the DMT domain, the radius $a$, which is the inner boundary of the adhesive region, becomes smaller but the gap between $a$ and the radius $c$ of the outer boundary becomes larger, which is in contrast to the behaviour of materials closer to the JKR domain, where the gap becomes smaller as the a value increases. 


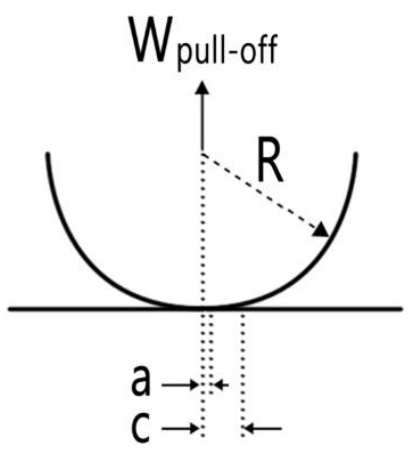

(a)

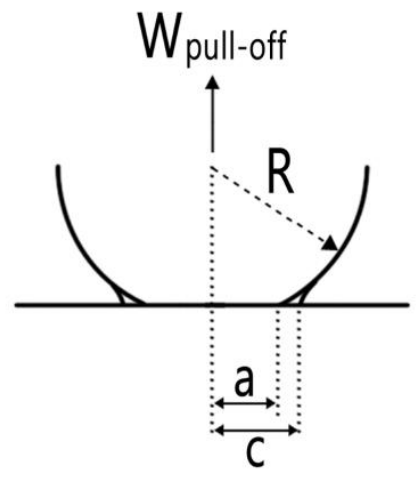

(b)

Fig. 1.7: Contact behaviour for both MD and DH model (a) for $\mu$ values approaching the DMT domain (b) for $\mu$ values approaching the JKR domain.

For fiber-on-fiber interaction, there are three possible contacts that can occur locally between the fibers: (1) the circular contact, (2) the line contact, and (3) the elliptical contact. The comparison between these contacts is shown in Table 1.1, which illustrates the effect of the skew angle between fibers on the resulting contact area. At a skew angle of $90^{\circ}$, the resulting contact area is in the shape of a circle; at an angle of $0^{\circ}$ where the fibers are parallel to each other, the resulting contact area is a line. Between the angles of $0^{\circ}$ and $90^{\circ}$, the resulting contact area is an ellipse. Fiber interactions are typically assumed to be line contacts so as to make the contact analysis simpler. However, considering that the behaviour of fiber-on-fiber contacts is not always parallel or perpendicular to each other, assuming elliptical contacts as the contact type between fibers are actually a more fitting description in many fiber contact cases. 
Table 1.1: Typical contacts between fibers.

\begin{tabular}{|l|l|}
\hline Type of fiber contact & Contact area \\
\hline $\begin{array}{l}\text { Perpendicular } \\
\left(\theta=90^{\circ}\right)\end{array}$ \\
\hline $\begin{array}{l}\text { Crossed fibers } \\
\text { with skew angle } \\
\left(0<\theta<90^{\circ}\right)\end{array}$ \\
\hline$\left(\theta=0^{\circ}\right)$
\end{tabular}

So far, only the DMT and the JKR models have been extended to all three types of fiber-on-fiber contacts, see Table 1.2. The approximate JKR model by [15] gives an insight into the behaviour of adhesive elliptical contacts for highly elastic materials. It is assumed that the contact area remains elliptical with both major and minor axes having the same values of stress intensity factor. Results show that the ellipticity ratio of the contact area varies with applied load, in contrast to the Hertzian contact behaviour where the ellipticity ratio is constant throughout the contact. When the results of the JKR model are compared with the experimental results in [16], it is shown that the two results are similar, though the errors become significant as the skew angles become lower than $20^{\circ}$. However, the model does not predict detachment, as seen in the experimental results when the skew angle approaches $0^{\circ}$. A numerical simulation by [17] is shown to have closer results to the experiments at lower skew angles. As for the DMT elliptical model [18], adhesion prediction for 'rigid' materials is based on Bradley's theory [19], while the contact geometry and the elastic deformations are obtained using the DMT approach. As the angle between the cylindrical bodies increases, the contact areas become smaller. 
Table 1.2: Comparison between existing adhesive contact models.

\begin{tabular}{|l|l|l|l|l|}
\cline { 2 - 5 } \multicolumn{1}{c|}{} & DMT & JKR & MD & DH \\
\hline $\begin{array}{l}\text { Adhesive } \\
\text { modelling }\end{array}$ & $\begin{array}{l}\text { Outside the } \\
\text { contact area }\end{array}$ & $\begin{array}{l}\text { Inside the } \\
\text { contact area }\end{array}$ & $\begin{array}{l}\text { Outside the } \\
\text { contact area }\end{array}$ & $\begin{array}{l}\text { Outside the } \\
\text { contact area }\end{array}$ \\
\hline Circular contact & $\boldsymbol{V}$ & $\boldsymbol{V}$ & $\boldsymbol{V}$ & $\boldsymbol{V}$ \\
\hline Line contact & $\boldsymbol{V}$ & $\boldsymbol{V}$ & $\boldsymbol{V}$ & \\
\hline $\begin{array}{l}\text { Elliptical } \\
\text { contact }\end{array}$ & $\boldsymbol{V}$ & $\boldsymbol{V}$ & & $\begin{array}{l}\text { Highly elastic } \\
\text { materials }\end{array}$ \\
\hline Application & $\begin{array}{l}\text { 'Rigid' } \\
\text { materials } \\
\text { elastic } \\
\text { materials }\end{array}$ & $\begin{array}{l}\text { 'Rigid' to highly } \\
\text { elastic } \\
\text { materials }\end{array}$ \\
\hline
\end{tabular}

Common synthetic fibers such as aramid are neither rigid nor highly elastic materials [20], thus are not suitable to employ the DMT and the JKR models. Considering the complexity of an elliptical contact that can vary with angles between $0^{\circ}$ to $90^{\circ}$, the best model to be extended for an adhesive elliptical contact modelling is the $\mathrm{DH}$ model as it is simpler in mathematical formulations, than the MD model [12].

\subsection{Objectives}

This study is focused on gaining insight into the microscopic interaction between synthetic fibers. The main objectives of the research are:

1. Development of a semi-analytical adhesion model for elliptical contacts between cylindrical bodies.

2. Validation of the developed model with existing adhesive contact models and experimental results.

3. Development of an adhesion map for adhesive elliptical contacts.

4. Investigation on the significance of the presence of adhesion in fiber applications.

The developed adhesive elliptical model is limited to frictionless contact between synthetic fibers, so the contact stresses are acting perpendicularly to the contacting surfaces. The developed model is based on the Double-Hertz theory, applied at skew angles between $40^{\circ}$ to $90^{\circ}$, with the intent to fill the gap of the lack of an adhesive elliptical model for fiber materials that are neither rigid nor highly elastic. This study will not determine the skew angle limit for the developed model. Multiple yarn modelling is also not 
part of this study. The nature of the selected fiber application is a simple, low order hierarchical fiber structure and consists of only fibers and yarns as its constituents.

\subsection{Outline of the thesis}

This thesis focuses on the modelling investigation of fiber-on-fiber contacts for synthetic fibers. The topic is described briefly in Chapter 1 , followed by an explanation of the contact mechanics of fiber-on-fiber interactions for both non-adhesive and adhesive contacts. The objectives of the research are also formulated in Chapter 1.

The body of the thesis is discussed in detail from Chapter 2 to Chapter 5, which are summarised from papers that are already published or submitted for publication in scientific journals. Fig. 1.8 shows how the papers are divided into the four chapters.

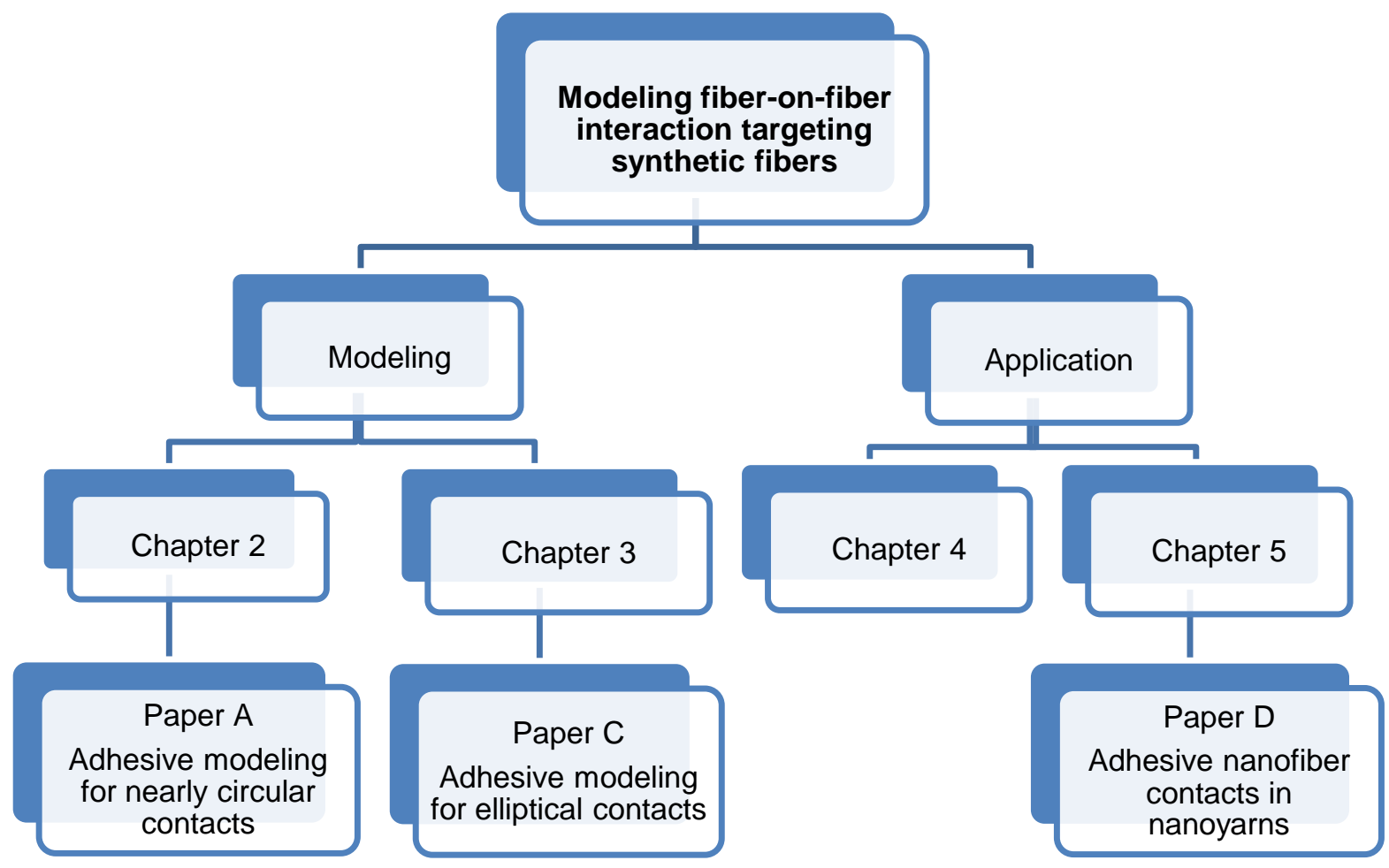

Fig. 1.8: Schematic outline of the body of the thesis.

In Chapter 2, the development of an adhesive elliptical contact model is explained in detail. Validations are done by employing the developed model for nearly circular contacts and comparing it with existing adhesive models, both circular and elliptical contacts.

In Chapter 3, the extension of the model is explained, focusing on the pull-off moment behaviour of elliptical contacts with lower skew angles. The geometry of the adhesive region at the pull-off moment is determined numerically, then the numerical data is curve-fitted to suitable equations for accurate prediction of the adhesive region at the 
pull-off moment. Similarly to the adhesion modelling of nearly circular contacts, the developed model is validated by comparing it with existing adhesive models for elliptical contacts, and also existing experimental results.

In Chapter 4, an adhesion map for adhesive elliptical contacts is constructed (in a similar way to the adhesion map in Fig. 1.5 for circular contacts) to distinguish the validity domain of existing elliptical models for single asperity contacts. Experimental results are also compared with existing adhesive models for validation purposes.

In Chapter 5, adhesive nanofiber contacts in nanoyarns are investigated. The geometrical properties during the electrospinning process are based on the model of a Stranded Wire Helical Spring (SWHS) structure, as both nanoyarns and SWHS in general have similar manufacturing process. The significance of the presence of adhesion between nanofibers in nanoyarns is determined and various parameters are tested to see their effects on adhesion between nanofibers.

In Chapter 6, the significance of adhesion in realistic fiber-on-fiber contacts is discussed. An analysis is made of whether it is important to include adhesion in the contact between fibers. The application of the developed model is also compared with the existing adhesive model. Furthermore, conclusions are also drawn from the research, together with recommendations for future modelling extensions. 



\section{Chapter 2}

\section{ADHESION MODELLING OF NEARLY CIRCULAR CONTACTS}

This chapter focuses on the development of an extended DH model for elliptical contacts and its validations for nearly circular contacts. The model validations for elliptical contacts with lower skew angles will be discussed in Chapter 3.

\subsection{Introduction}

For a circular contact, prediction of the geometry in the adhesive region is straightforward as the deformation is constant throughout the periphery, as shown in [11]. In the case of an elliptical contact, it is clearly a complex interaction, involving various contact conditions ranging from nearly circular to slim elliptical contacts. The ellipticity ratio, $\beta$ is introduced in Paper A of Part II to illustrate the deviation of the ellipse from the circular shape. For contact cases that result in an elliptical contact area characterized by semi-major axis, a and semi-minor axis, $b, \beta$ is defined as:

$$
\beta=\frac{\text { semi-minor axis }}{\text { semi-major axis }}=\frac{b}{a}
$$

From Eq. (2.1), the values of the ellipticity ratios are found to be within the range of $0<\beta<$ 1 where $\beta$ values closer to one have nearly circular contact areas, which are equivalent to having a nearly $90^{\circ}$ angle between contacting cylinders. $\beta$ values lower than one have contact areas with more elongated elliptical shape, due to small skew angles, $\theta_{\text {skew. }}$. The shape variations of an elliptical contact are shown schematically in Fig. 2.1.

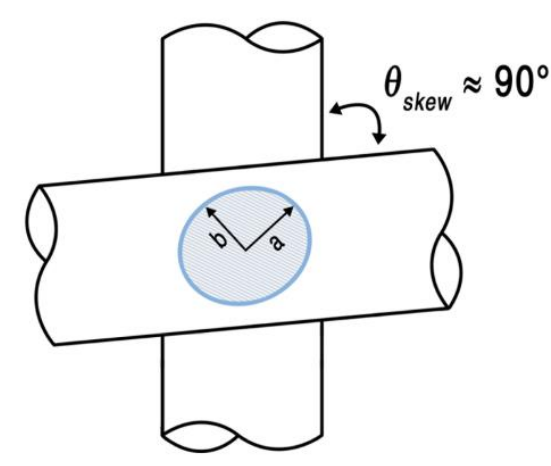

(a)

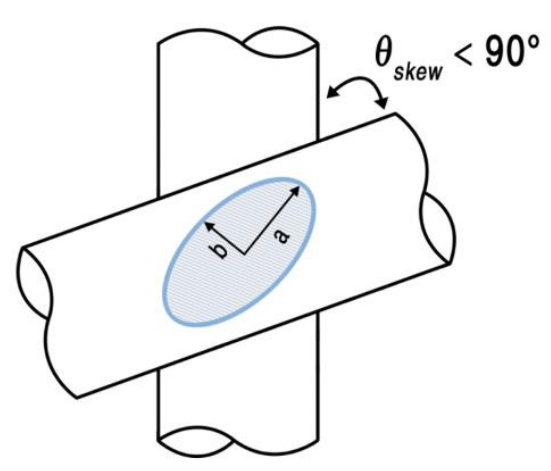

(b)

Fig. 2.1: Variation of an elliptical contact (a) Nearly circular contacts for $\beta$ value close to 1 $\left(\theta_{\text {skew }} \approx 90^{\circ}\right)$ (b) Elliptical contacts for intermediate values of $\beta\left(\theta_{\text {skew }}<90^{\circ}\right)$. 
In this chapter, the development of the $\mathrm{DH}$ model for adhesive elliptical contacts from Paper A of Part II will be explained in detail, focusing on nearly circular contacts with initial ellipticity ratios ranging from 0.8 to 0.99 . The extended $\mathrm{DH}$ model is expected to behave similarly to the current adhesive models in the limiting case of circular contacts, for materials with the Tabor parameter within the range of $0.5 \leq \mu \leq 5$. Here, both contact and adhesive ellipses which bounded the annular adhesive region are assumed to have identical, fixed ellipticity ratios throughout the contact, though the limit of this assumption must be evaluated. The extended DH model is also expected to follow the behaviour of the JKR elliptical model in the JKR domain. It is shown that the pull-off behaviour in the JKR domain is load-dependent, as shown by the unequal growth rate of its contact area in both major and minor axes directions, similar to the behaviour in [15]. However, the question of whether the adhesive region of the extended $\mathrm{DH}$ model is also subjected to the loaddependence behaviour, inside and outside the JKR domain, needs to be investigated. These aspects are also explored in this chapter.

\subsection{The development of a DH elliptical model}

From [12], the basis of the DH model for circular contacts is that the adhesive tensile stresses are represented by the difference between two Hertzian pressure distributions of contact radius, a and adhesive radius, $c$, with $a<c$, as shown in Fig. 2.2. Unlike the MD model where the adhesive tensile stress is constant within the adhesive region [11], the maximum adhesive tensile stress of the $\mathrm{DH}$ model occurs at $a$ and then decreases to approach a zero value at $c$ [12]. 


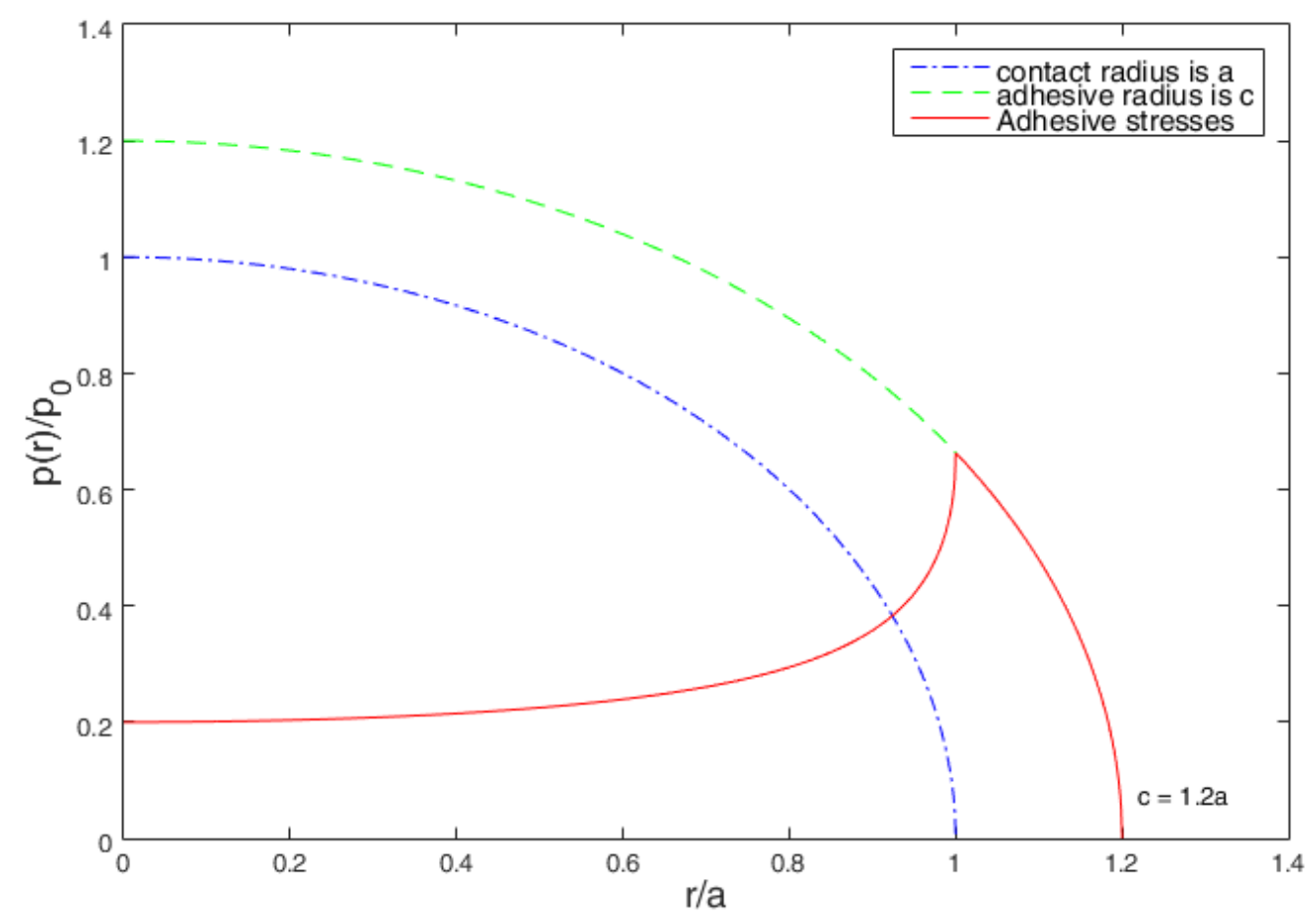

Fig. 2.2: The combination of two Hertzian pressure distributions to represent adhesive tensile stresses.

The DH theory developed in [12] has been extended to elliptical contacts in Paper A of Part II, with two Hertzian pressure distributions having equivalent shapes of elliptical contact area, the only difference being in size. The smaller ellipse acts as the inner boundary of the adhesive region, while the larger ellipse acts as the outer boundary, which both are termed as the contact and adhesive ellipses respectively, resulting in an annular region where the adhesion forces act, see Fig. 2.3. The contact ellipse is due to the applied load, and characterised by semi-major axis $a$ and semi-minor axis $b$. The additional pressure distribution outside the contact ellipse results in the adhesive ellipse, characterised by semi-major axis $c$ and semi-minor axis $d$, as shown in Fig. 2.3. The ellipticity ratio for contact and adhesive ellipses are termed $\beta_{a b}$ and $\beta_{c d}$ respectively. 


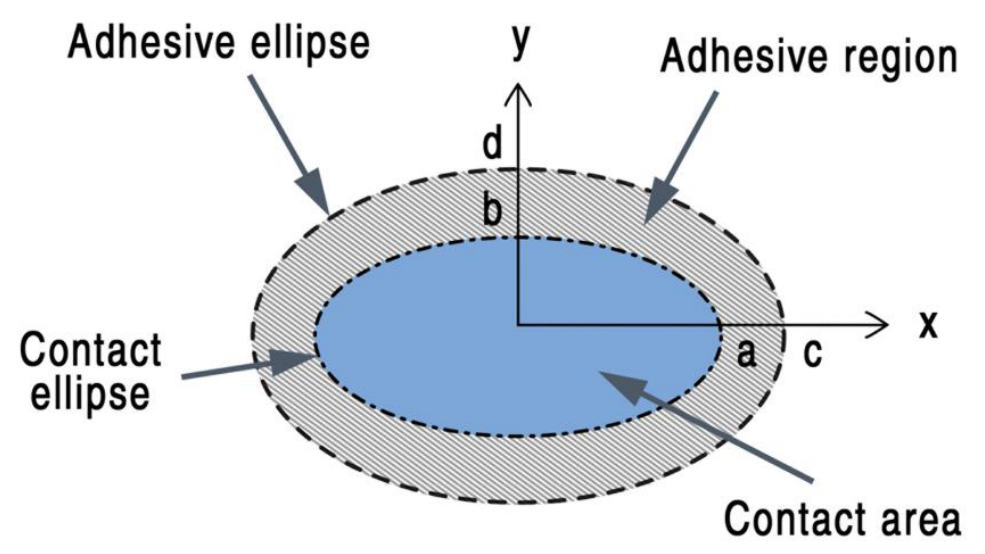

Fig. 2.3: Adhesive region for the extended DH model for elliptical contacts from Paper B of Part II.

For non-adhesive contacts, the $\beta$ value throughout the contact remains constant [21] and is equal to the ellipticity ratio at initial loading, $\beta_{0}$, which can be expressed as:

$$
\beta=\beta_{0}
$$

In the case of adhesive contacts, as considered in Paper A of Part II, it is assumed that both contact and adhesive ellipses have equal values of ellipticity ratio during contact, which is expressed as:

$$
\beta=\beta_{a b}=\beta_{c d}
$$

It is acknowledged that Eq. (2.3) is not correct for adhesive elliptical contacts; it is used as an approximation to better understand adhesive elliptical contacts, see Paper B of Part II. At the pull-off moment, the relation in Eq. (2.3) becomes:

$$
\beta_{(p u l l-o f f)}=\beta_{a b(\text { pull-off })}=\beta_{c d(p u l l-o f f)}
$$

Following the Hertzian assumption for elliptical contacts in Eq. (2.2), the relation of

$$
\beta=\beta_{(\text {pull-off })}=\beta_{0}
$$

is maintained throughout the adhesive contact. Again, similar to Eq. (2.3), it should be noted that this assumption is not correct as the ellipticity ratio clearly does not remain constant, as discussed in Paper B of Part II. The assumptions of Eq. 2.4 and Eq. 2.5 are expected to be valid for nearly circular contacts with $\beta$ values close to 1 . These assumptions are also valid for materials close to the DMT domain, as these materials are barely deformed [9]. Derivation of the extended DH model is explained in detail in Paper A of Part II. 


\subsection{Results and discussion}

The extended DH model in Paper A of Part II is validated for $\mu$ values of $0.5,1$, and 5 , with $\beta$ values of $0.8,0.9$ and 0.99 , which represent the contacts that can be considered as nearly circular contacts. Following the assumptions made in [13], $\mu=0.5$ is considered the upper limit for 'rigid' materials in the DMT domain and $\mu=5$ is the lower limit for highly elastic materials in the JKR domain. $\mu=1$ is selected to represent materials that are neither rigid nor highly elastic, as the value is between the limit of the DMT and the JKR domains. The assumptions from Eq. (2.2) to Eq. (2.5) are illustrated in Table 2.1, showing the effect of $\beta$ and material types on the adhesive regions of the extended $\mathrm{DH}$ contact. For a similar initial load, the non-adhesive contacts have the smallest contact areas; for adhesive contacts, as seen in the results of [12], materials with higher $\mu$ values have larger contact areas as highly elastic materials are easily deformed compared to 'rigid' materials. However, the adhesive region for materials with higher $\mu$ is smaller compared to materials with lower $\mu$; this is similar to the behaviour of the JKR circular contact [7] where the adhesion occurs at the edge of the contact area. As shown in Fig. 2.4, from the initial load to the pull-off moment, both contact area and adhesive region decrease in size, while maintaining the assumptions from Eq. (2.2) to Eq. (2.5). After the pull-off moment, separation of the contacting surfaces occurs at very low pull-off force values [12]. 
Table 2.1: Graphical representation of the adhesive region due to applied assumptions.
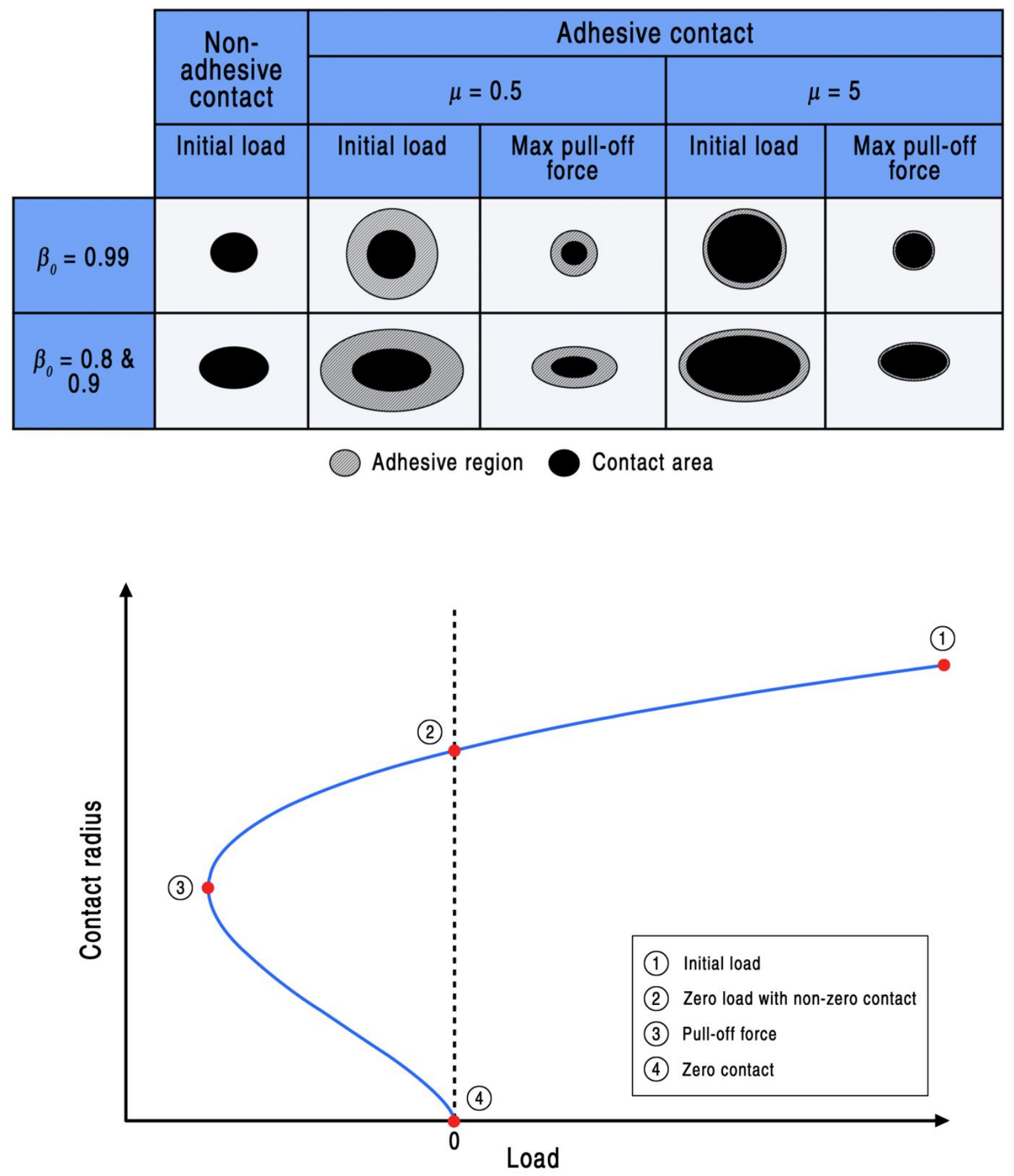

Fig. 2.4: Contact progression from initial loading to surface separation.

For $\beta_{0}=0.99$, the extended $\mathrm{DH}$ model produces results that are comparable with existing adhesive circular models, see Fig. 2.5a and Fig. 2.5b. At $\mu=1$, both the DH circular model and the extended $\mathrm{DH}$ model have similar results. The extended $\mathrm{DH}$ model at $\mu=5$ also predicts similar pull-off force as the JKR circular contacts. As $\mu$ values increase, the pull-off force becomes lower as the contacting surfaces are highly elastic and 
become easier to separate. However, for $\beta_{0}$ values of 0.8 and 0.9 , the extended DH model underestimates the pull-off force prediction, see Fig. 2.5c. As discussed in Paper A of Part II, the constant ellipticity ratio assumption in Eq. (2.5) limits the changes in the adhesive region especially in the major axis direction, hence the pull-off forces are underestimated.

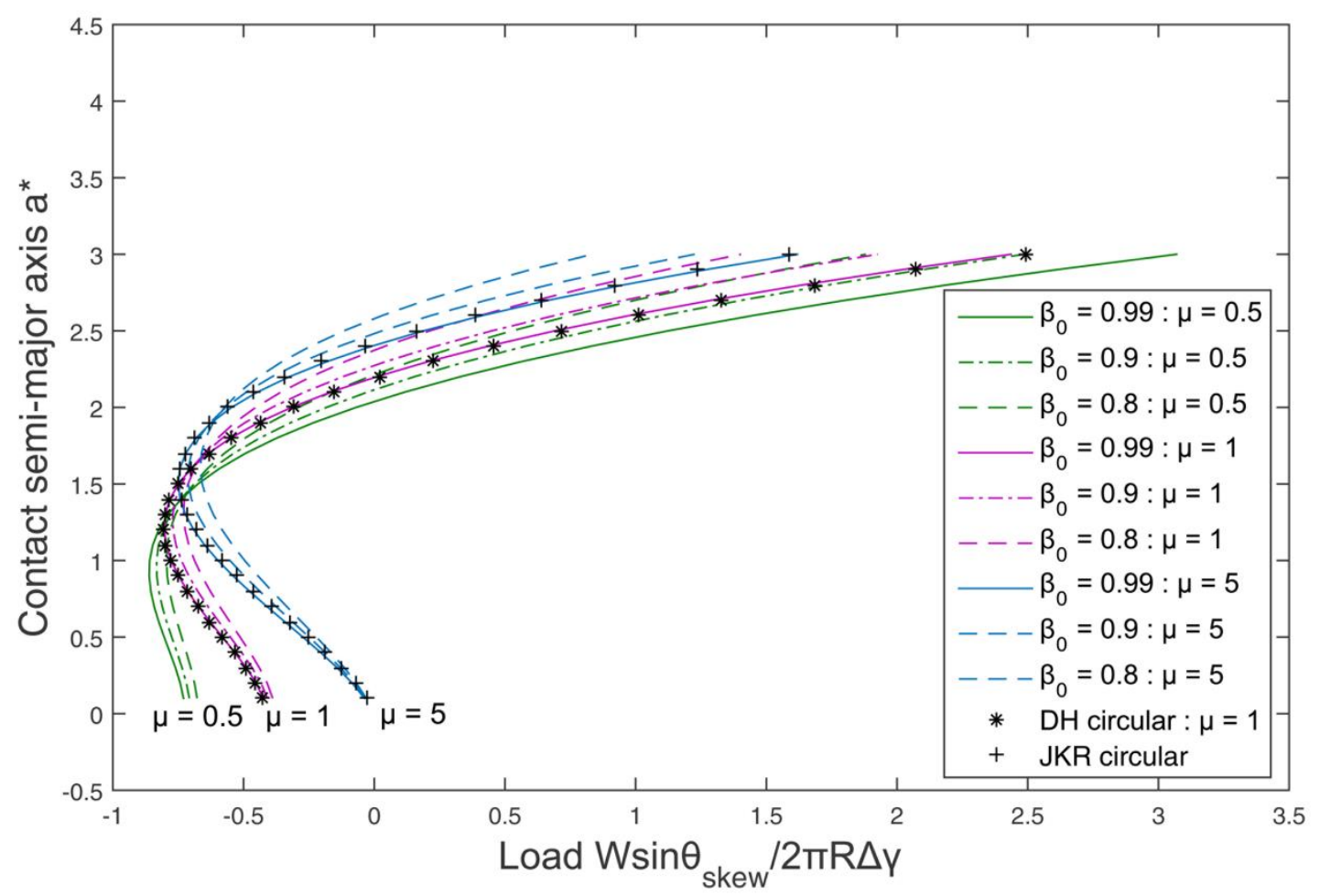

(a)

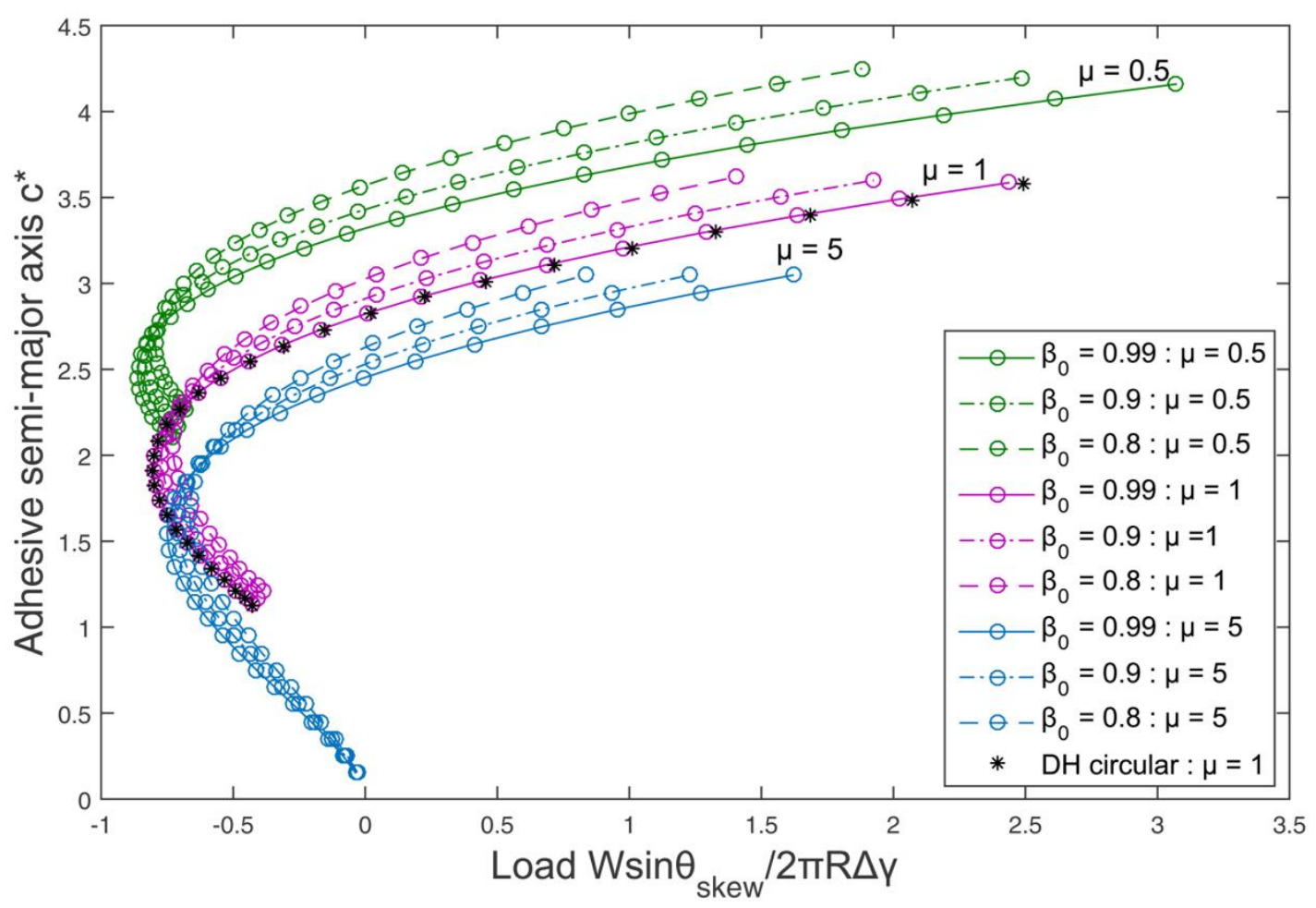

(b) 


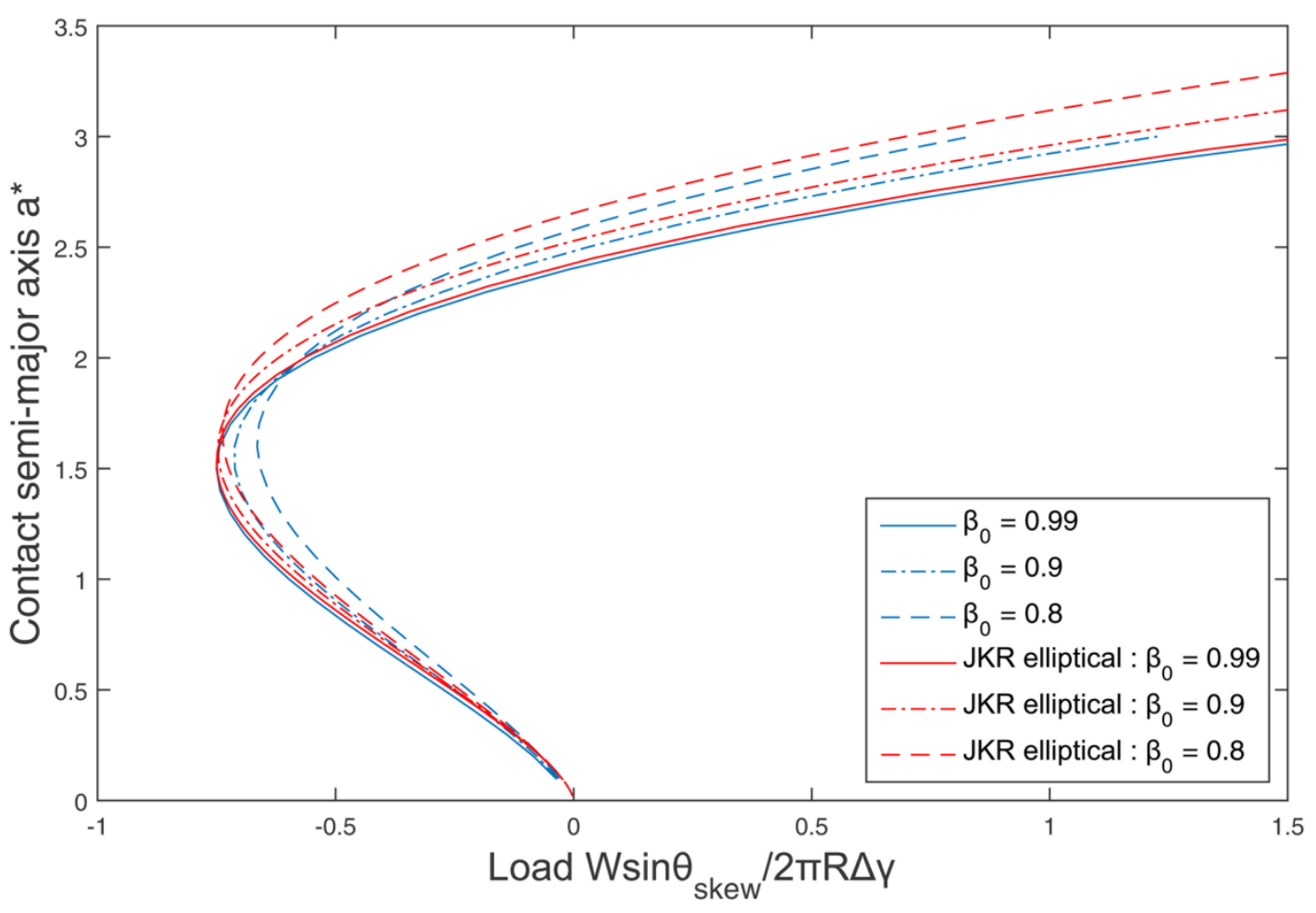

(c)

Fig. 2.5: (a) Variation of the contact semi-major axis $a^{*}$ with the normalised load $W^{*}$ for various values of $\mu$ (b) Variation of the adhesive semi-major axis $c^{*}$ with the normalised load $W^{*}$ for various values of $\mu$ (c) Variation of the contact semi-major axis $a^{*}$ with the normalised load $W^{*}$ for various $\beta_{0}$ values at $\mu=5$.

When the extended DH model adopts the load-dependence behaviour for its adhesive region by using the JKR ellipticity ratio at the pull-off moment, the pull-off force prediction does increase slightly, see Fig. 2.6. The results are still not close to the expected values as the $\mathrm{DH}$ theory models adhesion outside the contact area while the JKR theory models the adhesion only within the contact area. 


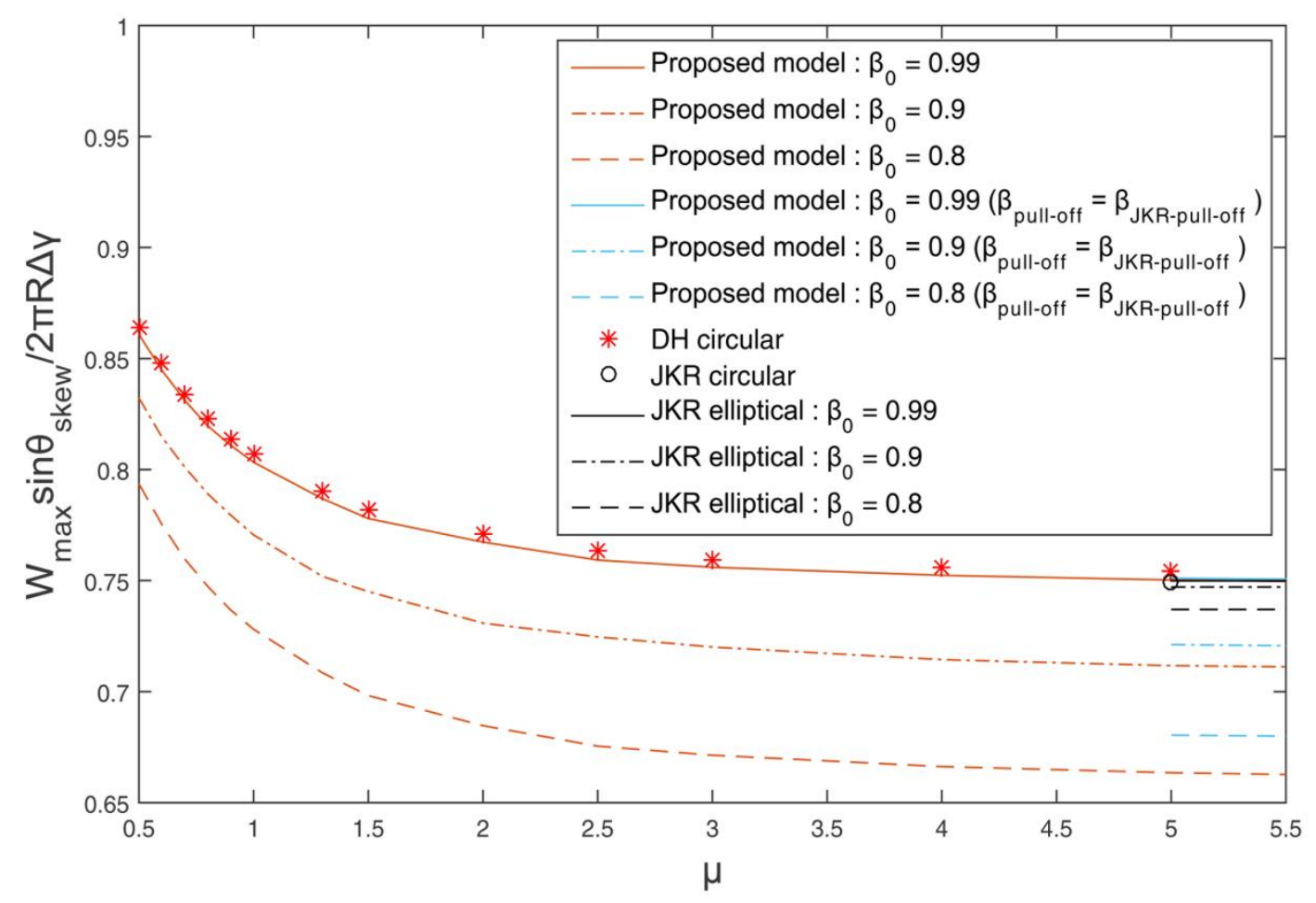

Fig. 2.6: Normalized pull-off force $W_{\max }{ }^{*}$ as a function of the Tabor parameter $\mu$ for various ellipticity ratios $\beta_{0}$.

To summarise, the assumption of a constant ellipticity ratio from the initial to the pull-off force in Eq. (2.5) is suitable only for $\beta_{0}=0.99$. For other types of elliptical contacts, the constant ellipticity ratio assumption in Eq. (2.5) limits the changes in the adhesive region, as shown in Fig 2.7, even though the contact deformations are dominant in the major-axis direction. The load-dependence adhesive region that is assumed by the JKR $\left(\beta_{\text {(pull-off) }} \neq \beta_{0}\right)$ elliptical model [15] allows the adhesive region to change without constraints. As the load varies, the JKR contact changes from an ellipse to a nearly circular shape at the pull-off moment, an observation also supported by the experimental results in [16]. Adhesion that acts at the edge of a nearly circular contact area requires a higher pull-off force for separation compared to the case of a narrow annular elliptical adhesive region, which is the reason why the extended $\mathrm{DH}$ model underestimates the pull-off force prediction. 


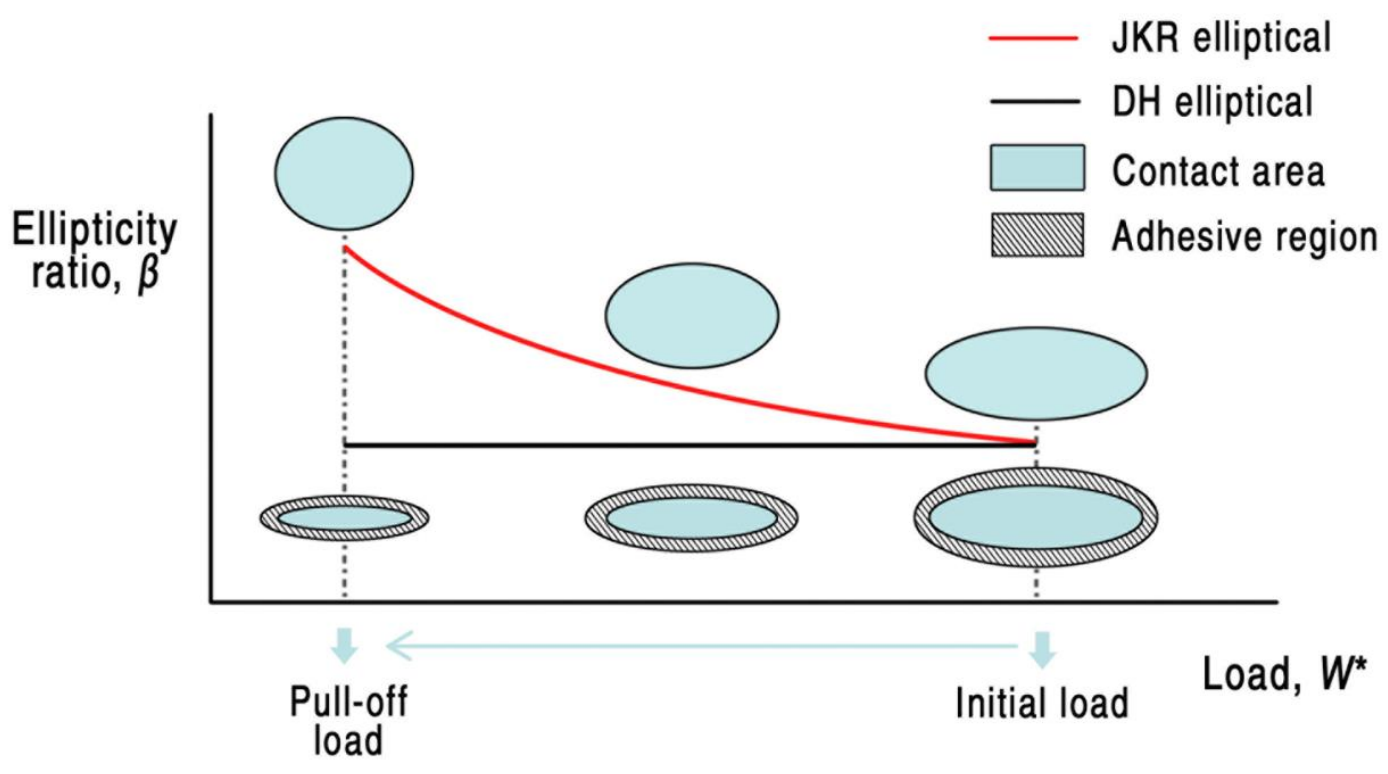

Fig. 2.7: Evolution of the adhesive region and the contact area from the initial loading to the pull-off moment for the JKR elliptical model and the proposed model.

\subsection{Conclusion}

The assumption of identical, fixed ellipticity ratios throughout the contact for the contact and adhesive ellipses that bounded the $\mathrm{DH}$-based adhesive region for elliptical contacts is shown to be unsuitable, as seen in the limiting JKR case where the pull-off force is underestimated. For accurate prediction of a $\mathrm{DH}$ based elliptical contacts, it is important to take into account that both $\beta_{a b}$ and $\beta_{c d}$ do change as the applied load varies. 


\section{Chapter 3}

\section{ADHESION MODELLING OF ELLIPTICAL CONTACTS}

This chapter continues the work on the extended DH model, validating the model using improved, realistic assumptions on the adhesive region, for elliptical contacts with lower skew angles.

\subsection{Introduction}

For an accurate pull-off force prediction, the extended DH model must employ a realistic and accurate assumption of the geometry of the contact and adhesive ellipses, as they affect the shape of the adhesive region. Based on the results obtained in Chapter 2, the ellipticity ratios for contact and adhesive ellipses, $\beta_{a b}$ and $\beta_{c d}$ are shown to change from the initial load to the pull-off moment, given by:

$$
\begin{aligned}
& \beta_{a b_{\text {(pull-off })}} \neq \beta_{0} \\
& \beta_{c d_{(\text {pull-off })}} \neq \beta_{0}
\end{aligned}
$$

At the pull-off moment, the relation between $\beta_{a b}$ and $\beta_{c d}$ can be summarised as:

$$
\beta_{a b_{(p u l l-o f f)}} \neq \beta_{c d(\text { pull-off) }}
$$

Although the load effect on both contact and adhesive ellipses during contact is recognized in Chapter 2 as the correct geometrical behaviour for the $\mathrm{DH}$ based adhesive region, analytical solutions that can be used to calculate both $\beta_{a b}$ and $\beta_{c d}$ values at the pull-off moment are unavailable. Thus, a numerical solution becomes necessary to accurately predict both $\beta_{a b}$ and $\beta_{c d}$ values, for a wide range of adhesive elliptical contacts, see Paper $C$ of Part II.

The development of the extended DH model in Chapter 2 is continued in this chapter, for elliptical contacts with lower skew angles, with a new assumption which the adhesive region is allowed to change without the constraint of a Hertzian contact. Both contact and adhesive ellipses that act as the inner and the outer boundaries of the adhesive region have ellipticity ratios that vary with load, with both having similar values of ellipticity ratio at the beginning of the contact. As the contact progresses to the pull-off moment, both boundaries begin to change in size, with the inner boundary having a different ellipticity ratio compared to the outer boundary, though both boundaries are assumed to keep their elliptical shapes. Chapter 3 focuses on the geometry of the adhesive region at the pull-off moment. The work in Chapter 3 consists of two parts: (1) predicting the geometry of the adhesive region at the pull-off moment by using a numerical 
model, and (2) developing equations to describe the ellipticity ratio of the inner and the outer boundaries of the adhesive region, and also the semi-major axis of the elliptical contact area at the pull-off moment. Results obtained will be compared to the numerical model, other adhesive elliptical models and existing experimental results for validation purposes.

\subsection{Load dependence of DH-based elliptical contacts}

Due to the assumption of a load-dependent adhesive region, several equations of the extended $\mathrm{DH}$ model from Paper $\mathrm{A}$ of Part II are adjusted to $\beta_{a b}$ and $\beta_{c d}$ having different values. These selected equations can be found in Paper C of Part II.

As mentioned before, Chapter 3 focuses on adhesive elliptical contacts at the pulloff moment, hence both $\beta_{a b}$ and $\beta_{c d}$ are now expressed as:

$$
\begin{aligned}
& \beta_{a b}=\beta_{a b_{\text {pull-off }}} \\
& \beta_{c d}=\beta_{c d_{\text {pull-off }}}
\end{aligned}
$$

In Paper $\mathrm{C}$ of Part II, $\beta_{\text {Hertz }}$ is introduced to describe the constant ellipticity ratio obtained from a normal elliptical Hertzian contact. Before the ellipticity ratio changes due to the applied load, the assumption for the adhesive contact at the initial load can be summarised as:

$$
\begin{gathered}
\beta_{0}=\beta_{0 a b}=\beta_{0} c d \\
\beta_{0}=\beta_{H e r t z}
\end{gathered}
$$

Assumptions in Eq. (3.4) are made as an approximation of the behaviour for both contact and adhesive ellipses; though these assumptions are suitable for materials with very low $\mu$ that are close to the DMT domain as these materials barely deform.

The geometry of $\mathrm{DH}$ based adhesive regions due to elliptical contacts can be simulated by a Boundary Element Model (BEM) with a Dugdale approximation for the adhesive stress, developed by [22], utilising the Conjugate Gradient Method (CGM). The numerical model is suitable for solving non-linear contact problems such as adhesive elliptical contacts where the contact geometry is unknown a priori. In the method, the contribution of each element in the pressure and the deformation profiles is considered separately. The CGM algorithm is shown in Fig. 3.1. Detailed equations of the numerical model are shown in Paper C of Part II. 


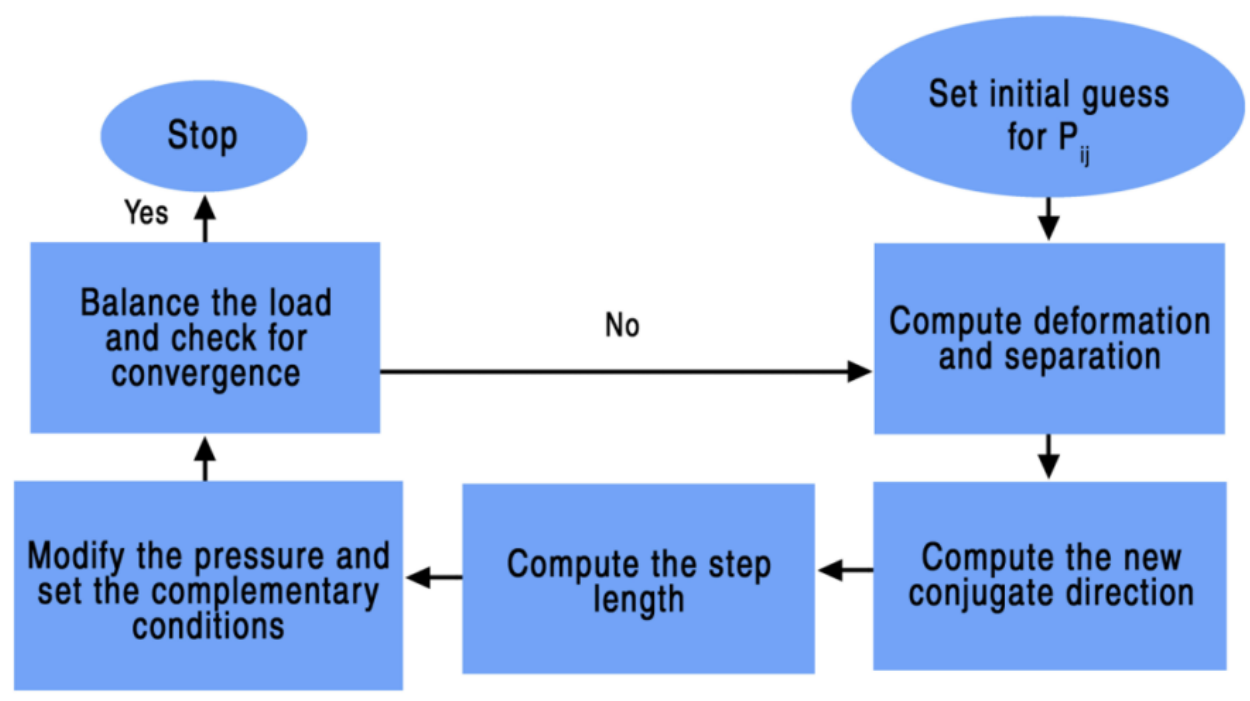

Fig. 3.1: CGM based algorithm for adhesive contacts [22].

Numerical simulations are conducted for $\beta_{0}$ values of $0.3\left(\theta_{\text {skew }}=43.63^{\circ}\right), 0.4\left(\theta_{\text {skew }}\right.$ $\left.=53.14^{\circ}\right), 0.6\left(\theta_{\text {skew }}=68.51^{\circ}\right)$ and $0.8\left(\theta_{\text {skew }}=80.45^{\circ}\right)$, within the range of $0.5 \leq \mu \leq 4$. Results from the numerical simulations are obtained using the element number, $N$ along the major and the minor axes of $N=2048$ for $0.5 \leq \mu<2$ (the domain size is 2048 elements $\times 2048$ elements) and $N=4096$ for $2 \leq \mu \leq 4$ (the domain size is 4096 elements $\mathrm{x}$ 4096 elements). In all cases, the calculation domain is set six times larger than the contact domain. An increased resolution is required for larger $\mu$ values due to the small adhesive zone outside the contact. These values are set after repeating the numerical computations using various element numbers and extension factors for the contact domain to get the optimal numerical parameters for all contact conditions. The accurate prediction of $\beta_{a b}$ and $\beta_{c d}$ values at the pull-off moment uses a negative load input in the numerical algorithm; this negative load represents the pull-off force required to separate the surfaces. The pull-off force is determined by the greatest negative load that first converges within 200 iterations.

An example of the numerical model's results on predicting adhesive elliptical contacts at the pull-off moment is shown in Fig. 3.2. The results are obtained using the contact parameters in Table 3.1 as the input. Fig. 3.2a shows the image of threedimensional pressure profile within the computation domain with 2048 elements along the major and the minor axes while Fig. 3.2b and Fig. 3.2c show the resulting pressure distribution along the semi-major axis and semi-minor axis. The selected points of $a$ and $b$ are obtained from the semi-major and semi-minor axes of the contact ellipse while the points of $c$ and $d$ are obtained from the semi-major and semi-minor axes of the adhesive ellipse. 
Table 3.1: Parameter of the adhesive elliptical contact at the pull-off moment.

\begin{tabular}{|l|l|l|}
\hline Parameters & Values & Unit \\
\hline Predicted pull-off force & 75 & $\mathrm{nN}$ \\
\hline Fiber diameter & 3.5 & $\mathrm{Mm}$ \\
\hline Skew angle, $\theta_{\text {skew }}$ & 43.63 & $\circ$ \\
\hline Hertzian ellipticity ratio, $\beta_{0}$ & 0.3 & - \\
\hline Tabor parameter, $\mu$ & 0.5 & - \\
\hline Number of elements along major and minor axes, $N$ & 2048 & - \\
\hline
\end{tabular}

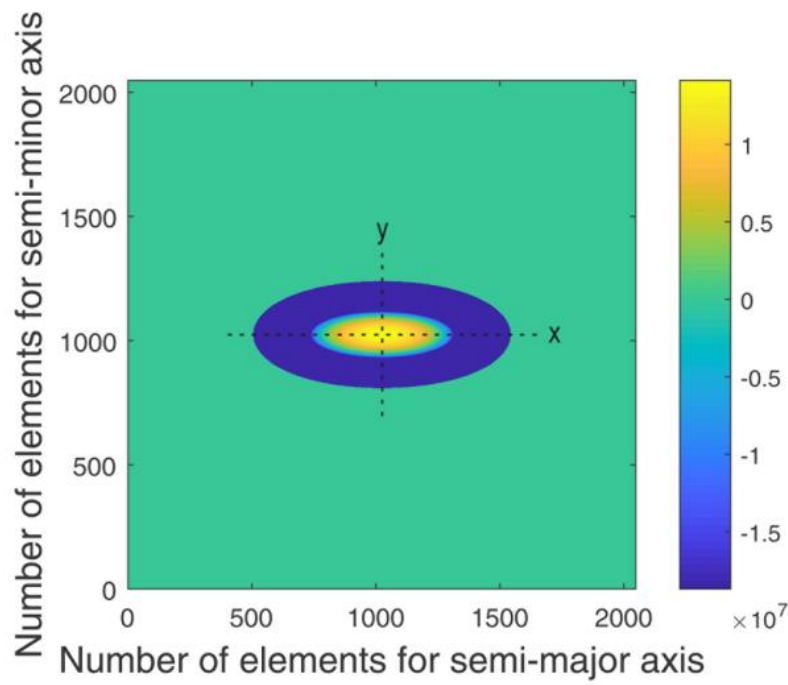

(a)

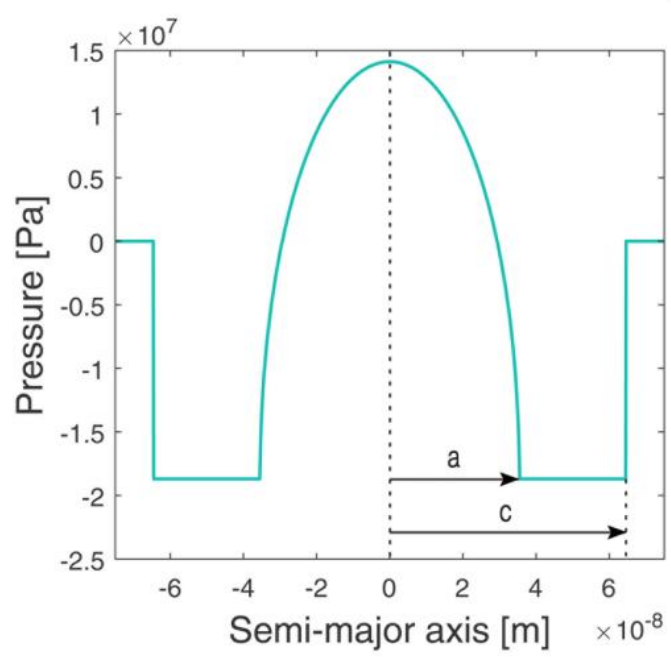

(b)

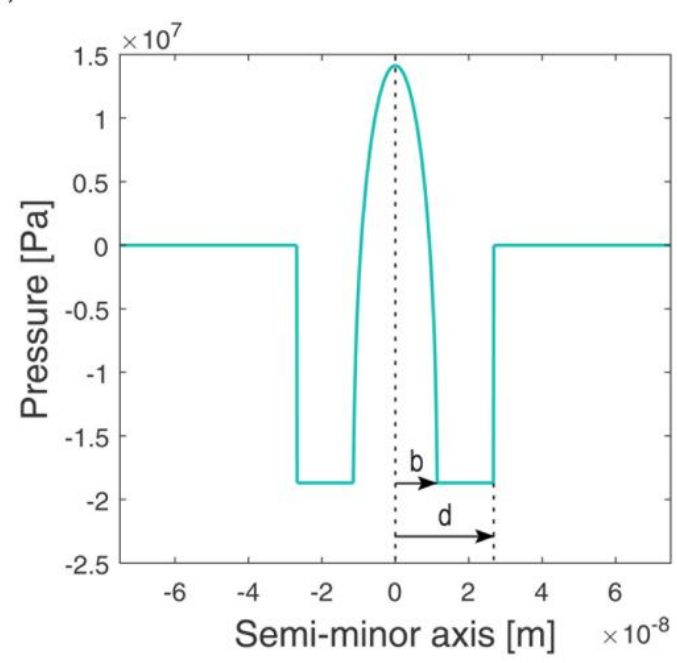

(c)

Fig. 3.2: Numerical solution for the elliptical contact (a) three-dimensional pressure profile for the elliptical contact (b) stress distribution along the semi-major axis and (c) stress distribution along the semi-minor axis. 
Using the data from the numerical model, equations that can predict the values of $\beta_{a b}$ and $\beta_{c d}$ at the pull-off moment, as a function of $\mu$ and $\theta_{\text {skew }}$ are obtained. These ellipticity ratio equations at the pull-off moment are given as:

$$
\begin{aligned}
& \beta_{a b}=0.3899 \theta_{\text {skew }}{ }^{1.7669}+0.0829 \mu^{0.3316}+0.0233 \\
& \beta_{c d}=0.4139 \theta_{\text {skew }}{ }^{1.5837}+0.7199 \mu^{-0.0074}-0.5783
\end{aligned}
$$

Using $\beta_{a b}$ from Eq. (3.5a), the equation to predict the semi-major axis of the contact ellipse at the pull-off moment, $a$ is given as:

$$
a=\left(\frac{R^{2} \Delta \gamma}{E^{*}}\right)^{1 / 3}\left(\sinh \left(1.0499 \beta_{a b}{ }^{-0.1553}-0.0648 \mu^{-1.1945}\right)\right)^{2}
$$

Eq. (3.5) and Eq. (3.6) are then incorporated into the extended DH model to calculate the semi-major axis of the adhesive ellipse at the pull-off moment, $c$ and the scaling factor $v$, by solving Eq. (31) and Eq. (35) in Paper A of Part II. With this information, the pull-off force can be calculated using Eq. (14) in Paper C of Part II.

\subsection{Results and discussion}

For the curve-fitting of the ellipticity ratio data at the pull-off moment, there are many mathematical functions that can be considered to represent the data. However, by referring to the pull-off force behaviour of an adhesive circular contact as discussed in [12], it is clear how the contact should behave. The curve-fitted equations in Eq. (3.5) are shown to predict accurately the $\beta_{a b}$ and $\beta_{c d}$ values, as shown in Fig. 3.3a and Fig 3.3b. The values of $\beta_{a b}$ increase rapidly at low $\mu$ values that are close to the DMT domain. Materials that are considered close to the DMT domain barely deform along the semimajor axis direction, as shown by the close value of $\beta_{a b}$ to $\beta_{0}$. The rapid increase in $\beta_{a b}$ eventually slows down as the curve is within the intermediate domain and becomes nearly stable as it approaches the JKR domain. Closed to the JKR domain, the highly elastic materials are easily deformed, resulting in high $\beta_{a b}$ values. This behaviour is represented well by Eq. (3.5a). As for the behaviour of $\beta_{c d}$, there is no reference available on how the adhesive ellipse might behave. Solely based on the trend of the numerical data, it is shown that the values of $\beta_{c d}$ decrease at a nearly constant manner, indicating that the effect of $\mu$ on $\beta_{c d}$ is not dominant. When compared with the numerical model, the maximum errors of $\beta_{a b}$ and $\beta_{c d}$ predictions using Eq. (3.5) are $2.67 \%$ for $\beta_{a b}$ at $\beta_{0}=0.3$, and $0.76 \%$ for $\beta_{c d}$ at $\beta_{0}$ $=0.4$, whereas the minimum errors are calculated as $0.124 \%$ at $\beta_{0}=0.8$ and $0.007 \%$ at $\beta_{0}$ $=0.3$, for $\beta_{a b}$ and $\beta_{c d}$, respectively. As for the errors of $a_{\text {pull-off }}$ prediction using Eq. (3.6), the maximum error is $2.87 \%$ at $\beta_{0}=0.4$, while the minimum error is $0.15 \%$ at $\beta_{0}=0.3$. 

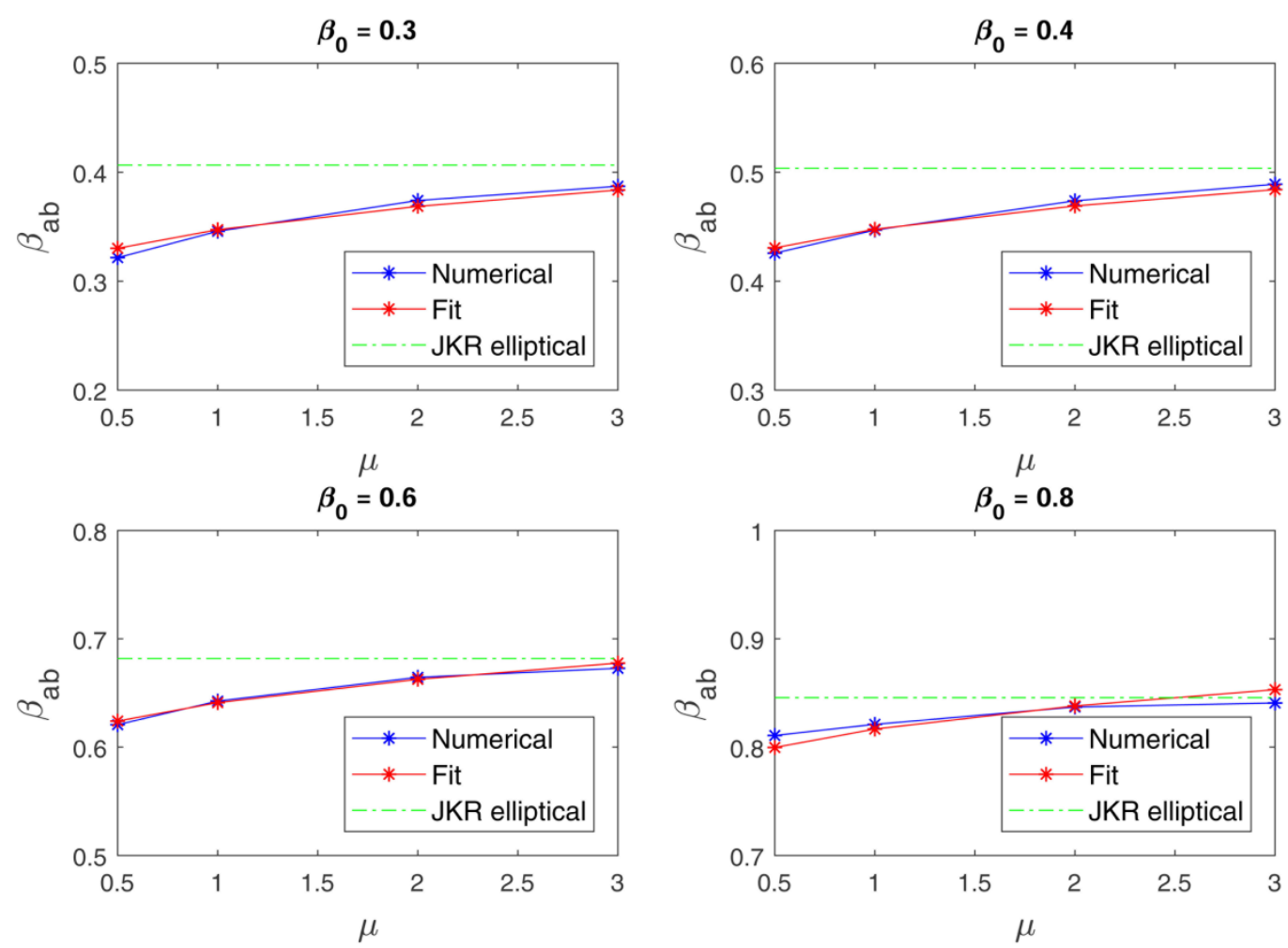

(a)
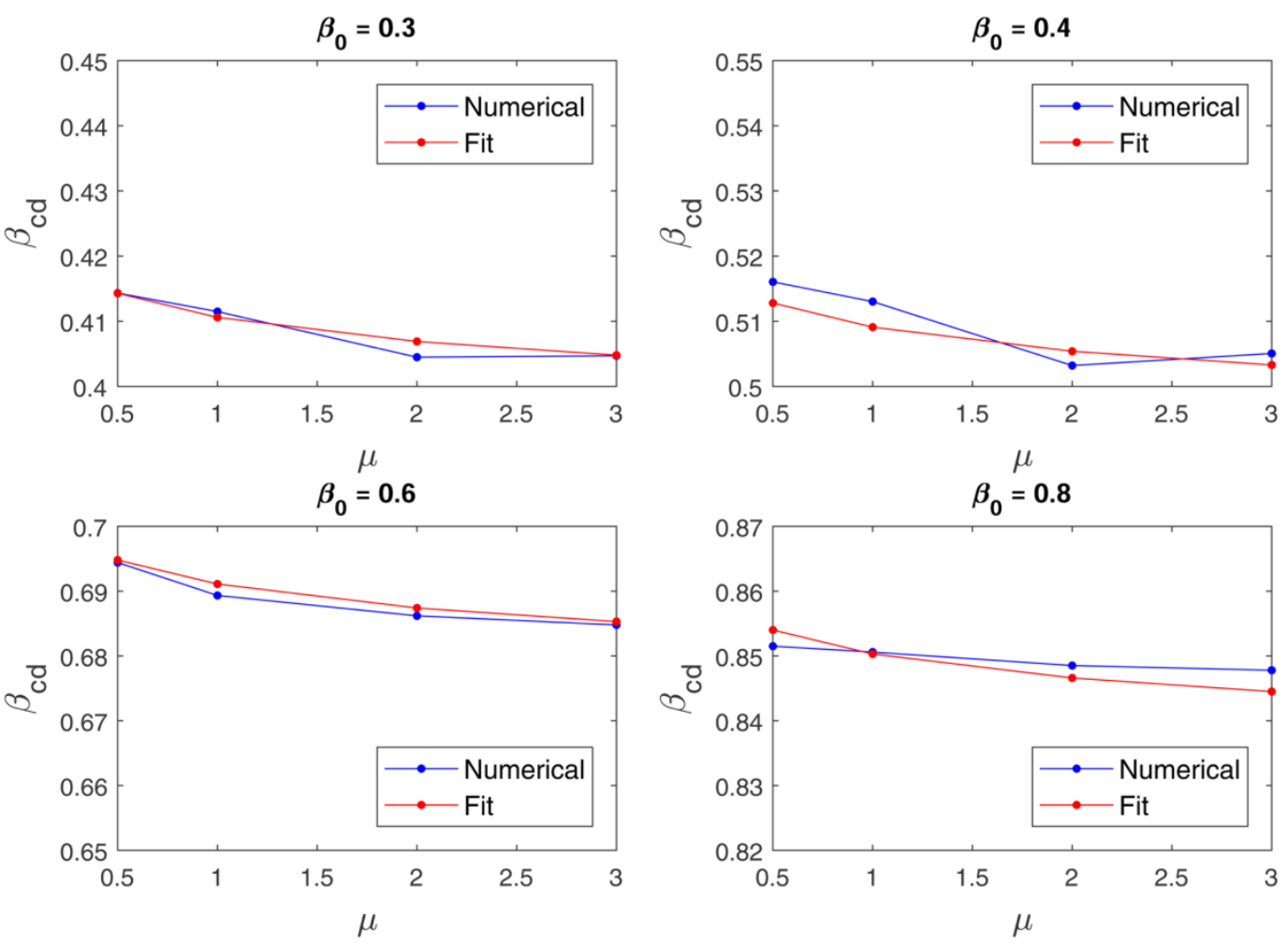

(b)

Fig. 3.3: (a) Ellipticity ratio of the contact ellipse for the numerical model and the curvefitted equation at the pull-off moment for various $\beta_{0}$ values (b) Ellipticity ratio of the adhesive ellipse for the numerical model and the curve-fitted equation at the pull-off moment for various $\beta_{0}$ values. 
The values of non-dimensionalised contact semi-major axis at the pull-off moment $a^{*}$ from Eq. (3.6) and the resulting adhesive semi-major axis, $c^{*}$ are plotted in Fig. 3.4a, alongside the numerical results. Values from Eq. (3.5) and Eq. (3.6) are used in the extended DH model to calculate $c^{*}$ values. The $a^{*}$ values from both numerical results and Eq. (3.6) are similar for all $\beta_{0}$ values, while the $c^{*}$ values obtained from the solution of Eq. (3.5) and Eq. (3.6) are higher from the numerical results, as expected. The higher $c$ prediction is set to compensate the decreasing values of adhesive stresses, as opposed to constant adhesive stresses by the MD model in the adhesive region, as shown in Fig. 3.4b. This is to ensure that both MD and DH models have the same work of adhesion. In general, the adhesive zone in the semi-major axis direction becomes smaller as $\mu$ approaches the JKR domain. For lower $\mu$, the pull-off forces predicted by both the extended DH model and the numerical model are close even though the fitted $c^{*}$ is larger than the $c^{*}$ predicted numerically. The difference between the two $c^{*}$ values has little influence on the pull-off force prediction, as the results of the two models are close. However, the numerical errors become significant at higher $\mu$ values that are close to or already in the JKR domain, especially for low $\beta_{0}$ values due to a very small adhesive zone. At very high $\mu$, the adhesive zone is so small that the extended $\mathrm{DH}$ model cannot model such behaviour accurately. Hence, the pull-off forces predicted by the extended DH model have large errors in comparison with the numerical results. 

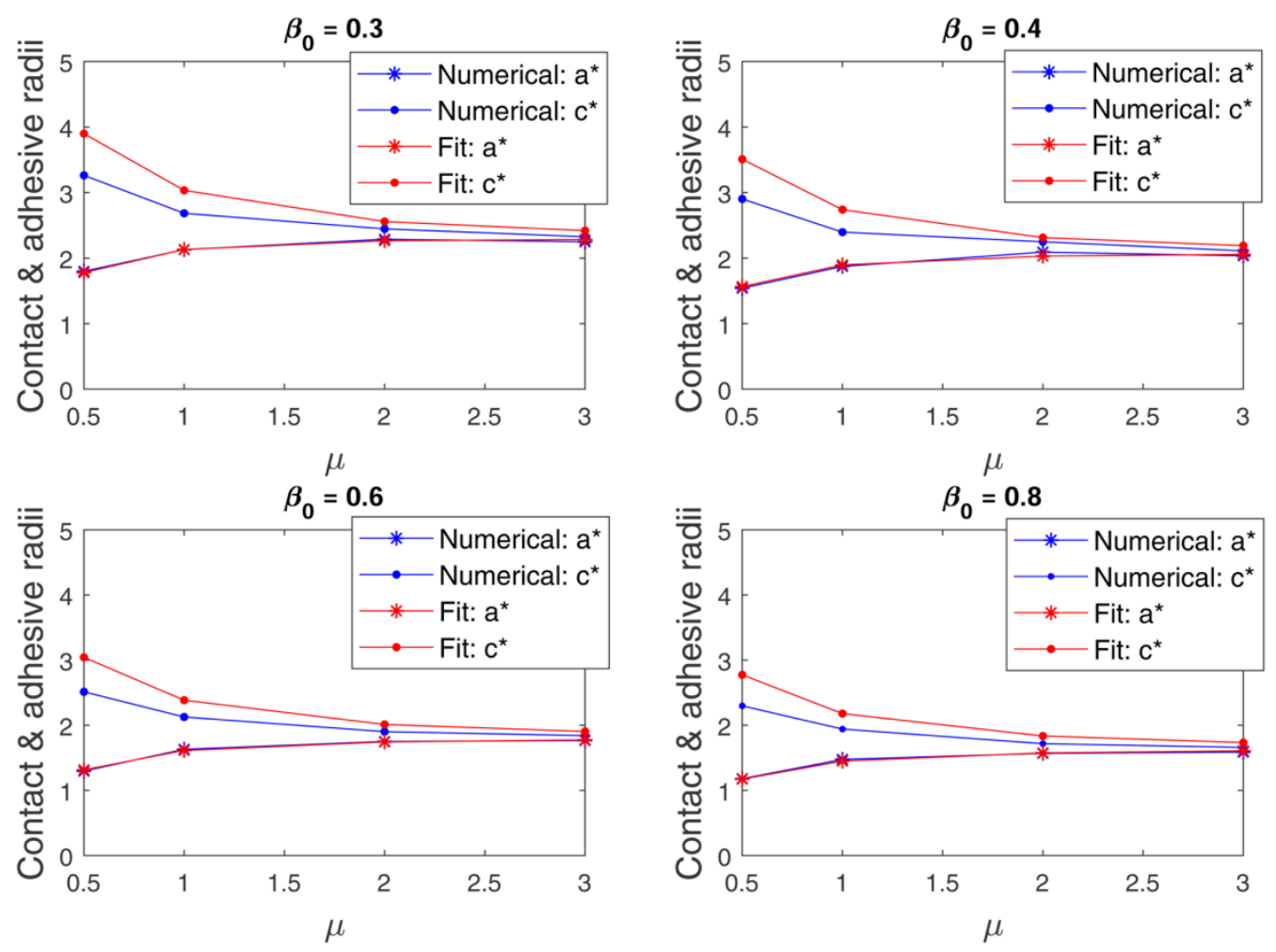

(a)

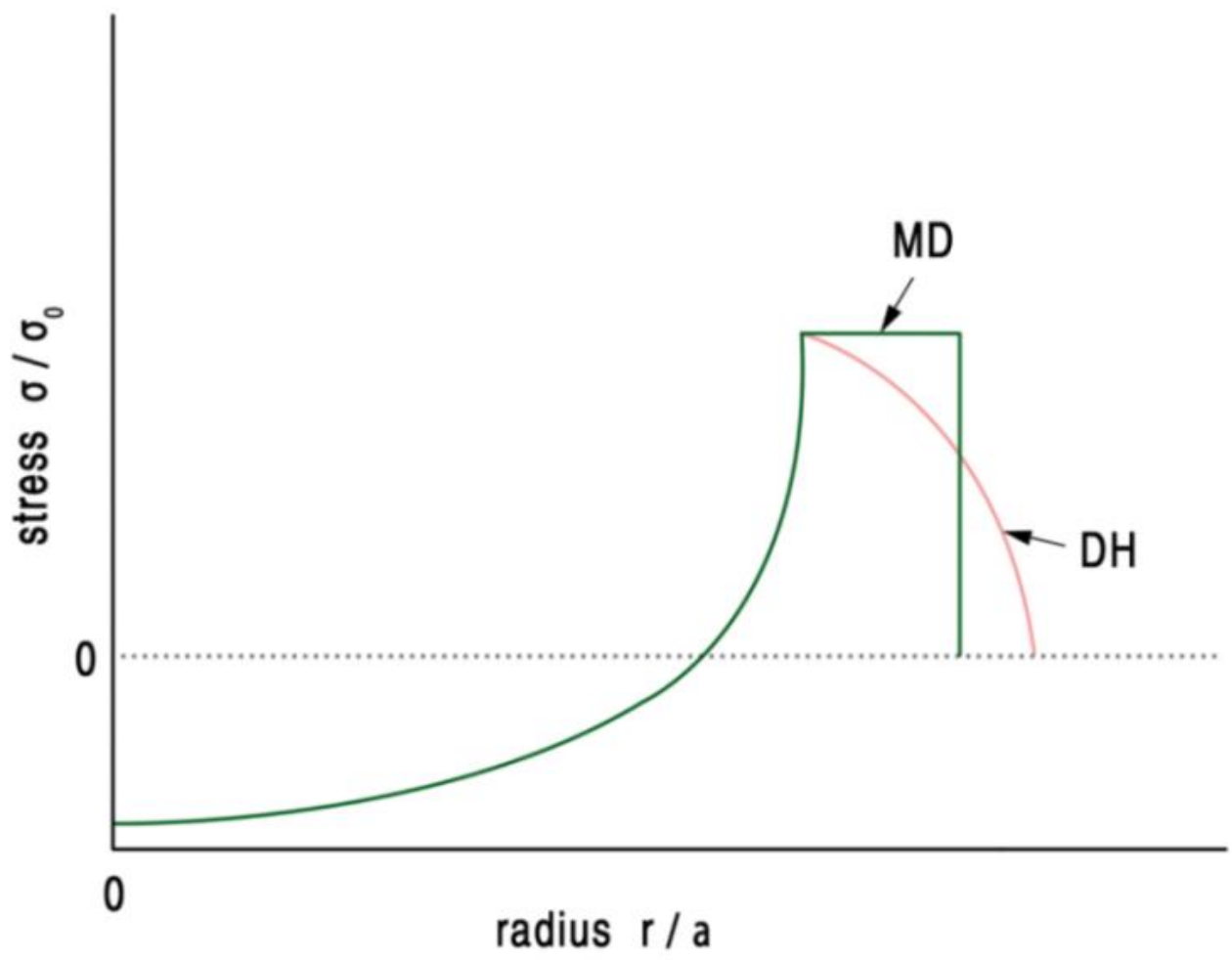

(b)

Fig. 3.4: (a) Non-dimensionalised semi-major axis of the contact ellipse for the numerical model and the curve-fitted equations at the pull-off moment for various $\beta_{0}$ values (b) Graphical representation of adhesive stresses distribution by both DH and MD models. 
The extended DH model incorporated with Eq. (3.5) and Eq. (3.6) is validated by comparing its pull-off force results with those of the JKR elliptical model, the numerical simulation and the experimental results in [16], as shown in Fig. 3.5. Eq. (3.5) and Eq. (3.6) are incorporated in the extended $\mathrm{DH}$ model to predict $c^{*}$ and the scaling factor $v$ at the pull-off moment, which are then used to calculate the pull-off forces. For $\beta_{0}$ values of 0.3 and 0.4 , the curves for both numerical and extended $\mathrm{DH}$ model are similar for low $\mu$ up to $\mu=1$, where the numerical pull-off forces keep on decreasing while the pull-off force values of the extended $\mathrm{DH}$ model begin to increase. For $\beta_{0}$ value of 0.8 , the extended $\mathrm{DH}$ model predicts higher pull-off forces near the DMT domain, though before $\mu=2$ the pull-off forces of the numerical model start to exceed the extended $\mathrm{DH}$ results.
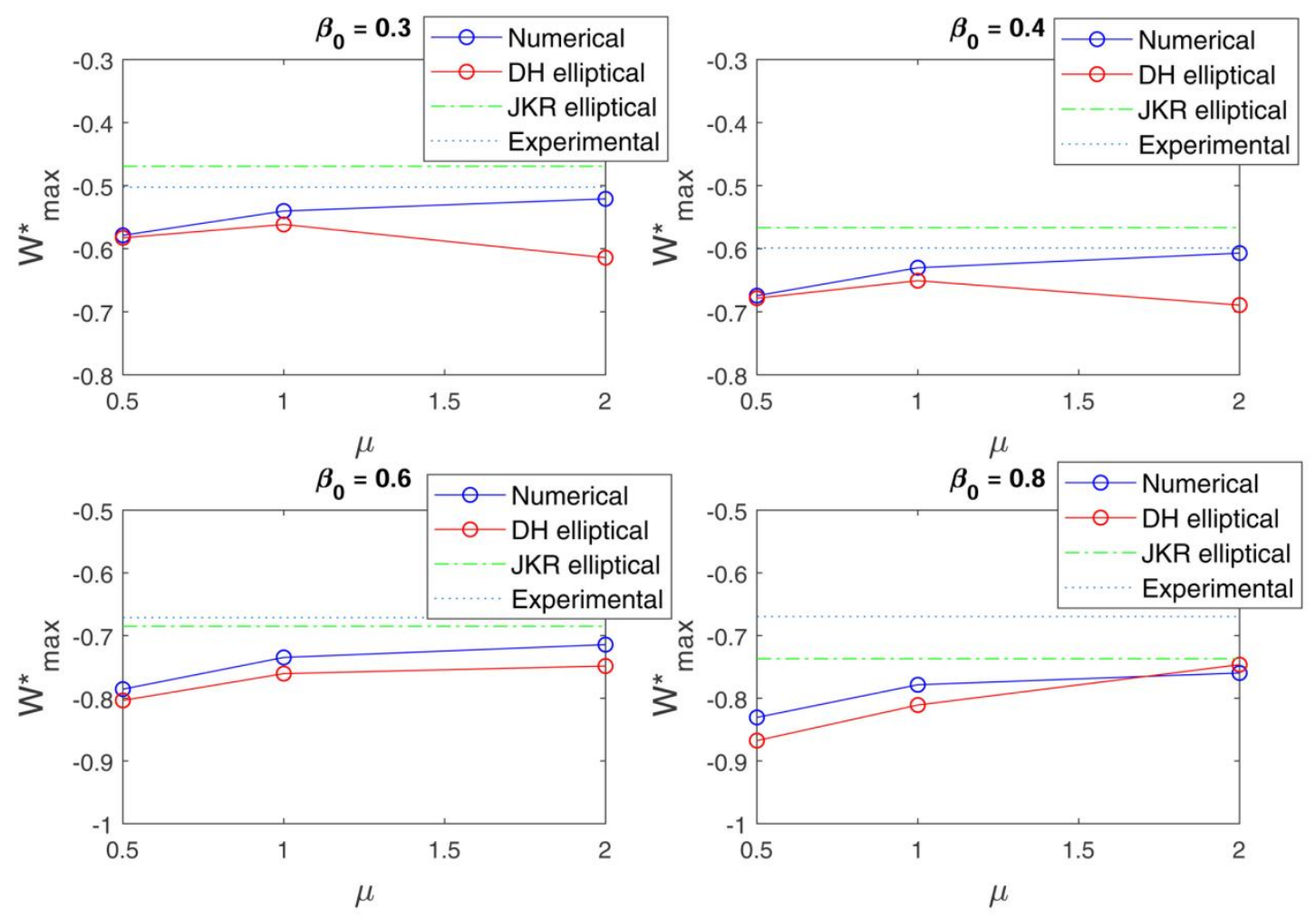

Fig. 3.5: Pull-off force prediction between the numerical model, the extended $\mathrm{DH}$ model, the JKR elliptical model and the existing experimental results for various $\beta_{0}$ values.

Some of the results in Fig. 3.5 are replotted in Fig. 3.6 with the pull-off force as a function of $\theta_{\text {skew. }}$. The curves for $\mu$ values of 0.5 and 1 are shown to have behaviour similar to that of the JKR elliptical model; the curve of $\mu=1$ follows the behaviour of the JKR results very closely, in contrast to the curve of $\mu=0.5$, signifying that it is very close to the JKR domain. However, for the curve of $\mu=2$, its behaviour is totally different from the JKR result. This can be an indicator that at $\mu=2$, the contact is already within the JKR domain. 
This situation is further proved by the ratio of $c_{\text {num }}{ }^{*}$ to $a_{\text {num }}{ }^{*}$ in Table 3.2, calculated from the numerical results. At $\mu=2$, the values of $c_{\text {num }} / a_{\text {num }}{ }^{*}$ are very close to 1 , especially for lower $\beta_{0}$ values, a sign that the adhesive region outside the contact is very small, which is the characteristic of the JKR model. To conclude, the JKR domain is larger for elliptical contacts than for circular contacts, since the JKR domain starts at much lower $\mu$ value than 5 , which is the application limit for the JKR model of circular contacts.

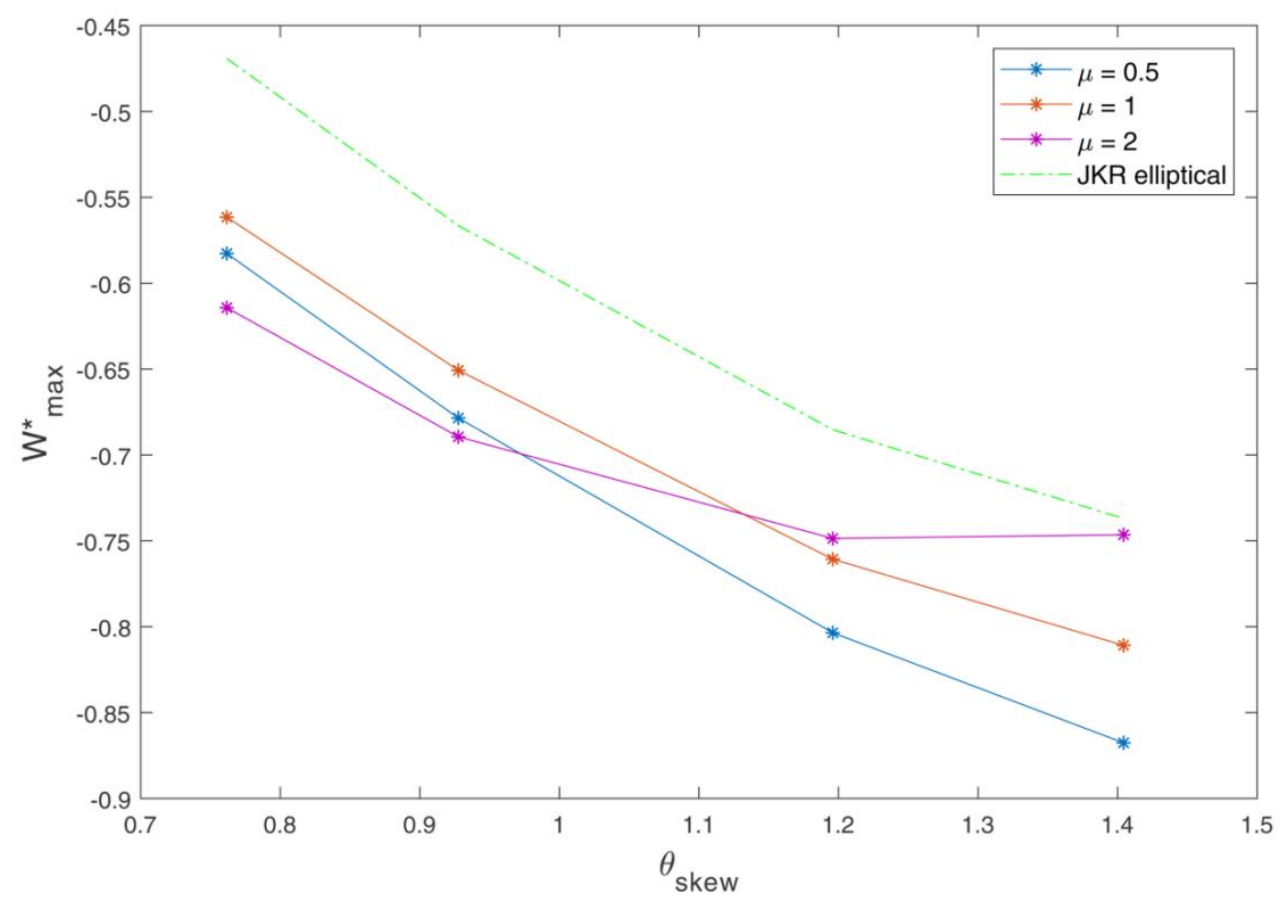

Fig. 3.6: Pull-off forces as a function of $\theta_{\text {skew }}$ for $\mu$ values of $0.5,1$ and 2, in comparison with the JKR elliptical model.

Table 3.2: Ratio of $c_{\text {num }}{ }^{*}$ to $a_{\text {num }}{ }^{*}$ for various contact conditions.

\begin{tabular}{|l|l|l|l|l|}
\hline \multirow{3}{*}{$\mu$} & \multicolumn{4}{|l|}{$c_{\text {num }}{ }^{\star} a_{\text {num }}{ }^{*}$} \\
\cline { 2 - 5 } & $\beta_{0}=0.3$ & $\beta_{0}=0.4$ & $\beta_{0}=0.6$ & $\beta_{0}=0.8$ \\
\hline 0.5 & 1.8146 & 1.8837 & 1.9379 & 1.9477 \\
\hline 1 & 1.2606 & 1.2793 & 1.3039 & 1.3157 \\
\hline 2 & 1.0692 & 1.0757 & 1.0863 & 1.0947 \\
\hline 3 & 1.0340 & 1.0371 & 1.0425 & 1.0459 \\
\hline
\end{tabular}




\subsection{Conclusion}

The work on the extended DH model has been continued to include the assumption of an adhesive region with boundaries that vary with load, for an accurate pull-off force prediction. The geometry of the annular adhesive region at the pull-off moment is obtained from a Dugdale-based numerical model. These numerically-obtained values are curvefitted to find the equations to predict the ellipticity ratios for both ellipses that bounded the adhesive region at the pull-off moment and the semi-major axis of the contact ellipse, and then incorporated into the extended $\mathrm{DH}$ model. The incorporated equations of the ellipticity ratio and the contact semi-major axis at the pull-off moment allow the extended $\mathrm{DH}$ model to accurately predict the pull-off force in its working domain of $0.5 \leq \mu<2$, for various elliptical contacts. It is shown that the validity domain for the JKR model is expanding and dominating adhesive elliptical contacts. 



\section{Chapter 4}

\section{ADHESION MAP FOR ADHESIVE ELLIPTICAL CONTACTS}

The results in Chapter 3 show that for adhesive elliptical contacts the JKR model is important. The DH model transitions to the JKR model at lower $\mu$ values than the typical limit for adhesive circular contacts, regardless of the $\theta_{\text {skew }}$ values. However, the application limit between the DH and the JKR model for elliptical contacts is unclear. In this chapter, the construction of an adhesion map for elliptical contacts will be explained; the adhesion map can guide the selection of the suitable contact model for a specific contact condition.

\subsection{Introduction}

For adhesive circular contacts, it has been concluded from [14] that adhesion is best described by the DMT model [10] for $\mu<0.1$ and the JKR model [7] for $\mu>5$. For $0.1<\mu<$ 5 , adhesion can be described either by the MD model [11] or by the DH model [12]. As for the elliptical contacts, there are three contact models that can describe adhesion: (1) the DMT-based model [15], (2) the JKR-based model [18] and (3) the DH-based model from Paper A of Part II. Unlike the circular contact, there is no established criterion on the selection of the elliptical contact models. In Chapter 3, the results show that the JKR elliptical model is valid for smaller $\mu$ values than $\mu=5$, more so as the skew angle becomes smaller. This shows that the JKR domain becomes larger as the contact area becomes more elliptical. To define the validity domain of the different adhesive elliptical models, a map can be utilized to show the domain of each model graphically. This adhesion map can provide guidance and criteria for selecting a suitable contact model from existing elliptical contact models for a specific contact type.

In Chapter 4, the construction of the adhesion map for adhesive elliptical contacts will be described; the $\mathrm{DH}$ elliptical model becomes the base contact model to compare with other elliptical contact models. The application of the adhesion map is also demonstrated; for a specific contact condition, the chosen adhesive contact model is compared with existing experimental results. 


\subsection{Adhesion map}

\subsubsection{Construction}

The construction of the adhesion map for elliptical contacts is similar to the adhesion map for the circular contacts [13]; the adhesion map for adhesive elliptical contacts is plotted using the coordinate of $\mu$ from Eq. (35) and $W^{*}$ from Eq. (36), both from Paper A of Part II. The adhesion map for elliptical contacts is based on the DH elliptical model from Paper $\mathrm{A}$ of Part II because the MD model is not available for elliptical contacts.

The adhesion map for elliptical contacts is shown in Fig. 4.1. Using the DH elliptical model as the base model for comparison, the adhesion map is plotted for the limited range of $0.5 \leq \mu \leq 4$; these $\mu$ values are selected due to the result from Paper $\mathrm{C}$ of Part II which found that the working domain for the $\mathrm{DH}$ model is smaller than the working domain of the $\mathrm{DH}$ circular model. Because of the relatively high $\mu$ values, the adhesion map covers only the domains of the $\mathrm{DH}$ elliptical model and the JKR elliptical model. As mentioned in Chapter 1 , the skew angle for elliptical contacts can vary, with $0^{\circ}<\theta_{\text {skew }}<90^{\circ}$. The adhesion map in Fig. 4.1 is plotted for three $\theta_{\text {skew }}$ of $45^{\circ}, 60^{\circ}$ and $89.5^{\circ}$.

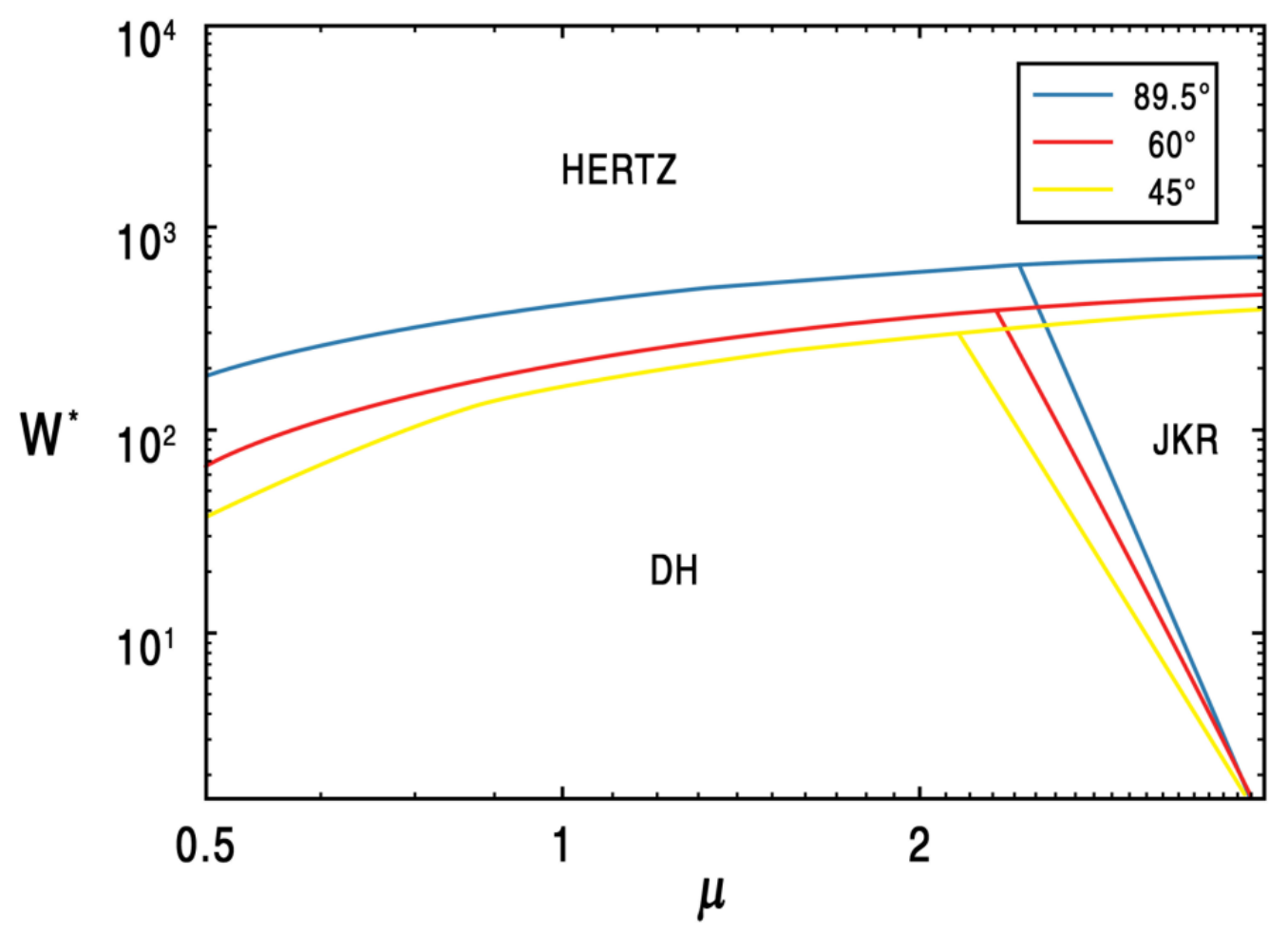

Fig 4.1: Adhesion map for elliptical contacts. 


\subsubsection{The Hertz domain}

Adhesion force can be ignored at high load, which means that the Hertz theory is applicable. The Hertz limit can be drawn using the limit of:

$$
\left|W_{D H} / W\right|<\zeta
$$

where $W$ is taken from Eq. (25) of Paper A of Part II and the DH-based adhesive force $W_{D H}$ is obtained by subtracting Eq. (10) from Eq. (25), both from Paper A of Part II, which results in:

$$
W=-\frac{4 E^{*}}{3 R F_{1}}\left\{v\left[(c d)^{3 / 2}-(a b)^{3 / 2}\right]\right\}
$$

The value of $\zeta$ is chosen arbitrarily. For this adhesion map, the Hertz limit is drawn for $\zeta=$ 0.05 , similar to the value used in the adhesion map for circular contacts [13]. The chosen limit of $\zeta=0.05$ means that the contact is regarded as adhesive when the pull-off force is more than five percent of the total load.

\subsubsection{The JKR domain}

The JKR domain is valid for contact surfaces that are highly elastic. The limit for the JKR domain can be expressed as:

$$
|\delta / h|>\eta
$$

From Paper A of Part II, $\delta$ is from Eq. (14a) and $h$ is from Eq. (27). The limit of the JKR zone is chosen to be $\eta=20$, which is similar to the value used to limit the JKR zone in the adhesion map in [13], see Fig. 1.5.

\subsubsection{Application}

To demonstrate the application of the adhesion map, experimental results from [23] are used, where the pull-off force for the fiber contacts with various $\theta_{\text {skew }}$ values are obtained using the Atomic Force Microscopy (AFM). Experiments on the fiber-on-fiber contacts use aramid fibers with properties as listed in Table 4.1. The pull-off force experiments are conducted at $\theta_{\text {skew }}$ of $45^{\circ}, 60^{\circ}$, and $89.5^{\circ}$, similar to the $\theta_{\text {skew }}$ values of the curves plotted in the adhesion map. 
Table 4.1: Mechanical properties for the aramid (Twaron) fiber.

\begin{tabular}{|l|l|l|l|}
\hline Property & Value & Unit & Ref \\
\hline Axial elastic modulus, $E_{\text {axial }}$ & 109 & $\mathrm{GPa}$ & {$[23]$} \\
\hline Transverse elastic modulus, $E_{\text {trans }}$ & 1.6 & $\mathrm{GPa}$ & {$[23]$} \\
\hline Poisson's ratio, $\vartheta$ & 0.33 & - & {$[23]$} \\
\hline Fiber radius & 6 & $\mu \mathrm{m}$ & {$[23]$} \\
\hline Work of adhesion & 65.38 & $\mathrm{~mJ} / \mathrm{m}^{2}$ & {$[23]$} \\
\hline Intermolecular distance, $z_{0}$ & 5.1 & $\AA$ & {$[24]$} \\
\hline
\end{tabular}

The data from Table 4.1 is used as the input to calculate $\mu$, using Eq. (35) from Paper A of Part II. Table 4.2 lists all the calculated $\mu$ values for the corresponding $\theta_{\text {skew. }}$ Using the non-dimensional values of the pull-off force and the $\mu$ values on the adhesion map developed in Section 4.2.1, the suitable model for the contact is the JKR elliptical contact model. Figure 4.2 shows the comparison between the measured pull-off force and the forces obtained by the JKR elliptical contact model. It can be seen that the discrepancy between the two results is more significant as the value of $\theta_{\text {skew }}$ decreases. This trend is similar to another comparison of measured and calculated data for JKR contacts [16,17]. The JKR elliptical model assumes that the adhesive contacts are between smooth surfaces [15]. On the other hand, it has been discussed in [25] that surface roughness plays an important role in measuring the adhesion force. Even an increase of irregularities just a few nanometers within the range of 1 to $10 \mathrm{~nm}$ would result in the adhesion force decreasing by more than an order of magnitude. 
Table 4.2: Tabor parameter for various $\theta_{\text {skew }}$ values.

\begin{tabular}{|l|l|l|l|}
\hline$\theta_{\text {skew }}$ & $45^{\circ}$ & $60^{\circ}$ & $89.5^{\circ}$ \\
\hline Tabor parameter, $\mu$ & 6.9741 & 6.5186 & 6.2135 \\
\hline
\end{tabular}

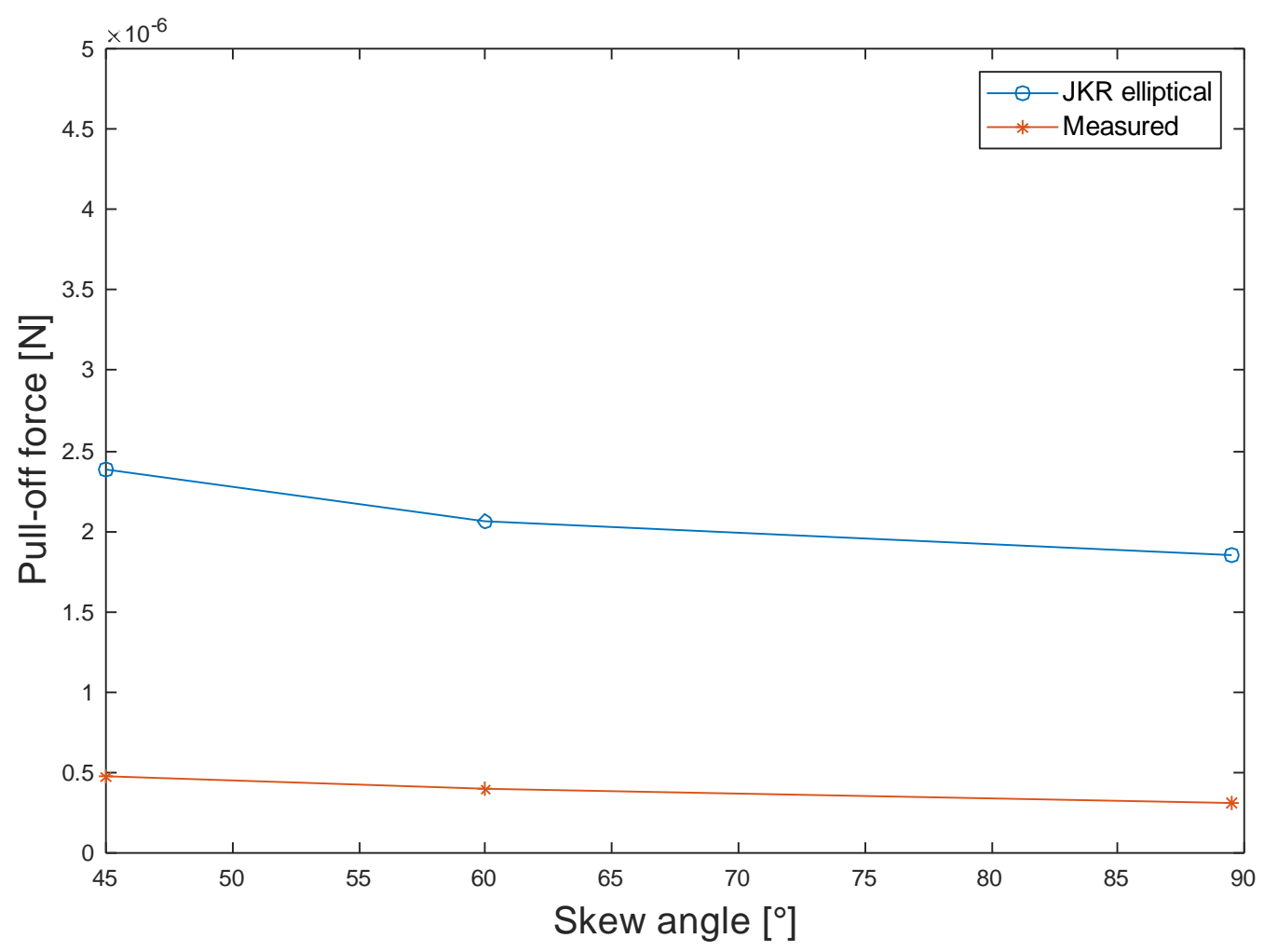

Fig. 4.2: Pull-off force comparison between the experimental results from [23] and the selected JKR elliptical model [15].

\subsection{Conclusions}

An adhesion map for elliptical contacts has been developed. It is shown that the limit between the JKR and the DH domain shifts closer to the DMT domain which becomes significant as the skew angles decreases. The constructed adhesion map can be used as a guide to select the suitable contact model for a specific elliptical contact condition. 



\section{Chapter 5}

\section{ADHESIVE NANOFIBER CONTACTS IN ELECTROSPUN NANOYARNS}

In this chapter, nanofiber contacts in electrospun nanoyarns are investigated to determine the significance of the presence of adhesion in such contacts. Contact and load properties are obtained using the geometrical model of an existing application with similar process. Adhesion is then modeled using the adhesive model that is relevant based on the adhesion map in Chapter 4.

\subsection{Introduction}

Using the model developed in Chapter 2 and Chapter 3, an adhesion map for elliptical contacts has been developed, see Chapter 4 . It is shown that adhesive elliptical contacts in general are dominated by the JKR type of adhesion [15], also supported by the findings in Chapter 3, which show that the limit between the JKR model and the DH model from Paper C of Part II is lower than $\mu=5$, for all $\theta_{\text {skew. Therefore, here the JKR elliptical model }}$ has been chosen to analyse the significance of the presence of adhesion in fiber-based applications such as nanofiber contacts in electrospun nanoyarns.

Nanofibers are fibers that have diameters in the nanometer range, and a ratio of length to diameter of 100:1 [26]. Nanofibers can be produced from various polymers; this results in nanofibers having different physical properties and application potential. A typical method to produce nanofibers is electrospinning.

Electrospinning is a straightforward yarn production system that utilizes electric force to draw charged threads of polymer solutions to fiber diameters up to hundred nanometers [26]. To do this, polymer solution is forced through a syringe; a solution drop is then formed at the needle tip. A high voltage is applied to the needle, which induces electric charges within the fluid. When the applied voltage is greater than a critical voltage, the repulsive force within the charged solution is greater than its surface tension and a jet erupts from the tip of the needle. As this jet travels through the air, the solvent evaporates, and consequently a polymer fiber is gathered by a funnel-shaped collector, which functions as a negative electrode $[27,28]$. The collector rotates and exerts a twist on the nanofiber bundles to form a nanoyarn; the nanoyarn is then wound on a yarn rolling device or wrapped around another yarn as a protection. The electrospinning process is simplified graphically in Fig. 5.1.

Contact studies on nanofibers are normally done for contacts between single nanofibers, as seen in [29-31]. No existing research has been done to analyse the 
significance of the presence of adhesion at the nanofiber level within a nanoyarn. This chapter investigates the significance of the presence of adhesion in nanofiber contacts within electrospun nanoyarns, based on the ratio of the adhesive force and the total applied load. In the analysis, the effects of the yarn structure and the manufacturing properties are also investigated.

\subsection{Modelling adhesive nanofiber contacts in electrospun nanoyarns}

The nanofiber electrospinning process is similar to the manufacturing process of a Stranded Wire Helical Spring (SWHS). SWHS is a unique helical spring, normally made up of several steel wires that are tied together to form a multilayer and coaxial strand, which is then twisted to form a helical spring, with the same direction as the spiral [32]. Typically, it is assumed that the wires in the cable of SWHS have frictionless contact between them. Based on the results in [33], the contacts between stranded wires inside the cable are elliptical contacts, rather than line contacts. This becomes the foundation of the SWHS model, modelling the elliptical contacts between the stranded wires in the cable. When compared to the nanofibers electrospinning process, stranded wires are similar to the nanofibers while the cable is similar to the nanoyarns. The translation of the nanofiber electrospinning process to the SHWS geometrical model is illustrated graphically in Fig. 5.1(a) and Fig. 5.1(b).

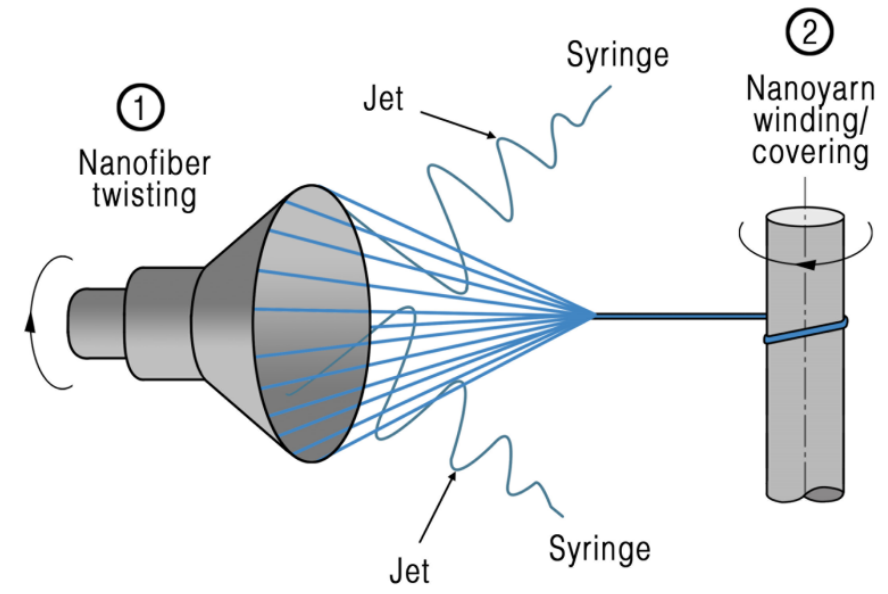

(a)

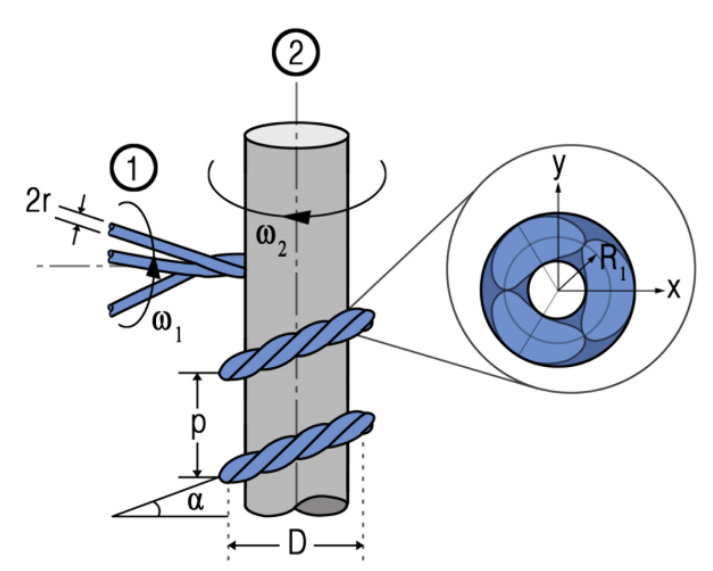

(b)

Fig 5.1: (a) Nanoyarn electrospinning process (b) SWHS geometrical representation for the nanoyarn electrospinning process. 
The skew angle between adjacent nanofibers in the protective yarn $\theta_{\text {skew }}$ is given in [33] as:

$$
\cos \theta_{\text {skew }}=\frac{\vec{a}_{1} \cdot \vec{a}_{2}}{\left|\vec{a}_{1}\right| \cdot\left|\vec{a}_{2}\right|}
$$

where $\vec{a}_{1}$ and $\vec{a}_{2}$ are the tangential vectors in any point of the central line of two adjacent nanofibers. Based from the analysis in [32], the general normal contact force value, $W_{f}$ between any two nanofibers is given as:

$$
W_{f}=W_{y} \cos \alpha /(n-1)
$$

where $W_{y}$ is the axial load applied to the structure, $\alpha$ is the nanoyarn wrapped angle and $n$ is the number of nanofibers in a nanoyarn.

Here, the JKR elliptical model in [15] is used to model adhesion between the nanofibers in electrospun nanoyarns. When two nanofibers are brought into contact, the normal load is given as:

$$
W_{J K R}=2 \pi a b\left[p_{1}-\left(\Delta_{1} a^{2}+\Delta_{2} b^{2}\right) / 3\right]
$$

which in this chapter is determined at the pull-off moment. Solutions for $a, b, p_{1}, \Delta_{1}, \Delta_{2}$ and corresponding equations can be found in Paper D, Part II.

\subsection{Results and discussion}

The presence of adhesion between nanofibers in electrospun nanoyarns is investigated on nylon 6,6 nanofibers. Table 5.1 lists the material properties while Table 5.2 lists the electrospinning properties. The chosen applied load is $10 \%$ from the breaking load of nylon 6 nanofibers that have similar properties as nylon 6,6 obtained from [34]. Parameters that affect adhesion in the nanofiber contacts using the values in Table 5.1 and Table 5.2 are taken as the reference properties. These chosen values are analysed to see if any changes in these values will affect adhesion between adjacent nanofibers in electrospun nanoyarns. The steps to obtain the results are explained in Paper D, Part II. 
Table 5.1: Properties of the chosen nanofiber.

\begin{tabular}{|l|c|c|c|}
\hline Properties & Nylon 6,6 & Unit & Ref. \\
\hline Transverse elastic modulus, $E_{\text {trans }}$ & 3 & $\mathrm{Gpa}$ & {$[35]$} \\
\hline Poisson's ratio, $\vartheta$ & 0.39 & - & {$[35]$} \\
\hline Work of adhesion & 46.5 & $\mathrm{~mJ} / \mathrm{m}^{2}$ & {$[35]$} \\
\hline Interatomic spacing, $z_{0}$ & 4.4 & $\AA$ & {$[35]$} \\
\hline
\end{tabular}

Table 5.2: Properties of the electrospinning process.

\begin{tabular}{|l|c|c|c|}
\hline Properties & Value & Unit & Ref. \\
\hline Helix angle, $\alpha$ & $\pi / 4$ & $\mathrm{rad}$ & \\
\hline Inner diameter, $D_{\text {core }}$ & 2 & $\mathrm{~mm}$ & \\
\hline Applied load, $W$ & 15.2 & $\mu \mathrm{N}$ & {$[34]$} \\
\hline Nanofiber radius, $r$ & 800 & $\mathrm{~nm}$ & {$[36]$} \\
\hline Twisting speed, $\omega_{1}$ & $2 \pi / 3$ & $\mathrm{rad} / \mathrm{s}$ & {$[28]$} \\
\hline Reeling speed, $\omega_{2}$ & $\pi / 6$ & $\mathrm{rad} / \mathrm{s}$ & {$[28]$} \\
\hline
\end{tabular}

Figure 5.2 shows the effect of nanofiber radius on the adhesive contacts and also the effect of the number of nanofibers in nanoyarns. The larger nanofiber radius does not affect the $\theta_{\text {skew }}$ values regardless of the nanofiber radius. However, the larger nanofiber radius is shown to result in higher $\mu$, larger contact area and greater pull-off force. For materials in the JKR domain, the resulting contact deformation is larger and the pull-off force is lower than for the less elastic materials. Yet, for nanoyarns that consist of many highly elastic nanofibers the space between nanofibers is limited, hence higher pull-off force is needed to separate the nanofibers in contact. It should be noted that for both fiber radius cases at $n=7$ the contact area and the pull-off force cannot be predicted as the $\theta_{\text {skew }}$ values are too low for the JKR elliptical contact. In general, a larger number of

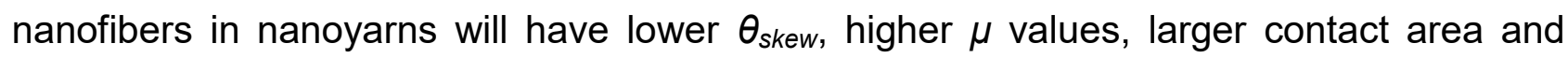
greater pull-off force.

In terms of the effect of higher applied load, there is no change in the adhesive contacts apart than affecting the load between nanofibers in contact; change in load results only in a larger Hertzian contact area. Being independent of the applied load is a characteristic of a JKR contact. 

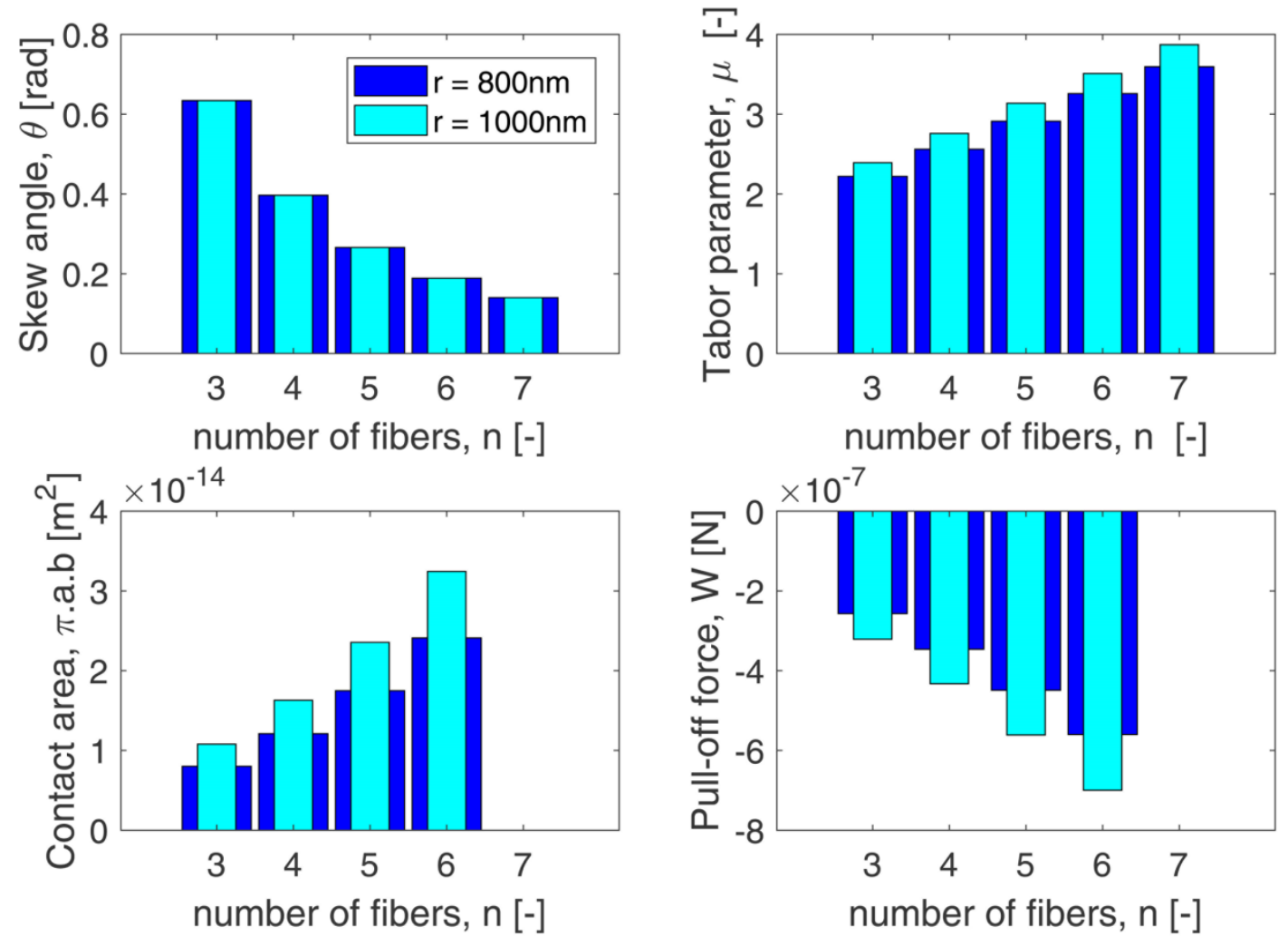

Fig. 5.2: The effect of number of fibers in a nanoyarn and nanofiber radius on (a) angle between the fibers, $\theta_{\text {skew }}$ (b) Tabor parameter, $\mu$, (c) contact area, and (d) pull-off force, $W$.

The effect of a higher nanofiber twisting speed $\omega_{1}$ of $5 \pi / 6$ and higher nanofiber reeling speed of $\pi / 3$ are shown in Fig. 5.3 and Fig. 5.4 respectively. Results show that the effects of $\omega_{1}$ are opposite to those of $\omega_{2}$. Higher $\omega_{1}$ is shown to result in higher $\theta_{\text {skew, }}$ lower $\mu$ values, smaller contact area and lower pull-off force. Higher $\omega_{1}$ results in more nanofiber sections to be twisted, hence it increases the $\theta_{\text {skew }}$ between adjacent nanofibers. At $n=7$ for higher $\omega_{1}$ and at $n>3$ for higher $\omega_{2}$, the contact area and the pull-off force cannot be predicted as the $\theta_{\text {skew }}$ is too low for the JKR elliptical contact, similar to the situation for $n=$ 7 of previous results in Fig. 5.2. 

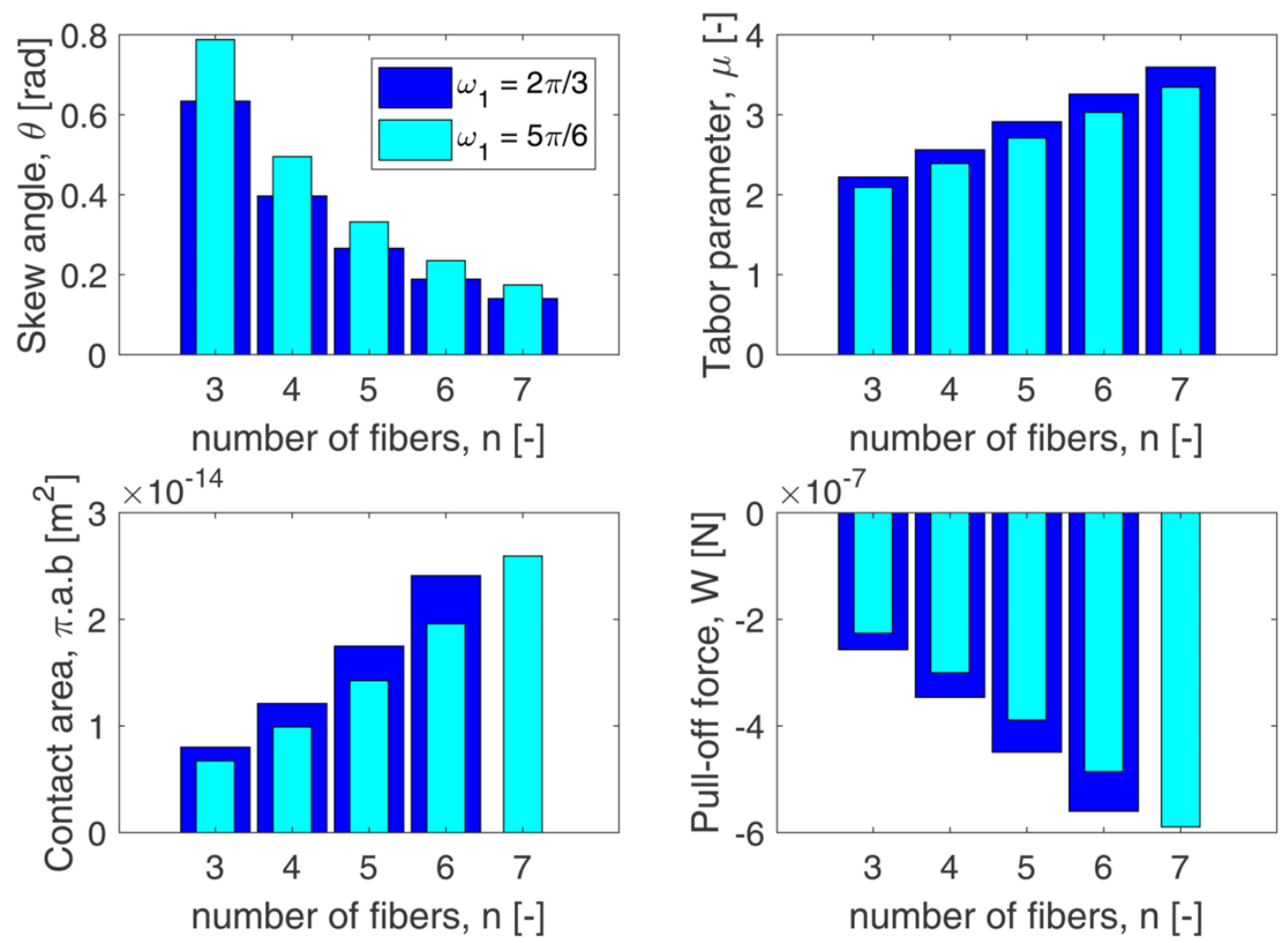

Fig. 5.3: The effect of nanofiber twisting speed on (a) angle between the fibers, $\theta_{\text {skew, }}$ (b) Tabor parameter, $\mu$, (c) contact area, and (d) pull-off force, $W$. 

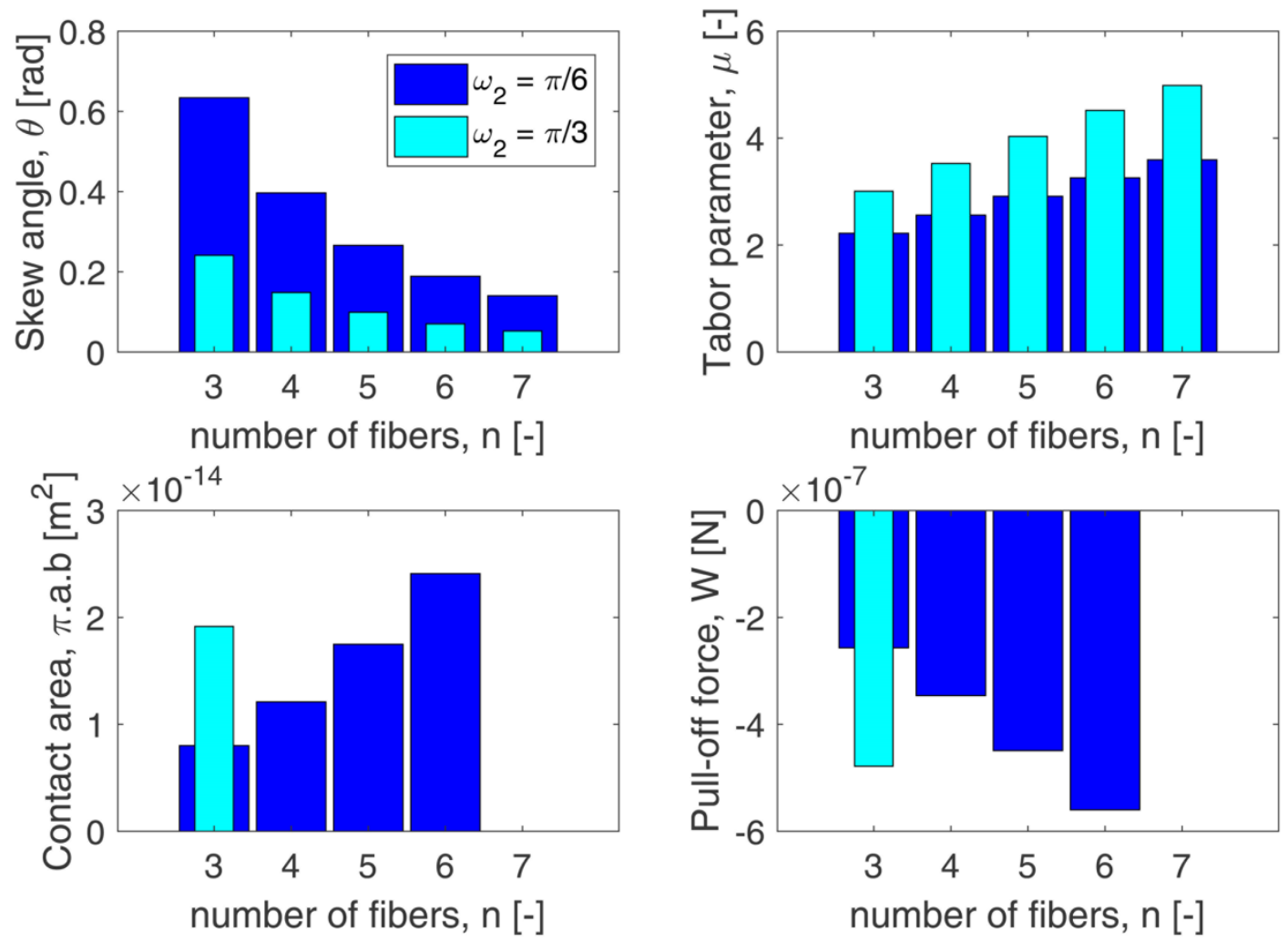

Fig. 5.4: The effect of reeling speed on (a) angle between the fibers, $\theta_{\text {skew, }}$ (b) Tabor parameter, $\mu$, (c) contact area, and (d) pull-off force, $W$.

Obviously, the adhesive force can be neglected at high load, as the adhesive load is smaller than the applied load. In this case, the contact can be regarded as a non-adhesive contact. Following the limit set in Chapter 4 to differentiate between adhesive contacts and non-adhesive contacts, the significance of the presence of adhesion in the contact between nanofibers in electrospun nanoyarns is determined using the ratio of the pull-off force to the total load, given as:

$$
\left|\frac{W_{J K R}}{\text { Wtotal }}\right|>0.05
$$

Setting the minimum limit to 0.05 , it means that the contact is regarded as adhesive when the pull-off force is more than five percent of the total load. $W_{\text {total }}$ is given as:

$$
W_{\text {total }}=W_{J K R}+W_{f}
$$


Fig. 5.5 shows the result of the ratio between $W_{J K R} / W_{\text {total }}$ for all the analysed parameters. The majority of the contacts have ratios higher than 0.05 , indicating that adhesion is indeed very significant in those contacts. However, as the number of nanofibers increases, the nanoyarn becomes packed; the nanofibers are almost parallel to each other and contacts between nanofibers have really low $\theta_{\text {skew }}$ values. Hence, the JKR model cannot predict the adhesive behaviour of these contacts.

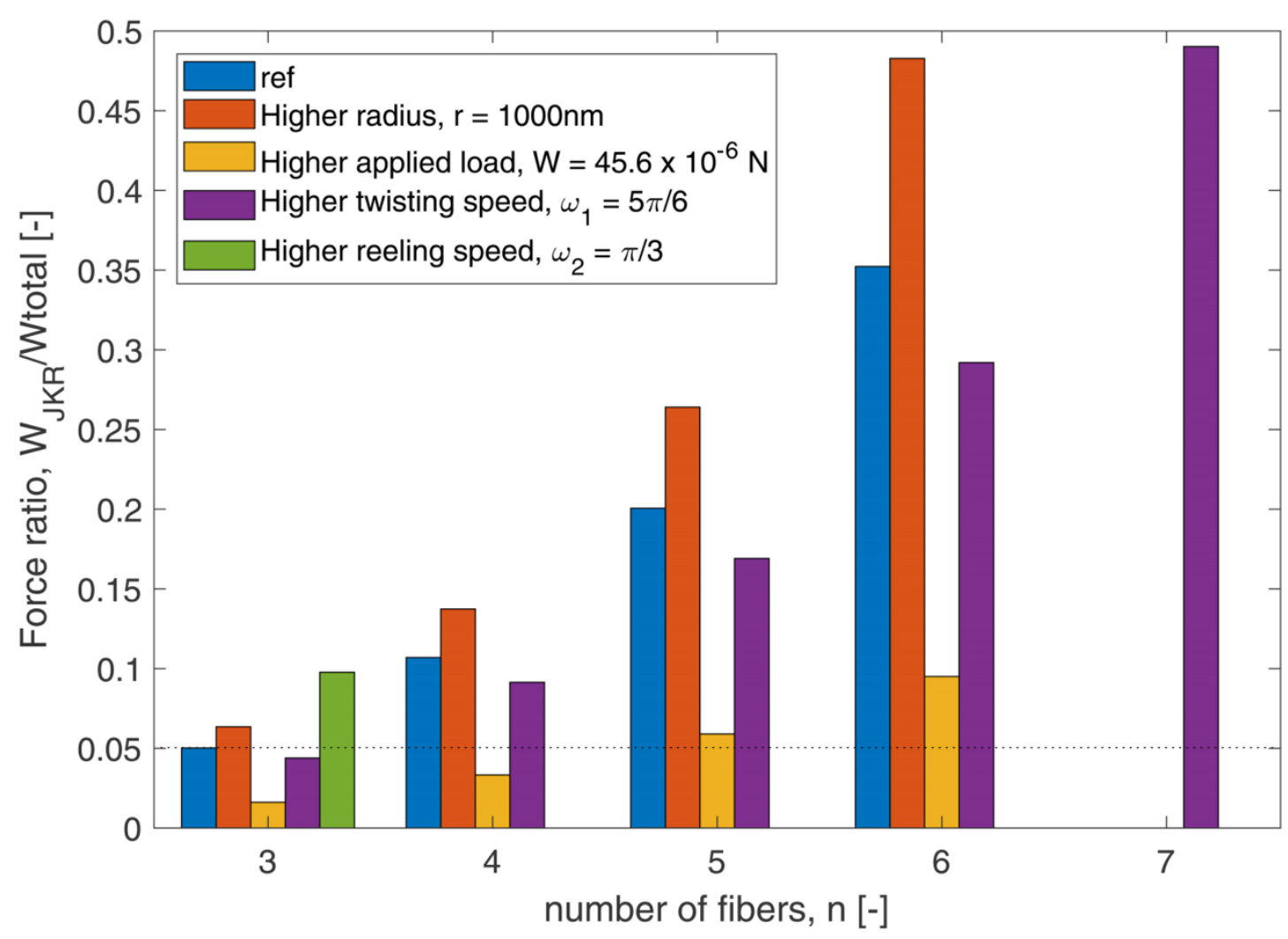

Fig 5.5: The force ratio for all parameters.

\subsection{Conclusion}

The significance of the presence of adhesion between nanofibers in nanoyarns is investigated in this paper. Load and contact properties of the nanofiber contacts in electrospun nanoyarns are obtained using the SWHS geometrical model, which then become the input for the JKR elliptical model. The significance of the presence of adhesion in nanofiber contacts is shown by the ratio of adhesive forces to the total load; properties such as nanofiber radius, the number of nanofibers in a nanoyarn, nanofiber twisting speed and nanofiber reeling speed are shown to influence adhesion at the nanofiber level. It has been shown that in most cases adhesion between nanofibers in electrospun nanoyarns cannot be ignored. 


\section{Chapter 6 \\ DISCUSSION AND CONCLUSION}

From the work described in the previous chapters, the significance of the presence of adhesion in real applications and the practicality of the developed DH model compared to the JKR elliptical model are discussed in this chapter. General conclusions are drawn from the research and recommendations for future work are suggested.

\subsection{Discussion}

\section{Adhesion in real application}

The contact behaviour at the fiber level is typically influenced by interface bonds such as adhesion; hence for single fiber-on-fiber contacts it is important to include adhesion in the contact mechanics models, as seen in modelling approaches [7,9,11-12,15,18] and experiments $[16,23]$. If adhesion is significant at fiber level, adhesive contact areas will be larger than non-adhesive contact areas and surface separation can be made only by exerting a pull-off force.

In real applications, adhesion is often disregarded as a way to simplify the contact of the already complicated structures. For ropes, it is generally assumed that the contacts between fibers are always line contacts; adhesion is shown to have minor influence in these contacts. However, based on the work done in Chapter 5 for nanoscale materials, adhesion cannot be disregarded. The results show that the presence of adhesion is significant between the nanofiber contacts in electrospun nanoyarns. When adhesion is present between nanofibers, adjacent nanofibers are bonded together with a contact area that is larger than for non-adhesive contacts. For single nanofiber contacts, both nanofibers are separated more easily than nanofibers in a nanoyarn: the spaces between nanofibers in a nanoyarn are limited, thus bonded nanofibers will continue to be in contact. The enlarged contact area due to adhesion will also increase friction between nanofibers, which can be undesirable depending on the applications. To summarize, adhesion has been shown to be significant only at the nanoscale level while it can be ignored for contacts at the microscale level. 


\section{Adhesive elliptical contacts: JKR model vs DH model}

For circular contacts, the adhesion map in [13] lays out clearly the validity domain for each of the adhesive models. The $\mathrm{DH}$ and the MD models are shown to be dominant for a large portion of the adhesion map, within the range of $0.1 \leq \mu \leq 5$. As for the elliptical contact, the numerical results in Paper $\mathrm{C}$ of Part II show that the pull-off force predicted by the JKR elliptical model approaches the pull-off force of the $\mathrm{DH}$ elliptical model at $\mu$ much less than 5 , which is a typical value for circular contacts.

As the skew angle between the cylindrical bodies decreases, the limit between the $\mathrm{DH}$ and the JKR models moves to the left in the adhesion map for elliptical contacts, see Fig. 4.1. This means that as the contact gets further away from a circular contact, the application domain for the DH elliptical model becomes smaller while the JKR domain becomes bigger. In general, the JKR elliptical model is more practical for modelling general adhesive elliptical contacts. Nevertheless, the DH model remains relevant for adhesive elliptical contacts, particularly for fibers with smaller radii.

\subsection{Conclusion}

The purpose of this thesis is to provide an insight into the contact mechanics of the fiberon-fiber contacts. The mechanics of fiber-on-fiber contacts was studied, with a focus on modelling an adhesive elliptical contact. In Chapter 2, the development of the contact model for adhesive elliptical contacts was described, which was shown to be suitable only for nearly circular contacts as the exact geometry of the elliptical contact and the corresponding adhesive zone are unknown a priori. The work was continued in Chapter 3 , where geometrical equations for the annular adhesive region were obtained, to allow the developed adhesive model to predict the pull-off force accurately. Following the circular contacts, an adhesion map was developed in Chapter 4 to guide the selection of currently available adhesive elliptical models. This adhesion map provides the application limit for each adhesive elliptical model. Finally, in Chapter 5, adhesive nanofiber contacts in electrospun nanoyarns were investigated using the relevant adhesive model selected from the adhesion map in Chapter 4, to see the significance of the presence of adhesion in such contacts.

Overall, the work done in this thesis can be summarized as follows:

1. The fiber-on-fiber contact between cylindrical bodies for various contact conditions is modelled based on the $\mathrm{DH}$ theory.

2. Geometrical equations for the ellipticity ratio of both contact and adhesive ellipses and for the semi-major axis of the contact ellipse have been obtained. This enables 
the developed $\mathrm{DH}$ elliptical contact model to be applied for both nearly circular and elliptical contacts.

3. An adhesion map to guide the adhesive model selection for adhesive elliptical contacts has been developed.

4. A relevant adhesive model based on the developed adhesion map for elliptical contacts has been applied to model the nanofiber contacts in electrospun nanoyarns, showing that adhesion is indeed significant in the contacts at nanofiber level.

\subsection{Recommendations for future research}

The DH model developed in Chapter 2 shows the contact mechanics for adhesive elliptical contacts; it is learnt that the inner and the outer boundaries of the adhesive region are load-dependent. With this new knowledge, geometry equations for the adhesive region were fitted in Chapter 3, allowing the developed $\mathrm{DH}$ model to be used for various elliptical contact conditions. The developed $\mathrm{DH}$ model provides the foundation to further understand adhesive elliptical contacts. Several recommendations are presented below for future work to improve the model.

\section{Obtain the contact angle limit of the DH elliptical model}

Currently, there is no known validity limit with respect to the $\theta_{\text {skew }}$ for the developed model. For accurate representation of the contact, the angle limit of the $\mathrm{DH}$ elliptical model can be found by comparing it with experimental and numerical data, similar to the comparison made for the JKR-based contacts. A transition function then can be derived between the angle limit and zero degree (angle representing the line contacts) to allow adhesive contact modelling at very low $\theta_{\text {skew }}$ values.

\section{Adhesive contact modelling for fiber contacts in complex structures}

Contacts in complex structures such as ropes occur at different length scales. Contact modelling of such structures can utilise the SWHS geometrical model that is established to model more than a single material layer. The inner layer (modelling level for Chapter 5), the middle layer and the outer layer of the structure, such as shown in Fig. 6.1, can be modelled using the SHWS geometrical model. 


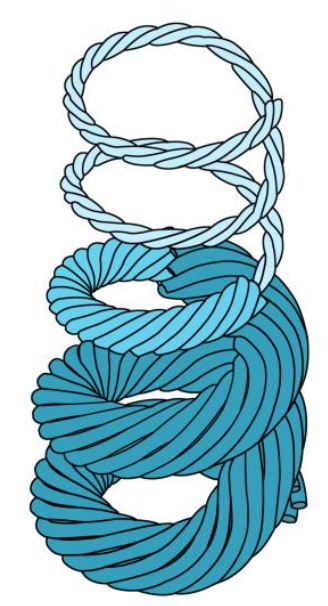

Fig. 6.1: Possible fiber contact modelling for different layer of materials using the SWHS geometrical model.

\section{Collapse analysis of nanofibers}

When a fiber network is subjected to any external disturbance, fibers in the network may deflect and stick to adjacent fibers due to adhesion and the tendency of fibers to bending. Fiber collapse due to adhesion has been known to reduce the performance of nanofiber networks that can result in nonlinear behaviour. Collapse analysis of nanofibers can be done by developing a fiber collapse model as in [37] to analyse the effects of fiber elasticity, surface adhesion and fiber geometries on the critical collapse distance. 


\section{References}

[1] E. Kvavadze, O. Bar-Yosef, A. Belfer-Cohen, E. Boaretto, N. Jakeli, Z. Matskevich and T. Meshveliani, "30,000-Year-Old Wild Flax Fibers", Science, vol. 325, no. 5946, pp. 1359-1359, 2009.

[2] T. Hussain, M. Tausif and M. Ashraf, "A review of progress in the dyeing of ecofriendly aliphatic polyester-based polylactic acid fabrics", Journal of Cleaner Production, vol. 108, pp. 476-483, 2015.

[3] P. Lord, Handbook of yarn technology. Boca Raton, Fla.: CRC, 2003.

[4] Y. Wu, D. U. Shah, C. Y. Liu, Z. Y. Yu, J. Liu, X. H. Ren, M. J. Rowland, C. Abell, M. H. Ramage and O. A. Scherman, "Bioinspired supramolecular fibers drawn from a multiphase self-assembled hydrogel", Proceedings of the National Academy of Sciences, USA, vol. 114, no. 31, pp.8163-8168, 2017.

[5] H. Hertz, "Über die Berührung fester elastischer Körper", J. reine und angewandte Mathematik, vol. 92, pp. 156-71, 1882.

[6] N. Rodríguez Pareja, "Contact and Friction in Systems with Fibre Reinforced Elastomers", Ph.D thesis, University of Twente, 2012.

[7] K. L. Johnson, K. Kendall and A. D. Roberts, "Surface energy and the contact of elastic solids", Proceedings of the Royal Society of London. Series A, Mathematical and Physical Sciences, vol. 324, pp. 301-313, 1971.

[8] K. Kendall, "Adhesion: Molecules and Mechanics", Science, vol. 263, no. 5154, pp. 1720-1725, 1994.

[9] B. Derjaguin, V. Muller and Y. Toporov, "Effect of contact deformations on the adhesion of particles", Journal of Colloid and Interface Science, vol. 53, no. 2, pp. 314-326, 1975.

[10] D. Tabor, "Surface forces and surface interactions", Journal of Colloid and Interface Science, vol. 58, no. 1, pp. 2-13, 1977.

[11] D. Maugis, "Adhesion of spheres: The JKR-DMT transition using a Dugdale model", Journal of Colloid and Interface Science, vol. 150, no. 1, pp. 243-269, 1992.

[12] J. A. Greenwood and K. L. Johnson, "An alternative to the Maugis model of adhesion between elastic spheres", Journal of Physics D: Applied Physics, vol. 31, no. 22, pp. 3279-3290, 1998.

[13] K. L. Johnson and J. A. Greenwood, "An Adhesion Map for the Contact of Elastic Spheres", Journal of Colloid and Interface Science, vol. 192, no. 2, pp. 326-333, 1997. 
[14] Y. Zhang, "Transitions between different contact models", Journal of adhesion science and Technology, vol. 22, no. 7, pp. 699-715, 2012.

[15] K. L. Johnson and J. A. Greenwood, "An approximate JKR theory for elliptical contacts", Journal of Physics D: Applied Physics, vol. 38, no. 7, pp. 1042-1046, 2005.

[16] B. Sümer, C. Onal, B. Aksak and M. Sitti, "An experimental analysis of elliptical adhesive contact", Journal of Applied Physics, vol. 107, no. 11, p. 113512, 2010.

[17] C. Jin, A. Jagota and C. Hui, "An easy-to-implement numerical simulation method for adhesive contact problems involving asymmetric adhesive contact", Journal of Physics D: Applied Physics, vol. 44, no. 40, p. 405303, 2011.

[18] X. Wu and Y. Dzenis, "Adhesive contact in filaments", Journal of Physics D: Applied Physics, vol. 40, no. 14, pp. 4276-4280, 2007.

[19] R. Bradley, "The cohesive force between solid surfaces and the surface energy of solids", The London, Edinburgh, and Dublin Philosophical Magazine and Journal of Science, vol. 13, no. 86, pp. 853-862, 1932.

[20] B. Cornelissen, "The role of friction in tow mechanics", Ph.D thesis, University of Twente, 2018.

[21] K. L. Johnson, Contact mechanics. Cambridge: Cambridge University Press, 2004.

[22] M. Bazrafshan, M. B. de Rooij, M. Valefi and D. J. Schipper, "Numerical method for the adhesive normal contact analysis based on a Dugdale approximation", Tribology International, vol. 112, pp. 117-128, 2017.

[23] N. Ismail, H. Gojzewski, M. B. de Rooij, D. J. Schipper and G. J. Vancso, "Adhesion force measurement between single aramid fibres", unpublished.

[24] S. Spells, Characterization of solid polymers, $1^{\text {st }}$ ed. London: Chapman \& Hall, 1994.

[25] P. van Zwol, G. Palasantzas and J. De Hosson, "Influence of random roughness on the adhesion between metal surfaces due to capillary condensation", Applied Physics Letters, vol. 91, no. 10, p. 101905, 2007.

[26] U. Ali, Y. Zhou, X. Wang and T. Lin, "Electrospinning of Continuous Nanofiber Bundles and Twisted Nanofiber Yarns", in Nanofibers-production, properties and functional applications, T. Lin, Ed. InTech, 2011, pp. 153-174.

[27] A. Hamed, N. Shehata and M. Elosairy, "Investigation of Conical Spinneret in Generating More Dense and Compact Electrospun Nanofibers", Polymers, vol. 10, no. 1, p. 12, 2017. 
[28] S. Jin, B. Xin and Y. Zheng, "Preparation and Characterization of Polysulfone Amide Nanoyarns by the Dynamic Rotating Electrospinning Method". Textile Research Journal, 0(00), 1-11, 2017.

[29] Q. Shi, K. Wan, S. Wong, P. Chen and T. Blackledge, "Do Electrospun Polymer Fibers Stick?", Langmuir, vol. 26, no. 17, pp. 14188-14193, 2010.

[30] M. Xing, W. Zhong, X. Xu and D. Thomson, "Adhesion Force Studies of Nanofibers and Nanoparticles", Langmuir, vol. 26, no. 14, pp. 11809-11814, 2010.

[31] Q. Shi, S. Wong, W. Ye, J. Hou, J. Zhao and J. Yin, "Mechanism of Adhesion between Polymer Fibers at Nanoscale Contacts", Langmuir, vol. 28, no. 10, pp. 4663-4671, 2012.

[32] S. Wang, X. Li, S. Lei, J. Zhou and Y. Yang, "Research on torsional fretting wear behaviors and damage mechanisms of stranded-wire helical spring", Journal of Mechanical Science and Technology, vol. 25, no. 8, pp. 2137-2147, 2011.

[33] X. Li, S. Wang and J. Zhou, "Analysis of elliptical Hertz contact of steel wires of stranded-wire helical spring", Journal of Mechanical Science and Technology, vol. 28, no. 7, pp. 2797-2806, 2014.

[34] M. Bazbouz and G. Stylios, "The tensile properties of electrospun nylon 6 single nanofibers", Journal of Polymer Science Part B: Polymer Physics, vol. 48, no. 15, pp. 1719-1731, 2010.

[35] W. Callister and D. Rethwisch, Fundamentals of material science and engineering: an integrated approach., 3rd ed. John Wiley \& Sons, Inc, 2008.

[36] T. A. Skotheim and J. Reynolds, Conjugated polymers: processing and applications, CRC Press, 2006.

[37] X. Wu and Y. A. Dzenis, "Collapse analysis of nanofibers", Nanotechnology, vol. 18, pp. 1-6, 2007. 

Part II 



\section{Part II}

Paper A. N. H. M. Zini, M. B. de Rooij, M. Bazr Afshan Fadafan, N. Ismail and D. J. Schipper, "Extending the Double-Hertz Model to Allow Modeling of an Adhesive Elliptical Contact", Tribology Letters, vol. 66, no. 1, 2018.

Paper B. N. H. M Zini, M. B. de Rooij, M. Bazr Afshan Fadafan, N. Ismail and D. J. Schipper, "Response to Greenwood's Comment on "Extending the Double-Hertz Model to Allow Modeling of an Adhesive Elliptical Contact" ", Tribology Letters, vol. 66, no. 3, 2018.

Paper C. N. H. M. Zini, M. B. de Rooij, M. Bazr Afshan Fadafan, A. Akchurin and D. J. Schipper, "Predicting the Pull-off Force of the Double-Hertz Elliptical Contact With a Loaddependent Adhesive Region", submitted to Tribology Transactions, 2018.

Paper D. N. H. M. Zini, M. B. de Rooij and D. J. Schipper, "Adhesive Nanofiber Contacts in Electrospun Nanoyarns", submitted to Textile Research Journal, 2018. 



\title{
Paper A
}

\section{Extending the double-Hertz model to allow modeling of an adhesive elliptical contact}

\author{
N.H.M. Zini ${ }^{1,2,{ }^{*}, \text { M.B. de Rooij }}{ }^{1}$, M. Bazrafshan ${ }^{1}$, N. Ismail ${ }^{1,2}$, D.J. Schipper ${ }^{1}$ \\ ${ }^{1}$ Department of Surface Technology and Tribology, Faculty of Engineering Technology, \\ University of Twente, the Netherlands \\ ${ }^{2}$ Faculty of Mechanical Engineering, Universiti Teknikal Malaysia Melaka, Hang Tuah \\ Jaya, 76100 Durian Tunggal, Melaka, Malaysia
}

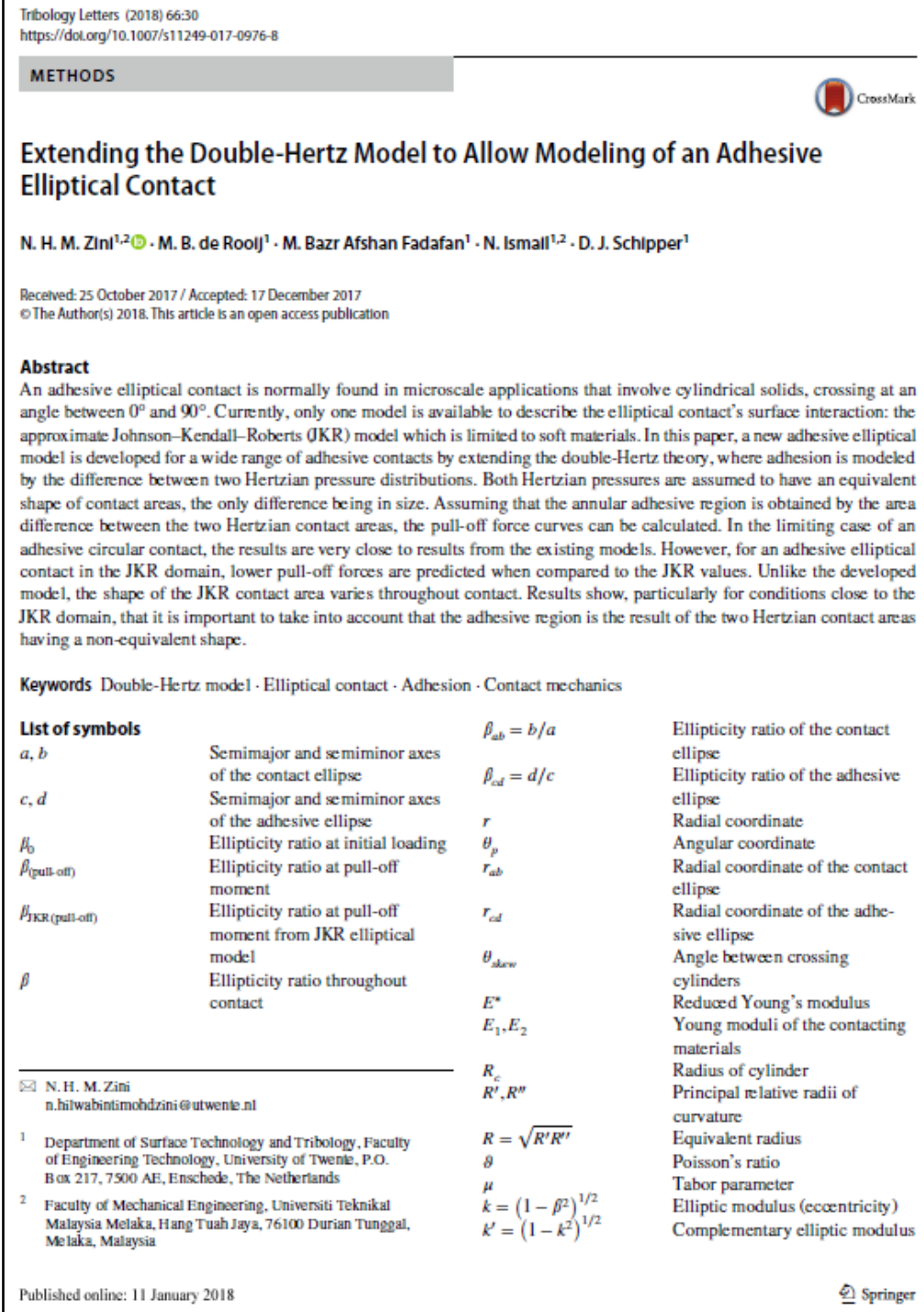

Extending the Double-Hertz Model to Allow Modeling of an Adhesive Elliptical Contact

N. H. M. ZIn| $\left.\right|^{1,2} \oplus \cdot$ M. B. de Roolj $~ \cdot$ M. Bazr Afshan Fadafan' $\cdot$ N. Ismall ${ }^{1,2} \cdot$ D. J. Schipper ${ }^{1}$

Recelved: 25 October 2017/ Accepted: 17 December 2017

oThe Author(s) 2018. This article is an open access publication

Abstract

An adhesive elliptical contact is normally found in microscale applications that involve cylindrical solids, crossing at an angle between $0^{\circ}$ and $90^{\circ}$. Currently, only one model is available to describe the elliptical contact's surface interaction: the approximate Johnson-Kendall-Roberts (JKR) model which is limited to soft materials. In this paper, a new adhesive elliptical model is developed for a wide range of adhesive contacts by extending the double-Hertz theory, where adhesion is modeled by the difference between two Hertzian pressure distributions. Both Hertzian pressures are assumed to have an equivalent shape of contact areas, the only difference being in size. Assuming that the annular adhesive region is obtained by the area difference between the two Hertian contact areas, the pull-off force curves can be calculated. In the limiting case of an adhesive circular contact, the results are very close to results from the existing models. However, for an adhesive elliptical contact in the JKR domain, lower pull-off forces are predicted when compared to the JKR values. Unlike the developed model, the shape of the JKR contact area varies throughout contact. Results show, particularly for conditions close to the JKR domain, that it is important to take into account that the adhesive region is the result of the two Hertzian contact areas having a non-equivalent shape.

Keywords Double-Hertz model · Elliptical contact · Adhesion · Contact mechanics

List of symbols

$a, b$

$c, d$

$B_{0} \quad$ Ellipticity ratio at initial loading

$\beta_{\text {(poul- off }} \quad$ Ellipticity ratio at pull-off

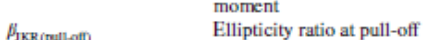
moment from JKR elliptical model

Ellipticity ratio throughout

contact

$\beta$

$\beta_{a b}=b / a$

$p_{c d}=d / c$

$\dot{\theta}_{p}$

$r_{a b}$

$r_{c d}$

$\theta_{\text {sem }}$

$E^{*}$
$E_{1}, E_{2}$

$R_{c}$
$R^{\prime}, R^{\prime \prime}$

N.H.M.Zini
n. hilwabintimohdrini utwenten

Department of Surface Technology and Tribology, Faculty of Enginoering Tectinoloyy, University of Twente, P.O.

Bax 217, 7500 AE, Enschede, The Netherlands

Faculty of Mechanical Engineering. Universiti Teknikal Malaysia Melaka, Hang Tuah Jaya, 76100 Durian Tung̨al, Melaka, Malays

Ellipticity ratio of the contact

ellipse

Ellipticity ratio of the adhesive

ellipse

Radial coordinate

Angular coordinate

Radial coordinate of the contact

ellipse

Radial coordinate of the adhe-

sive ellipse

Angle betwoen crossing

cylinders

Reduoed Young's modulus

Young moduli of the contacting

materials

Radius of cylinder

Principal relative radii of

curvature

Equivalent radius

Poisson's ratio

Tabor parameter

Elliptic modulus (ecoentricity)

Complementary elliptic modulus 

Abstract An adhesive elliptical contact is normally found in microscale applications that involve cylindrical solids, crossing at an angle between $0^{\circ}$ and $90^{\circ}$. Currently, only one model is available to describe the elliptical contact's surface interaction: the approximate Johnson-Kendall-Roberts (JKR) model which is limited to soft materials. Here, a new adhesive elliptical model is developed for a wide range of adhesive contacts by extending the double-Hertz theory, where adhesion is modeled by the difference between two Hertzian pressure distributions. Both Hertzian pressures are assumed to have an equivalent shape of contact areas, the only difference being in size. Assuming that the annular adhesive region is obtained by the area difference between the two Hertzian contact areas, the pull-off force curves can be calculated. In the limiting case of an adhesive circular contact, the results are very close to results from the existing models. However, for an adhesive elliptical contact in the JKR domain, lower pull-off forces are predicted when compared to the JKR values. Unlike the developed model, the shape of the JKR contact area varies throughout contact. Results show, particularly for conditions close to the JKR domain, that it is important to take into account that the adhesive region is the result of the two Hertzian contact areas having a non-equivalent shape.

Keywords double-Hertz model; elliptical contact; adhesion; contact mechanics

\section{List of symbols}

$a, b$

$c, d$

$\beta_{0}$

$\beta_{(p u l l-o f f)}$

$\beta_{J K R(p u l l-o f f)}$

$\beta$

$\beta_{a b}=b / a$

$\beta_{c d}=d / c$

$r$

$\theta_{p}$

$r_{a b}$

$r_{c d}$

$\theta_{\text {skew }}$

$E^{*}$
Semi-major and semi-minor axes of the contact ellipse

Semi-major and semi-minor axes of the adhesive ellipse

Ellipticity ratio at initial loading

Ellipticity ratio at pull-off moment

Ellipticity ratio at pull-off moment from JKR elliptical model

Ellipticity ratio throughout contact

Ellipticity ratio of the contact ellipse

Ellipticity ratio of the adhesive ellipse

Radial coordinate

Angular coordinate

Radial coordinate of the contact ellipse

Radial coordinate of the adhesive ellipse

Angle between crossing cylinders

Reduced Young's modulus 


$$
\begin{aligned}
& E_{1}, E_{2} \\
& R_{c} \\
& R^{\prime}, R^{\prime \prime} \\
& R=\sqrt{R^{\prime} R^{\prime \prime}} \\
& \vartheta \\
& \mu \\
& k=(1- \\
& k^{\prime}=(1 \\
& \varphi=\sin \\
& \boldsymbol{K}(k) \\
& \boldsymbol{E}(k) \\
& \boldsymbol{F}(\varphi, k) \\
& \boldsymbol{E}(\varphi, k) \\
& v \\
& W \\
& \Delta \gamma \\
& P o_{a b} \\
& P o_{c d} \\
& \sigma_{0} \\
& \omega_{i n_{a b}} \\
& \omega_{o u t_{a b}} \\
& \omega_{i n_{c d}} \\
& \omega_{0 a b} \\
& \omega_{a b c d} \\
& \delta \\
& z \\
& h \\
&
\end{aligned}
$$$$
R=\sqrt{R^{\prime} R^{\prime \prime}}
$$$$
k=\left(1-\beta^{2}\right)^{1 / 2}
$$$$
k^{\prime}=\left(1-k^{2}\right)^{1 / 2}
$$$$
\varphi=\sin ^{-1}\left(a / \sqrt{l+a^{2}}\right)
$$

Young moduli of the contacting materials

Radius of cylinder

Principal relative radii of curvature

Equivalent radius

Poisson's ratio

Tabor parameter

Elliptic modulus (eccentricity)

Complementary elliptic modulus

Second argument of the incomplete elliptic integrals

Complete elliptic integral of the first kind

Complete elliptic integral of the second kind Incomplete elliptic integral of the first kind Incomplete elliptic integral of the second kind Scaling factor to keep $\sigma_{0}$ and $\Delta y$ at a constant value Applied load of single asperity

Work of adhesion

Maximum pressure of the contact ellipse

Maximum pressure of the adhesive ellipse

Maximum adhesive stress

Surface displacement within the contact ellipse

Surface displacement outside the contact ellipse

Surface displacement within the adhesive ellipse

Combined surface displacement for $0 \leq r \leq r_{a b}$

Combined surface displacement for $r_{a b} \leq r \leq r_{c d}$

Approach of distant points

Initial gap in contact area

Separation between surfaces in the adhesive region

\section{Introduction}

Surface adhesion is important in the mechanics of surface contacts, in particular at microscale [1-4], as a result of high surface area-to-volume ratios [5]. Various models have been developed to describe the adhesive contact between surfaces. In the context of modeling smooth surfaces, three important adhesive contact theories exist: (1) the Johnson-Kendall-Roberts (JKR) model [6]; (2) the Derjaguin-Muller-Toporov (DMT) model 
[7], which was later corrected by Muller et al. [8]; and (3) the Maugis-Dugdale (MD) model [9]. In the case of a circular contact, the JKR model relies on the assumption that short range adhesion forces act within the contact area. Conversely, in the DMT model, long range adhesion forces are assumed to act outside the contact area. Later, it was found that the DMT model and the JKR model had different pull-off predictions. This debate continued until a finding by Tabor [10] revealed that both theories were actually complementary. Solid materials with low surface energy are particularly suited to using the DMT model, while the JKR model is suitable for soft materials with high surface energy values. The important criterion to distinguish between the DMT model and the JKR model is the neck formation outside the contact area. The Tabor parameter $\mu$ is used to measure the ratio of neck height to the equilibrium separation [10]. For high values of $\mu$, the adhesion forces outside the contact area can be disregarded as the surfaces are totally separated. This behavior perfectly describes the JKR contact [6]. As in the case of small $\mu$ values, the presence of adhesion forces is significant outside the contact area, as shown by the DMT model [7].

The transition between the DMT model and the JKR model is completed by the intermediate MD model that befits a wider range of common materials. Solutions from the MD model can be obtained analytically by solving a set of equations simultaneously. Greenwood and Johnson developed another transition model known as the double-Hertz (DH) model [11]. Not only has that model been proven to be feasible by producing similar results to those from the MD model, but it is also more straightforward in terms of mathematical formulations. All the models mentioned above have been extended for the application of line contacts, as shown by [12] with a JKR-based foundation and [13, 14] for cohesive MD models. The extension of the DH model was developed in [15]. Since its development, the $\mathrm{DH}$ model has been extended to various applications such as random multi-asperity contacts [16] and a sinusoidal wavy surface [17].

At present, there is only one contact model that can describe the mechanics of an adhesive elliptical contact, namely the approximate JKR model [18]. The model is built on the assumption that both major and minor axes have identical values of the stress intensity factor at the edges of the contact. This assumption is to avoid the separation at both ends of the major axis, while the stress intensity factor at both edges of the minor axis remains lower than the critical value. The shape of the elliptical contact area is shown to vary with the applied load. This behavior is different from that predicted by the Hertz theory in which the ellipse's growth rate remains radially constant as the load varies. Validations for the elliptical JKR model have been conducted by several researchers, either by experimental 
or numerical studies. A finding in [19] shows that the difference between the pull-off forces from the experimental data and the model becomes prominently greater as the contact area approaches a slim elliptical shape. The same result was also reported in numerical works in [20]. It is found that in contrast to the JKR elliptical theory, the pull-off forces decrease as the skew angles become smaller in the numerical simulations.

For circular contacts, prediction of the contact behavior in the adhesive region is straightforward with constant deformations throughout the periphery. In the case of elliptical contacts, it is clearly a complex contact, involving various contact geometries, ranging from nearly circular to slim elliptical contacts. The ellipticity ratio $\beta$ is introduced to illustrate the deviation of the ellipse from the circular shape, given as:

$$
\beta=\frac{\text { semi-minor axis }}{\text { semi-major axis }}
$$

From Eq. (1), the values of the ellipticity ratios are found to be within the range of $0<\beta<1$ where $\beta$ values closer to one have nearly circular contact areas, which are equivalent to having a nearly $90^{\circ}$ angle between the cylinders. $\beta$ values closer to zero have contact areas with shapes that resemble line forms, due to really small skew angles. The shape variations of an elliptical contact are shown schematically in Fig. 1.

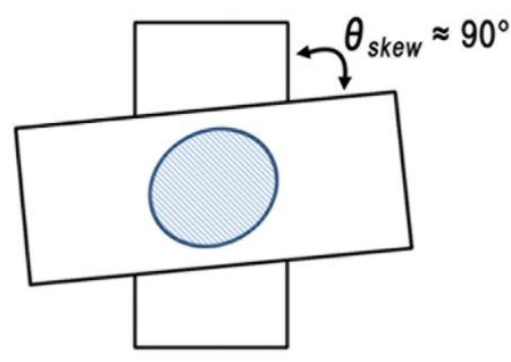

(a)

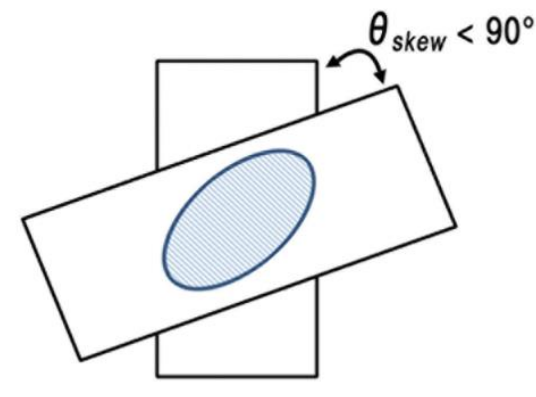

(b)

Fig. 1: Variation of an elliptical contact (a) Nearly circular contact for $\beta$ value close to 1 $\left(\theta_{\text {skew }} \approx 90^{\circ}\right)$ (b) Mildly elliptical contact for intermediate values of $\beta\left(\theta_{\text {skew }}<90^{\circ}\right)$.

The current work focuses on extending the $\mathrm{DH}$ model for adhesive elliptical contacts with high initial ellipticity ratios $\beta_{0}$ ranging from 0.8 to 0.99 . The developed model is expected to behave similarly to the current adhesive models in the limiting case of circular contacts within the range of $0.5 \leq \mu \leq 5$. In this paper, both contact and adhesive ellipses which bound the adhesive annular region are assumed to have identical, fixed ellipticity ratios throughout the contact, though the limit of this assumption must be evaluated. The developed model is also expected to follow the behavior of the JKR elliptical model in the JKR domain. It is shown that the pull-off behavior in the JKR domain is influenced by the unequal growth rate of its contact area in both major and minor axes 
directions. However, the question of whether the adhesive region of the developed model is also subjected to the unequal growth rate, inside and outside the JKR domain, needs to be investigated. These aspects are explored in this paper.

\section{Model development}

\subsection{Non-adhesive elastic contact}

The Hertz model, described in [21], was the pioneer of contact models. An elliptical contact

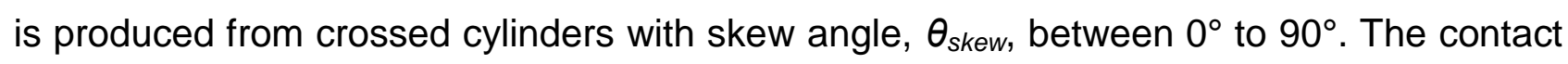
area is an ellipse, with semi-major axis $a$ and semi-minor axis $b$, as shown in Fig. 2.

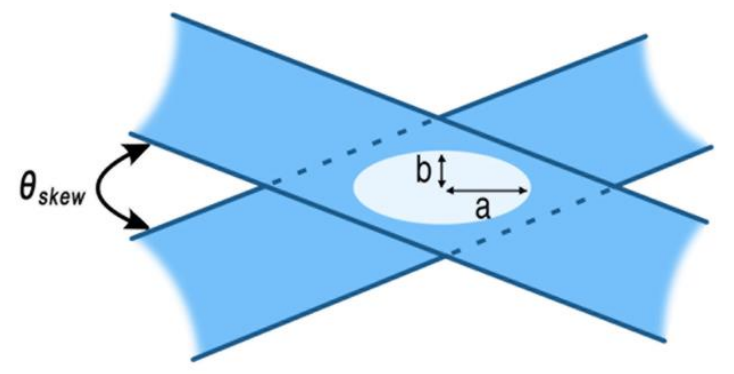

Fig. 2: An elliptical contact due to crossed cylinders $\left(0^{\circ}<\theta_{\text {skew }}<90^{\circ}\right)$.

Formulations for the non-adhesive elliptical contact model are given in [21], in Cartesian coordinates. In this paper, all the equations are expressed in a polar coordinate system, where geometrical parameters are defined in Appendix A.

The initial gap, $z$, in polar coordinates is given in [21] by the general expression:

$$
z\left(r, \theta_{p}\right)=\frac{1}{2 R^{\prime}}\left(r \cos \theta_{p}\right)^{2}+\frac{1}{2 R^{\prime \prime}}\left(r \sin \theta_{p}\right)^{2}
$$

where $R^{\prime}$ and $R^{\prime \prime}$ are the first and the second principal relative radii of curvature for the cylinders. $r$ is the radial coordinate and $\theta_{p}$ is the angular coordinate of the chosen point in the contact region. $R$ ' and $R$ " are related to the angle between the crossing cylinders, $\theta_{\text {skew, }}$ by:

$$
\begin{aligned}
& R^{\prime}=R_{c} /\left(1-\cos \theta_{\text {skew }}\right) \\
& R^{\prime \prime}=R_{c} /\left(1+\cos \theta_{\text {skew }}\right)
\end{aligned}
$$

where the cylinders in contact are assumed to have the same radius $R_{c}$. 
The effective radius, $R$, is given by:

$$
R=\sqrt{R^{\prime} R^{\prime \prime}}
$$

and the effective Young's modulus is:

$$
E^{*}=\left(\frac{1-\vartheta_{1}^{2}}{E_{1}}+\frac{1-\vartheta_{2}^{2}}{E_{2}}\right)^{-1}
$$

where $\vartheta$ is the Poisson's ratio and $E$ is the Young's modulus for each cylinder.

When the adhesion effect is neglected, the Hertzian pressure distribution, $P$, acting in the elliptical contact area as obtained by [21], can be expressed as follows:

$$
P\left(r, \theta_{p}\right)=P o_{a b}\left[1-\left(r \cos \theta_{p} / a\right)^{2}-\left(r \sin \theta_{p} / b\right)^{2}\right]^{1 / 2}
$$

For this pressure distribution, the maximum pressure in the contact ellipse, $P o_{a b}$, is given as:

$$
P o_{a b}=\frac{E^{*}}{2 R} \frac{k^{2} a}{\beta\left\{\left[E(k) / \beta^{2}-K(k)\right][K(k)-E(k)]\right\}^{1 / 2}}
$$

where $\boldsymbol{K}(k)$ and $\boldsymbol{E}(k)$ are the complete elliptic integral of the first and second kind, and $k$ is the elliptic modulus (eccentricity of the ellipse). The value of $\beta$ throughout the contact is equal to $\beta_{0}$, the initial ellipticity ratio at initial loading that can be expressed as:

$$
\beta=\beta_{0}
$$

The total load compressing the cylinders, $W$, is related to the contact area as follows:

$$
W=\frac{4 E^{*}}{3 R F_{1}}(a b)^{3 / 2}
$$

where

$$
F_{1}=\left(\frac{4}{\pi k^{2}} \beta^{3 / 2}\left\{\left[\boldsymbol{E}(k) / \beta^{2}-\boldsymbol{K}(k)\right][\boldsymbol{K}(k)-\boldsymbol{E}(k)]\right\}^{1 / 2}\right)^{1 / 3}
$$

Due to the pressure in Eq. (7), the surface displacement within the ellipse of two bodies, $\omega$, is expressed in [21] as:

$$
\begin{gathered}
\omega_{i n_{a b}}\left(r, \theta_{p}\right)=\frac{1}{\pi E^{*}}\left\{\frac{\pi P o_{a b} a b}{2} \int_{0}^{\infty}\left[1-\frac{\left(r \cos \theta_{p}\right)^{2}}{a^{2}+w}-\frac{\left(r \sin \theta_{p}\right)^{2}}{b^{2}+w}\right] \frac{d w}{\left[\left(a^{2}+w\right)\left(b^{2}+w\right) w\right]^{1 / 2}}\right\} 0 \leq r \leq r_{a b} \\
\omega_{\text {out }_{a b}}\left(r, \theta_{p}\right)=\frac{1}{\pi E^{*}}\left\{\frac{\pi P o_{a b} a b}{2} \int_{l}^{\infty}\left[1-\frac{(12 \mathrm{a})}{a^{2}+w}-\frac{\left(r \sin \theta_{p}\right)^{2}}{b^{2}+w}\right] \frac{d w}{\left[\left(a^{2}+w\right)\left(b^{2}+w\right) w\right]^{1 / 2}}\right\} r \geq r_{a b}
\end{gathered}
$$

where $l$ is the positive root to $\frac{\left(r \cos \theta_{p}\right)^{2}}{a^{2}+l}+\frac{\left(r \sin \theta_{p}\right)^{2}}{b^{2}+l}=1$. 
Solutions for Eq. (12a) were obtained from [21]. Eq. (12b) was solved in [22] and is applied here in our model. Further solutions for Eq. (12b) can be found in Appendix B. Surface displacements in Eq. (12) are then rewritten as:

$$
\begin{aligned}
& \omega_{\text {in }_{a b}}\left(r, \theta_{p}\right)=\frac{1}{\pi E^{*}}\left[L_{\text {in }_{a b}}-M_{\text {in }_{a b}}\left(r \cos \theta_{p}\right)^{2}-N_{\text {in }_{a b}}\left(r \sin \theta_{p}\right)^{2}\right] \quad 0 \leq r \leq r_{a b} \\
& \omega_{\text {out }_{a b}}\left(r, \theta_{p}\right)=\frac{1}{\pi E^{*}}\left[L_{\text {out }_{a b}}-M_{\text {out }_{a b}}\left(r \cos \theta_{p}\right)^{2}-N_{\text {out }_{a b}}\left(r \sin \theta_{p}\right)^{2}\right] \quad r \geq r_{a b}
\end{aligned}
$$

where

$$
\begin{gathered}
L_{i n_{a b}}=\delta=\pi P_{o_{a b}} b \boldsymbol{K}(k) \\
M_{i n_{a b}}=\pi P_{o_{a b}} \beta[\boldsymbol{K}(k)-E(k)] / k^{2} a \\
N_{i n_{a b}}=\pi P_{o_{a b}} \beta\left[\boldsymbol{E}(k) / \beta^{2}-\boldsymbol{K}(k)\right] / k^{2} a
\end{gathered}
$$

and

$$
\begin{gathered}
L_{\text {out }_{a b}}=\pi P_{o_{a b}} b \boldsymbol{F}(\varphi, k) \\
M_{\text {out }_{a b}}=\pi P_{o_{a b}} \beta[\boldsymbol{F}(\varphi, k)-\boldsymbol{E}(\varphi, k)] / k^{2} a \\
N_{\text {out }_{a b}}=\pi P_{o_{a b}} \beta\left[\boldsymbol{E}(\varphi, k)-k^{\prime 2} \boldsymbol{F}(\varphi, k)-k^{2} \sin \varphi \cos \varphi / \sqrt{1-(k \sin \varphi)^{2}}\right] / k^{2} k^{\prime 2} a
\end{gathered}
$$

$\boldsymbol{F}(\varphi, k)$ and $\boldsymbol{E}(\varphi, k)$ are the incomplete elliptic integral of the first and second kind. $k^{\prime}$ is the complementary elliptic modulus and $\varphi$ is the second argument of the incomplete elliptic integrals.

\subsection{Extension of the double-Hertz theory to adhesive elliptical contacts}

We developed the adhesive elliptical contact model by extending the $\mathrm{DH}$ theory by [11], originally created for an adhesive circular contact. The basis of the $\mathrm{DH}$ model is that the adhesive tensile stresses are represented by the difference between two Hertzian pressure distributions of different radii (radial coordinates). The equations describing the adhesive stresses are given by:

$P\left(r, \theta_{p}\right)=P o_{c d}\left[1-\left(\frac{r \cos \theta_{p}}{c}\right)^{2}-\left(\frac{r \sin \theta_{p}}{d}\right)^{2}\right]^{1 / 2}-P o_{a b}\left[1-\left(\frac{r \cos \theta_{p}}{a}\right)^{2}-\left(\frac{r \sin \theta_{p}}{b}\right)^{2}\right]^{1 / 2} 0 \leq r \leq r_{a b}$

$$
P\left(r, \theta_{p}\right)=P o_{c d}\left[1-\left(\frac{r \cos \theta_{p}}{c}\right)^{2}-\left(\frac{r \sin \theta_{p}}{d}\right)^{2}\right]^{1 / 2} \quad r_{a b} \leq r \leq r_{c d}
$$

where $r_{a b}$ and $r_{c d}$ are the radial coordinates for the contact and the adhesive ellipses respectively, such that $r_{a b}<r_{c d}$. The contact ellipse is the Hertzian contact area that is due to the applied load. The additional pressure distribution creates the adhesive ellipse with semi-major axis $c$ and semi-minor axis $d$. Contact and adhesive ellipses bounded the 
adhesive region, an annular elliptical shaped area where the adhesion forces act. In this paper, it is assumed that both contact and adhesive ellipses have equal values of ellipticity ratio during contact, which is expressed as:

$$
\beta=\beta_{a b}=\beta_{c d}
$$

which at the pull-off moment, the relation in Eq. (17) becomes:

$$
\beta_{(p u l l-o f f)}=\beta_{a b_{(p u l l-o f f)}}=\beta_{c d(p u l l-o f f)}
$$

Following the Hertzian assumption for elliptical contacts, the relation of

$$
\beta=\beta_{(\text {pull-off })}=\beta_{0}
$$

is maintained throughout the adhesive contact.

The maximum pressure in the adhesive ellipse, $P O_{c d}$, is given as:

$$
P o_{c d}=\frac{E^{*}}{2 R} \frac{k^{2} c}{\beta\left\{\left[\boldsymbol{E}(k) / \beta^{2}-\boldsymbol{K}(k)\right][\boldsymbol{K}(k)-\boldsymbol{E}(k)]\right\}^{1 / 2}}
$$

which is similar in form as Eq. (8) of the contact ellipse.

The surface displacements are:

$$
\begin{array}{cl}
\omega_{0 a b}\left(r, \theta_{p}\right)=\omega_{i n_{a b}}-\omega_{i n_{c d}} & 0 \leq r \leq r_{a b} \\
\omega_{a b c d}\left(r, \theta_{p}\right)=\omega_{o u t_{a b}}-\omega_{i n_{c d}} & r_{a b} \leq r \leq r_{c d}
\end{array}
$$

Fig. 3(a) shows the normalized pressure $P / P_{0}$ curves, obtained using Eqs. (8), (16) and (20) at $\beta_{0}=0.99$, which corresponds to $\theta_{\text {skew }} \approx 90^{\circ}$. From Fig. 3(a), the maximum adhesive stress can be seen to occur at $r_{a b}$ and then decreases to approach a zero value at $r_{c d}$. Surface displacements at $\beta_{0}=0.99$ are also presented in Fig. 3(b), using Eq. (21). The results of the original $\mathrm{DH}$ model for a circular contact are also included. A uniform displacement over the contact region is annulled by a rigid-body displacement over the adhesive region of $r_{a b} \leq r \leq r_{c d}$ to leave a gap. It can be observed that the surface deformations for the current model at $\beta_{0}=0.99$ closely resemble those of the $\mathrm{DH}$ model for a circular contact. 


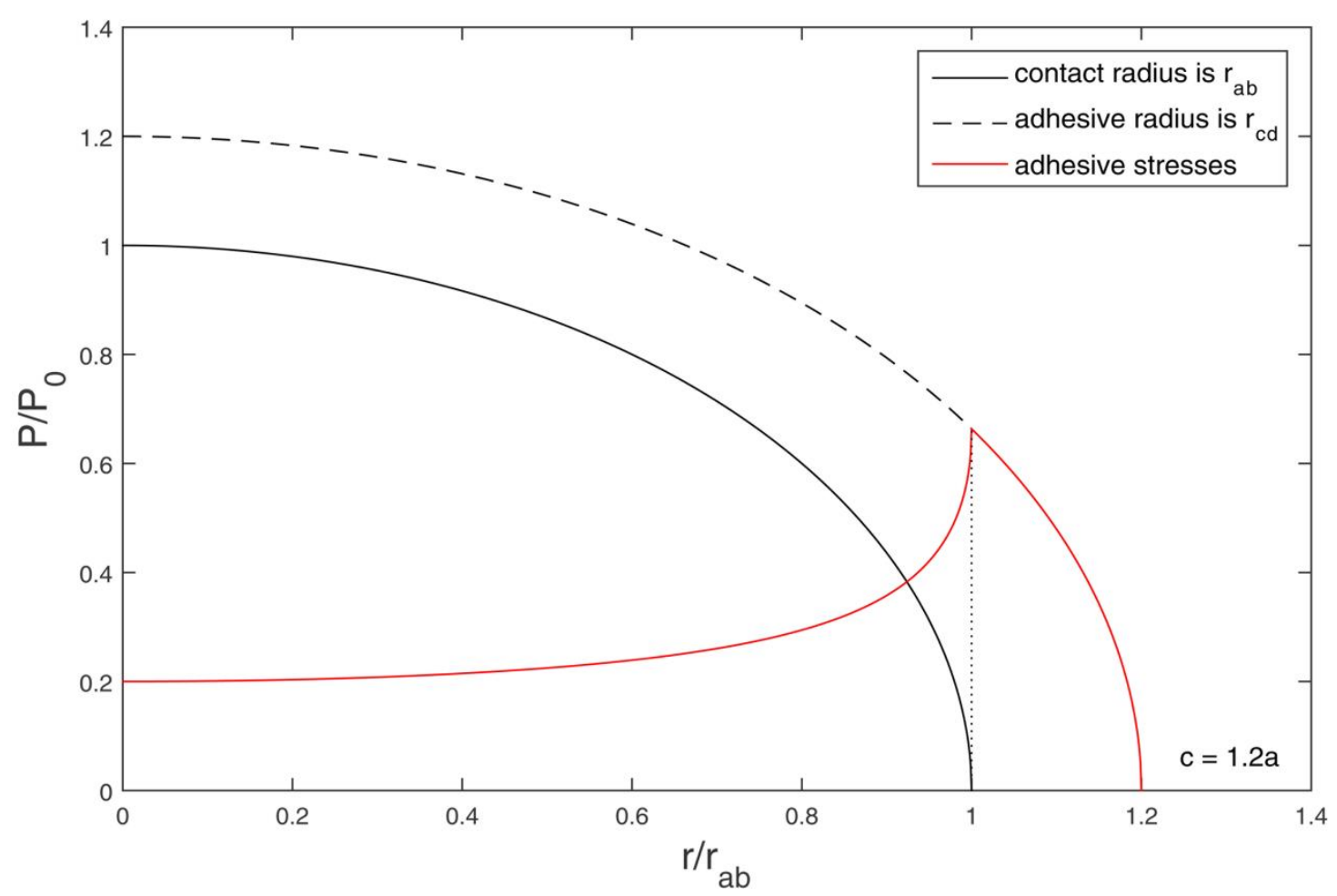

(a)

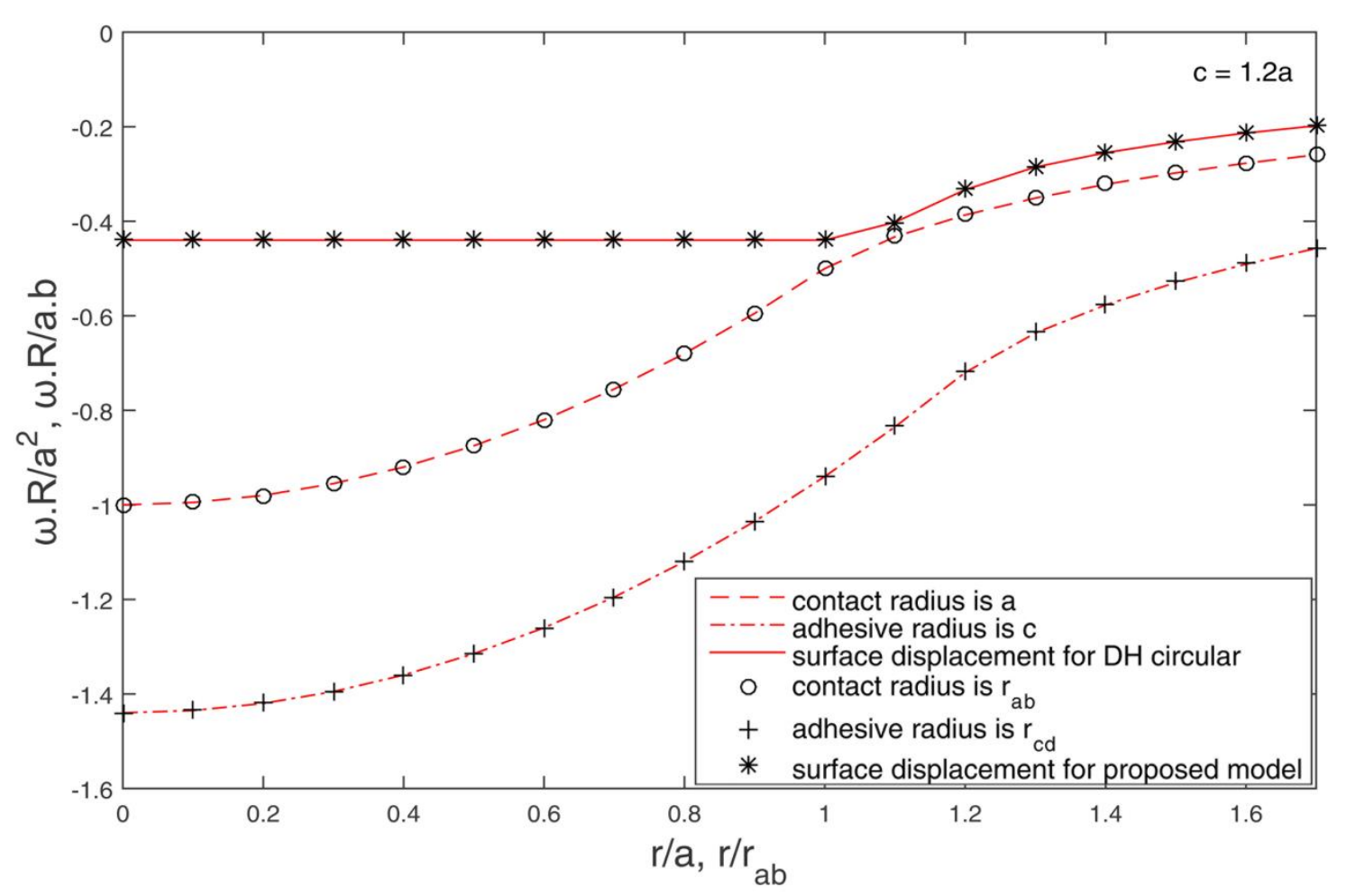

(b)

Fig. 3: (a) The pressure difference between two Hertzian solutions (b) Surface displacements for both the $\mathrm{DH}$ circular model and the developed model. 
In a similar way as in the original DH model, the pressure expressions in Eq. (16) are scaled by $v$ to model the adhesive tensile stresses over the adhesive region, $r_{a b} \leq r \leq r_{c d}$, which produces the final stresses distribution when combined with an unscaled Hertzian pressure. The scaling factor $v$ is determined by the surface forces, which will be further discussed in Section 2.3. The pressure equations are now expressed as:

$$
\begin{gathered}
P\left(r, \theta_{p}\right)= \\
v\left\{P o_{c d}\left[1-\left(\frac{r \cos \theta_{p}}{c}\right)^{2}-\left(\frac{r \sin \theta_{p}}{d}\right)^{2}\right]^{1 / 2}-P o_{a b}\left[1-\left(\frac{r \cos \theta_{p}}{a}\right)^{2}-\left(\frac{r \sin \theta_{p}}{b}\right)^{2}\right]^{1 / 2}\right\} \quad 0 \leq r \leq r_{a b}
\end{gathered}
$$

$$
P\left(r, \theta_{p}\right)=v P o_{c d}\left[1-\left(\frac{r \cos \theta_{p}}{c}\right)^{2}-\left(\frac{r \sin \theta_{p}}{d}\right)^{2}\right]^{1 / 2} \quad r_{a b} \leq r \leq r_{c d}
$$

From Eq. (22b), it can be seen that the distribution of the tensile stress over the adhesive annular region is given by:

$$
\sigma_{a c}\left(r, \theta_{p}\right)=-P\left(r, \theta_{p}\right)=-v P o_{c d}\left[1-\left(\frac{r \cos \theta_{p}}{c}\right)^{2}-\left(\frac{r \sin \theta_{p}}{d}\right)^{2}\right]^{1 / 2}
$$

Then, at $r=a$ and $\theta_{p}=0^{\circ}$, the maximum adhesive stress is given by:

$$
\sigma_{0}=-v P o_{c d}\left(1-\frac{a^{2}}{c^{2}}\right)^{1 / 2}
$$

The combined load, including the load in Eq. (10), is expressed as:

$$
W=\frac{4 E^{*}}{3 R F_{1}}\left\{(a b)^{3 / 2}-v\left[(c d)^{3 / 2}-(a b)^{3 / 2}\right]\right\}
$$

To include the scaling factor $v$, surface displacements in Eq. (21) are rewritten as:

$$
\begin{array}{cl}
\omega_{0 a b}\left(r, \theta_{p}\right)=v\left(\omega_{i n_{a b}}-\omega_{i n_{c d}}\right) & 0 \leq r \leq r_{a b} \\
\omega_{a b c d}\left(r, \theta_{p}\right)=v\left(\omega_{o u t_{a b}}-\omega_{i n_{c d}}\right) & r_{a b} \leq r \leq r_{c d}
\end{array}
$$

The gap, $h$, for the area outside the contact where $r_{a b} \leq r \leq r_{c d}$ is then given by:

$$
h\left(r, \theta_{p}\right)=z+\omega_{o u t_{a b}}+\omega_{a b c d}-\omega_{0 a b}-\delta
$$




\subsection{Work of adhesion}

The work needed to break the intermolecular bonds at the pull-off moment is termed work of adhesion. This work is required to create new surfaces when separating two bodies attached together due to the presence of the adhesion forces. The work of adhesion from [11] can be expressed as:

$$
\Delta \gamma=\int_{0}^{\infty} \sigma_{a c} d h
$$

which becomes

$$
\Delta \gamma=\int_{0}^{\infty} \int_{0}^{\sigma_{a c}(h)} d \sigma_{a c} d h
$$

where $\sigma_{a c}(h)$ is dependent on the separation $h$. For an elliptical contact, $h$ is a function of both $r$ and $\theta_{p}$, as shown in Eq. (27). Using the Jacobian of the transformation, the work of adhesion for an adhesive elliptical contact from Eq. (29) can be expressed as:

$$
\Delta \gamma=4 \int_{0}^{\pi / 2} \int_{r_{a b}}^{r_{c d}}\left(\frac{d \sigma_{a c}}{d r} \frac{d h}{d \theta_{p}}-\frac{d \sigma_{a c}}{d \theta_{p}} \frac{d h}{d r}\right) d r d \theta_{p}
$$

where the scaling factor $v$ is chosen such that the values of $\sigma_{0}$ and $\Delta y$ are fixed.

In this paper, only the results for the angular coordinate at $\theta_{p}=0^{\circ}$ are shown, resulting in the model having a one-dimensional solution for the contact problem. The expression for the work of adhesion in Eq. (30) for $\theta_{p}=0^{\circ}$ can be rewritten as:

$$
\Delta \gamma=\int_{r_{a b}}^{r_{c d}} \sigma_{a c}(r) \frac{d h}{d r} d r
$$

\section{Results}

Previous results are summarized in non-dimensional form following the work of [11] and [23] by:

$$
a=c_{r} a^{*} ; b=c_{r} b^{*} ; c=c_{r} c^{*} ; d=c_{r} d^{*}
$$

and

$$
\begin{gathered}
c_{r}{ }^{3}=R^{2} \Delta \gamma / E^{*} \\
W=2 \pi R \Delta \gamma W^{*} / \sin \theta_{\text {skew }}
\end{gathered}
$$

Further, the Tabor parameter is defined in [10] as:

$$
\mu=\sigma_{0}\left(R / E^{* 2} \Delta \gamma\right)^{1 / 3}
$$

Using the equations above, the normal load in Eq. (25) is now expressed in nondimensional form as:

$$
W^{*}=\frac{2}{3 \pi} \frac{\sin \theta_{\text {skew }}}{F_{1}^{3}}\left\{\left(a^{*} b^{*}\right)^{3 / 2}-v\left[\left(c^{*} d^{*}\right)^{3 / 2}-\left(a^{*} b^{*}\right)^{3 / 2}\right]\right\}
$$


The effects of changing the normalized load $W^{*}$ on the semi-major axes of contact and adhesive ellipses, $a^{*}$ and $c^{*}$, are shown in Fig. 4 and Fig. 5. We obtained the values of $c^{*}$ and the scaling factor $v$ by solving Eqs. (31) and (35) simultaneously in MATLAB. Due to incomplete elliptic integrals in Eq. (31), it cannot be solved directly in the same manner as in the $\mathrm{DH}$ circular contact. Both $c^{*}$ and $v$ values were then used in Eq. (36) to calculate the corresponding load. At a nearly circular contact, the model is compared to the original $\mathrm{DH}$ model for an intermediate case of $\mu=1$ and the JKR circular model for the soft material comparison at $\mu=5$. A $\beta_{0}$ value of 0.99 follows the behavior of a circular contact accurately for all $\mu$ values. At $\mu=0.5$, the pull-off moment occurs at a near-zero contact with a high pull-off force. It is shown that the deformations due to adhesion forces are negligible, which is in agreement with the predictions of the DMT model. For $\mu=5$, the surface separation involves a low pull-off force at an apparent non-zero contact, which is similar to those predicted by the JKR model. In Fig. 5, $c^{*}$ values are shown to be highly influenced by the value of $\mu$, such that they become smaller with increasing $\mu$. As the $\mu$ values approach the JKR domain where materials are easily deformed, the adhesive ellipse becomes smaller while the contact ellipse becomes larger, resulting in a narrow adhesive region. This is in close agreement with the adhesive behavior in the JKR domain, where adhesion is contained within the contact area. 


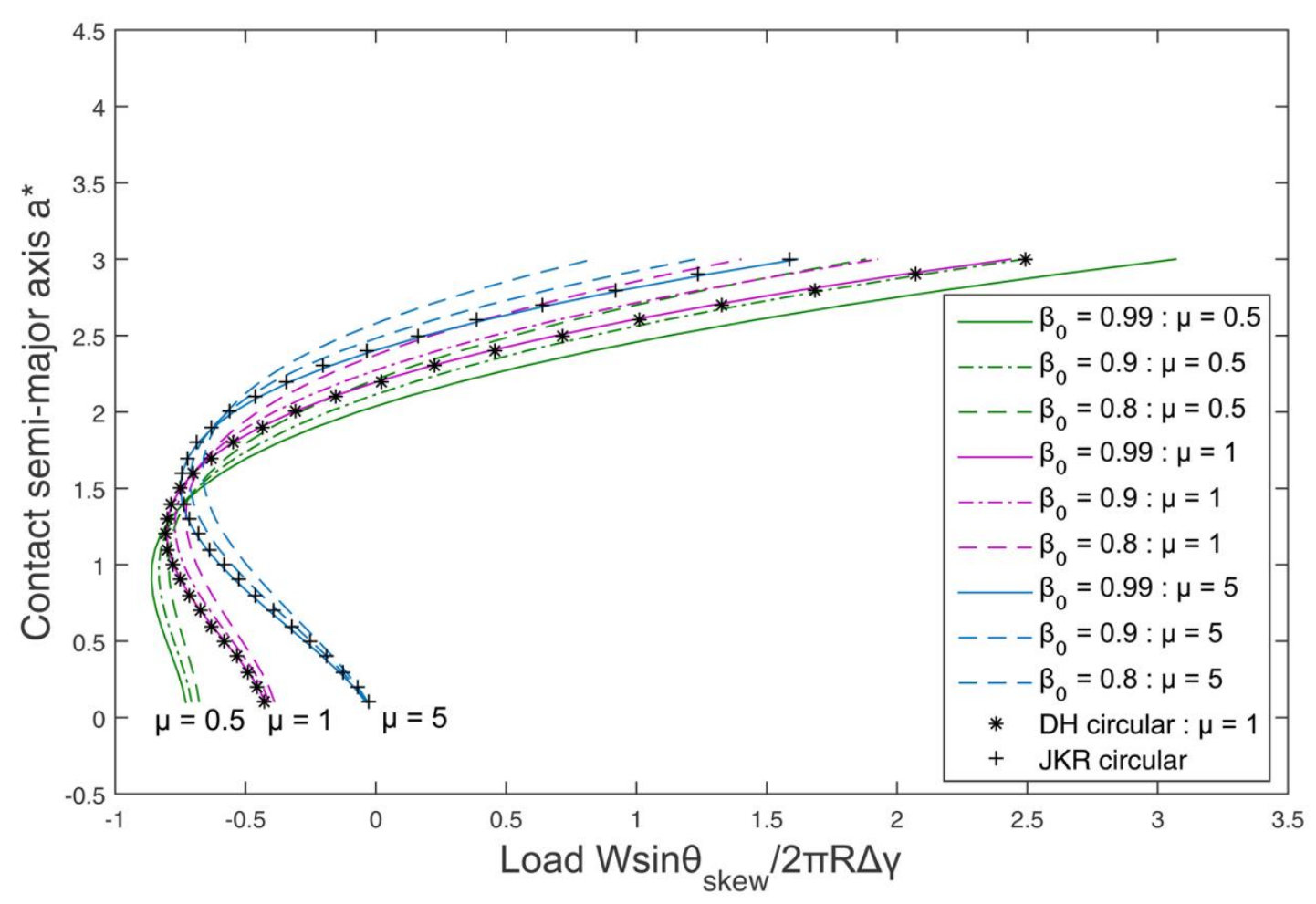

Fig. 4: Variation of the contact semi-major axis $a^{*}$ with the normalized load $W^{*}$ for various values of $\mu$.

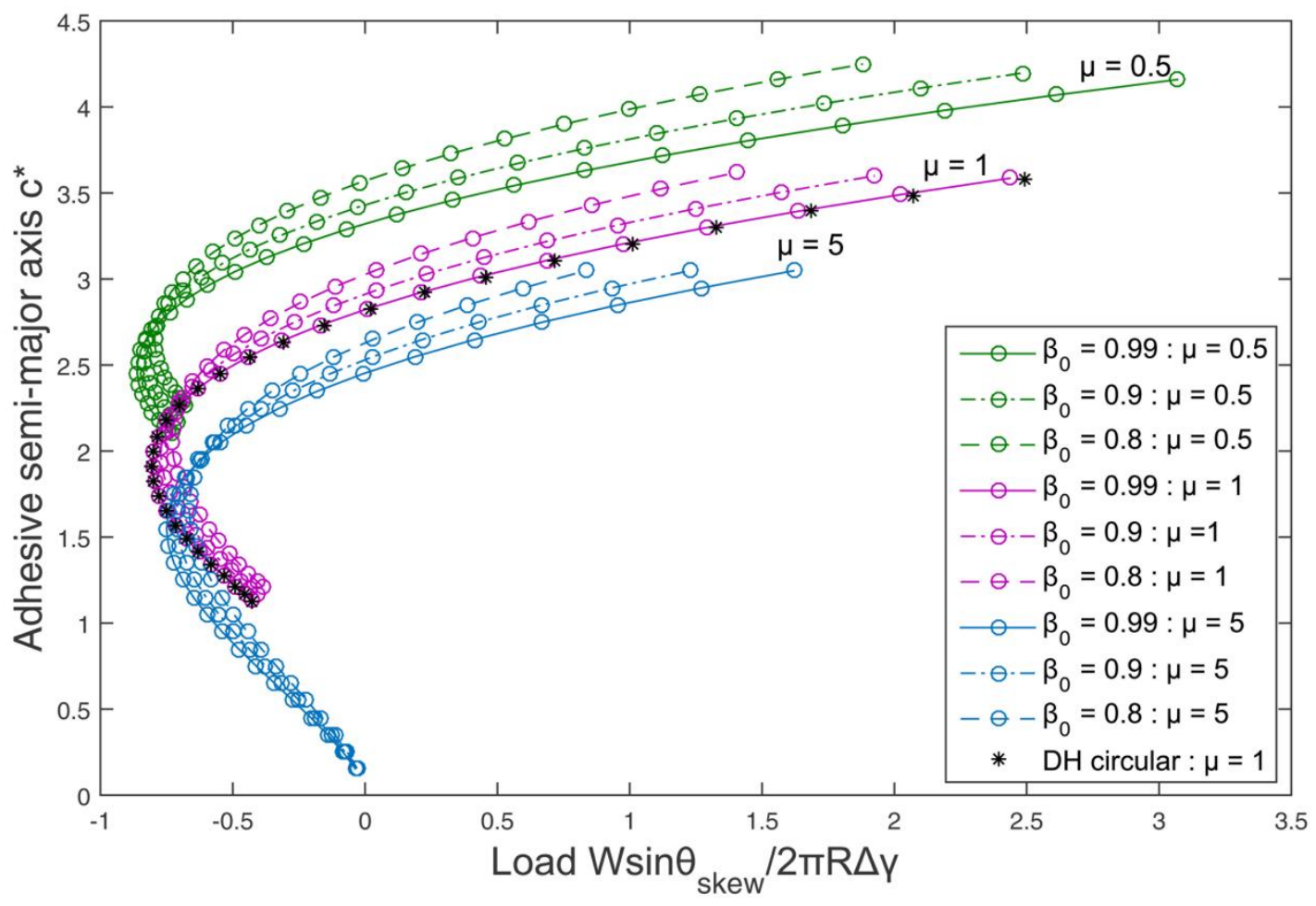

Fig. 5: Variation of the adhesive semi-major axis $c^{*}$ with the normalized load $W^{*}$ for various values of $\mu$. 
The performance of the developed model is further investigated for particular $\mu$ groups, as shown in Fig. 6 and Fig. 7. Both graphs plot the semi-major axes of contact and adhesive ellipses, $a^{*}$ and $c^{*}$, versus the normalized load $W^{*}$. Results are shown for various $\beta_{0}$ values at $\mu=0.5$ and $\mu=5$ respectively. At $\mu=0.5$, it is shown that with decreasing $\beta_{0}$ values, the gap between $a^{*}$ and $c^{*}$ becomes considerably wider. This shows that, outside the contact area, an expansion of the adhesive region along the major axis is predicted for contacts which deviate from a circular shape. However, there is barely any effect at $\mu=5$, as shown in Fig. 7. At $\mu=5$, a values are nearly equal to $c^{*}$ values, indicating a narrow adhesive region.

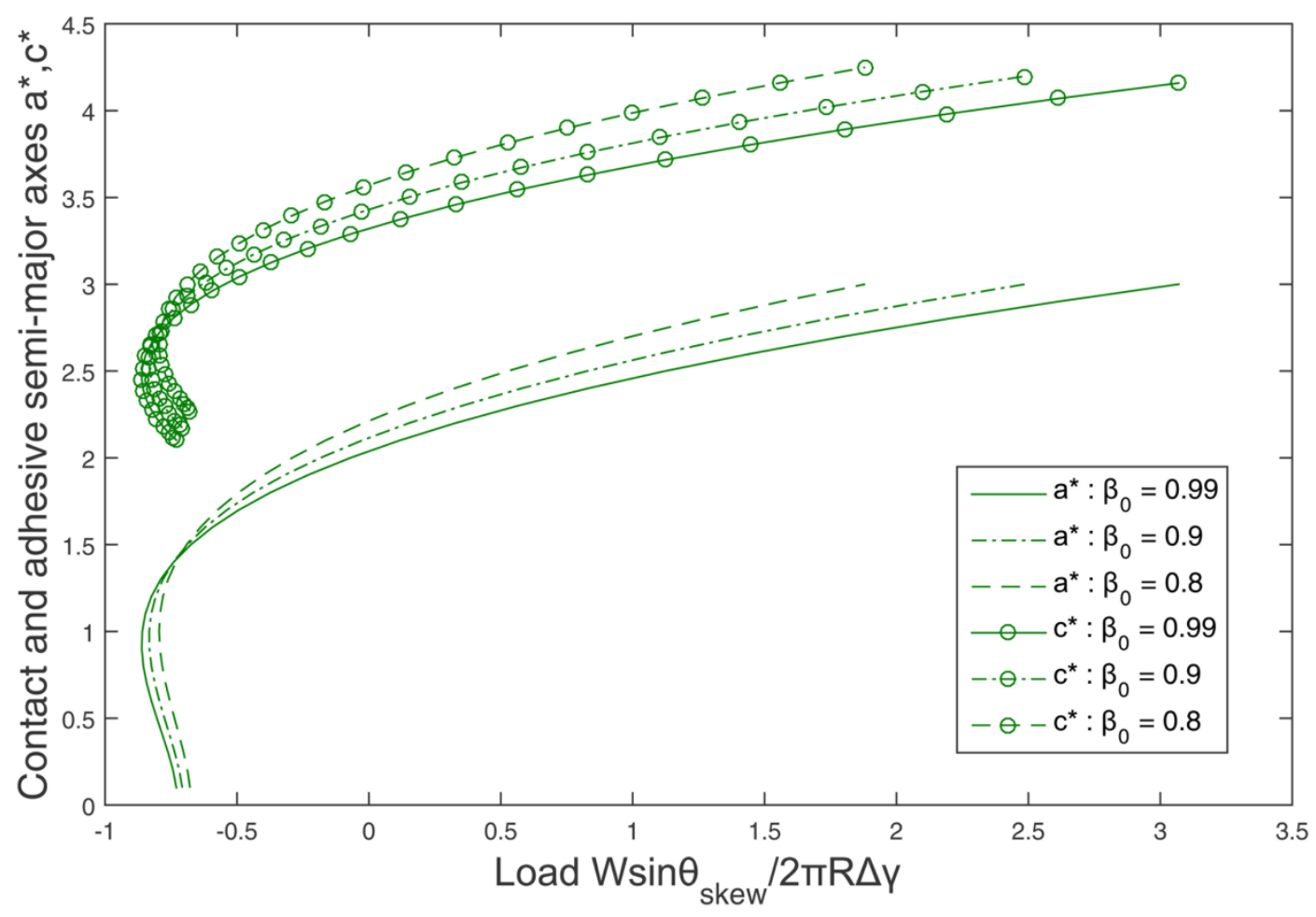

Fig. 6: Contact and adhesive semi-major axes $a^{*}$ and $c^{*}$ versus the normalized load $W^{*}$ for various $\beta_{0}$ values at $\mu=0.5$. 


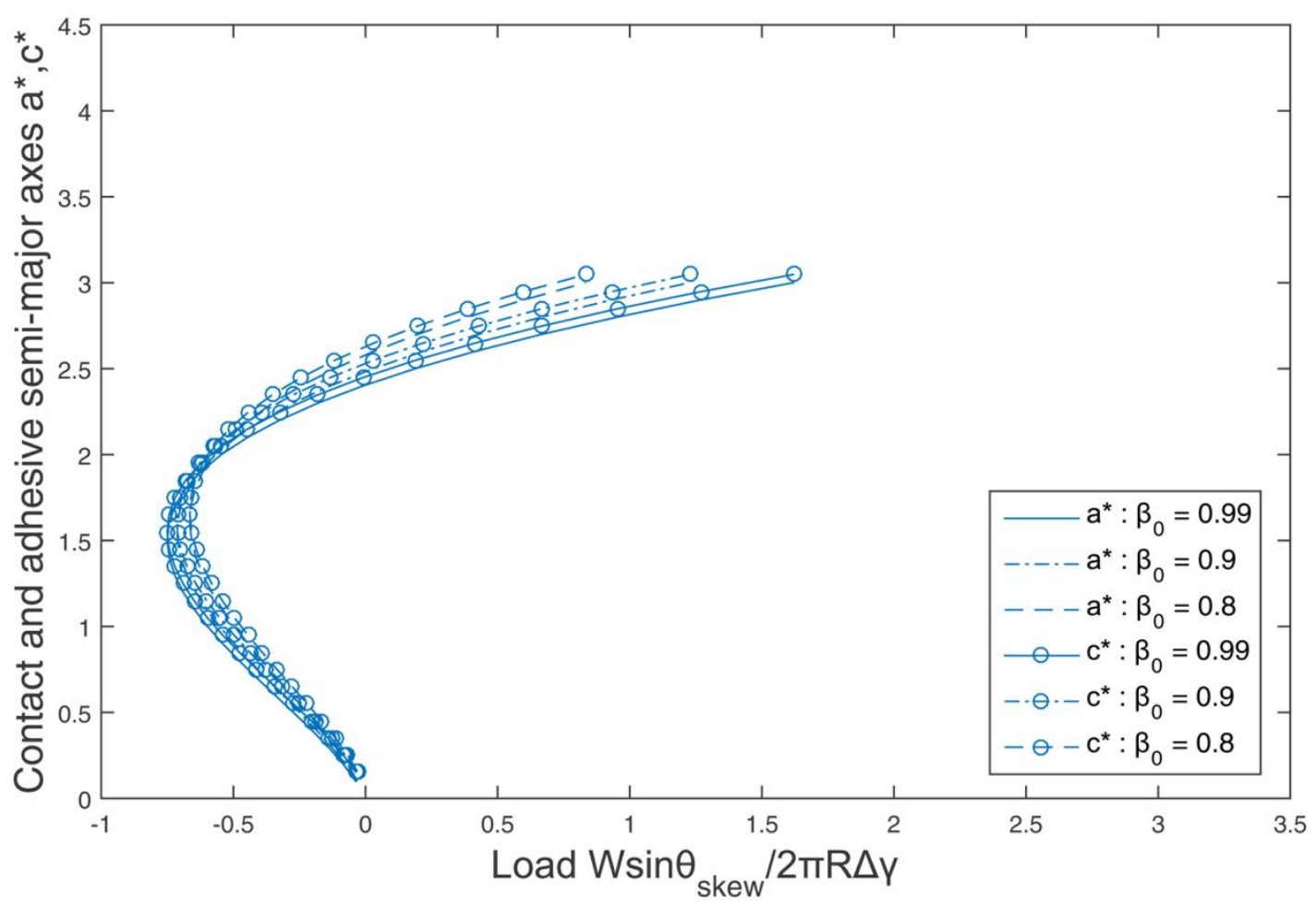

Fig. 7: Contact and adhesive semi-major axes $a^{*}$ and $c^{*}$ versus the normalized load $W^{*}$ for various $\beta_{0}$ values at $\mu=5$.

In Fig. 8, the model is compared to the JKR elliptical model at its adhesive domain of $\mu=5$. Only the curve of $\beta_{0}=0.99$ has a close fit to the JKR elliptical model. The difference in pull-off force values between both models is more apparent with decreasing $\beta_{0}$. Although both models show similar deformations, the pull-off forces predicted by the developed model at $\beta_{0}$ values of 0.8 and 0.9 are considerably low for $\beta_{0}$ values that are considered to be close to one. 


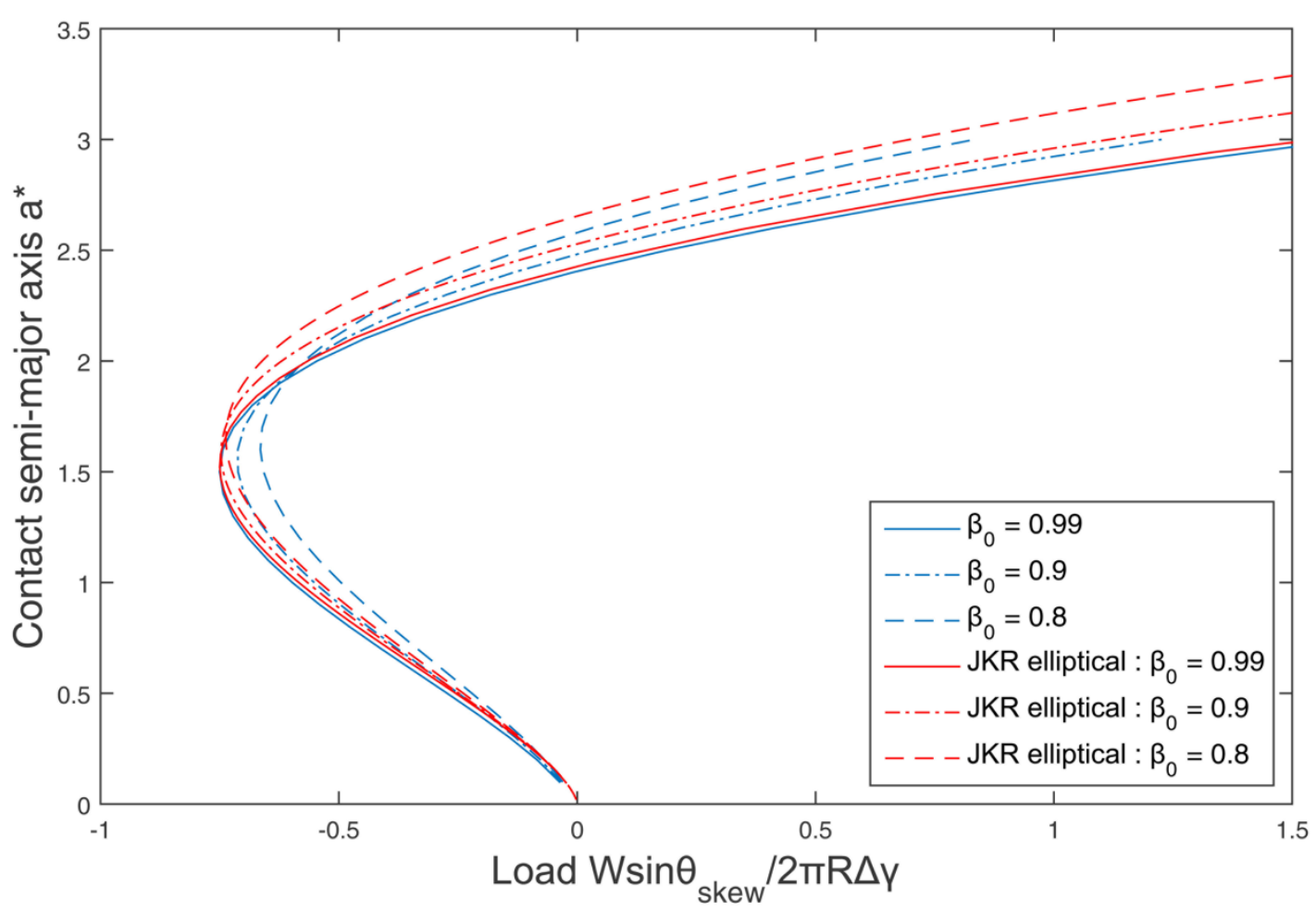

Fig. 8: Variation of the contact semi-major axis $a^{*}$ with the normalized load $W^{*}$ for various $\beta_{0}$ values at $\mu=5$.

As previously shown in Fig. 8, the initial assumption of an adhesive region with both contact and adhesive ellipses having equal and constant ellipticity ratios as the load varies, as given in Eqs. (17) and (19), is not realistic for $\beta_{0}$ values of 0.8 and 0.9. To further analyse the behavior of the adhesive region during contact, the pull-off moment results from the assumption of Eq. (19) are compared to when the model employed JKR-like behavior, in which its ellipticity ratio is constantly changing as the load varies. At the pulloff moment, both results employ Eq. (18) for the relation between the contact and adhesive ellipses. For the JKR-like behavior, the ellipticity ratios of both contact and adhesive ellipses are made equal as the ellipticity ratio of the contact area obtained from

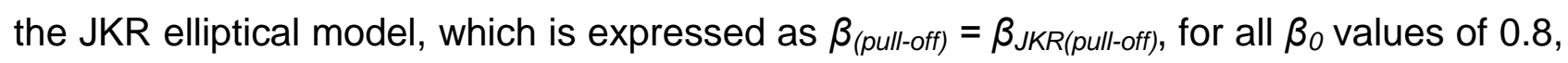
0.9 and 0.99 .

The variation of the normalized pull-off force $W_{\max }{ }^{*}$ with the Tabor parameter $\mu$ is shown in Fig. 9(a). Results from both assumptions employed in the developed model are compared with the existing adhesive circular and elliptical models. Both results from the developed model produce curves which lie close to the other adhesive circular and elliptical models at $\beta_{0}=0.99$. At $\beta_{0}$ values of 0.8 and 0.9 , the assumption of Eq. (19) produces results which clearly deviate from the JKR elliptical model. With the new 
assumption of JKR-like behavior, the gap between the predicted pull-off forces of the developed model and the JKR values is slightly improved for $\beta_{0}$ values of 0.8 and 0.9 , as shown in the relative errors graph in Fig. 9(b). It must be noted that a perfect fit between the JKR curves and the developed model cannot be expected in the JKR domain. The adhesive force for the JKR model is restricted within its contact area, which is different from the adhesive behavior outside the JKR domain, where the adhesive region is present outside the contact ellipse. This is an important characteristic of the developed model that must be taken into consideration. 


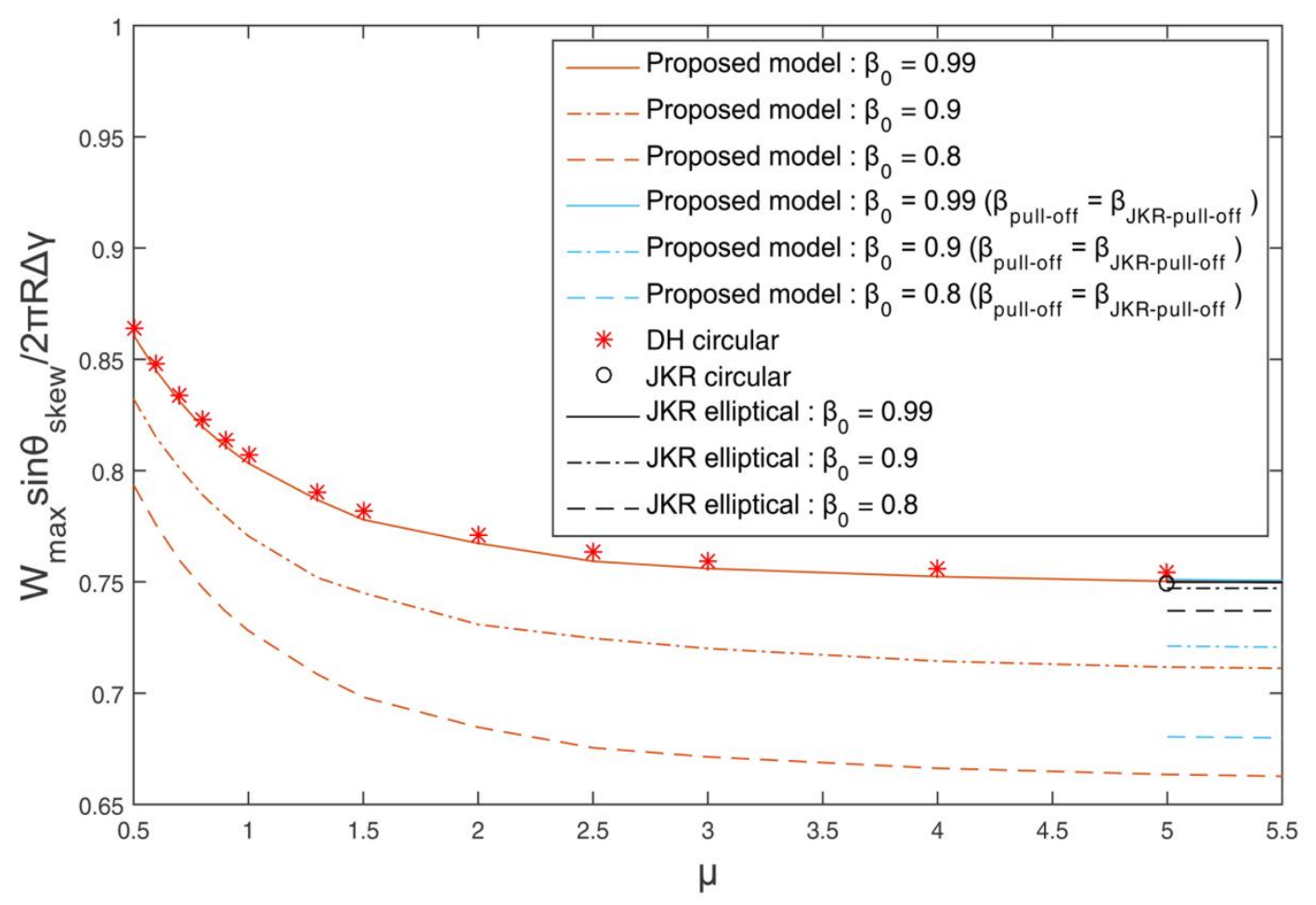

(a)

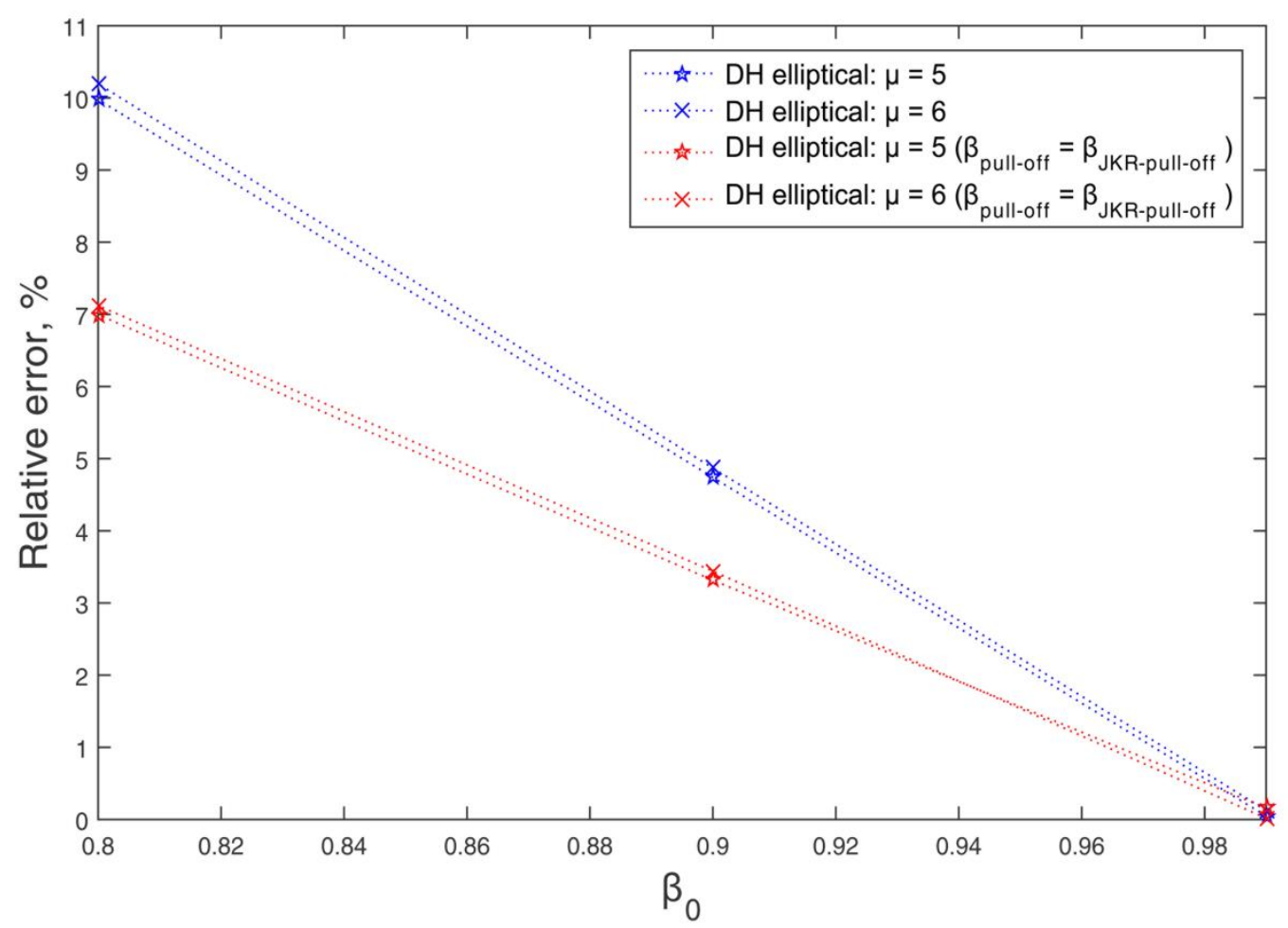

(b)

Fig. 9: (a) Normalized pull-off force $W_{\max }{ }^{*}$ as a function of the Tabor parameter $\mu$ for various ellipticity ratios $\beta_{0}$ (b) The percentage of relative error for both results from the developed model when compared to the JKR elliptical model in the JKR domain. 


\section{Discussion}

At $\beta_{0}$ values of 0.8 and 0.9 , results from the developed model show an apparent deviation for the nearly circular contact comparison. Using the initial assumption of $\beta=\beta_{\text {(pull-off) }}=\beta_{0}$ throughout the contact until the pull-off moment, the adhesive region becomes smaller as the load decreases but it maintains its annular elliptical shape. This is due to a constant deformation rate along the major and minor axes directions for both the contact and adhesive ellipses. As for the JKR contact, which assumes a constantly changing $\beta$ during contact $\left(\beta_{(\text {pull-off })} \neq \beta_{0}\right)$, the shape of the contact area that contains the adhesive stresses slowly changes from an ellipse to a nearly circular shape at the pull-off moment. From the initial load, the deformation rate along the minor axis of the elliptical contact slowly increases, the shape of which eventually turns into a nearly circular contact area during pull-off. Adhesion that acts within a nearly circular contact area requires a higher pull-off force for the separation compared to a narrow annular elliptical-shaped adhesive region. Fig. 10 illustrates the evolution of the adhesive region and the contact area for the developed model with $\beta=\beta_{\text {(pull-off) }}=\beta_{0}$ assumption, including the JKR contact area as a comparison. The assumption of identical, fixed ellipticity ratios throughout the contact for the contact and adhesive ellipses which bounded the adhesive region is shown to be unsuitable for the pull-off force prediction, as seen in the limiting JKR case where the pulloff force is underestimated.

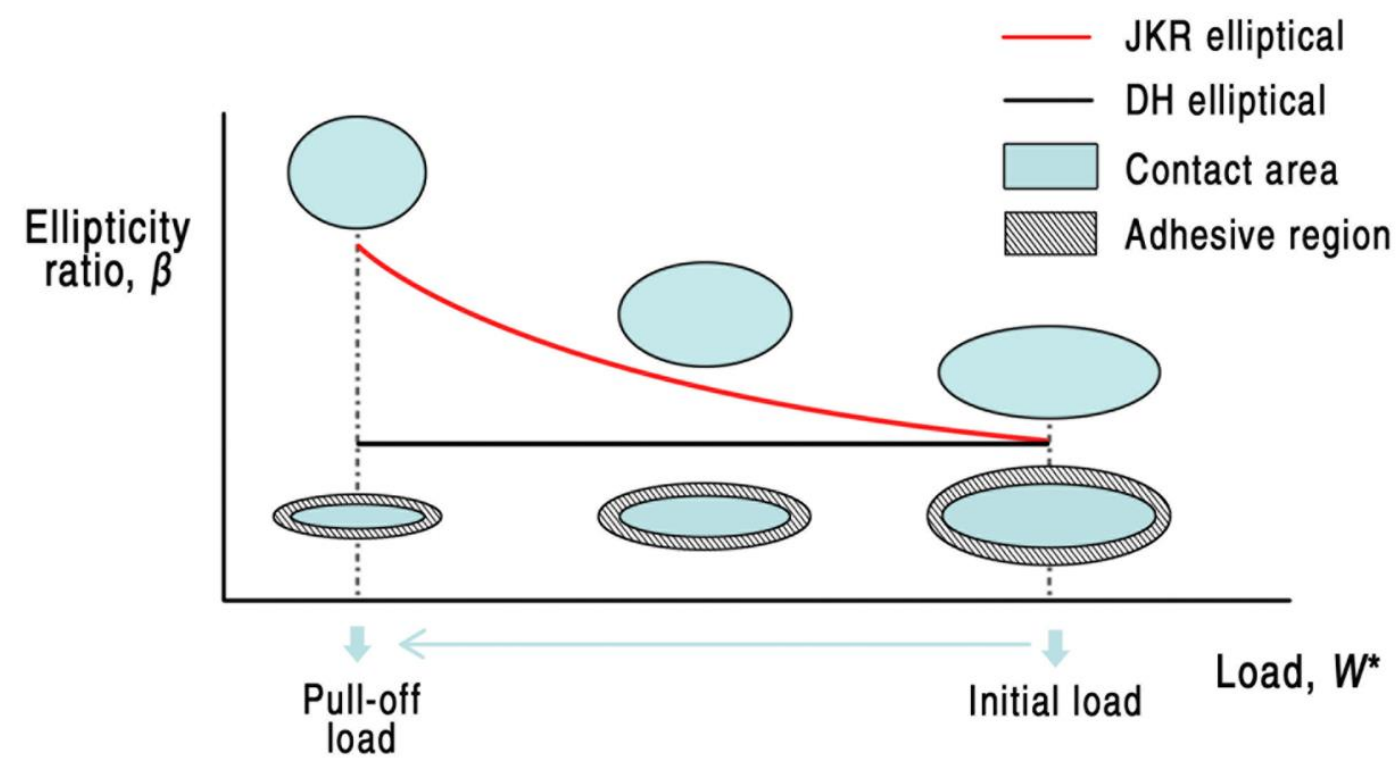

Fig. 10: Evolution of the adhesive region and the contact area from initial loading to pull-off moment for both the JKR and the proposed model. 
At the JKR domain, pull-off moment results from the new assumptions $\left(\beta_{(\text {pull-off })}=\right.$ $\left.\beta_{J K R(p u l l-o f f)}\right)$ are closer to the JKR elliptical model, compared to those from the initial assumption of $\beta=\beta_{\text {(pull-off) }}=\beta_{0}$. The results provide insight into how the developed model should behave near the JKR domain, which has ellipticity ratios that are continuously changing as the load varies. At $\mu=5$, a smooth transition cannot be expected between the developed model with either the initial or the new assumption and the JKR elliptical model, because the developed model cannot model the adhesion inside the contact area, which is the foundation of the JKR model.

Additionally, assuming an equal value of $\beta$ for both contact and adhesive ellipses $\left(\beta=\beta_{a b}=\beta_{c d}\right)$ over a wide range of $\mu$ is also unrealistic. It is important to find the real geometrical behavior of both contact and adhesive ellipses to improve the model's prediction, regardless of material types and crossing angles. It is expected that the adhesive ellipse has the same ellipticity ratio as the contact ellipse for low $\mu$ values, while nearly circular adhesive regions are expected for high $\mu$ values that are closer to the JKR domain. Further work is essential to predict realistic geometrical behavior of contact and adhesive ellipses to enable accurate prediction for an adhesive elliptical contact. Numerical simulations such as [20,24] have been shown to be able to model adhesive contact accurately, which can help us to predict the behavior of both contact and adhesive ellipses.

\section{Conclusions}

A model has been developed for predicting the adhesive elliptical contact by extending the DH model. Both contact and adhesive ellipses have identical, fixed ellipticity ratios, following the Hertzian assumption. Based on this, the geometry of the adhesive contact can be modeled, allowing pull-off force predictions for adhesive elliptical contacts. The results are in agreement with those obtained using existing adhesive circular and elliptical models, but only at nearly circular contacts. Mildly elliptical contacts have better results when the adhesive region is assumed to be constantly changing, as taken into account in the JKR model. This behavior is also expected for non-JKR contacts, based on the transition from the JKR model to the developed model in the pull-off force prediction.

\section{Acknowledgments}

The authors gratefully acknowledge support from the Ministry of Education Malaysia, Universiti Teknikal Malaysia Melaka and Green Tribology and Engine Performance (GTriboE) research group. 
Appendix A: Coordinate system for governing equations

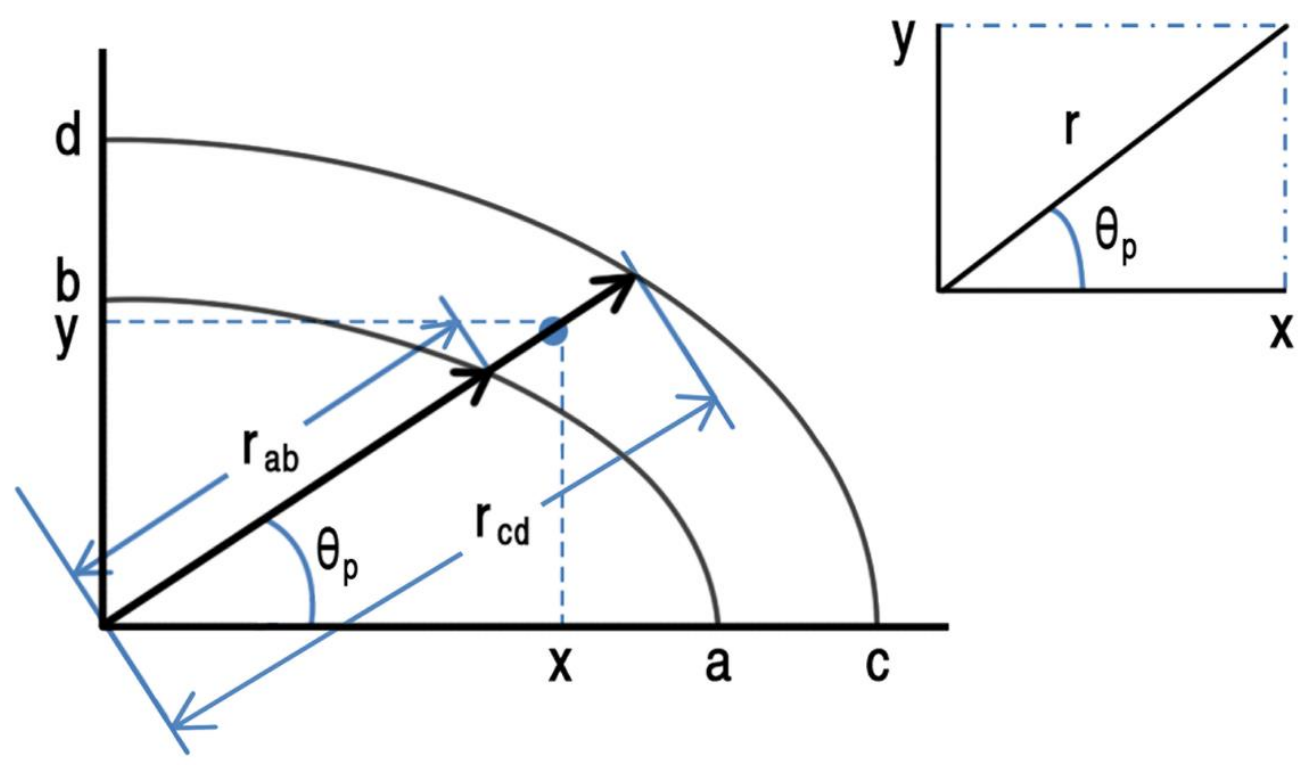

Fig. 11: Cartesian to polar coordinate system transformation for the contact and adhesive ellipses.

$$
\begin{aligned}
& r_{a b}=a b / \sqrt{\left(b \cos \theta_{p}\right)^{2}+\left(a \sin \theta_{p}\right)^{2}} \\
& r_{c d}=c d / \sqrt{\left(d \cos \theta_{p}\right)^{2}+\left(c \sin \theta_{p}\right)^{2}}
\end{aligned}
$$

where

$$
\begin{aligned}
& x=r \cos \theta_{p} \\
& y=r \sin \theta_{p}
\end{aligned}
$$

Appendix B: Solutions for incomplete elliptic integrals

$$
\begin{gathered}
I_{1}=\int_{l}^{\infty} \frac{\mathrm{dw}}{\left[\left(a^{2}+w\right)\left(b^{2}+w\right) w\right]^{1 / 2}}=\frac{2 \boldsymbol{F}(\varphi, k)}{a} \\
I_{2}=\int_{l}^{\infty} \frac{\mathrm{dw}}{\left[\left(a^{2}+w\right)^{3}\left(b^{2}+w\right) w\right]^{1 / 2}}=\frac{2[\boldsymbol{F}(\varphi, k)-E(\varphi, k)]}{k^{2} a^{3}} \\
I_{3}=\int_{l}^{\infty} \frac{\mathrm{dw}}{\left[\left(a^{2}+w\right)\left(b^{2}+w\right)^{3} w\right]^{1 / 2}}=\frac{2}{k^{2} k^{\prime 2} a^{3}}\left[\boldsymbol{E}(\varphi, k)-k^{\prime 2} \boldsymbol{F}(\varphi, k)-\frac{k^{2} \sin \varphi \cos \varphi}{\sqrt{1-(k \sin \varphi)^{2}}}\right] \\
L_{o u t_{a b}}=\frac{\pi P_{o_{a b}} a b}{2} I_{1} \\
M_{\text {out }_{a b}}==\frac{\pi P_{a b} a b}{2} I_{2} \\
N_{\text {out }_{a b}}=\frac{\pi P_{O_{a b}} a b}{2} I_{3}
\end{gathered}
$$




\section{References}

1. Shi, Q., Wong, S., Ye, W., Hou, J., Zhao, J., Yin, J.: Mechanism of adhesion between polymer fibers at nanoscale contacts. Langmuir 28(10), 4663-4671 (2012)

2. Lai, T., Huang, P.: Study on microscale adhesion between solid surfaces with scanning probe. Sci. China Technol. Sci. 56, 2934-2952 (2013)

3. Lai, T., Chen, R., Huang, P.: Temperature dependence of microscale adhesion force between solid surfaces using an AFM. J. Adhes. Sci. Technol. 29(2), 133-148 (2014)

4. Hanrahan, B., Misra, S., Beyaz, M., Feldman, J., Waits, C., Ghodssi, R.: An adhesion-dominated rolling friction regime unique to micro-scale ball bearings. Tribol. Lett. 56(2), 215-221 (2014)

5. Kendall, K.: Adhesion: Molecules and mechanics. Science 263(5154), 1720-1725 (1994)

6. Johnson, K.L., Kendall, K., Roberts, A.D.: Surface energy and the contact of elastic solids. Proc. R. Soc. Lond. Ser. A 324, 301-313 (1971)

7. Derjaguin, B.V., Muller, V.M., Toporov, Y.P.: Effect of contact deformations on the adhesion of particles. J. Colloid Interface Sci. 53,314-325 (1975)

8. Muller, V.M., Derjaguin, B.V., Toporov, Y.P.: On two methods of calculation of the force of sticking of an elastic sphere to a rigid plane. Colloids Surf. 7, 251-259 (1983)

9. Maugis, D.: Adhesion of spheres: The JKR-DMT transition using a Dugdale model. J. Colloid Interface Sci. 150(1), 243-269 (1992)

10. Tabor, D.: Surface forces and surface interactions. J. Colloid Interface Sci. 58(1), 213 (1977)

11. Greenwood, J., Johnson, K.: An alternative to the Maugis model of adhesion between elastic spheres. J. Phys. D Appl. Phys. 31(22), 3279-3290 (1998)

12. Barquins, M.: Adherence and rolling kinetics of a rigid cylinder in contact with a natural rubber surface. J. Adhes. 26(June), 1-12 (1988)

13. Baney, J., Hui, C.: A cohesive zone model for the adhesion of cylinders. J. Adhes. Sci. Technol. 11(3), 393-406 (1997)

14.Johnson, K., Greenwood, J.: Maugis analysis of adhesive line contact. J. Phys. D Appl. Phys. 41(19), 199802-199802 (2008)

15. Jin, F., Zhang, W., Zhang, S., Guo, X.: Adhesion between elastic cylinders based on the double-Hertz model. Int. J. Solids Struct. 51(14), 2706-2712 (2014) 
16.Zhang, W., Jin, F., Zhang, S., Guo, X.: Adhesive contact on randomly rough surfaces based on the Double-Hertz model. J. Appl. Mech. 81(5),051008 (2014)

17.Jin, F., Wan, Q., Guo, X.: A double-Westergaard model for adhesive contact of a wavy surface. Int. J. Solids Struct.102-103, 66-76 (2016)

18. Johnson, K., Greenwood, J.: An approximate JKR theory for elliptical contacts. J. Phys. D Appl. Phys. 38(7), 1042-1046 (2005)

19.Sümer, B., Onal, C., Aksak, B., Sitti, M.: An experimental analysis of elliptical adhesive contact. J. Appl. Phys. 107(11), 113512 (2010)

20. Jin, C., Jagota, A., Hui, C.: An easy-to-implement numerical simulation method for adhesive contact problems involving asymmetric adhesive contact. J. Phys. D Appl. Phys. 44(40), 405303 (2011)

21. Johnson, K.: Contact Mechanics. Cambridge University Press, Cambridge (1985)

22. Byrd, P., Friedman, M.: Handbook of Elliptic Integrals for Engineers and Scientists, 2nd edn. Springer-Verlag Berlin Heidelberg, Berlin (1971).

23. Israelachvili, J.N.: Intermolecular and Surface Forces, 3rd edn. Academic Press, Amsterdam (2011)

24. Bazrafshan, M. de Rooij, M. B., Valefi, M., Schipper, D.: Numerical method for the adhesive normal contact analysis based on a Dugdale approximation. Tribol. Int. 112, 117-128 (2017) 



\title{
Paper B
}

\section{Response to Dr Greenwood's comments on “Extending the double-Hertz model to allow modeling of an adhesive elliptical contact"}

\author{
N.H.M. Zini ${ }^{1,2,{ }^{*}}$, M.B. de Rooij ${ }^{1}$, M. Bazr Afshan Fadafan ${ }^{1}$, N. Ismail ${ }^{1,2}$, D.J. Schipper ${ }^{1}$ \\ ${ }^{1}$ Department of Surface Technology and Tribology, Faculty of Engineering Technology, \\ University of Twente, the Netherlands \\ ${ }^{2}$ Faculty of Mechanical Engineering, Universiti Teknikal Malaysia Melaka, Hang Tuah \\ Jaya, 76100 Durian Tunggal, Melaka, Malaysia
}

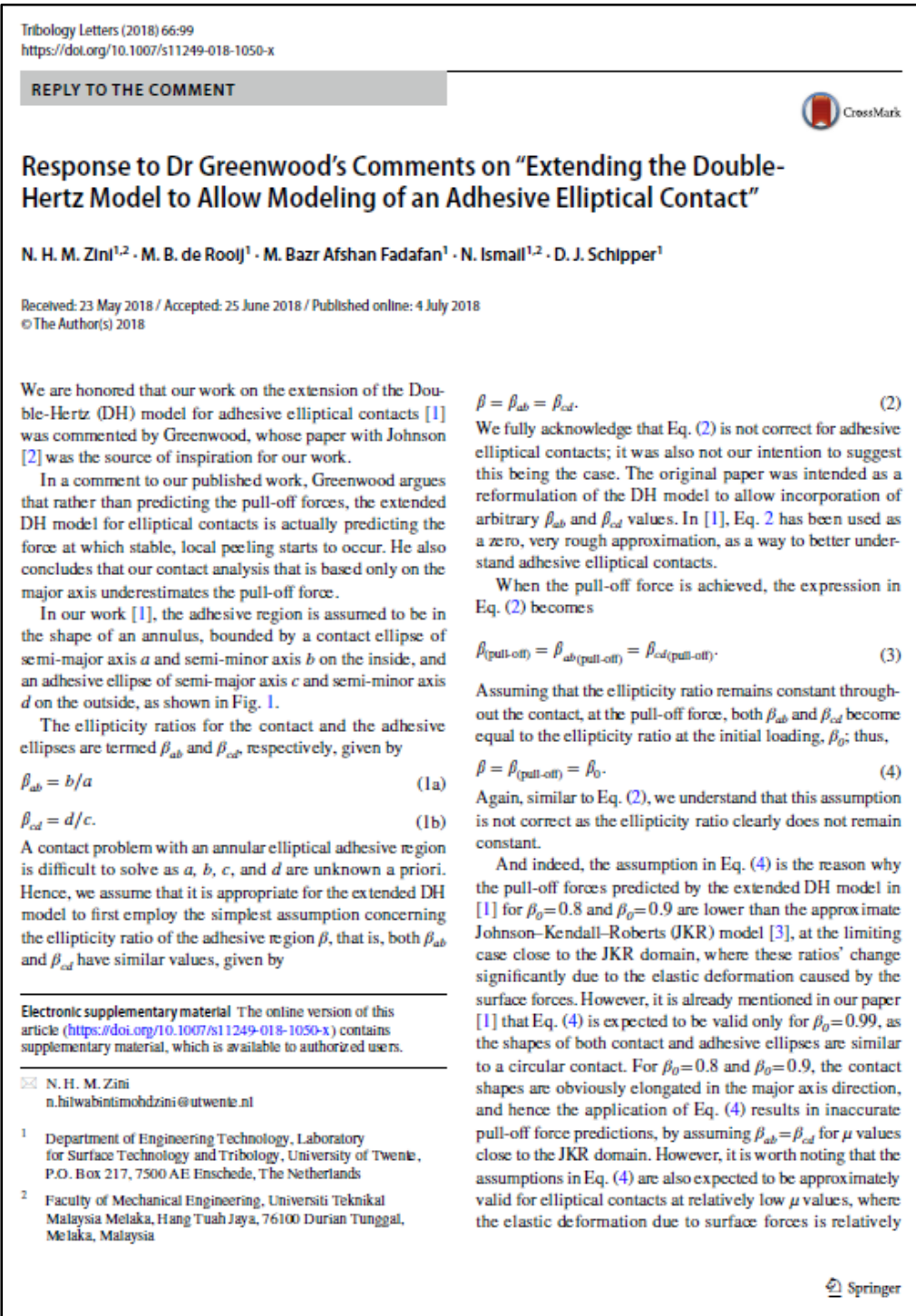



We are honored that our work on the extension of the Double-Hertz (DH) model for adhesive elliptical contacts [1] was commented by Greenwood, whose paper with Johnson [2] was the source of inspiration for our work.

In a comment to our published work, Greenwood argues that rather than predicting the pull-off forces, the extended $\mathrm{DH}$ model for elliptical contacts are actually predicting the force at which stable, local, peeling starts to occur. He also concludes that our contact analysis that is based only on the major axis underestimates the pull-off force.

In our work [1], the adhesive region is assumed to be in the shape of an annulus, bounded by a contact ellipse of semi-major axis $a$ and semi-minor axis $b$ on the inside, and an adhesive ellipse of semi-major axis $c$ and semi-minor axis $d$ on the outside, as shown in Fig. 1.

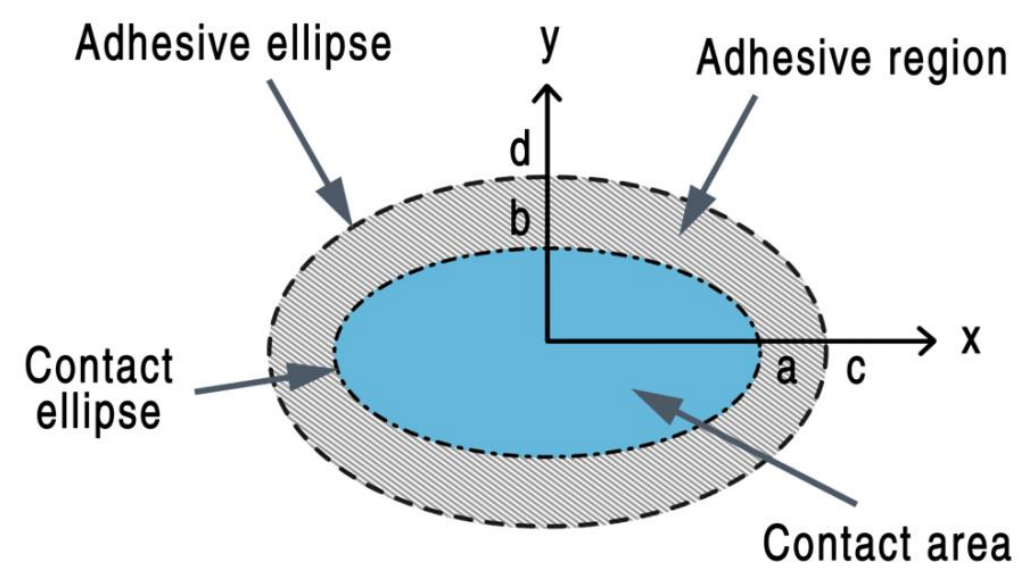

Fig. 1: Adhesive region of a $\mathrm{DH}$-based elliptical contact.

The ellipticity ratio for the contact and the adhesive ellipses are termed $\beta_{a b}$ and $\beta_{c d}$, respectively, given by:

$$
\begin{aligned}
& \beta_{a b}=b / a \\
& \beta_{c d}=d / c
\end{aligned}
$$

A contact problem with an annular elliptical adhesive region is difficult to solve as $a, b, c$ and $d$ are unknown a priori. Hence, we assume that it is appropriate for the extended $\mathrm{DH}$ model to first employs the simplest assumption concerning the ellipticity ratio of the adhesive region $\beta$, that is both $\beta_{a b}$ and $\beta_{c d}$ have similar values, given by:

$$
\beta=\beta_{a b}=\beta_{c d}
$$


We fully acknowledge that Eq. (2) is not correct for adhesive elliptical contacts; it was also not our intention to suggest this being the case. The original paper was intended as a reformulation of the $\mathrm{DH}$ model to allow incorporation of arbitrary $\beta_{a b}$ and $\beta_{c d}$ values. In [1], Eq. 2 has been used as a zero, very rough approximation, as a way to better understand adhesive elliptical contacts.

When the pull-off force is achieved, the expression in Eq. (2) becomes:

$$
\beta_{(p u l l-o f f)}=\beta_{a b_{(p u l l-o f f)}}=\beta_{c d(p u l l-o f f)}
$$

Assuming that the ellipticity ratio remains constant throughout the contact, at the pull-off moment, both $\beta_{a b}$ and $\beta_{c d}$ become equal to the ellipticity ratio at the initial loading, $\beta_{0}$, thus:

$$
\beta=\beta_{(\text {pull }-o f f)}=\beta_{0}
$$

Again, similar to Eq. (2), we understand that this assumption is not correct as the ellipticity ratio clearly does not remain constant.

And indeed, the assumption in Eq. (4) is the reason why the pull-off forces predicted by the extended $\mathrm{DH}$ model in [1] for $\beta_{0}=0.8$ and $\beta_{0}=0.9$ are lower than the approximate Johnson-Kendall-Roberts (JKR) model [3], at the limiting case close to the JKR domain, where these ratios' change significantly due to the elastic deformation caused by the surface forces. However, it is already mentioned in our paper [1] that Eq. (4) is expected to be valid only for $\beta_{0}=0.99$, as the shape of both contact and adhesive ellipses are similar to a circular contact. For $\beta_{0}=0.8$ and $\beta_{0}=0.9$, the contact shapes are obviously elongated in the major axis direction, hence the application of Eq. (4) results in inaccurate pull-off force predictions, by assuming $\beta_{a b}=\beta_{c d}$ for $\mu$ values close to the JKR domain. However, it is worth noting that the assumptions in Eq. (4) is also expected to be approximately valid for elliptical contacts at relatively low $\mu$ values, where the elastic deformation due to surface forces is relatively low. Although for increasing $\mu$ values, it is expected that the geometry of the adhesive contact will evolve from an elliptical geometry to a JKR-like geometry.

To show that the extended DH model framework [1] can predict accurately the pulloff force for various contact cases with the correct $\beta_{a b}$ and $\beta_{c d}$ assumptions, we simulate the adhesive elliptical contact using a Dugdale-based Boundary Element Model (BEM) that employs the Conjugate Gradient Method (CGM) [4]. The numerical simulation can be done for various contact cases, here the contact for $\beta_{0}$ values of $0.99,0.8$ and 0.5 is simulated, similar to the cases discussed in Greenwood's comment. The model is further explained in Supplementary Material. The obtained $\beta_{a b}, \beta_{c d}$ and $a$ values from the numerical simulations are then applied in the extended $\mathrm{DH}$ model to predict the pull-off forces. 
Pull-off force predictions from various models are compared in Table 1, for $\mu=1$. The table includes pull-off force predictions by the extended DH model and the numerical simulations, Greenwood's result which he applied a direct JKR solution for the extended DH model and also results using the approximate JKR model in [3]. When the pull-off force is achieved, the numerical simulations predict $\beta_{a b}$ and $\beta_{c d}$ to have different values from each other, higher than $\beta_{0}$ at the initial loading. It has to be noted that unlike the results in Greenwood's comment, here the pull-off forces are transformed into non-dimensional forms similar to [1], following the Derjaguin approximation for the case of an adhesive contact between two cylinders of radii $R_{1}$ and $R_{2}$, crossed at an angle, $\theta$ to each other [5]. Then, the applied force, $W$ can be expressed in a non-dimensional form as $W^{*}$, which is given as:

$$
W^{*}=\frac{W \sin \theta}{2 \pi R \Delta \gamma}
$$

$R$ is the relative radius and $\Delta y$ is the surface energy. From Table 1 , it is shown that the extended DH model predicts higher pull-off forces compared to the approximate JKR model [3] and the calculations by Greenwood. This situation is expected as the results from the extended DH model are obtained for low $\mu$ value of 1 , which is outside the JKR domain. Results in Table 1 show that with a proper assumption for the adhesive region (varying $\beta_{a b}$ and $\beta_{c d}$ throughout the contact), the extended $\mathrm{DH}$ model can indeed predict accurately the pull-off force for various cases of adhesive elliptical contacts.

Table 1: Comparison between pull-off force predictions for various elliptical contacts at $\mu=1$.

\begin{tabular}{|c|c|c|c|c|c|c|}
\hline \multirow[t]{2}{*}{$\begin{array}{l}\beta_{0}=b / a \\
(\text { Hertzian) }\end{array}$} & \multirow{2}{*}{$\begin{array}{l}\text { Direct } \\
K_{1} \\
\text { (Greenwood) } \\
W^{*}\end{array}$} & \multirow{2}{*}{$\begin{array}{l}\text { Approx. } \\
\text { JKR } \\
\text { model } \\
W^{*}\end{array}$} & \multicolumn{3}{|c|}{ Numerical model } & \multirow{2}{*}{$\begin{array}{l}\text { Extended } \\
\text { DH model } \\
W\end{array}$} \\
\hline & & & $\beta_{a b(\text { pull-off) }}$ & $\beta_{c d(\text { pull-off) }}$ & W & \\
\hline 0.99 & 0.7462 & 0.7500 & 0.9927 & 0.9943 & 0.7901 & 0.7941 \\
\hline 0.8 & 0.6584 & 0.7370 & 0.8232 & 0.8489 & 0.7785 & 0.7864 \\
\hline 0.5 & 0.4453 & 0.6366 & 0.5486 & 0.5982 & 0.6926 & 0.692 \\
\hline
\end{tabular}


Finally, it is worth to note that the purpose of our paper [1] is to present the development of an adhesive elliptical model, achieved by extending the $\mathrm{DH}$ theory. The model development is completed, and validated for a high $\beta_{0}$ value of 0.99 . For lower $\beta_{0}$ values, to assume constant ellipticity ratios for the adhesive region is indeed inaccurate, as also discussed in our paper [1]. We have shown through numerical simulations that both $\beta_{a b}$ and $\beta_{c d}$ do change significantly as the contact progresses. With this knowledge, we have continued the work on the extended $\mathrm{DH}$ model by finding the solutions for $\beta_{a b}$ and $\beta_{c d}$ that are suitable for a wide range of contact conditions. These solutions are already obtained and are planned to be published in a follow-up paper. By employing these solutions in the extended DH model, accurate prediction of adhesive elliptical contacts can be made for various contact conditions.

We again would like to thank Greenwood for his comments, which provide us an opportunity to elaborate more on our work.

\section{Acknowledgements}

The authors gratefully acknowledge support from the Ministry of Education Malaysia, Universiti Teknikal Malaysia Melaka and Green Tribology and Engine Performance (GTriboE) research group.

\section{References}

1. Zini, N.H.M., de Rooij, M.B., Bazr Afshan Fadafan, M. et al. Tribol. Lett. (2018) 66: 30. https://doi.org/10.1007/s11249-017-0976-8

2. Greenwood, J.A., Johnson, K.L: An alternative to the Maugis model of adhesion between elastic spheres. J. Phys. D Appl. Phys. 31(22), 3279-3290 (1998)

3. Johnson, K.L., Greenwood, J.A.: An approximate JKR theory for elliptical contacts. J. Phys. D Appl. Phys. 38(7), 1042-1046 (2005)

4. Bazrafshan, M. de Rooij, M.B., Valefi, M., Schipper, D.J.: Numerical method for the adhesive normal contact analysis based on a Dugdale approximation. Tribol. Int. 112, 117-128 (2017)

5. Israelachvili, J.N.: Intermolecular and Surface Forces, 3rd edn. Academic Press, Amsterdam (2011)

6. Maugis, D.: Adhesion of spheres: The JKR-DMT transition using a Dugdale model. J. Colloid Interface Sci. 150(1), 243-269 (1992) 


\title{
Paper C
}

\section{Predicting the pull-off force of the double-Hertz elliptical contact with a load-dependent adhesive region}

\author{
N.H.M. Zini ${ }^{12^{*}}$, M.B. de Rooij ${ }^{1}$, M. Bazrafshan ${ }^{1}$, A. Akchurin ${ }^{1}$, D.J. Schipper ${ }^{1}$ \\ 1) Department of Surface Technology and Tribology, Faculty of Engineering Technology, \\ University of Twente, the Netherlands \\ 2) Faculty of Mechanical Engineering, Universiti Teknikal Malaysia Melaka, \\ Hang Tuah Jaya, 76100 Durian Tunggal, Melaka, Malaysia
}

Submitted to Tribology Transactions 

Abstract Adhesive elliptical contacts are found in small-scale applications such as fiberon-fiber contacts. A recently extended Double-Hertz $(\mathrm{DH})$ model for adhesive elliptical contacts is shown to predict accurately the pull-off force, but the employed assumptions restricted the model application to nearly circular contacts. Ellipticity ratios of the inner and the outer boundaries of a $\mathrm{DH}$ based adhesive region cannot be determined as the geometry of the adhesive region is unknown a priori, thus making the model unsuitable for a wide range of elliptical contacts. Here, the equations at the pull-off moment for the boundaries of the adhesive region and the semi-major axis of the elliptical contact area are proposed and incorporated into the extended $\mathrm{DH}$ model for various elliptical contacts. Initially, the geometry of the adhesive region at the pull-off moment is predicted using numerical simulations utilizing the Boundary Element Method. The numerical data is used to curve-fit equations of the ellipticity ratio and the semi-major axis of the elliptical contact area. The resulting equations are then incorporated into the extended $\mathrm{DH}$ model for pull-off force predictions. Results show that the incorporated equations allow the extended $\mathrm{DH}$ model to predict the pull-off force for various elliptical contacts within its working domain. For adhesive elliptical contacts, the limit of the JKR domain is also shown to be shifted closer to the DMT limit, expanding the validity domain of the JKR model to lower values of the Tabor parameter.

Keywords Double-Hertz model; elliptical contact; pull-off force; adhesion

\section{List of symbols}

$a, b$

$c, d$

$\beta_{\text {Hertz }}$

$\beta_{0}$

$\beta_{a b}$

$\beta_{c d}$

$r$

$\theta_{p}$

$r_{a b}$

$r_{c d}$

$\theta_{\text {skew }}$
Semi-major and semi-minor axes of the contact ellipse at the pull-off moment

Semi-major and semi-minor axes of the adhesive ellipse at the pull-off moment

Ellipticity ratio of the Hertzian contact

Ellipticity ratio of the initial contact

Ellipticity ratio of the contact ellipse at the pull-off moment

Ellipticity ratio of the adhesive ellipse at the pull-off moment

Radial coordinate

Angular coordinate

Radial coordinate of the contact ellipse

Radial coordinate of the adhesive ellipse

Angle between two cylindrical bodies 
$E^{*}$

$R=\sqrt{R^{\prime} R^{\prime \prime}}$

$\mu$

$k_{a b}=\left(1-\beta_{a b}{ }^{2}\right)^{1 / 2}$

$k_{c d}=\left(1-\beta_{c d}^{2}\right)^{1 / 2}$

$k_{a b}{ }^{\prime}=\left(1-k_{a b}{ }^{2}\right)^{1 / 2}$

$\varphi=\sin ^{-1}\left(a / \sqrt{l+a^{2}}\right)$

$\boldsymbol{K}(k)$

$\boldsymbol{E}(k)$

$\boldsymbol{F}(\varphi, k)$

$\boldsymbol{E}(\varphi, k)$

$\Delta \gamma$

$v$

$W$

$P o_{a b}$

$\mathrm{Po}_{c d}$

$\omega_{i n_{a b}}$

$\omega_{\text {out }}$ ab

$\omega_{i n_{c d}}$

$\omega_{0 a b}$

$\omega_{a b c d}$

$z_{0}$

$\left.h_{0}=0.974 z_{0}\right)$

$\sigma_{0}=16 \Delta \gamma / 9 \sqrt{3} z_{0}$

$P(x, y)$

$g(x, y)$

$F_{0}$
Reduced Young's modulus

Equivalent radius

Tabor parameter

Elliptic modulus of the contact ellipse at the pull-off moment

Elliptic modulus of the adhesive ellipse at the pull-off moment

Complementary elliptic modulus of the contact ellipse at the pull-off moment

Second argument of the incomplete elliptic integrals at the pulloff moment

Complete elliptic integral of the first kind

Complete elliptic integral of the second kind

Incomplete elliptic integral of the first kind

Incomplete elliptic integral of the second kind

Surface energy

Scaling factor to keep $\sigma_{0}$ and $\Delta y$ at a constant value

Applied load of the single asperity contact

Maximum pressure of the contact ellipse

Maximum pressure of the adhesive ellipse

Surface displacement within the contact ellipse

Surface displacement outside the contact ellipse

Surface displacement within the adhesive ellipse

Combined surface displacement for $0 \leq r \leq r_{a b}$

Combined surface displacement for $r_{a b} \leq r \leq r_{c d}$

Equilibrium separation

Separation limit between contacting surfaces for the MD model Maximum adhesive stress for the MD model

Applied pressure for the MD model

Separation between two surfaces after deformation, for the MD model

Applied load for the MD model 


\section{Introduction}

Contact modeling is essential in micro-scale applications such as fiber-on-fiber contacts. At this scale, adhesion typically plays a major role in contact deformations as a consequence of high surface area-to-volume ratios [1]. For a simple contact behavior like adhesive circular contacts, there are several contact models available such as the Derjaguin-Muller-Toporov (DMT) model [2], which was later corrected by Muller et al. [3], the Johnson-Kendall-Roberts (JKR) model [4], the Maugis-Dugdale (MD) cohesive model [5] and the Double-Hertz (DH) model [6].

An adhesion map for circular contacts by Johnson and Greenwood [7] shows the application range of the adhesive models. The Tabor parameter, $\mu$ developed in [8], calculates the ratio of the neck height to the equilibrium separation. For large values of $\mu$, adhesion is limited to the contact area. Thus, the adhesive stresses outside the contact area can be disregarded as the surfaces are totally separated outside the contact area, which is the basis of a JKR contact [4]. On the other hand, in the case of the DMT model, the presence of adhesion is significant outside the contact area. No deformation is allowed in the DMT model, hence no neck is formed outside the contact area. The DMT contact follows a continuous and stable path until a separation occurs at zero contact as opposed to the separation at a finite value of the contact radius for the JKR model $[4,9,10]$. This contact behavior is similar to the Hertzian contact, though the DMT contact is separated at the pull-off force value rather than at zero load [2]. For the MD model in [5], the adhesive stresses are constant within the adhesive annular region. Occupying the intermediate zone in the adhesion map, the MD model smoothly reconciles the difference between the DMT and the JKR models. Another intermediate model, the $\mathrm{DH}$ model [6], is not part of the adhesion map [7]. The contact geometry of the DH model is similar to the MD model [5], though the $\mathrm{DH}$ model is simpler in mathematical formulations [6]. For the $\mathrm{DH}$ model, adhesion is modeled by the difference between two Hertzian pressure distributions. These pressure distributions are the results of two Hertzian circular contact areas of different radii. The adhesive region outside the contact is obtained by the area difference between the two Hertzian contact areas. All the adhesive models mentioned above have been extended to allow predictions for adhesive line contacts. However, for more complicated surface interactions such as adhesive elliptical contacts, there is no model available between the DMT and the JKR domains.

The approximate JKR model developed in [11] has provided the knowledge on how an adhesive elliptical contact behaves. This model has been developed on the basis that the contact area remains elliptical, with both major and minor axes having the same stress 
intensity factor. The resulting eccentricity due to the ellipticity ratio of the contact area is shown to vary with load. The approximate JKR model does give good results, though it is only valid for highly elastic materials. A comparison with experimental results of Polydimethylsiloxane (PDMS) material in [12] shows that both the approximate JKR model and the experiment have similar pull-off force values; the relative errors between both results only exceed $10 \%$ when the skew angle between the surfaces, $\theta_{\text {skew }}$ decreases to $20^{\circ}$ and less. As $\theta_{\text {skew }}$ approaches $0^{\circ}$, the contact areas obtained from the experiment start to detach in the major axis direction, which is not predicted by the approximate JKR model [12]. A numerical simulation in [13] also compares its results to the approximate JKR model in [11] and the experiments in [12], which show that the numerical simulation has closer results to the approximate JKR model at lower $\theta_{\text {skew }}$ values. Recently, the approximate JKR model has been modified in [14], to generalize the contact prediction by assuming that the contact areas for both adhesive and non-adhesive contacts are similar, with a suitable increased load.

Another adhesive elliptical model is proposed in [15] and is used to describe the adhesive elliptical contacts between rigid filaments. The DMT approach from [2] is employed to calculate the contact geometry and the elastic deformations, while Bradley's theory in [16] is used in the DMT-based model to predict adhesion. As $\theta_{\text {skew }}$ between two filaments increases, the contact area begins to shrink rapidly as the filament radius increases. Unlike the JKR model in [11], the pull-off force of the DMT elliptical model in [15] is independent of the size of the contact area.

The limited application of the JKR and the DMT elliptical models are solved by the introduction of an adhesive elliptical model that employs the $\mathrm{DH}$ theory [17], which assumptions are clarified further in [18]. Pull-off force predictions are shown to be underestimated due to the assumption that both inner and outer boundaries of the adhesive region have constant, identical ellipticity ratios from the initial load to the pull-off force at the pull-off moment, following the Hertzian assumption for non-adhesive contacts. Results of the extended $\mathrm{DH}$ model in the JKR domain are closer to the approximate JKR model [11] by using the geometrical properties from the JKR model that vary with load, but not close enough as both boundaries of the adhesive region are assumed to have similar ellipticity ratios. Based on this observation, it is concluded that an accurate modeling of an adhesive elliptical contact can be made possible for the extended DH model in [17] by modeling an adhesive region with load-dependent boundaries that change throughout the contact. 
For the current research, the adhesive region is allowed to change without the constraint of a Hertzian contact. Both contact and adhesive ellipses that act as the inner and the outer boundaries of the adhesive region have ellipticity ratios that vary with load, with both ellipses having similar ellipticity ratios at the initial load. As the contact progresses to the pull-off moment where the 'negative' separation force is maximum (the pull-off force), both boundaries begin to change in size, with the inner boundary having a different ellipticity ratio than the outer boundary, though both boundaries are assumed to keep their elliptical shapes. The work consists of two parts: (1) predicting the geometry of the adhesive region at the pull-off moment by using a numerical model; and (2) developing equations to predict the ellipticity ratio of the inner and the outer boundaries of the adhesive region, and also the semi-major axis of the elliptical contact area, both at the pull-off moment. Results obtained will be compared with the numerical model, other adhesive elliptical contact models and existing experimental results for validation purposes. It will also be analysed if the application limit for the JKR model still applies for adhesive elliptical contacts.

\section{Load-dependence of DH-based elliptical contacts}

\subsection{Adhesion modeling}

Continuing the work in [6], the behavior of an adhesive elliptical contact is modeled using the $\mathrm{DH}$ approach in [17], using the polar coordinate system whose geometrical parameters are defined in Appendix A. Using the DH approach, adhesion is modeled by the difference between two Hertzian pressure distributions. Both Hertzian pressures are assumed to have equivalent shapes, the only difference being the size of the area of which the pressure acts. The smaller ellipse acts as the inner boundary of the adhesive region, while the larger ellipse acts as the outer boundary (termed respectively as the contact and the adhesive ellipses), resulting in an annular region where the adhesion forces act, as shown in Fig. 1. The contact ellipse is the Hertzian contact area, with semi-major axis a and semiminor axis $b$. The adhesive ellipse is due to the additional pressure distribution outside the contact ellipse, with semi-major axis $c$ and semi-minor axis $d$. 


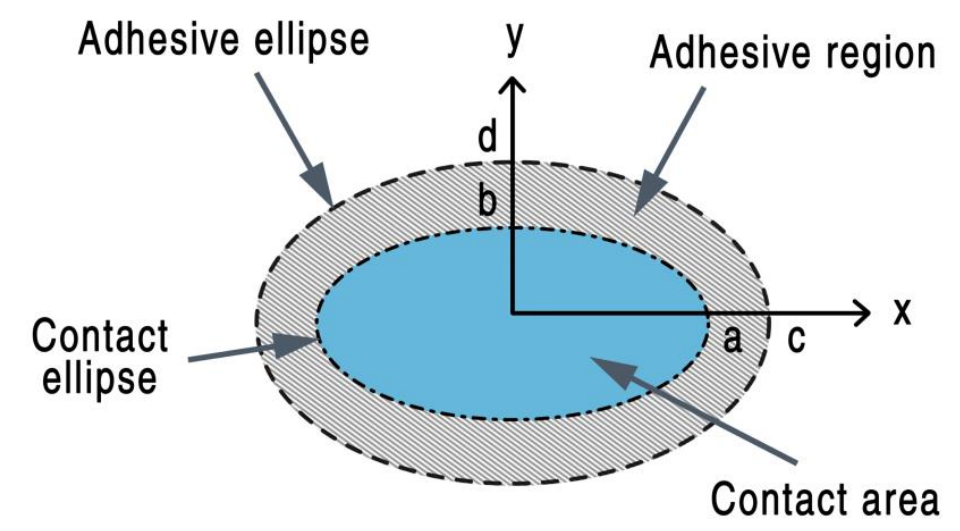

Fig. 1: Adhesive region of a DH-based elliptical contact [18].

With the new assumption of $\mathrm{DH}$-based elliptical contacts with adhesive region boundaries that vary with load, selected adhesive equations are reproduced in this paper, focusing on adhesive contacts at the pull-off moment. Based on the extended DH model in [17], the adhesive stresses for DH based elliptical contacts can be expressed in polar coordinates as:

$P\left(r, \theta_{p}\right)=$

$$
v\left\{P o_{c d}\left[1-\left(\frac{r \cos \theta_{p}}{c}\right)^{2}-\left(\frac{r \sin \theta_{p}}{d}\right)^{2}\right]^{1 / 2}-P o_{a b}\left[1-\left(\frac{r \cos \theta_{p}}{a}\right)^{2}-\left(\frac{r \sin \theta_{p}}{b}\right)^{2}\right]^{1 / 2}\right\} \quad 0 \leq r \leq r_{a b}
$$

$$
P\left(r, \theta_{p}\right)=v P o_{c d}\left[1-\left(\frac{r \cos \theta_{p}}{c}\right)^{2}-\left(\frac{r \sin \theta_{p}}{d}\right)^{2}\right]^{1 / 2} \quad r_{a b} \leq r \leq r_{c d}
$$

where $v$ is the scaling factor, $r$ is the radial coordinate and $\theta_{p}$ is the angular coordinate of the selected point in the contact region. $r_{a b}$ and $r_{c d}$ are the radial coordinates for the contact and the adhesive ellipses respectively, such that $r_{a b}<r_{c d}$. The result of the adhesive stresses distribution in [17] shows that within the adhesive region, the maximum adhesive stresses occurs at $r_{a b}$ and decreases to zero value at $r_{c d}$. 
The maximum pressure in the contact and the adhesive ellipses, $P o_{a b}$ and $P o_{c d}$ can be expressed as:

$$
\begin{aligned}
P o_{a b} & =\frac{E^{*}}{2 R} \frac{k_{a b}{ }^{2} a}{\beta_{a b}\left\{\left[\boldsymbol{E}\left(k_{a b}\right) / \beta_{a b}{ }^{2}-\boldsymbol{K}\left(k_{a b}\right)\right]\left[\boldsymbol{K}\left(k_{a b}\right)-\boldsymbol{E}\left(k_{a b}\right)\right]\right\}^{1 / 2}} \\
P o_{c d} & =\frac{E^{*}}{2 R} \frac{k_{c d}{ }^{2} c}{\beta_{c d}\left\{\left[\boldsymbol{E}\left(k_{c d}\right) / \beta_{c d}{ }^{2}-\boldsymbol{K}\left(k_{c d}\right)\right]\left[\boldsymbol{K}\left(k_{c d}\right)-\boldsymbol{E}\left(k_{c d}\right)\right]\right\}^{1 / 2}}
\end{aligned}
$$

where $\boldsymbol{K}(k)$ and $\boldsymbol{E}(k)$ are the complete elliptic integral of the first and the second kind, and $k$ is the elliptic modulus (eccentricity of the ellipse). Unlike in [17], due to the new assumption of a load-dependent adhesive region, $k$ values for the contact and the adhesive ellipses in this paper are not equal, hence the different definitions of $k_{a b}$ and $k_{c d}$. As this paper focuses on the elliptical contacts at the pull-off moment, $\beta_{a b}$ and $\beta_{c d}$ are given as:

$$
\begin{aligned}
& \beta_{a b}=\beta_{a b_{\text {pull-off }}} \\
& \beta_{c d}=\beta_{c d_{\text {pull-off }}}
\end{aligned}
$$

Based from the results in [17], for a load-dependent adhesive region, at the pull-off moment, the relation between the initial ellipticity ratio at the beginning of the adhesive contact, $\beta_{0}, \beta_{a b}$ and $\beta_{c d}$ can be described as:

$$
\beta_{0} \neq \beta_{a b} \neq \beta_{c d}
$$

$\beta_{\text {Hertz }}$ is introduced to describe the constant ellipticity ratio obtained from a normal Hertzian elliptical contact. Before the ellipticity ratios change due to the applied load, the assumption for the initial adhesive contact for the contact and the adhesive ellipses can be summarized as:

$$
\begin{gathered}
\beta_{0}=\beta_{0_{a b}}=\beta_{0_{c d}} \\
\beta_{0}=\beta_{\text {Hertz }}
\end{gathered}
$$

which the relation between $\beta_{\text {Hertz }}$ to $\theta_{\text {skew }}$ is solved in [10].

The surface displacement between two cylindrical bodies, $\omega$, is given by:

$$
\begin{array}{cl}
\omega_{0 a b}\left(r, \theta_{p}\right)=v\left(\omega_{i n_{a b}}-\omega_{i n_{c d}}\right) & 0 \leq r \leq r_{a b} \\
\omega_{a b c d}\left(r, \theta_{p}\right)=v\left(\omega_{o u t_{a b}}-\omega_{i n_{c d}}\right) & r_{a b} \leq r \leq r_{c d}
\end{array}
$$

where

$$
\begin{aligned}
& \omega_{i n_{a b}}\left(r, \theta_{p}\right)=\frac{1}{\pi E^{*}}\left[L_{i n_{a b}}-M_{\text {in }_{a b}}\left(r \cos \theta_{p}\right)^{2}-N_{\text {in }_{a b}}\left(r \sin \theta_{p}\right)^{2}\right] \quad 0 \leq r \leq r_{a b} \\
& \omega_{\text {out }_{a b}}\left(r, \theta_{p}\right)=\frac{1}{\pi E^{*}}\left[L_{\text {out }_{a b}}-M_{\text {out }_{a b}}\left(r \cos \theta_{p}\right)^{2}-N_{\text {out }_{a b}}\left(r \sin \theta_{p}\right)^{2}\right] \quad r \geq r_{a b}
\end{aligned}
$$

and

$$
\omega_{i n_{c d}}\left(r, \theta_{p}\right)=\frac{1}{\pi E^{*}}\left[L_{i n_{c d}}-M_{i n_{c d}}\left(r \cos \theta_{p}\right)^{2}-N_{i n_{c d}}\left(r \sin \theta_{p}\right)^{2}\right] \quad 0 \leq r \leq r_{c d}(8)
$$


Definitions of $L, M$ and $N$ can be found in Appendix B.

From [14], the combined load for adhesive elliptical contacts is obtained by integrating the stresses over the elliptical contact area and the annulus adhesive region, which can be expressed as:

$$
W=\frac{4 E^{*}}{3 R}\left\{\frac{(a b)^{3 / 2}}{F_{a b}{ }^{3}}-v\left[\frac{(c d)^{3 / 2}}{F_{c d^{3}}}-\frac{(a b)^{3 / 2}}{F_{a b^{3}}}\right]\right\}
$$

where

$$
\begin{aligned}
F_{a b} & =\left(\frac{4}{\pi k_{a b}^{2}} \beta_{a b}^{3 / 2}\left\{\left[\boldsymbol{E}\left(k_{a b}\right) / \beta_{a b}^{2}-\boldsymbol{K}\left(k_{a b}\right)\right]\left[\boldsymbol{K}\left(k_{a b}\right)-\boldsymbol{E}\left(k_{a b}\right)\right]\right\}^{1 / 2}\right)^{1 / 3} \\
F_{c d} & =\left(\frac{4}{\pi k_{c d}^{2}} \beta_{c d}^{3 / 2}\left\{\left[\boldsymbol{E}\left(k_{c d}\right) / \beta_{c d}^{2}-\boldsymbol{K}\left(k_{c d}\right)\right]\left[\boldsymbol{K}\left(k_{c d}\right)-\boldsymbol{E}\left(k_{c d}\right)\right]\right\}^{1 / 2}\right)^{1 / 3}
\end{aligned}
$$

Following [6], results are summarized in non-dimensional form, with the geometry of the adhesive region at the pull-off moment given as:

$$
a^{*}=a / c_{r} ; b^{*}=b / c_{r} ; c^{*}=c / c_{r} ; d^{*}=d / c_{r}
$$

where

$$
\begin{gathered}
c_{r}{ }^{3}=R^{2} \Delta \gamma / E^{*} \\
W^{*}=W \sin \theta_{\text {skew }} / 2 \pi R \Delta \gamma
\end{gathered}
$$

The load in Eq. (9) can be transformed into non-dimensional form as:

$$
W^{*}=\frac{2 \sin \theta_{\text {skew }}}{3 \pi}\left\{\frac{\left(a^{*} b^{*}\right)^{3 / 2}}{F_{a b}}-v\left[\frac{\left(c^{*} d^{*}\right)^{3 / 2}}{F_{c d}{ }^{3}}-\frac{\left(a^{*} b^{*}\right)^{3 / 2}}{F_{a b} b^{3}}\right]\right\}
$$

\subsection{Prediction of the adhesive region boundaries}

\subsubsection{Numerical approach}

For accurate behavior prediction of an adhesive elliptical contact at the pull-off moment, the model must employ a realistic and accurate assumption of the geometry of the contact and the adhesive ellipses, which determines both $\beta_{a b}$ and $\beta_{c d}$ values. The load effect on both $\beta_{a b}$ and $\beta_{c d}$ values during contact is recognized in [17] as the correct geometrical behavior for the $\mathrm{DH}$ based adhesive region. Results from [17] prove that with correct $\beta_{a b}$ and $\beta_{c d}$ values, it is possible for the extended $\mathrm{DH}$ model to predict accurately the pull-off force for various elliptical contacts. However, without the geometrical values of $a, b, c$ and $d$, both $\beta_{a b}$ and $\beta_{c d}$ cannot be solved. Here, numerical simulations will be utilized to predict accurately $\beta_{a b}$ and $\beta_{c d}$ values, for various elliptical contacts.

A Boundary Element Model (BEM) was developed in [19] to predict adhesion between rough surfaces, based on the Dugdale approximation for the adhesive stresses. 
From the MD model in [5], adhesion depends on the separation limit between the surfaces in contact, $h_{0}$. The relation between $h_{0}$ and the surface energy, $\Delta y$, is given as:

$$
\Delta \gamma=\sigma_{0} h_{0}
$$

$\sigma_{0}$ is the maximum adhesive stress, expressed as:

$$
\sigma_{0}=\frac{16 \Delta \gamma}{9 \sqrt{3} z_{0}}
$$

$z_{0}$ is the equilibrium separation. Using Eq. (16), Eq. (15) is rewritten as:

$$
h_{0}=\frac{9 \sqrt{3} z_{0}}{16}=0.974 z_{0}
$$

The separation between the surfaces, $\epsilon$ is given as:

$$
\epsilon= \begin{cases}0 & \text { at } r=a \\ h_{0} \text { at } r & =c\end{cases}
$$

Hence, the cohesive zone for the numerical model starts at the beginning of the constant pressure and ends when $\epsilon=h_{0}$.

From the theory in [5], a general adhesive contact problem can be described as having two surfaces deformed due to the applied pressure, $P(x, y)$ :

$$
\begin{gathered}
P(x, y)>-\sigma_{0} \quad \text { at } \quad g(x, y)=0 \\
P(x, y)=-\sigma_{0} \quad \text { at } \quad 0<g(x, y)<h_{0} \\
P(x, y)=0 \quad \text { at } \quad g(x, y)>h_{0} \\
\int P(x, y) d x d y=F_{0}
\end{gathered}
$$

$g(x, y)$ is the separation between two surfaces after deformation and $F_{0}$ is the applied load.

The BEM model in [19] utilizes an extended Conjugate Gradient Method (CGM), an iterative method that can solve non-linear optimization problems. The contact problem in Eq. (19) can be expressed in the discretized format as:

$$
\begin{aligned}
& P_{i j}>-\sigma_{0} \quad \text { at } \quad g_{i j}=0 \\
& P_{i j}=-\sigma_{0} \quad \text { at } \quad 0<g_{i j}<h_{0} \\
& P_{i j}=0 \quad \text { at } \quad g_{i j}>h_{0} \\
& \Delta x \Delta y \sum_{k=1}^{N} \sum_{j=1}^{N} P_{i j}=F_{0}
\end{aligned}
$$

where $i, j=1,2, \ldots, N$, which $N$ is the number of rectangular surface elements of the calculation area; a higher $N$ will produce a finer mesh.

CGM algorithm can solve complex contact problems, including adhesive elliptical contacts with a wide range of ellipticity ratios. In this algorithm, the geometry is not assumed as the contribution of each element in the pressure and the deformation profiles is considered separately [19]. Hence the algorithm can be used for any type of contact geometry including adhesive elliptical contacts. The CGM algorithm for solving the adhesive elliptical contacts is shown schematically in Fig. 2. 


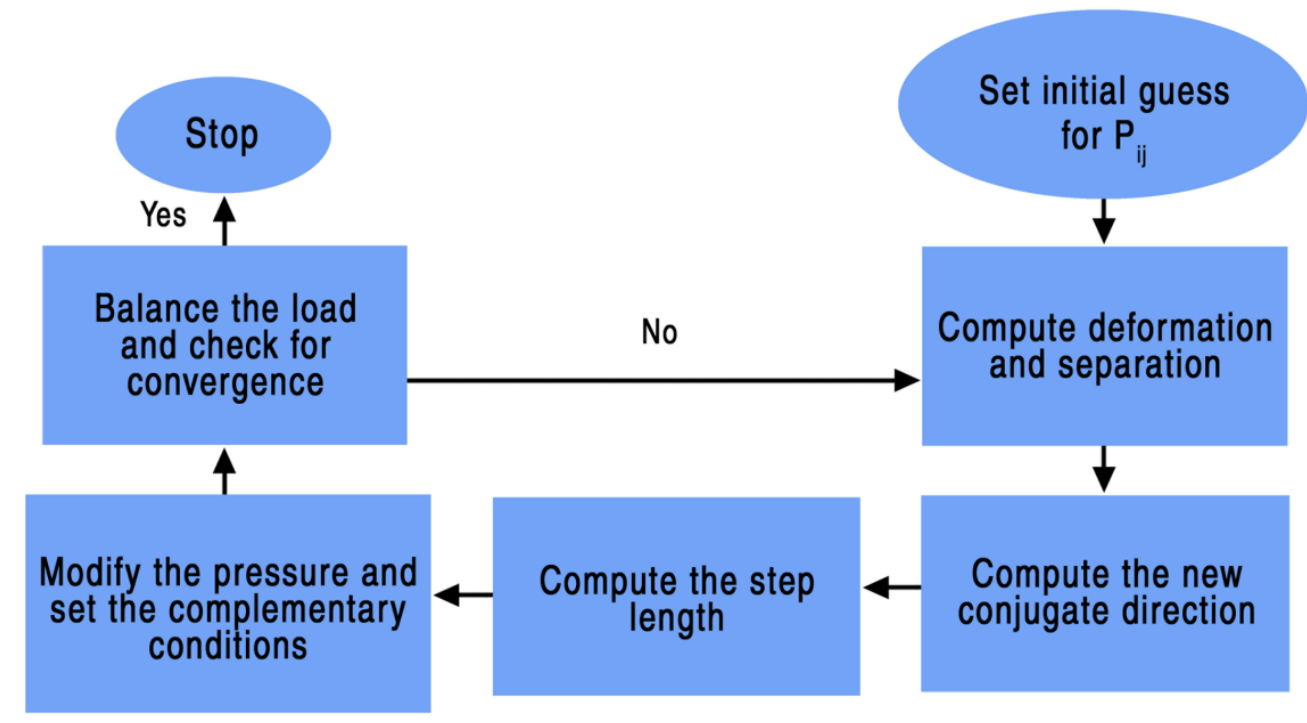

Fig. 2: CGM based algorithm for adhesive elliptical contacts [19].

Numerical simulations are conducted for $\beta_{0}$ values of $0.3\left(\theta_{\text {skew }}=43.63^{\circ}\right), 0.4\left(\theta_{\text {skew }}\right.$ $\left.=53.14^{\circ}\right), 0.6\left(\theta_{\text {skew }}=68.51^{\circ}\right)$ and $0.8\left(\theta_{\text {skew }}=80.45^{\circ}\right)$, within the range of $0.5 \leq \mu \leq 4$. Results from the numerical simulations are obtained using the element number, $N$ along the major and the minor axes of $N=2048$ for $0.5 \leq \mu<2$ (the domain size is 2048 elements $\times 2048$ elements) and $N=4096$ for $2 \leq \mu \leq 4$ (the domain size is 4096 elements $\mathrm{x}$ 4096 elements). In all cases, the calculation domain is set six times greater than the contact domain. An increased resolution is required for larger $\mu$ values due to the small adhesive zone outside the contact. These values are set after repeating the numerical computations using various element numbers and extension factors for the contact domain to get the optimal numerical parameters for all contact conditions. The accurate prediction of $\beta_{a b}$ and $\beta_{c d}$ values at the pull-off moment uses a negative load input in the numerical algorithm; this negative load represents the pull-off force required to separate the surfaces. The pull-off force is determined by the greatest negative load that first converges within 200 iterations.

An example of the numerical model's results on predicting adhesive elliptical contacts at the pull-off moment are shown in Fig. 3. The results are obtained using the contact parameters in Table 1 as the input. Fig. 3a shows the image of three-dimensional pressure profile within the computation domain with 2048 elements along the major and the minor axes while Fig. $3 \mathrm{~b}$ and Fig. $3 \mathrm{c}$ show the resulting pressure distribution along the semi-major axis and semi-minor axis. The selected points of $a$ and $b$ are obtained from the semi-major and semi-minor axes of the contact ellipse while the points of $c$ and $d$ are obtained from the semi-major and semi-minor axes of the adhesive ellipse. 
Table 1: Parameter of the adhesive elliptical contact at the pull-off moment.

\begin{tabular}{|l|l|l|}
\hline Parameters & Values & Unit \\
\hline Predicted pull-off force & 75 & $\mathrm{nN}$ \\
\hline Fiber diameter & 3.5 & $\mathrm{Mm}$ \\
\hline Skew angle, $\theta_{\text {skew }}$ & 43.63 & $\circ$ \\
\hline Hertzian ellipticity ratio, $\beta_{0}$ & 0.3 & - \\
\hline Tabor parameter, $\mu$ & 0.5 & - \\
\hline Number of elements along major and minor axes, $N$ & 2048 & - \\
\hline
\end{tabular}

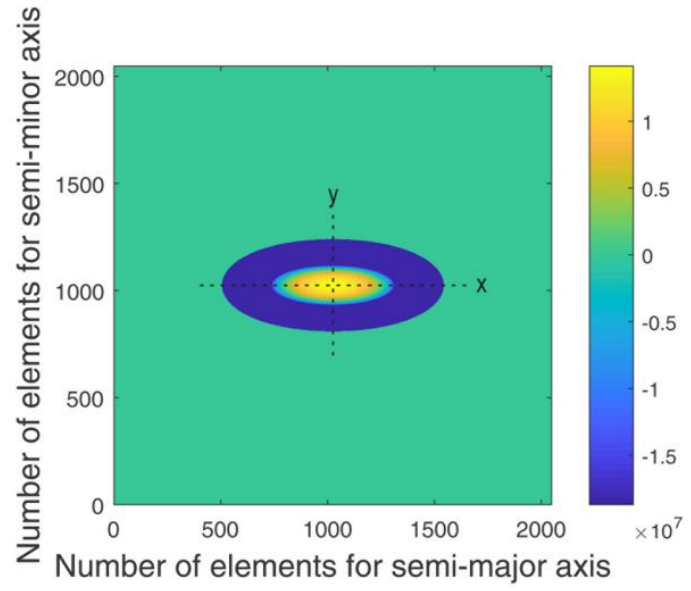

(a)

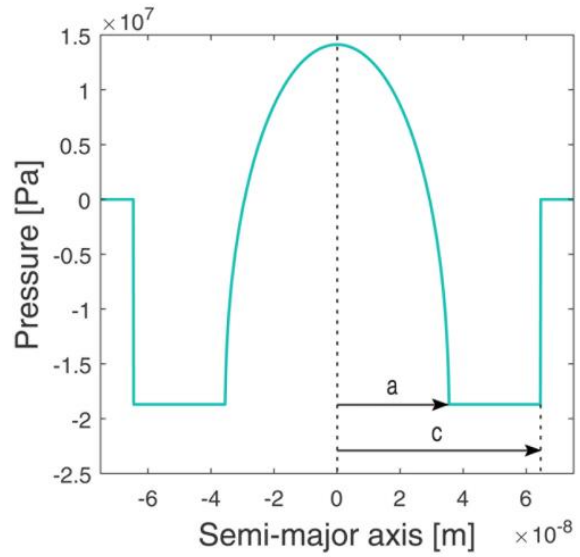

(b)

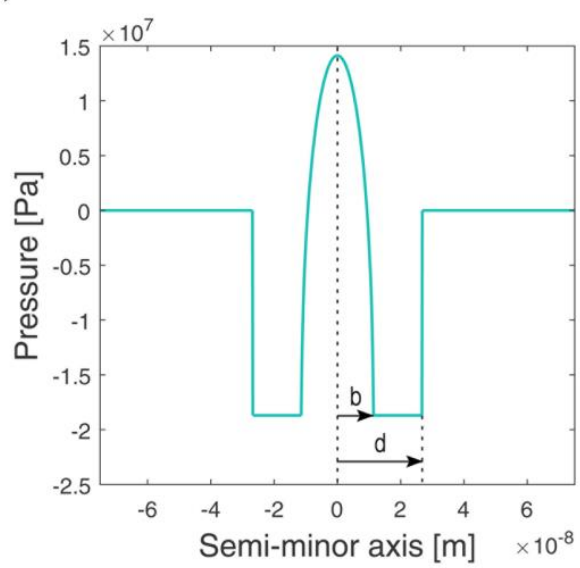

(c)

Fig. 3: Numerical solution for the elliptical contact (a) three-dimensional pressure profile for the elliptical contact (b) stress distribution along the semi-major axis and (c) stress distribution along the semi-minor axis. 
Next, the values of $\beta_{a b}$ and $\beta_{c d}$ are determined by applying the values of $a, b, c$ and $d$ obtained from numerical simulations into the ellipticity ratio equation from [17], given as:

$$
\begin{aligned}
& \beta_{a b}=b / a \\
& \beta_{c d}=d / c
\end{aligned}
$$

Fig. 4 shows the results from the numerical simulations for both contact and adhesive ellipses at the pull-off moment, for the effect of $\mu$ on the ellipticity ratio. Starting from $\mu=0.5$, the large differences between $\beta_{a b}$ and $\beta_{c d}$ become smaller as the $\mu$ values approach 3. It is also shown that both $\beta_{a b}$ and $\beta_{c d}$ at the pull-off moment are higher than $\beta_{0}$. This indicates that both ellipses transform from an elliptical shape at the beginning of the contact to a much rounder ellipse at the pull-off moment, a behavior also reported in $[11,12,20]$.
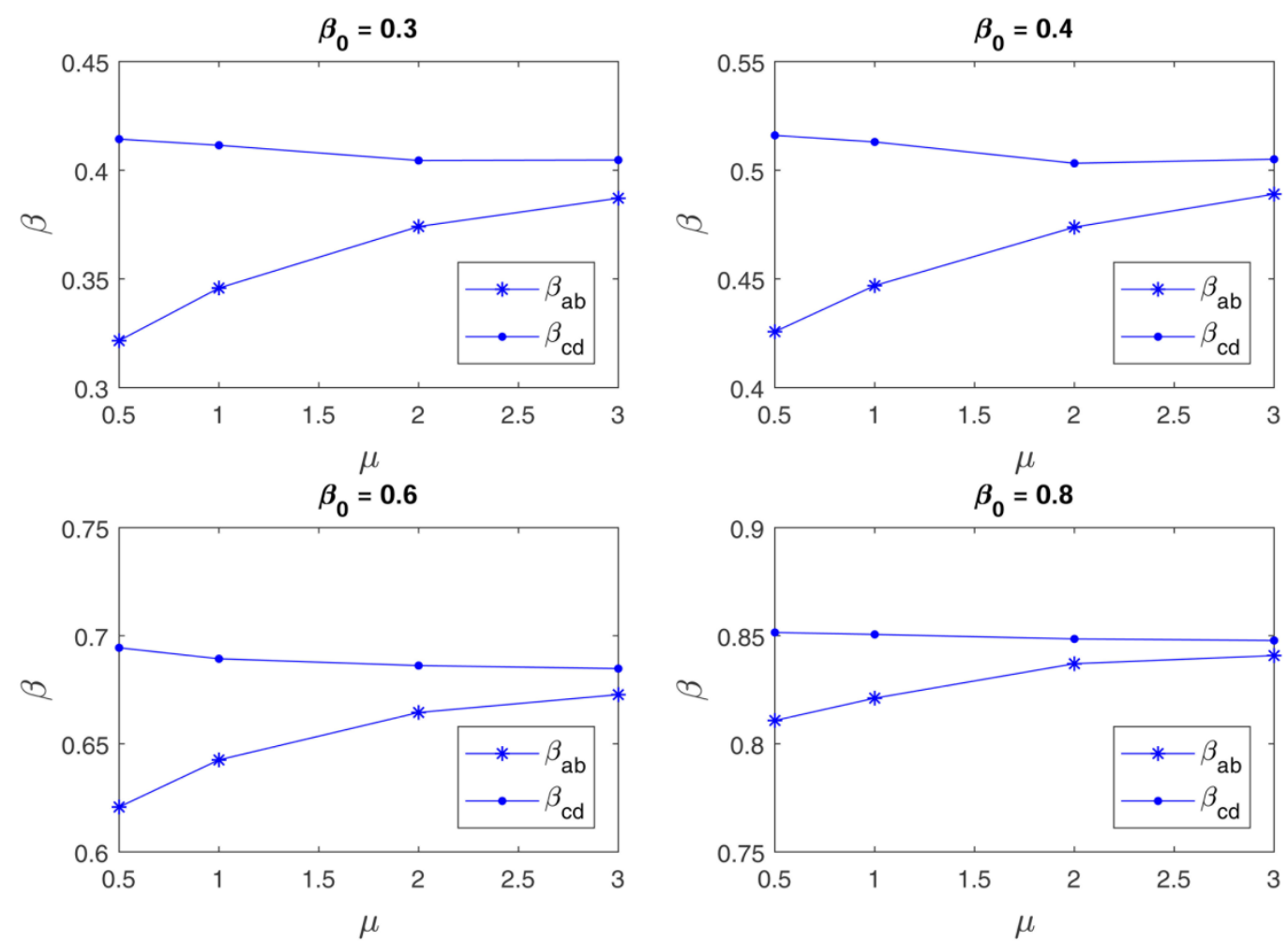

Fig. 4: Numerical prediction of the ellipticity ratio for both contact and adhesive ellipses at the pull-off moment for various $\beta_{0}$ values. 
Fig. 5 shows the adhesive regions for various elliptical contacts between two cylindrical bodies from the same material, obtained from the numerical simulations in Section 2.2.1. Following Eq. (11), the geometry of the adhesive region is summarized in a non-dimensional form. Results show that the differences between the semi-major and semi-minor axes for both contact and adhesive ellipses become smaller as $\beta_{0}$ increases.
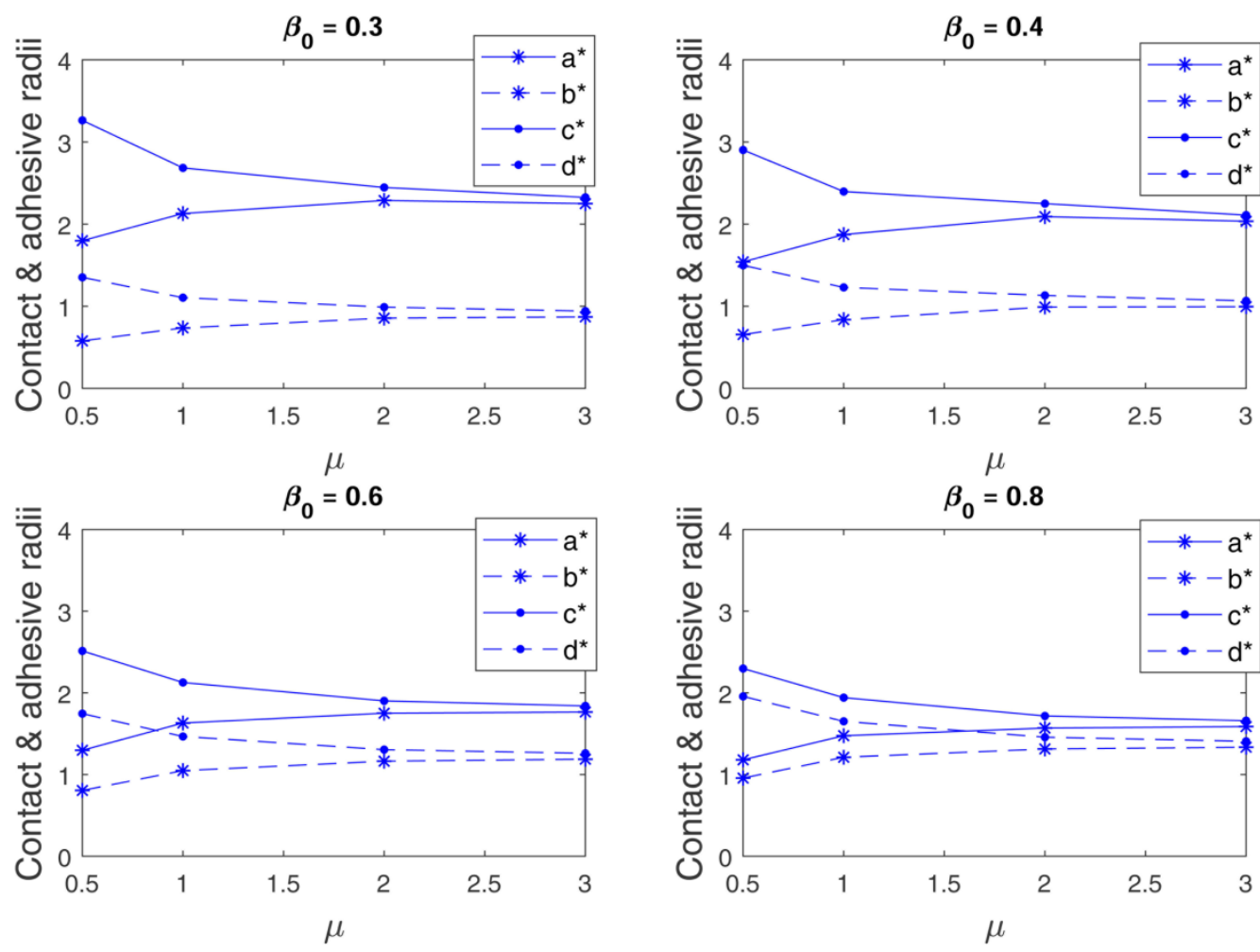

Fig. 5: Non-dimensional contact and adhesive radii of the adhesive region at the pull-off moment for various $\beta_{0}$ values.

Numerical data at the pull-off moment is then curve-fitted to obtain ellipticity ratio equations of the adhesive region boundaries, and also the semi-major axis of the contact ellipse, for various elliptical contacts.

\subsubsection{Fitting procedure}

The procedure for curve-fitting begins by using the numerical model discussed in Section 2.2.1 to calculate $\beta_{a b}$ and $\beta_{c d}$ as a function of $\theta_{\text {skew }}$ and $\mu$. The resulting numerical data is then analysed to determine the best curve-fit for independent parameters individually. All the curve-fits are obtained using the built-in fminsearch function in MATLAB.

The curve-fitting procedure for $a$ is similar to $\beta_{a b}$ and $\beta_{c d}$. However, unlike $\beta_{a b}$ and $\beta_{c d}$, $a$ has a dimensional unit; hence it is curve-fitted first into non-dimensional form $a^{*}$, as a 
function of non-dimensional parameters of $\beta_{a b}$ and $\mu$. The fitted $a^{*}$ equation is then transformed into the dimensional form a that is based on the JKR semi-major axis solution in [11], with the inclusion of the dimensional parameters of $R, \Delta Y$ and $E^{*}$ in the equation.

\section{Results}

From the curve-fitting in section 2.2.2, we have obtained the equations that can predict the values of $\beta_{a b}$ and $\beta_{c d}$ at the pull-off moment, as a function of $\mu$ and $\theta_{\text {skew. These ellipticity }}$ ratio equations at the pull-off moment are given as:

$$
\begin{aligned}
& \beta_{a b}=0.3899 \theta_{\text {skew }}{ }^{1.7669}+0.0829 \mu^{0.3316}+0.0233 \\
& \beta_{c d}=0.4139 \theta_{\text {skew }}{ }^{1.5837}+0.7199 \mu^{-0.0074}-0.5783
\end{aligned}
$$

Using $\beta_{a b}$ from Eq. (22a), the equation to predict the semi-major axis of the contact ellipse at the pull-off moment, $a$ is given as:

$$
a=\left(\frac{R^{2} \Delta \gamma}{E^{*}}\right)^{1 / 3}\left(\sinh \left(1.0499 \beta_{a b}{ }^{-0.1553}-0.0648 \mu^{-1.1945}\right)\right)^{2}
$$

When compared with the numerical model, the maximum errors of Eq. (22) are $2.67 \%$ for $\beta_{a b}$ at $\beta_{0}=0.3$, and $0.76 \%$ for $\beta_{c d}$ at $\beta_{0}=0.4$, while the minimum errors are calculated as $0.124 \%$ at $\beta_{0}=0.8$ and $0.007 \%$ at $\beta_{0}=0.3$, for $\beta_{a b}$ and $\beta_{c d}$, respectively. As for the errors of $a$ in Eq. (23), the maximum error is $2.87 \%$ at $\beta_{0}=0.4$, while the minimum error is $0.15 \%$ at $\beta_{0}=0.3$.

Fig. 6 and Fig. 7 show the effect of the Tabor parameter, $\mu$ on both $\beta_{a b}$ and $\beta_{c d}$ at the pull-off moment, with the values obtained from Eq. (22) are compared to the numerical model. The ellipticity ratios from the JKR elliptical model at the pull-off moment are also included for reference, but only in Fig. 6. The adhesive stresses in the JKR model occur only within the contact area, thus only $\beta_{a b}$ values of the JKR model are available for comparison. It is shown in Fig. 6 that $\beta_{a b}$ increases at a faster rate for lower $\mu$ values and eventually slows down as the curve approaches $\mu=3$. The result from the curve-fitted Eq. (22) is shown to approach the JKR result as $\mu$ and $\beta_{0}$ values increase. Meanwhile, in Fig. 7 , the decreasing rate for $\beta_{c d}$ is fairly constant from $\mu=0.5$ to $\mu=3$. 

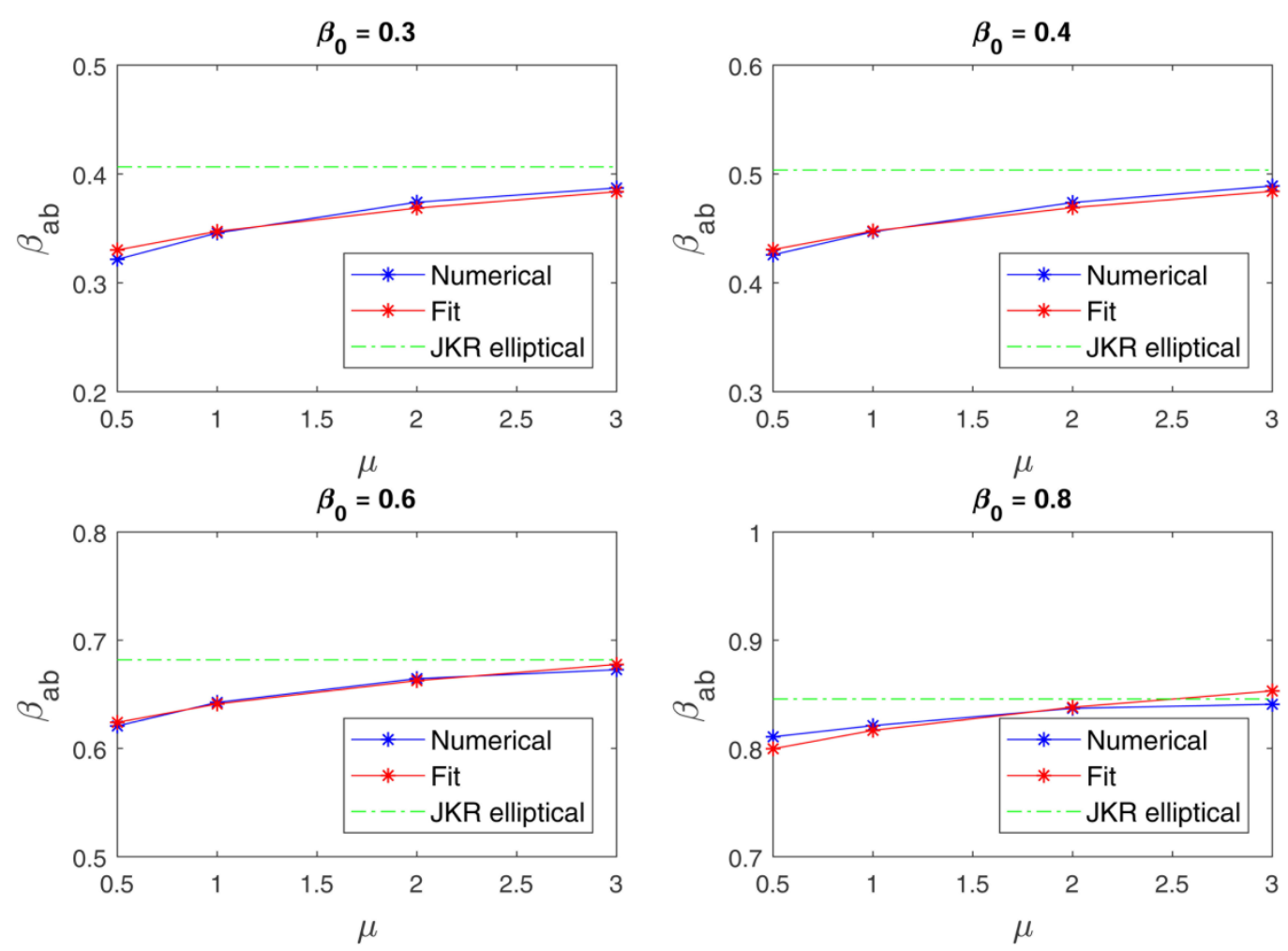

Fig. 6: Ellipticity ratio of the contact ellipse at the pull-off moment for the numerical model, the curve-fitted equation and the JKR elliptical model for various $\beta_{0}$ values.
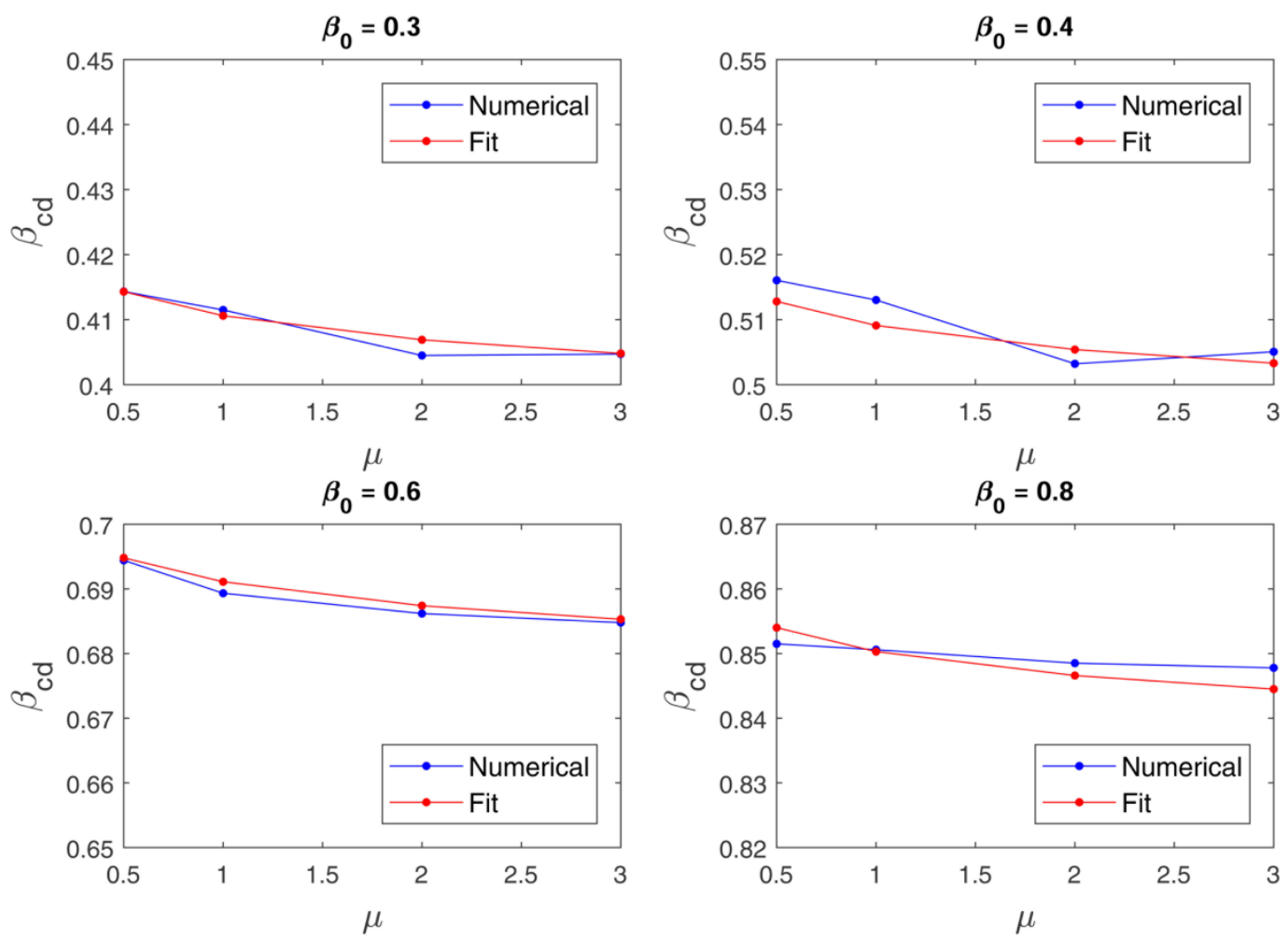

Fig. 7: Ellipticity ratio of the adhesive ellipse at the pull-off moment for the numerical model and the curve-fitted equation for various $\beta_{0}$ values. 
The non-dimensional values of the semi-major axis of the contact ellipse at the pulloff moment, $a^{*}$ from Eq. (23) and the resulting semi-major axis of the adhesive ellipse at the pull-off moment, $c^{*}$ are plotted in Fig. 8, with the numerical results. Values from Eq. (22) and Eq. (23) are used in the extended $\mathrm{DH}$ model to calculate $c^{*}$ values. The $a^{*}$ values from both numerical results and Eq. (23) are similar for all $\beta_{0}$ values, while the $c^{*}$ values obtained from the solution of Eq. (22) and Eq. (23) are higher than the numerical results.
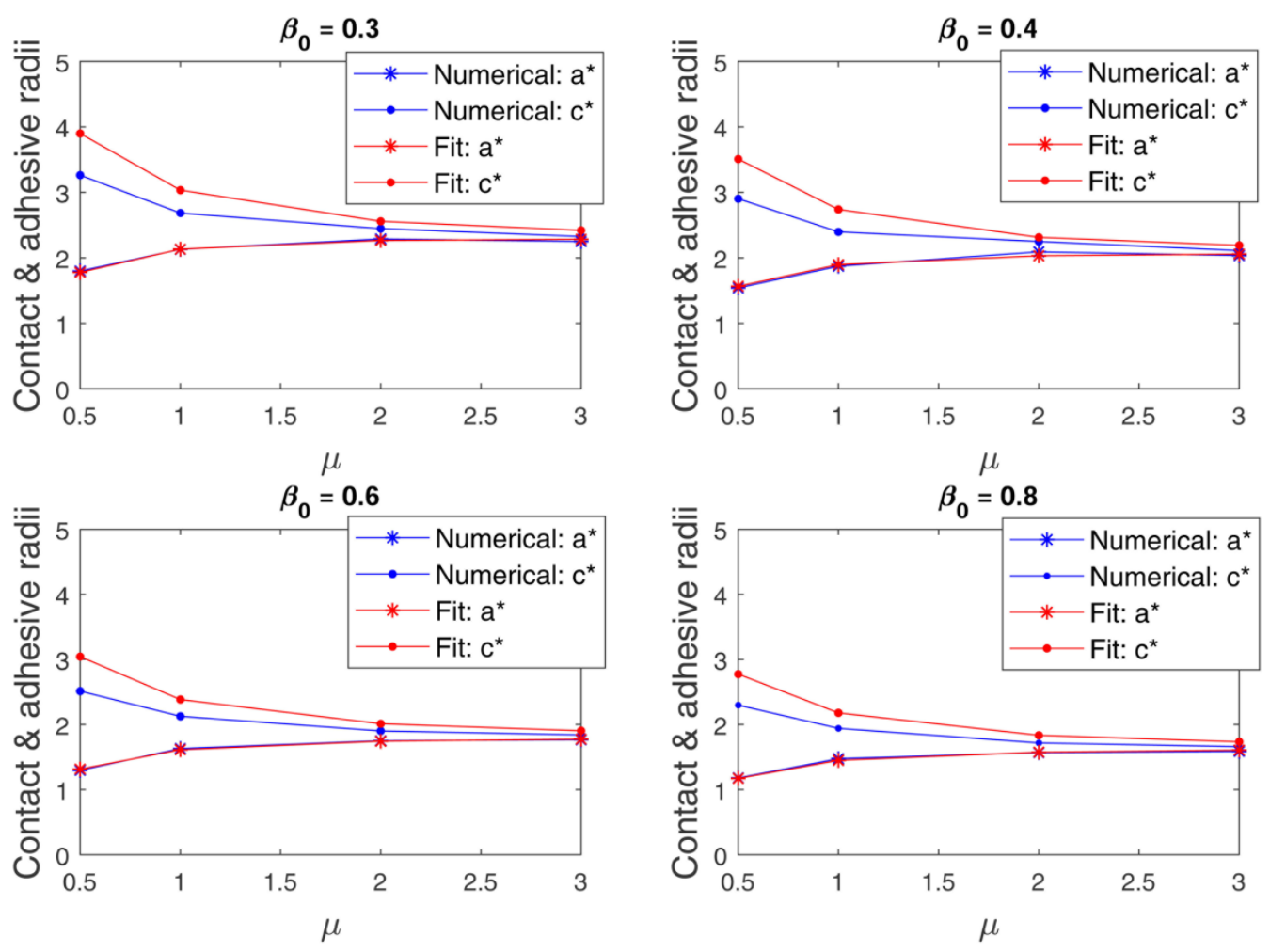

Fig. 8: Non-dimensional semi-major axis of both contact and adhesive ellipses at the pulloff moment for the numerical model and the curve-fitted equations for various $\beta_{0}$ values.

Fig. 9 shows the comparison between the pull-off force results of the numerical model, the extended DH model, the PDMS experimental results in [12] which are obtained using a graph digitizer software, and the JKR elliptical model [11]. Eq. (22) and Eq. (23) are incorporated in the extended $\mathrm{DH}$ model to predict $c^{*}$ and the scaling factor $v$ at the pulloff moment, which are then used to calculate the pull-off forces. For $\beta_{0}$ values of 0.3 and 0.4 , the curves for both numerical and extended $\mathrm{DH}$ model are similar for low $\mu$ up to $\mu=1$, where the numerical pull-off forces keep on decreasing while the pull-off force values of the extended $\mathrm{DH}$ model begin to increase. For $\beta_{0}$ value of 0.8 , the extended $\mathrm{DH}$ model predicts higher pull-off forces near the DMT domain, though before $\mu=2$ the pull-off forces of the numerical model start to exceed the extended $\mathrm{DH}$ results. 

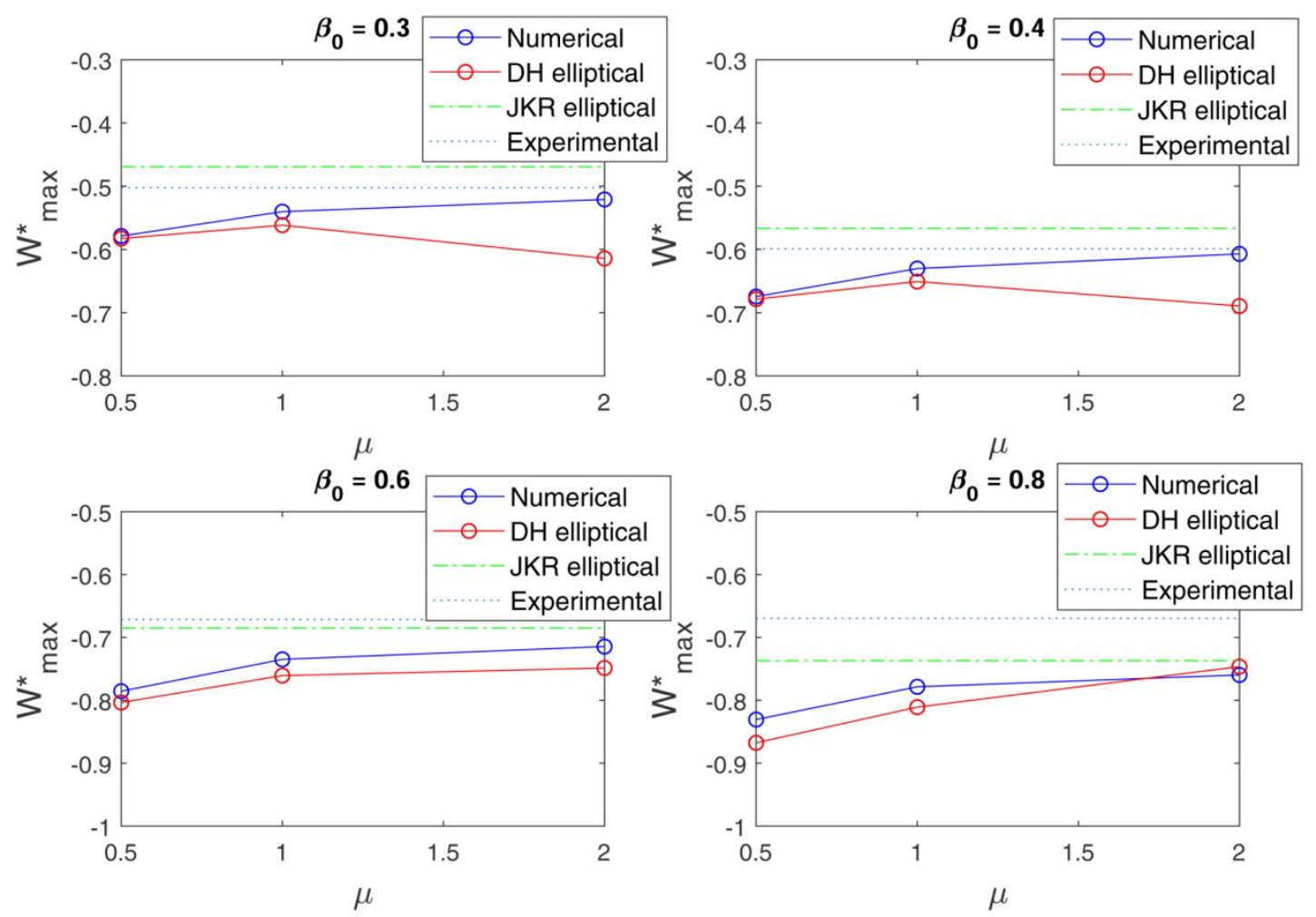

Fig. 9: Pull-off force prediction between the numerical model, the extended DH model, the JKR elliptical model and the existing experimental results for various $\beta_{0}$ values.

Some of the results in Fig. 9 are replotted in Fig. 10 with the pull-off force as a function of $\theta_{\text {skew. }}$. The curves for $\mu$ values of 0.5 and 1 are shown to have similar behavior as the JKR elliptical model. The extended $\mathrm{DH}$ model can be considered to already achieve its limit somewhere in the range of $1<\mu<2$, though this might need further research. 


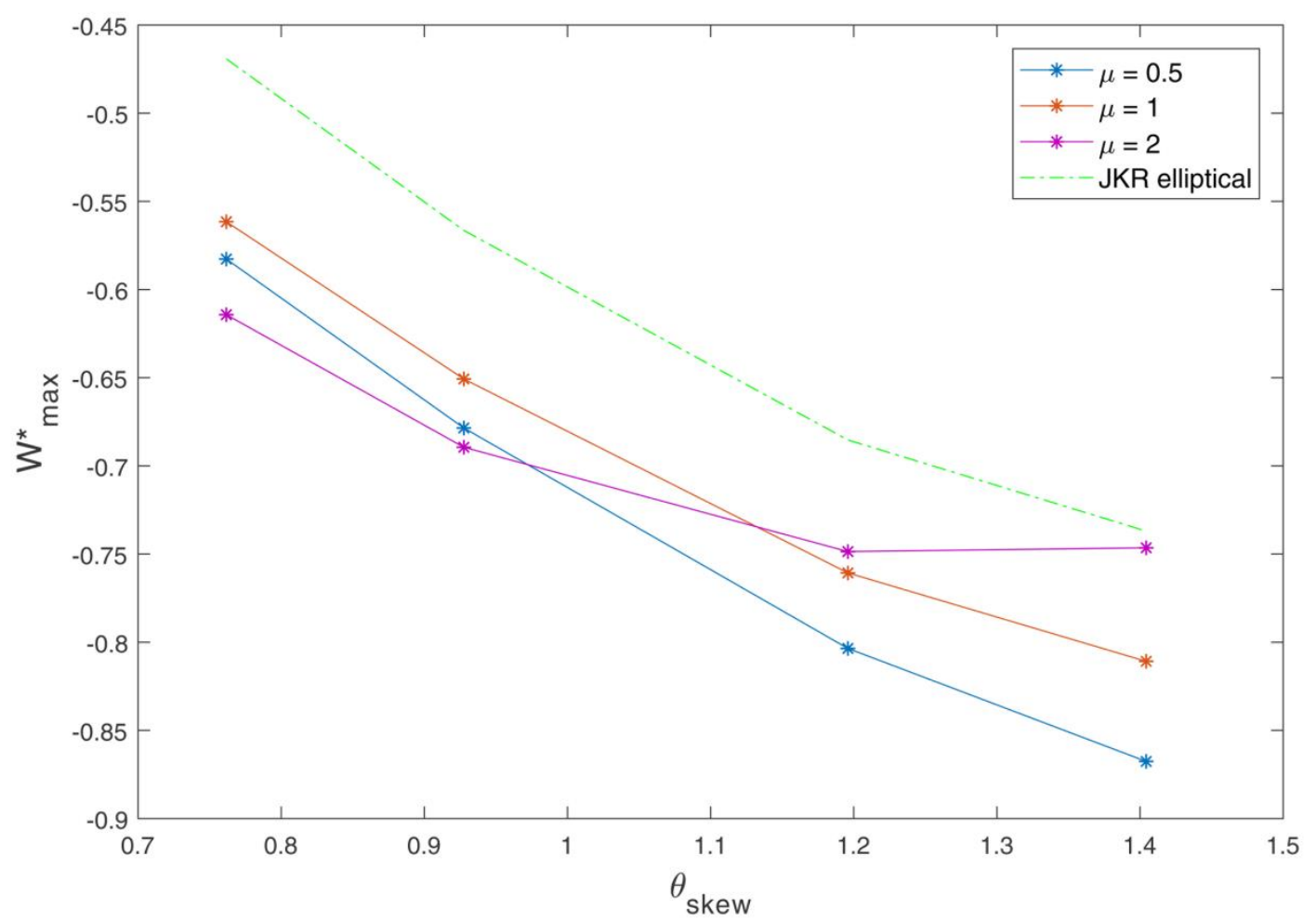

Fig. 10: Pull-off forces as a function of $\theta_{\text {skew }}$ for $\mu$ values of $0.5,1$ and 2, compared to the JKR elliptical model.

\section{Discussion}

For the curve-fitting of the ellipticity ratio data at the pull-off moment, there are many mathematical functions that can be considered to represent the data. However, by referring to the pull-off force behavior of an adhesive circular contact as discussed in [6], it is clear how the contact should behave. It should be noted that for adhesive elliptical contacts, there are no theoretical limits of the contact models in terms of rigid and highly elastic materials, unlike the adhesive circular contacts. However, in this paper, the fitting equations are developed for $0.5 \leq \mu \leq 3$, as the lower limit for the extended $\mathrm{DH}$ model is $\mu$ $=0.5$ and the upper limit for the numerical model is $\mu=3$. The values of $\beta_{a b}$ increase rapidly at low $\mu$ values that are close to the DMT domain. Materials that are considered closed to the DMT domain barely deform along the semi-major axis direction, as shown by the close value of $\beta_{a b}$ to $\beta_{0}$. The rapid increase of $\beta_{a b}$ eventually slows down as the curve is within the intermediate domain and becomes nearly stable as it approaches the JKR domain. Closed to the JKR domain, the highly elastic materials are easily deformed, resulting in high $\beta_{a b}$ values. This behavior is represented well by Eq. (22a). As for the behavior of $\beta_{c d}$, there is no reference available on how the adhesive ellipse might behave. 
Solely based on the trend of the numerical data, it is shown that the values of $\beta_{c d}$ decrease at a nearly constant manner, indicating that the effect of $\mu$ on $\beta_{c d}$ is not dominant.

Although the curve-fitting of $a$ is based on the JKR semi-major axis equation in [11], the final form of $a$ is not similar to the JKR solution. Unlike the JKR elliptical model, whose application is specific to highly elastic materials, $\mu$ does affect the pull-off force values in the extended DH model. Hence, $\mu$ is included in the fitted a equation. Even though $a^{*}$ predictions are similar for the numerical model and the extended $\mathrm{DH}$ model, the $c^{*}$ values are shown to be higher than the numerical results. As the numerical simulation is based on the MD model, it is expected to have similar work of adhesion (surface energy) as the extended DH model. Hence, the area under the curve for both models should be equal, as illustrated in Fig. 11. The higher $c^{*}$ prediction is to compensate the decreasing values of adhesive stresses, as opposed to constant adhesive stresses by the MD model in the adhesive region. For lower $\mu$, the pull-off forces predicted by both the extended $\mathrm{DH}$ model and the numerical model are close even though the fitted $c^{*}$ is larger than the $c^{*}$ predicted numerically. The difference between both $c^{*}$ values have little influence on the pull-off force prediction as both models have close results. However, the numerical errors become significant at higher $\mu$ values that are close to or already in the JKR domain, especially for low $\beta_{0}$ values due to a really small adhesive zone. At really high $\mu$, the adhesive zone is so small that the extended $\mathrm{DH}$ model cannot model such behaviour accurately. Hence, the pull-off forces predicted by the extended $\mathrm{DH}$ model have large errors compared to the numerical results.

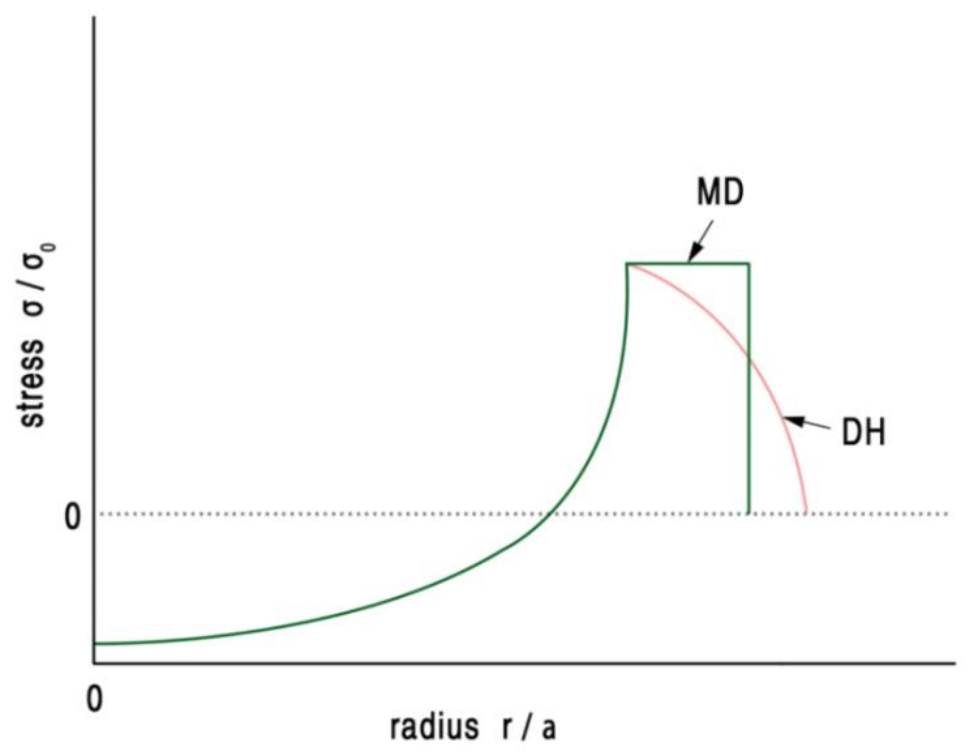

Fig. 11: Graphical representation of the adhesive stresses distribution by both $\mathrm{DH}$ and MD models. 
From Fig. 10, it is shown that the behavior of the curves for $\mu$ values of 0.5 and 1 are similar to the JKR results; the curve of $\mu=1$ follows really closely the behavior of the JKR results compared to the curve of $\mu=0.5$, signifying that it is really close to the JKR domain. However, for the curve of $\mu=2$, its behavior is totally different compared to the JKR result. This can be an indicator that at $\mu=2$, the contact is already within the JKR domain. This situation is further proved by the ratio of $c_{\text {num }}{ }^{*}$ to $a_{\text {num }}{ }^{*}$ in Table 2 , calculated from the numerical results. At $\mu=2$, the values of $c_{\text {num }}{ }^{*} / a_{\text {num }}{ }^{*}$ are really close to 1 , especially for lower $\beta_{0}$ values, a sign that the adhesive region outside the contact is really small, which is the characteristic of the JKR model. To conclude, the JKR domain is larger for elliptical contacts compared to the circular contacts, as seen by the JKR domain starts at much lower $\mu$ value than 5 , which is the application limit for the JKR model of circular contacts.

Table 2: Ratio of $c_{\text {num }}{ }^{*}$ to $a_{\text {num }}{ }^{*}$ for various contact conditions.

\begin{tabular}{|l|l|l|l|l|}
\hline \multirow{2}{*}{$\mu$} & \multicolumn{3}{|l|}{$C_{\text {num }} / a_{\text {num }}$} \\
\cline { 2 - 5 } & $\beta_{0}=0.3$ & $\beta_{0}=0.4$ & $\beta_{0}=0.6$ & $\beta_{0}=0.8$ \\
\hline 0.5 & 1.8146 & 1.8837 & 1.9379 & 1.9477 \\
\hline 1 & 1.2606 & 1.2793 & 1.3039 & 1.3157 \\
\hline 2 & 1.0692 & 1.0757 & 1.0863 & 1.0947 \\
\hline 3 & 1.0340 & 1.0371 & 1.0425 & 1.0459 \\
\hline
\end{tabular}

Unlike the JKR domain, validation for $\beta_{a b}$ values with the DMT elliptical model near the rigid domain is not possible due to the Bradley's approach for the DMT elliptical model [15]. From the pull-off force comparison for the adhesive models of circular contacts in [6], it is shown that at $\mu=0.5$, the deviation of the pull-off force prediction by the Bradley model from the $\mathrm{DH}$ model is already significant with a large error of $15 \%$. Basically, the pull-off behavior of the Bradley model is already different compared to the $\mathrm{DH}$ model at $\mu=0.5$. On the other hand, at $\mu=3$, both the DH model and the JKR model have nearly similar results with a low error of $2 \%$, even though the application of the JKR model only begins at $\mu=5$. Based from these error values, larger discrepancies between these models should be expected for elliptical contacts. Hence, for Tabor range of $0.5 \leq \mu \leq 3$, the contact model comparison is only conducted between the $\mathrm{DH}$ model and the JKR model. 


\section{Conclusions}

The work on the extended $\mathrm{DH}$ model has been continued to include the assumption of an adhesive region with boundaries that vary with load, for an accurate pull-off force prediction. The geometry of the annular adhesive region at the pull-off moment is obtained from a Dugdale-based numerical model. These numerically-obtained values are curvefitted to find the equations to predict the ellipticity ratios for both ellipses that bounded the adhesive region at the pull-off moment and the semi-major axis of the contact ellipse, and then incorporated into the extended $\mathrm{DH}$ model. The incorporated equations of the ellipticity ratio and the contact semi-major axis at the pull-off moment allow the extended $\mathrm{DH}$ model to accurately predict the pull-off force in its working domain of $0.5 \leq \mu<2$, for various elliptical contacts. It is shown that the validity domain for the JKR model is expanding and dominating adhesive elliptical contacts.

\section{Acknowledgements}

The authors gratefully acknowledge support from the Ministry of Education Malaysia, Universiti Teknikal Malaysia Melaka and Green Tribology and Engine Performance (GTriboE) research group.

\section{Appendix A: Coordinate system for governing equations}

The transformation from Cartesian to polar coordinate system for the contact and adhesive ellipses are obtained from [17], which $r_{a b}$ and $r_{c d}$ from Fig. 12 are given as:

$$
\begin{aligned}
& r_{a b}=a b / \sqrt{\left(b \cos \theta_{p}\right)^{2}+\left(a \sin \theta_{p}\right)^{2}} \\
& r_{c d}=c d / \sqrt{\left(d \cos \theta_{p}\right)^{2}+\left(c \sin \theta_{p}\right)^{2}}
\end{aligned}
$$

where

$$
\begin{aligned}
& x=r \cos \theta_{p} \\
& y=r \sin \theta_{p}
\end{aligned}
$$




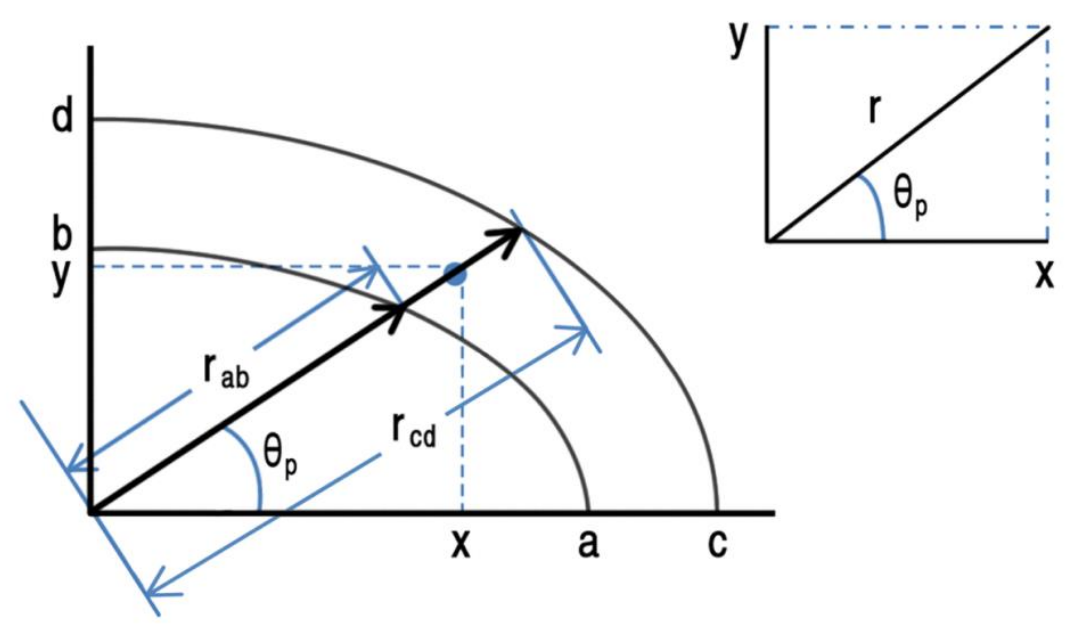

Fig. 12: Cartesian to polar coordinate system transformation for the contact and adhesive ellipses [17].

\section{Appendix B: Solutions for incomplete elliptic integrals}

$$
\begin{gathered}
L_{i n_{a b}}=\delta=\pi P_{o_{a b}} b \boldsymbol{K}\left(k_{a b}\right) \\
M_{i n_{a b}}=\pi P_{o_{a b}} \beta_{a b}\left[\boldsymbol{K}\left(k_{a b}\right)-E\left(k_{a b}\right)\right] / k_{a b}{ }^{2} a \\
N_{i n_{a b}}=\pi P_{o_{a b}} \beta_{a b}\left[\boldsymbol{E}\left(k_{a b}\right) / \beta_{a b}{ }^{2}-\boldsymbol{K}\left(k_{a b}\right)\right] / k_{a b}{ }^{2} a \\
L_{o u t_{a b}}=\pi P_{o_{a b}} b \boldsymbol{F}\left(\varphi, k_{a b}\right) \\
M_{o u t_{a b}}=\pi P_{o_{a b}} \beta_{a b}\left[\boldsymbol{F}\left(\varphi, k_{a b}\right)-\boldsymbol{E}\left(\varphi, k_{a b}\right)\right] / k_{a b}{ }^{2} a \\
N_{o u t_{a b}}=\pi P_{o_{a b}} \beta_{a b}\left[\boldsymbol{E}\left(\varphi, k_{a b}\right)-{k_{a b}}^{2} \boldsymbol{F}\left(\varphi, k_{a b}\right)-k_{a b}{ }^{2} \sin \varphi \cos \varphi / \sqrt{1-\left(k_{a b} \sin \varphi\right)^{2}}\right] / \\
k_{a b}{ }^{2} k_{a b}{ }^{2} a \\
\quad(\mathrm{~B}-6) \\
L_{i n_{c d}}=\pi P_{o_{c d}} d \boldsymbol{K}\left(k_{c d}\right) \\
M_{i n_{c d}}=\pi P_{o_{c d}} \beta_{c d}\left[\boldsymbol{K}\left(k_{c d}\right)-E\left(k_{c d}\right)\right] / k_{c d}{ }^{2} c \\
N_{i n_{c d}}=\pi P_{o_{c d}} \beta_{c d}\left[\boldsymbol{E}\left(k_{c d}\right) / \beta_{c d}{ }^{2}-\boldsymbol{K}\left(k_{c d}\right)\right] / k_{c d}{ }^{2} c
\end{gathered}
$$




\section{References}

1. Kendall, K.: Adhesion: Molecules and mechanics. Science 263(5154), 1720-1725 (1994)

2. Derjaguin, B.V., Muller, V.M., Toporov, Y.P.: Effect of contact deformations on the adhesion of particles. J. Colloid Interface Sci. 53,314-325 (1975)

3. Muller, V.M., Derjaguin, B.V., Toporov, Y.P.: On two methods of calculation of the force of sticking of an elastic sphere to a rigid plane. Colloids Surf. 7, 251-259 (1983)

4. Johnson, K.L., Kendall, K., Roberts, A.D.: Surface energy and the contact of elastic solids. Proc. R. Soc. Lond. Ser. A 324, 301-313 (1971)

5. Maugis, D.: Adhesion of spheres: The JKR-DMT transition using a Dugdale model. J. Colloid Interface Sci. 150(1), 243-269 (1992)

6. Greenwood, J.A., Johnson, K.L.: An alternative to the Maugis model of adhesion between elastic spheres. J. Phys. D Appl. Phys. 31(22), 3279-3290 (1998)

7. Johnson, K.L., Greenwood, J.A.: An adhesion map for the contact of elastic spheres. J Colloid Interface Sci. 192(2), 326-333 (1997)

8. Tabor, D.: Surface forces and surface interactions. J. Colloid Interface Sci. 58(1), 2$13(1977)$

9. Yin Zhang (2012) Transitions between different contact models, journal of adhesion science and Technology, 22:7, 699-715, DOI:10.1163/156856108X309648

10. Johnson, K.L.: Contact Mechanics. Cambridge University Press, Cambridge (1985)

11.Johnson, K.L., Greenwood, J.A.: An approximate JKR theory for elliptical contacts. J. Phys. D Appl. Phys. 38(7), 1042-1046 (2005)

12.Sümer, B., Onal, C., Aksak, B., Sitti, M.: An experimental analysis of elliptical adhesive contact. J. Appl. Phys. 107(11), 113512 (2010)

13. Jin, C., Jagota, A., Hui, C.: An easy-to-implement numerical simulation method for adhesive contact problems involving asymmetric adhesive contact. J. Phys. D Appl. Phys. 44(40), 405303 (2011)

14. Ciavarella, M.: An approximate JKR solution for a general contact, including rough contacts. J. Mech. Phys. Solids. 114, 209-218 (2018)

15. Wu, X., Dzenis. Y.A.: Adhesive contact in filaments. J. Phys. D Appl. Phys. 40, 4276-4280 (2007)

16. Bradley, R.S.: The cohesive force between solid surfaces and the surface energy of solids. Philos. Mag. 13, 853-862 (1932) 
17.Zini, N.H.M., de Rooij, M.B., Bazrafshan, M., Ismail, N., Schipper, D.J.: Extending the double-Hertz model to allow modeling of an adhesive elliptical contact. Tribol. Let. 66: 30 (2018)

18.Zini, N.H.M., de Rooij, M.B., Fadafan, M.B.A. et al. Tribol Lett (2018) 66:99. https://doi.org/10.1007/s11249-018-1050-x

19. Bazrafshan, M., de Rooij, M.B., Valefi, M., Schipper, D.J.: Numerical method for the adhesive normal contact analysis based on a Dugdale approximation. Tribol. Int. 112, 117-128 (2017)

20.Popov,V.L., Pohrt, R. \& Li, Q. Friction (2017) 5: 308. https://doi.org/10.1007/s40544-017-0177-3 


\title{
Paper D
}

\section{Adhesive nanofiber contacts in electrospun nanoyarns}

\author{
N.H.M. Zini ${ }^{1,2,}$, M.B. de Rooij ${ }^{1}$, D.J. Schipper ${ }^{1}$ \\ 1) Department of Surface Technology and Tribology, Faculty of Engineering Technology, \\ University of Twente, the Netherlands \\ 2) Faculty of Mechanical Engineering, Universiti Teknikal Malaysia Melaka, \\ Hang Tuah Jaya, 76100 Durian Tunggal, Melaka, Malaysia
}

Submitted to Textile Research Journal 

Abstract In contact between single fibers, the presence of adhesion is acknowledged and has been deeply researched. However, a general understanding is still lacking in terms of adhesive contacts between fibers in hierarchical structures. Hierarchical fiber structures like nanoyarns acknowledge that adhesion is present in the contacts between nanofibers, though the general understanding of the mechanism is still lacking. This work sets out to investigate the significance of the presence of adhesion in the contact between nanofibers in a nanoyarn. The effect of the electrospinning process parameters on adhesion between nanofibers in a nanoyarn is also determined. Contact and load properties for the nanofiber contacts in electrospun nanoyarns are obtained from a geometrical model of a low-order hierarchical structure; these parameters are then used as inputs to the adhesive contact model to predict adhesion between nanofibers in a nanoyarn. Adhesion is indeed significant between nanofiber contacts in electrospun nanoyarns and is shown to be influenced by the nanofiber radius, the number of nanofibers in a nanoyarn, the nanofiber twisting speed and the nanoyarn reeling speed; the effect of the nanofiber twisting speed on the adhesive nanofiber contacts is opposite to that of the nanoyarn reeling speed.

Keywords: Adhesion; Elliptical contact; JKR elliptical; Hierarchical structure; Nanofiber contacts

\section{List of symbols}

$\begin{array}{ll}r & \text { Nanofiber radius } \\ D & \text { SWHS structure diameter } \\ P & \text { SWHS structure pitch } \\ \alpha_{1} & \text { Helical angle of the nanoyarn } \\ \beta & \text { Twist angle of a strand } \\ R_{1} & \text { Center distribution } \\ \omega_{1} & \text { Nanofiber twisting speed } \\ \omega_{2} & \text { Nanoyarn reeling speed } \\ n & \text { Number of nanofibers in a nanoyarn } \\ a, b & \text { Semi-major and semi-minor axes of the contact ellipse at the pull-off } \\ \theta_{\text {skew }} & \text { moment } \\ E^{*} & \text { Angle between two cylindrical bodies } \\ R^{\prime}, R^{\prime \prime} & \text { Reduced Young's modulus } \\ R=\sqrt{R^{\prime} R^{\prime \prime}} & \text { Equincipal relative radii of curvature }\end{array}$


$\mu \quad$ Tabor parameter

$\Delta \gamma \quad$ Work of adhesion

$W_{f} \quad$ Normal contact force between two nanofibers

$W_{y} \quad$ Axial load of the nanoyarn

$W_{J K R} \quad$ Pull-off force between adjacent nanofibers

\section{Introduction}

Fibers have long been utilized by mankind in various applications. Fibers can be divided into two types: natural and synthetic. In contrast to natural fibers that are produced from animals and plants, synthetic fibers are man-made, created in laboratories. Both natural and synthetic fibers consist of hierarchical structures, though the structures of synthetic fibers are typically simpler than those of natural hierarchical structures.

A hierarchical structure is a structure that has more than one length scale. The structural hierarchy can significantly affect the bulk material properties; one of the advantages of utilizing hierarchical structures is that the structures typically have high strength-to-weight ratios [1]. Various levels of hierarchical fiber structures can be explained as follows: the basic component is the fibers, which form the basic structure - yarns; these structures are obtained by braiding or twisting a group of fibers together. There are also yarns that are made from parallel fibers with no twisting involved. For complex structures such as ropes, the next higher levels can consist of rope yarns, strands, core, subropes and cover [2], as shown in Figure 1.

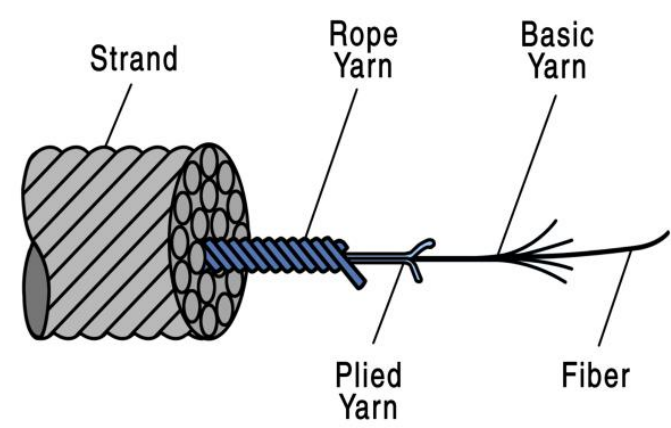

Fig. 1: Example of a hierarchical structure of ropes. 
There are also simple, low-order hierarchical structures that consist of fewer levels, such as electrospun nanoyarns. Electrospinning is a straightforward nanoyarn production system that uses an electric force to draw charged threads of polymer solutions with fiber diameters in the order of nanometers [3]. A polymer solution is forced through a syringe; a solution drop is then formed at the needle tip. A high voltage is applied to the needle, which induces electric charges within the fluid. When the applied voltage is greater than the critical voltage, the repulsive force within the charged solution is greater than its surface tension and a jet erupts from the tip of the needle. As this jet travels through the air, the solvent evaporates, and the polymer fiber is gathered by a funnel-shaped collector, which functions as a negative electrode [4,5]. The collector rotates and exerts a twist on the nanofiber bundles to form a nanoyarn; the nanoyarn is then wound on a yarn rolling device or wrapped around another yarn as protection. The electrospinning process is simplified graphically in Fig. 2(a). Nanofibers have diameters in the nanometer range, and a ratio of length to diameter of 100:1 [3]. Hence, during the electrospinning process nanofibers are prone to adhesion-induced deformations.

Adhesion is caused by intermolecular forces between two surfaces and becomes increasingly significant at the fiber level due to a high surface-to-volume ratio [6]. Adhesion causes two surfaces to remain in contact even though the applied load is removed; a 'negative' force termed as the pull-off force is required to separate the surfaces at a certain pull-off moment. Adhesion influences the fiber-on-fiber contact by increasing the contact area, consequently increasing friction between fibers and affecting phenomena like bundle spreading. There are four main adhesive models that can be used to describe the adhesive contact between fibers: (1) the Johnson-Kendall-Roberts (JKR) model [7], (2) the Derjaguin-Muller-Toporov (DMT) model [8], (3) the Maugis-Dugdale (MD) model [9] and (4) the Double-Hertz (DH) model [10]. The JKR model in [7] is applicable for highly elastic materials while the DMT model is applicable to relatively rigid materials [8]. Both the MD [9] and the $\mathrm{DH}$ [10] models are suitable for materials that are neither rigid nor highly elastic. All these four models were initially developed for circular contacts and have been extended to describe the line contacts: [11] for the JKR-based adhesion, [12, 13] for the MD-based adhesion and [14] for the DH-based adhesion. For elliptical contacts, currently there are three adhesive models available: (1) the DMT based model with Bradley's approach for the adhesive stress [15], (2) the approximate JKR model [16] and (3) the extended $\mathrm{DH}$ model [17]. Incorporated with the geometrical solutions on the adhesive region obtained numerically from [18], the extended $\mathrm{DH}$ model is shown to be suitable for elliptical contacts with various conditions. However, it is also shown that for elliptical 
contacts, the DH model transitions to the JKR model at a much lower Tabor parameter $\mu$. Investigations on adhesive contacts in hierarchical structures have been widely conducted for biological structures especially gecko [19-21]; however, no research has been done on adhesive fiber contacts in a hierarchical structure. Studies on adhesive fiber contacts are normally done for contacts between single fibers, as seen in [22-24] for nanofibers. For a low-order hierarchical structure like electrospun nanoyarns that consist of one component and one structure, adhesion may be present in the contact between nanofibers in the nanoyarn. There is no research available to verify the significance of the presence of adhesion in the nanofiber contacts in electrospun nanoyarns.

This paper investigates the significance of the presence of adhesion in a low-order hierarchical structure of electrospun nanoyarns. The effects of the electrospinning parameters on the adhesive nanofiber contacts are also analysed.

\section{$2 \quad$ Modelling nanofiber contacts in electrospun nanoyarns}

At the present time there is no fiber-based geometry model that incorporates the hierarchical structure of an electrospun nanoyarn. As the purpose of this study is to obtain the contact mechanics of adhesive nanofiber contacts in electrospun nanoyarns, other applications with similar hierarchical structure are utilized. 


\subsection{Stranded Wire Helical Spring model}

The nanofiber electrospinning process is similar to the manufacturing process of a Stranded Wire Helical Spring (SWHS). SWHS is a unique helical spring, normally made up of several steel wires that are tied up to form a multilayer and coaxial strand, which is then twisted to form a helical spring, with the same direction as the spiral [25]. Typically, it is assumed that the wires in the cable of SWHS have frictionless contact between them. Based from the results in [26], the contacts between stranded wires inside the cable are elliptical contacts, rather than line contacts. This becomes the foundation of the SWHS model, modelling the elliptical contacts between the stranded wires in the cable. When the structure of both SWHS and electrospun nanoyarns are compared, it can be seen that the component for SWHS (stranded wires) is similar to the component of the electrospun nanoyarns (nanofibers), while the cable is similar to the nanoyarns. The translation of the nanofiber electrospinning process to the SWHS geometrical model is illustrated graphically in Fig. 2(a) and Fig. 2(b).

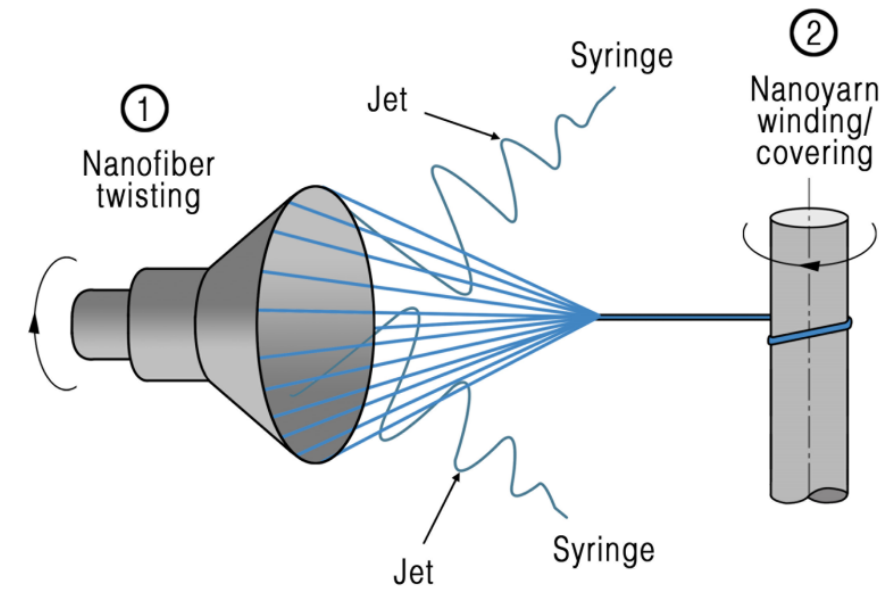

(a)

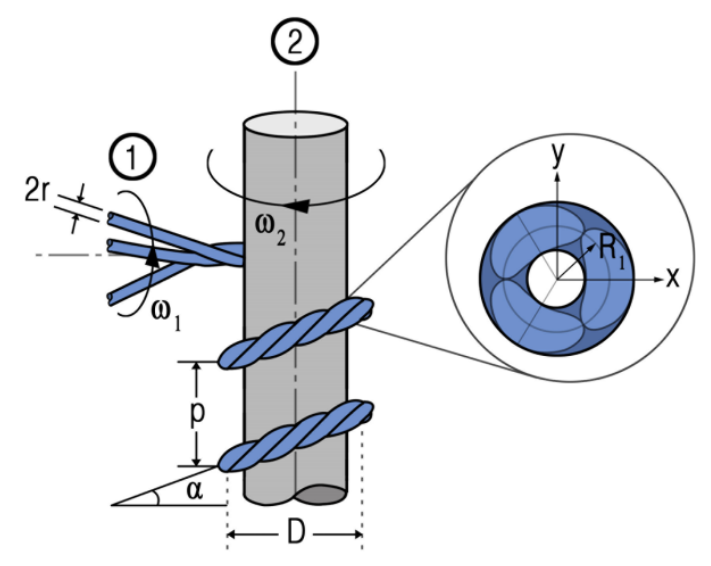

(b)

Fig. 2: (a) Nanoyarn electrospinning process (b) SWHS geometrical representation for the nanoyarn electrospinning process. 


\subsubsection{Geometrical analysis}

The skew angle between adjacent fibers in the protective yarn, $\theta_{\text {skew }}$ can be determined using a coordinate transformation, as done by [25]:

$$
\cos \theta_{\text {skew }}=\frac{\vec{a}_{1} \cdot \vec{a}_{2}}{\left|\vec{a}_{1}\right| \cdot\left|\vec{a}_{2}\right|}
$$

where the tangential vectors in any point of the central line of two adjacent fibers, $\vec{a}_{1}$ and $\vec{a}_{2}$, are given as:

$$
\begin{gathered}
\vec{a}_{1}=\left\{\begin{array}{c}
x_{1}{ }^{\prime}=-\omega_{2} D \sin \left(\omega_{2} t\right)+r\left(\sin \alpha_{1} \cdot \omega_{1}-\omega_{2}\right) \cdot \cos \left(\omega_{1} t\right) \sin \left(\omega_{2} t\right) \\
+r\left(\sin \alpha_{1} \cdot \omega_{2}-\omega_{1}\right) \cdot \sin \left(\omega_{1} t\right) \sin \left(\omega_{2} t\right) \\
y_{1}{ }^{\prime}=\omega_{2} D \cos \left(\omega_{2} t\right)+r\left(\omega_{2}-\sin \alpha_{1} \cdot \omega_{1}\right) \cdot \cos \left(\omega_{1} t\right) \cos \left(\omega_{2} t\right) \\
-r\left(\sin \alpha_{1} \cdot \omega_{2}-\omega_{1}\right) \cdot \sin \left(\omega_{1} t\right) \sin \left(\omega_{2} t\right) \\
z_{1}{ }^{\prime}=\omega_{1} r \cos \alpha_{1} \cos \left(\omega_{1} t\right)+D \omega_{2} \tan \alpha_{1}
\end{array}\right. \\
\vec{a}_{2}=\left\{\begin{array}{c}
x_{2}{ }^{\prime}=-\omega_{2} D \sin \left(\omega_{2} t\right)+r\left(\sin \left(\alpha_{1}\right) \cdot \omega_{1}-\omega_{2}\right) \cdot \cos \left(\omega_{1} t+\frac{2 \pi}{n}\right) \sin \left(\omega_{2} t\right) \\
+r\left(\sin \alpha_{1} \cdot \omega_{2}-\omega_{1}\right) \cdot \sin \left(\omega_{1} t+\frac{2 \pi}{n}\right) \sin \left(\omega_{1} t+\frac{2 \pi}{n}\right) \sin \left(\omega_{2} t\right) \\
y_{2}{ }^{\prime}=\omega_{2} D \cos \left(\omega_{2} t\right)+r\left(\omega_{2}-\sin \left(\alpha_{1}\right) \cdot \omega_{1}\right) \cdot \cos \left(\omega_{1} t+\frac{2 \pi}{n}\right) \cos \left(\omega_{2} t\right) \\
-r\left(\omega_{1}-\sin \alpha_{1} \cdot \omega_{2}\right) \cdot \sin \left(\omega_{1} t+\frac{2 \pi}{n}\right) \sin \left(\omega_{2} t\right) \\
z_{2}{ }^{\prime}=\omega_{1} r \cos \left(\alpha_{1}\right) \cos \left(\omega_{1} t+\frac{2 \pi}{n}\right)+D \omega_{2} \tan \left(\alpha_{1}\right)
\end{array}\right.
\end{gathered}
$$

$x^{\prime}, y^{\prime}$ and $z^{\prime}$ are the axis coordinates of the central line of the nanofibers, as a function of reeling time $t, n$ is the number of nanofibers in a nanoyarn, $\alpha_{1}$ is the helical angle of the nanofiber, $r$ is the nanofiber radius, $D$ is the diameter of the SWHS structure, $\omega_{1}$ is the nanofiber twisting speed and $\omega_{2}$ is the nanoyarn reeling speed. The basic geometrical equations are listed in Appendix $\mathrm{A}$.

\subsubsection{Force analysis}

Based from the analysis in [26], the general normal contact force value $W_{f}$ between any two nanofibers in a nanoyarn is given as:

$$
W_{f}=W_{y} \cos \alpha /(n-1)
$$

where $W_{y}$ is the axial load. From Fig. 2(b), the relation between the nanoyarn wrapping angle $\alpha$, the nanoyarn pitch $p$ and $D$ can be expressed as:

$$
p=\sqrt{(\pi D / \cos \alpha)^{2}-(\pi D)^{2}}
$$


Due to the limitation of existing literature, several assumptions have to be made to use the SWHS geometrical model to represent nanofiber contacts in electrospun nanoyarns:

1. $D$ from Fig. 2(b) is obtained from:

$$
D=D_{\text {core }}+d
$$

where $D_{\text {core }}$ represents the diameter of the core yarn/winding roller and $d$ is the diameter of the twisted nanoyarn.

2. The relation between nanofiber pitch $S, d$ and $n$ is given in [27] as:

$$
S / d \geq 2 n
$$

which means that the ratio of $S / d$ should be equal to or greater than twice the value of $n$. It is stated that the larger the $S / d$ ratio is, the smaller the relative errors are, for the same number of wires in the strand. In this study, the ratio of $S / d$ is set to be seven times greater than the $n$ values, hence $S$ can be expressed as:

$$
S=7 d n
$$

\subsection{Adhesive contact modelling of adjacent fibers in the protective yarn}

The JKR elliptical model in [16] is used to model adhesion in the nanofiber contacts within the hierarchical structure of electrospun nanoyarns. When two nanofibers are brought into contact, the normal load is given as:

$$
W_{J K R}=2 \pi a b\left[p_{1}-\left(\Delta_{1} a^{2}+\Delta_{2} b^{2}\right) / 3\right]
$$

The maximum negative value of $W_{J K R}$ is obtained at the pull-off moment, where the separation between the bonded nanofibers starts to occur. The semi-major axis of the contact area $a$ is given as:

$$
a^{3 / 2}=2 R \sqrt{\frac{2 \Delta \gamma}{\pi E^{*}}} \frac{(b / a)^{1 / 2}\left(1-(b / a)^{1 / 2}\right)}{\Delta_{2}^{\prime}(b / a)^{2}-\Delta_{1}{ }^{\prime}}
$$

Given is:

$$
p_{1}=\frac{\Delta_{1} a^{5 / 2}-\Delta_{2} b^{5 / 2}}{a^{1 / 2}-b^{1 / 2}}
$$

where $\Delta_{1}$ and $\Delta_{2}$ can be obtained by solving:

$$
\left[\begin{array}{c}
(\boldsymbol{D}+\boldsymbol{C}) \Delta_{1}{ }^{\prime}-\left(b^{2} / a^{2}\right) \boldsymbol{C} \Delta_{2}{ }^{\prime} \\
-\boldsymbol{C} \Delta_{1}{ }^{\prime}+\left(\boldsymbol{B}+\left(b^{2} / a^{2}\right) \boldsymbol{C}\right) \Delta_{2}{ }^{\prime}
\end{array}\right]=\left[\begin{array}{c}
\lambda \\
1 / \lambda
\end{array}\right]
$$

$\boldsymbol{B}, \boldsymbol{C}, \boldsymbol{D}$ are complete elliptic integrals, which related equations can be found in Appendix B. Next, the relation between $\lambda, R^{\prime}, R^{\prime \prime}$ and $R$ is given as:

$$
\begin{aligned}
\frac{1}{R^{\prime}} & =\frac{\lambda}{R} \\
\frac{1}{R^{\prime \prime}} & =\frac{1}{\lambda R}
\end{aligned}
$$


The geometrical parameters of the electrospun nanoyarn structure can result in the nanofiber contacts having less elastic behaviour, whereas in other conditions with similar contact properties, such as single adhesive nanofiber contacts, the interaction can be considered highly elastic. This can be analysed by using Tabor parameter $\mu$ developed by [28]. $\mu$ can be obtained by finding the ratio of neck height to the equilibrium separation, which is expressed by:

$$
\mu=\sigma_{0}\left(R / E^{* 2} \Delta \gamma\right)^{1 / 3}
$$

where $\sigma_{0}$ is the maximum adhesive stresses, $R$ is the equivalent radius, $E^{*}$ is the reduced Young's modulus and $\Delta y$ is the surface energy. "Rigid" materials will have low $\mu$ while highly elastic materials will have higher $\mu$.

\section{Results}

The presence of adhesion between nanofibers in electrospun nanoyarns is investigated on nylon 6,6 nanofibers. Table 1 lists the material properties while Table 2 lists the electrospinning properties. For the current analysis, the chosen applied load is $10 \%$ from the breaking load of nylon 6 nanofibers, obtained from [29]. Although they are different in terms of chemical structures, both nylon 6 and nylon 6,6 share the same mechanical properties. Parameters that affect adhesion in the nanofiber contacts will be determined, with the values in Table 1 and Table 2 being taken as the reference properties. A parameter study will be performed so as to analyse the significance of the presence of adhesion between nanofibers in an electrospun nanoyarn.

Table 1: Properties of the nanofiber.

\begin{tabular}{|l|c|c|c|}
\hline Properties & Nylon 6,6 & Unit & Ref. \\
\hline Transverses elastic modulus, $E_{\text {trans }}$ & 3 & GPa & {$[30]$} \\
\hline Poisson's ratio, $\vartheta$ & 0.39 & - & {$[30]$} \\
\hline Work of adhesion & 46.5 & $\mathrm{~mJ} / \mathrm{m}^{2}$ & {$[30]$} \\
\hline Interatomic spacing, $\mathrm{z}_{0}$ & 4.4 & $\AA$ & {$[30]$} \\
\hline
\end{tabular}


Table 2: Properties of the electrospinning process.

\begin{tabular}{|l|c|c|c|}
\hline Properties & Value & Unit & Ref. \\
\hline Helix angle, $\alpha$ & $\pi / 4$ & $\mathrm{rad}$ & \\
\hline Core diameter, $D_{\text {core }}$ & 2 & $\mathrm{~mm}$ & \\
\hline Applied load, $W$ & 15.2 & $\mu \mathrm{N}$ & {$[29]$} \\
\hline Nanofiber radius, $r$ & 800 & $\mathrm{~nm}$ & {$[31]$} \\
\hline Twisting speed, $\omega_{1}$ & $2 \pi / 3$ & $\mathrm{rad} / \mathrm{s}$ & {$[5]$} \\
\hline Reeling speed, $\omega_{2}$ & $\pi / 6$ & $\mathrm{rad} / \mathrm{s}$ & {$[5]$} \\
\hline
\end{tabular}

\subsection{Calculation steps}

The steps to obtain the results are listed below:

i. Set the value for $\alpha, D_{\text {core }}, r, F, \omega_{1}, \omega_{2}$ and $n$.

ii. Calculate $p$ from Eq. (4), $D$ from Eq. (5) and $S$ from Eq. (7).

iii. Calculate the basic geometrical parameters in Appendix A.

iv. Calculate $\theta_{\text {skew }}$ from Eq. (1) and Eq. (2).

v. Determine $F_{n}$ from Eq. (3) and Eq. (4).

vi. Determine $\mu$ from Eq. (13).

vii. Use $\theta_{\text {skew }}$ and $F_{n}$ as the input for the JKR elliptical model to predict pull-off force in Eq. (8) and the contact area in Eq. (9).

\subsection{The effect of the number of nanofibers in a nanoyarn and the nanofiber radius}

Fig. 3 shows the effect of nanofiber radius on the adhesive contacts, which also shows the effect of the number of nanofibers in nanoyarns. The larger nanofiber radius has no effect on the $\theta_{\text {skew }}$ values, as the values remain unchanged. However, the larger nanofiber radius is shown to result in higher $\mu$, larger contact area and higher pull-off force. For materials in the JKR domain, the resulting contact deformation is larger and the pull-off force is lower compared to the less elastic materials. Yet, for nanoyarns that consist of many highly elastic nanofibers the spaces between nanofibers are limited, hence higher pull-off force is needed to separate the nanofibers in contact. It should be noted that for nanofiber radius of $800 \mathrm{~nm}$ at $n=7$, the contact area and the pull-off force cannot be predicted as the $\theta_{\text {skew }}$ is too low for the JKR elliptical contact model. In general, a higher number of nanofibers in nanoyarns will have lower $\theta_{\text {skew, }}$ higher $\mu$ values, larger contact area and greater pull-off force.

In terms of the effect of higher applied load which is chosen at $30 \%$ of the breaking load from [29], there is no change in the adhesive contacts apart from affecting the load 
between nanofibers in contact; change in load results only in a larger Hertzian contact area, which is a characteristic of a JKR contact.
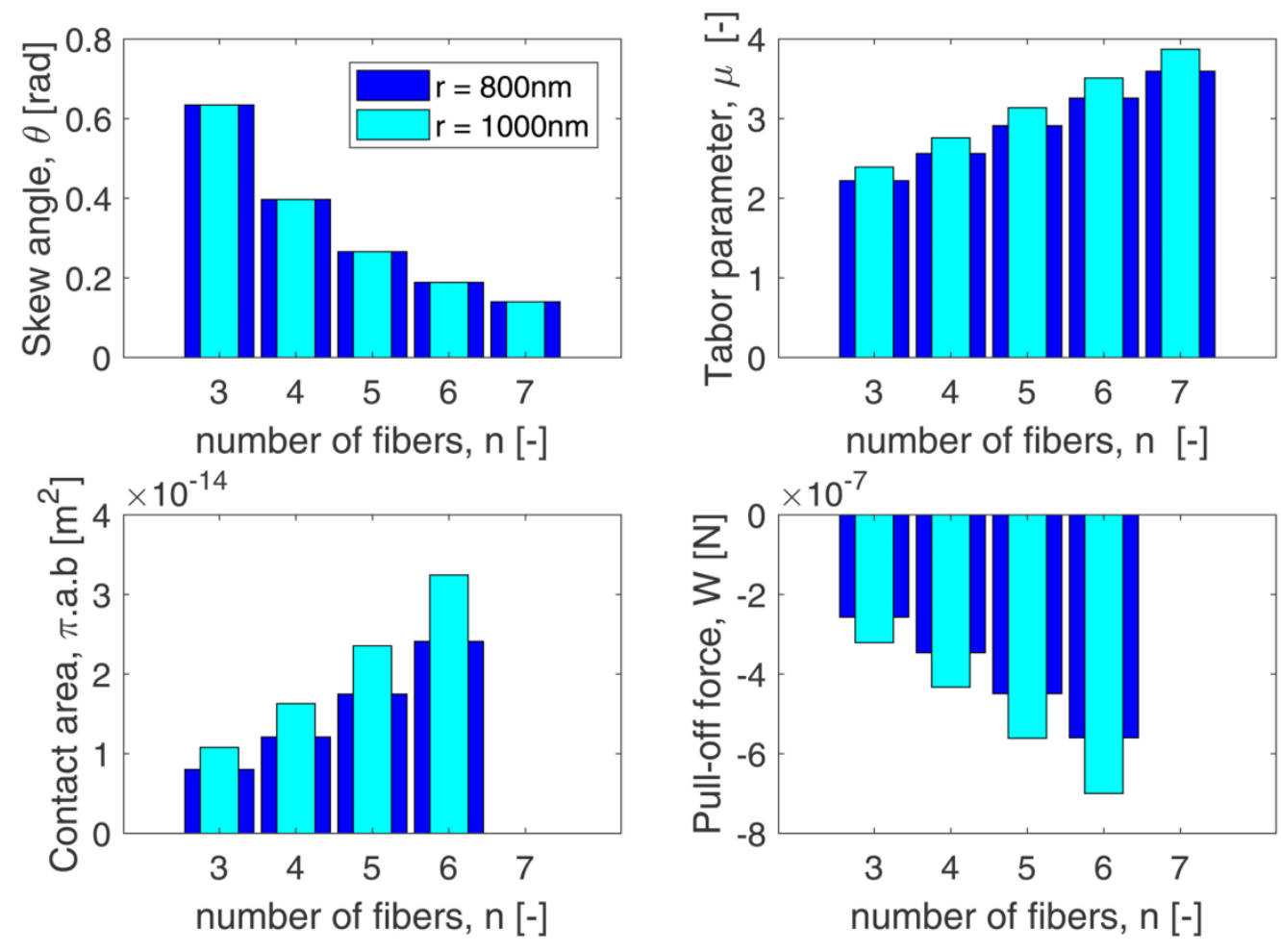

Fig. 3: The effect of the number of nanofibers in a nanoyarn and nanofiber radius on

(a) angle between the fibers, $\theta_{\text {skew, }}$ (b) Tabor parameter, $\mu$, (c) contact area, and (d) pull-off force, $W$.

\subsection{The effect of the nanofiber twisting speed $\left(\omega_{1}\right)$}

The effect of a higher nanofiber twisting speed $\omega_{1}$ of $5 \pi / 6$ is shown in Fig. 4. Higher $\omega_{1}$ is shown to result in higher $\theta_{\text {skew }}$, lower $\mu$ values, smaller contact area and lower pull-off force. Higher $\omega_{1}$ results in more nanofiber sections to be twisted, hence it increases the $\theta_{\text {skew }}$ between adjacent nanofibers. At $n=7$ for higher $\omega_{1}$ and at $n>3$ for higher $\omega_{2}$, the contact area and the pull-off force cannot be predicted as the $\theta_{\text {skew }}$ is too low for the JKR elliptical contact, similar to the situation at $n=7$ of previous results in Fig. 3 . 


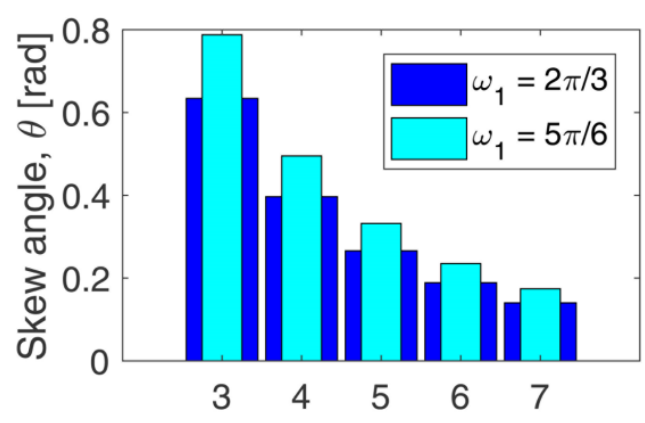

number of fibers, $\mathrm{n}[-]$

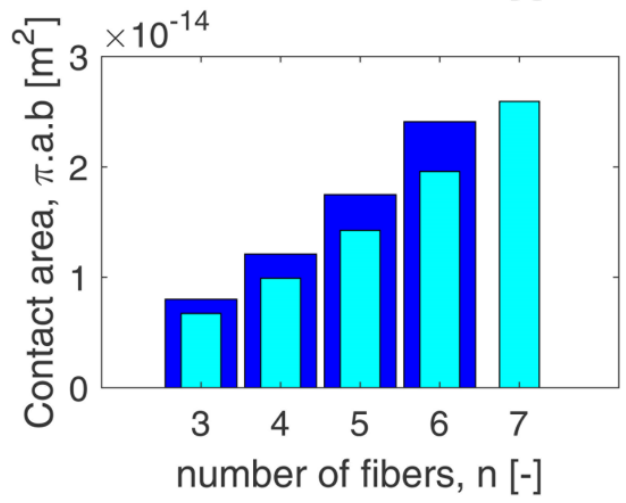

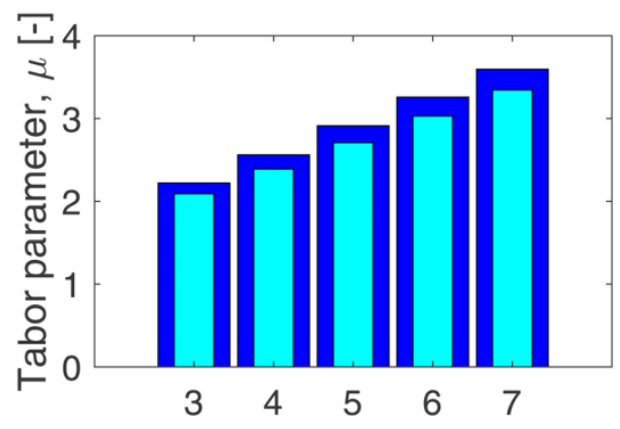

number of fibers, $\mathrm{n}[-]$

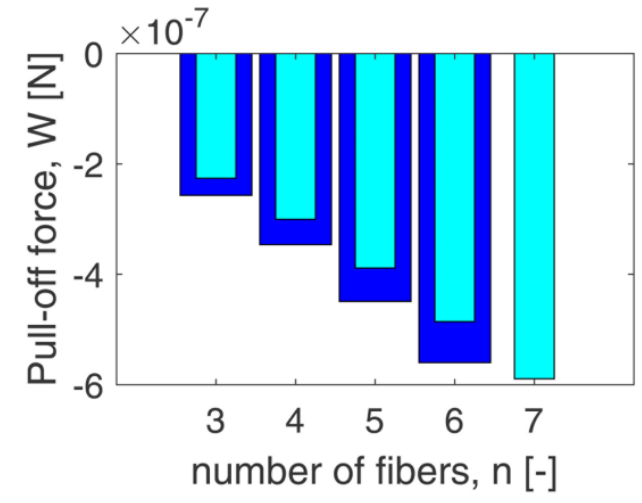

Fig. 4: The effect of nanofiber twisting speed on (a) angle between the fibers, $\theta_{\text {skew }}$ (b) Tabor parameter, $\mu$, (c) contact area, and (d) pull-off force, $W$.

\subsection{The effect of the nanoyarn reeling speed $\left(\omega_{2}\right)$}

The effect of nanoyarn reeling speed, $\omega_{2}$ is investigated next on adhesive nanofiber contacts in electrospun nanoyarns; higher $\omega_{2}$ of $\pi / 3$ is compared to the $\omega_{2}$ value from Table 2. It is shown in Fig. 5 that a higher $\omega_{2}$ results in lower $\theta_{\text {skew, }}$, higher $\mu$, larger contact area and greater pull-off forces. The effect of higher $\omega_{2}$ on adhesive nanofiber contacts in electrospun nanoyarns are available only for $n=3$. For a higher number of nanofibers, the corresponding $\theta_{\text {skew }}$ values are lower than the limit of the JKR elliptical contact, hence no adhesive prediction can be made. When results from both $\omega_{1}$ and $\omega_{2}$ are compared, the effect of $\omega_{2}$ is shown to be the opposite of $\omega_{1}$. 

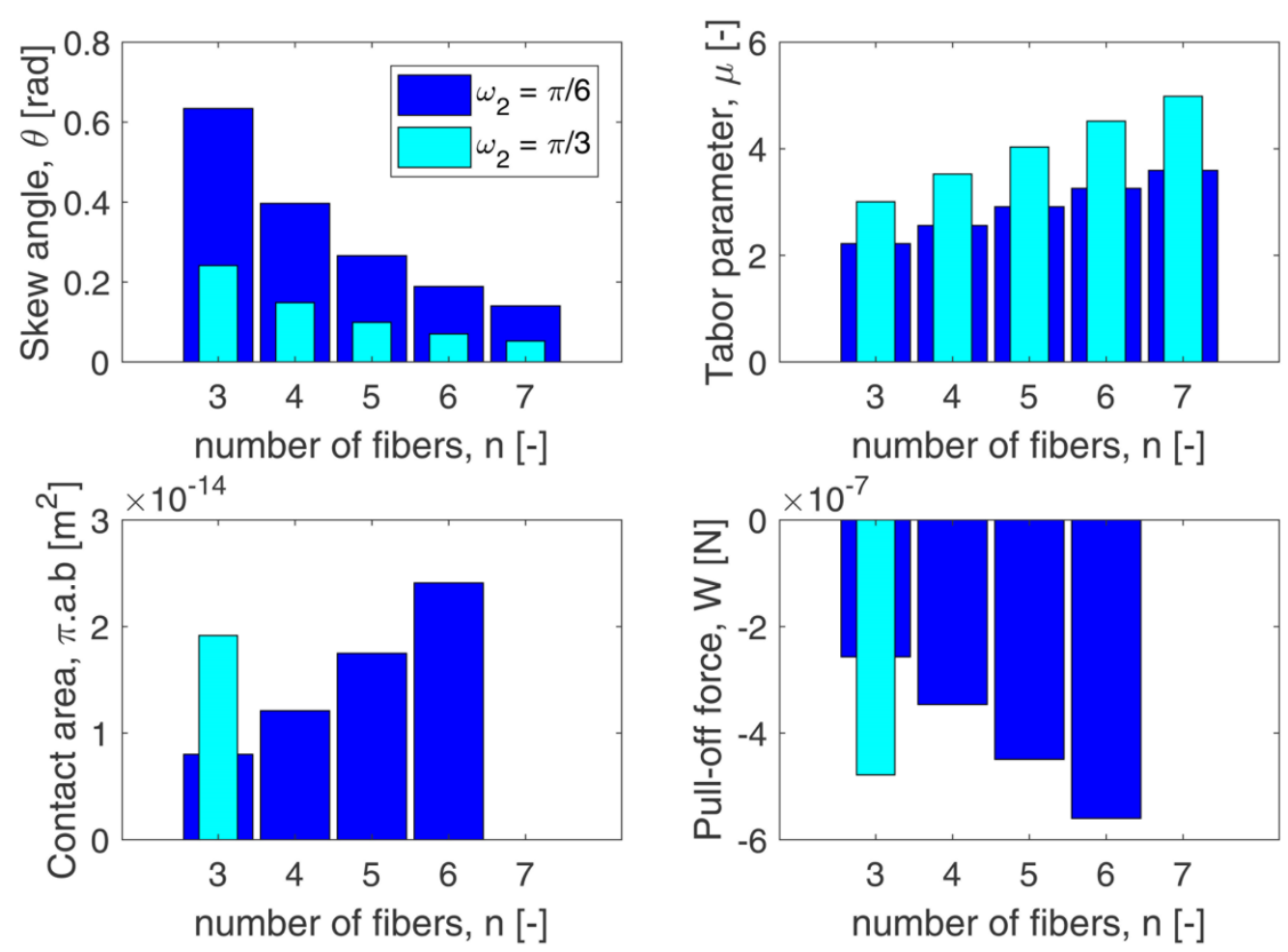

Fig. 5: The effect of nanoyarn reeling speed on (a) angle between the fibers, $\theta_{\text {skew, }}$ (b) Tabor parameter, $\mu$, (c) contact area, and (d) pull-off force, $W$.

\subsection{Significance of the presence of adhesion in fiber contacts within hierarchical structures}

Obviously, the adhesive force can be ignored at high load, as the adhesive load is smaller than the applied load. In this case, the contact can be regarded as a non-adhesive contact. Following the limit set in the adhesion map for circular contacts in [32] as a way of differentiating between adhesive contacts and non-adhesive contacts, the significance of the presence of adhesion between nanofibers in electrospun nanoyarns is determined using the ratio of the pull-off force to the total load, given as

$$
\left|\frac{W_{J K R}}{\text { Wtotal }}\right|<0.05
$$

Setting the maximum limit to 0.05 , it means that the contact is regarded as adhesive when the pull-off force is more than five percent of the total load. $W_{\text {total }}$ is given as:

$$
W_{\text {total }}=W_{J K R}+W_{f}
$$


Fig. 6 shows the result of the ratio between $W_{J K R} / W_{\text {total }}$ for all the parameters that have been analysed previously. The majority of the contacts have ratios higher than 0.05 , indicating that adhesion is indeed significant in those contacts. However, as the number of nanofibers increases, the nanoyarn becomes packed; the nanofibers are almost parallel to each other and have really low $\theta_{\text {skew. }}$. Hence, the JKR model cannot predict accurately the adhesive behaviour of these contacts.

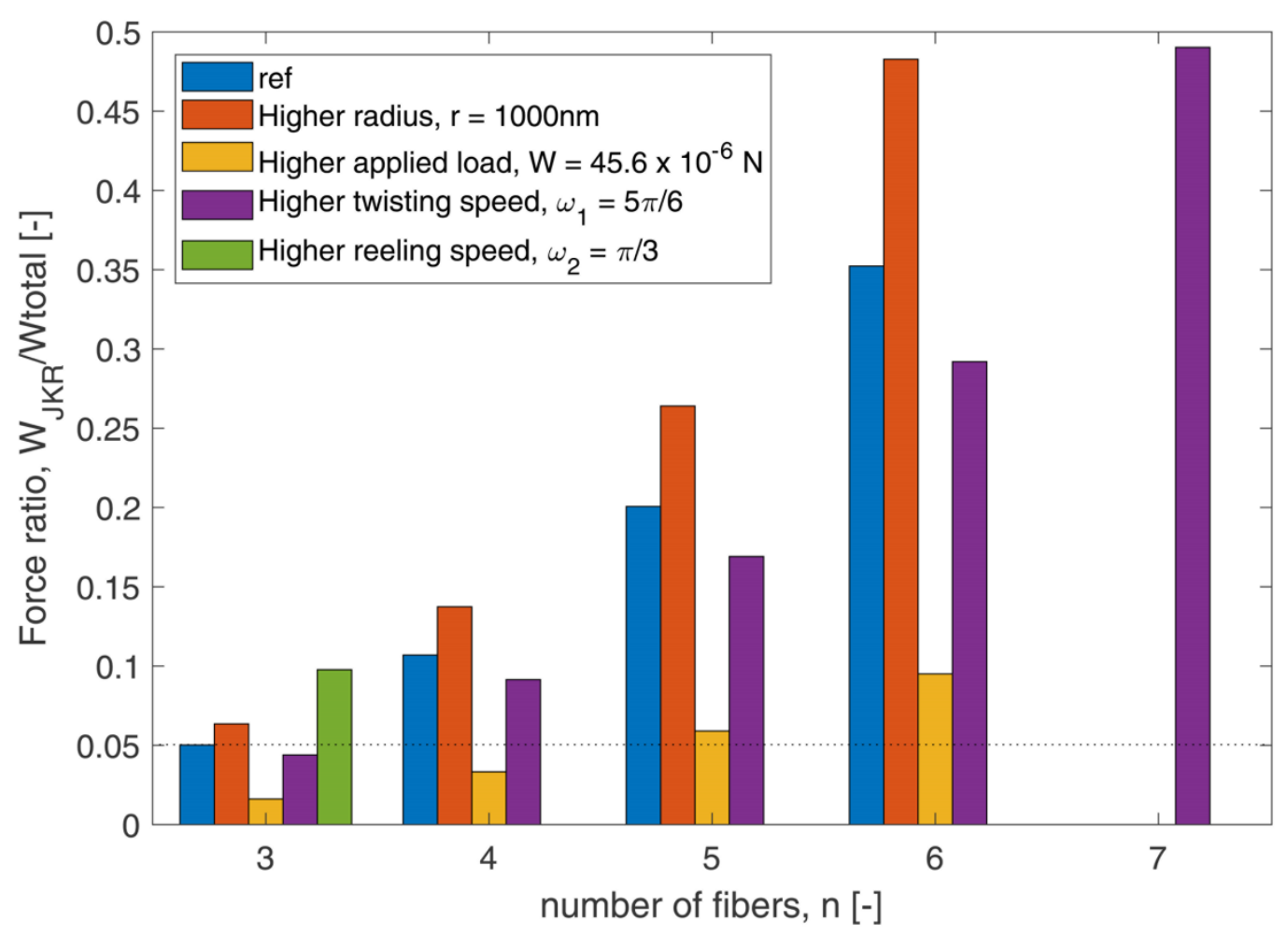

Fig 6: The force ratio for all parameters.

\section{Discussion}

Results show that for a low-order hierarchical structure such as electrospun nanoyarns, the presence of adhesion is indeed very significant in the contact between nanofibers. Compared to microfibers, nanofibers have higher tendency to deflect when nanoyarns are disturbed from outside sources such as air flow. Adjacent fibers in the nanoyarn may deflect and stick to each other as they are easily bent. As a result, adhesion-induced fiber collapse varies the connectivity and topology of the fiber network, and can lead to nonlinear behaviour and even larger adhesion area in the nanoyarns [33]. Adhesioninduced fiber collapse can also reduce the superior properties of nanoyarns that are based on their unique fibrous geometries. Hence, it is important to acknowledge the significant of the presence of adhesion especially for nanofiber contacts in nanoyarns. 


\section{Conclusions}

The significance of the presence of adhesion between nanofibers in electrospun nanoyarns is investigated in this paper. Load and contact properties of the nanofiber contacts in electrospun nanoyarns are obtained using the SWHS geometrical model, which then become the input for the JKR elliptical model. Electrospinning properties such as nanofiber radius, the number of nanofibers in a nanoyarn, nanofiber twisting speed and nanoyarn reeling speed are shown to influence adhesion at the nanofiber level. It has been shown that, in most cases, adhesion between nanofibers in electrospun nanoyarns cannot be ignored.

\section{Acknowledgement}

The authors gratefully acknowledge support from the Ministry of Education Malaysia, Universiti Teknikal Malaysia Melaka and Green Tribology and Engine Performance (GTriboE) research group.

\section{Appendix A}

$$
\begin{gathered}
K_{\text {coef }}=\tan (90-180 / n) \\
R_{1}=\left(d S \sqrt{\left(K_{\text {coef }}^{2}+1\right) /\left(S^{2}-\left(\pi K_{\text {coef }} d\right)^{2}\right)}\right) / 2 \\
\alpha_{1}=\tan ^{-1}\left(S / 2 \pi R_{1}\right) \\
\beta=\tan ^{-1}\left(2 \pi R_{1} / S\right) \\
t=\beta / \omega_{2}
\end{gathered}
$$

\section{Appendix B}

$$
\begin{gathered}
e^{2} \boldsymbol{D}(e)=\boldsymbol{K}(e)-\boldsymbol{E}(e) \\
\boldsymbol{B}(e)=\boldsymbol{K}(e)-\boldsymbol{D}(e) \\
e^{2} \boldsymbol{C}(e)=\boldsymbol{D}(e)-\boldsymbol{B}(e) \\
e^{2}=1-(b / a)^{2}
\end{gathered}
$$




\section{References}

[1] R. Lakes, "Materials with structural hierarchy", Nature, vol. 361, no. 6412, pp. 511515, 1993.

[2] C. Leech, The modelling and analysis of the mechanics of ropes. Dordrecht, Netherlands: Springer, 2013.

[3] U. Ali, Y. Zhou, X. Wang and T. Lin, "Electrospinning of Continuous Nanofiber Bundles and Twisted Nanofiber Yarns", in Nanofibers-production, properties and functional applications, T. Lin, Ed. InTech, 2011, pp. 153-174.

[4] A. Hamed, N. Shehata and M. Elosairy, "Investigation of Conical Spinneret in Generating More Dense and Compact Electrospun Nanofibers", Polymers, vol. 10, no. 1, p. 12, 2017.

[5] S. Jin, B. Xin and Y. Zheng, "Preparation and Characterization of Polysulfone Amide Nanoyarns by the Dynamic Rotating Electrospinning Method". Textile Research Journal, 0(00), 1-11, 2017.

[6] K. Kendall, "Adhesion: Molecules and Mechanics", Science, vol. 263, no. 5154, pp. 1720-1725, 1994.

[7] K. L. Johnson, K. Kendall and A. D. Roberts, "Surface energy and the contact of elastic solids", Proceedings of the Royal Society of London. Series A, Mathematical and Physical Sciences, vol. 324, pp. 301-313, 1971.

[8] B. Derjaguin, V. Muller and Y. Toporov, "Effect of contact deformations on the adhesion of particles", Journal of Colloid and Interface Science, vol. 53, no. 2, pp. 314-326, 1975.

[9] D. Maugis, "Adhesion of spheres: The JKR-DMT transition using a dugdale model", Journal of Colloid and Interface Science, vol. 150, no. 1, pp. 243-269, 1992.

[10] J. A. Greenwood and K. L. Johnson, "An alternative to the Maugis model of adhesion between elastic spheres", Journal of Physics D: Applied Physics, vol. 31, no. 22, pp. 3279-3290, 1998.

[11] M. Barquins, "Adherence and Rolling Kinetics of a Rigid Cylinder in Contact with a Natural Rubber Surface", The Journal of Adhesion, vol. 26, no. 1, pp. 1-12, 1988.

[12] Baney and C. Hui, "A cohesive zone model for the adhesion of cylinders", Journal of Adhesion Science and Technology, vol. 11, no. 3, pp. 393-406, 1997.

[13] K. L. Johnson and J. A. Greenwood, "Maugis analysis of adhesive line contact", Journal of Physics D: Applied Physics, vol. 41, no. 19, pp. 199802$199802,2008$. 
[14] F. Jin, W. Zhang, S. Zhang and X. Guo, "Adhesion between elastic cylinders based on the double-Hertz model", International Journal of Solids and Structures, vol. 51, no. 14, pp. 2706-2712, 2014.

[15] X. Wu and Y. A. Dzenis, "Adhesive contact in filaments", Journal of Physics D: Applied Physics, vol. 40, no. 14, pp. 4276-4280, 2007.

[16] K. L. Johnson and J. A. Greenwood, "An approximate JKR theory for elliptical contacts", Journal of Physics D: Applied Physics, vol. 38, no. 7, pp. 1042-1046, 2005.

[17] N. H. M. Zini, M. B. de Rooij, M. Bazr Afshan Fadafan, N. Ismail and D. J. Schipper, "Extending the Double-Hertz Model to Allow Modeling of an Adhesive Elliptical Contact", Tribology Letters, vol. 66, no. 1, 2018.

[18] N. H. M. Zini, M. B. de Rooij, M. Bazr Afshan Fadafan, N. Ismail and D. J. Schipper, "Response to Dr Greenwood's Comments on "Extending the DoubleHertz Model to Allow Modeling of an Adhesive Elliptical Contact'"', Tribology Letters, vol. 66, no. 3, 2018.

[19] B. Bhushan, A. Peressadko and T. Kim, "Adhesion analysis of two-level hierarchical morphology in natural attachment systems for 'smart adhesion'", Journal of Adhesion Science and Technology, vol. 20, no. 13, pp. 14751491, 2006.

[20] T. Kim and B. Bhushan, "Adhesion analysis of multi-level hierarchical attachment system contacting with a rough surface", Journal of Adhesion Science and Technology, vol. 21, no. 1, pp. 1-20, 2007.

[21] T. Kim and B. Bhushan, "The adhesion model considering capillarity for gecko attachment system", Journal of The Royal Society Interface, vol. 5, no. 20, pp. 319327, 2008.

[22] Q. Shi, K. Wan, S. Wong, P. Chen and T. Blackledge, "Do Electrospun Polymer Fibers Stick?", Langmuir, vol. 26, no. 17, pp. 14188-14193, 2010.

[23] M. Xing, W. Zhong, X. Xu and D. Thomson, "Adhesion Force Studies of Nanofibers and Nanoparticles", Langmuir, vol. 26, no. 14, pp. 11809-11814, 2010.

[24] Q. Shi, S. Wong, W. Ye, J. Hou, J. Zhao and J. Yin, "Mechanism of Adhesion between Polymer Fibers at Nanoscale Contacts", Langmuir, vol. 28, no. 10, pp. 4663-4671, 2012.

[25] S. Wang, X. Li, S. Lei, J. Zhou and Y. Yang, "Research on torsional fretting wear behaviors and damage mechanisms of stranded-wire helical spring", Journal of Mechanical Science and Technology, vol. 25, no. 8, pp. 2137-2147, 2011. 
[26] X. Li, S. Wang and J. Zhou, "Analysis of elliptical Hertz contact of steel wires of stranded-wire helical spring", Journal of Mechanical Science and Technology, vol. 28, no. 7, pp. 2797-2806, 2014.

[27] S. Wang, S. Lei, J. Zhou and H. Xiao, "Mathematical model for determination of strand twist angle and diameter in stranded-wire helical springs", Journal of Mechanical Science and Technology, vol. 24, no. 6, pp. 1203-1210, 2010.

[28] D. Tabor, "Surface forces and surface interactions", Journal of Colloid and Interface Science, vol. 58, no. 1, pp. 2-13, 1977.

[29] M. Bazbouz and G. Stylios, "The tensile properties of electrospun nylon 6 single nanofibers", Journal of Polymer Science Part B: Polymer Physics, vol. 48, no. 15, pp. 1719-1731, 2010.

[30] W. Callister and D. Rethwisch, Fundamentals of material science and engineering: an integrated approach, 3rd ed. John Wiley \& Sons, Inc, 2008.

[31] T. A. Skotheim and J. Reynolds, Conjugated polymers: processing and applications, CRC Press, 2006.

[32] K. L. Johnson and J. A. Greenwood, "An Adhesion Map for the Contact of Elastic Spheres", Journal of Colloid and Interface Science, vol. 192, no. 2, pp. 326-333, 1997.

[33] X. Wu and Y. A. Dzenis, "Collapse analysis of nanofibers", Nanotechnology, vol. 18, pp. 1-6, 2007. 
